\title{
Exploring the Dimensions of Eco-feedback in the Wild
}

DOCTORAL THESIS

Filipe Magno Gouveia Quintal

INFORMATICS ENGINEERING - HUMAN COMPUTER INTERACTION 


\section{Exploring the Dimensions of Eco-feedback in the Wild}

DOCTORAL THESIS

Filipe Magno Gouveia Quintal

INFORMATICS ENGINEERING - HUMAN COMPUTER INTERACTION

SUPERVISOR

Duarte Nuno Jardim Nunes

CO-SUPERVISOR

Valentina Nisi 


\section{Acknowledgements}

Foremost I want to express my appreciation for my thesis advisor Dr. Nuno Jardim Nunes and co-advisor Dr. Valentina Nisi for all the support, advice and patience during the development of this thesis.

I would also like to thank the rest of my thesis committee: Prof. Enrico Costanza and Prof. Nuno Correia, for their time, insightful comments and pertinent questions.

My thanks also goes to my lab colleagues in the Madeira-ITI: Ana Lúcia Faria, Athanasios Vourvopoulos, Augusto Esteves, Clinton Jorge, Mary Barreto, Michelle Scott and Lucas Pereira, for their support during this phase of my study and for all the fun we had in the last years.

Last but not the least, I would like to my parents, brothers, sister, friends and girlfriend which were decisive in the finals stages of writing phase. 



\section{Published work}

The majority of the work presented throughout this this was the result of publications in several interactional conferences in the fields of Engineering, Human Computer Interaction and Energy. In this chapter we list the practical work of this thesis and how it relates with the published contributions.

\section{Conceptual design of the eco-feedback (section 4.3.1)}

- Quintal, F., N. Nunes, A. Ocneanu, and M. Berges, "SINAIS: home consumption package: a low-cost eco-feedback energy-monitoring research platform", DIS '10: Proceedings of the 8th ACM Conference on Designing Interactive Systems, New York, NY, USA, ACM, pp. 419421,2010

\section{SINAIS Power Meter 1.0 (section 4.3.2)}

- Nunes, N., L. Pereira, F. Quintal, and M. Berges, "Deploying and evaluating the effectiveness of energy eco-feedback through a low-cost NILM solution", International Conference on Persuasive Technology (Persuasive '11), Columbus, OH, USA, 2011

\section{SINAIS Power Meter 2.0 (section 4.3.3)}

- Quintal, F., L. Pereira, and N. Nunes, "A long-term study of energy eco-feedback using nonintrusive load monitoring", International Conference on Persuasive Technology (Persuasive '12), [Poster], pp. 49, 06/2012.

- Pereira, L., F. Quintal, N. Nunes, and M. Bergés, "The design of a hardware-software platform for long-term energy eco-feedback research", 4th ACM SIGCHI symposium on Engineering interactive computing systems (EICS '12)

- Pereira, L., F. Quintal, M. Barreto, and N. Nunes, "Understanding the Limitations of Ecofeedback: a One Year Long-term Study", International Conference on Human Factors in Computing \& Informatics (SouthCHI '13) 
Shift in the feedback paradigm, mapping the consumption to the local landscape: SinaisFromFanal (section 4.4)

- Nisi, V., N. Nunes, F. Quintal, and M. Barreto, "SINAIS from Fanal: design and evaluation of an art-inspired eco-feedback system", Proceedings of the Biannual Conference of the Italian Chapter of SIGCHI.

\section{A nature-inspired portable eco-feedback device: WattsBurning (section 4.5)}

- Quintal, F., V. Nisi, N. Nunes, M. Barreto, and L. Pereira, "HomeTree - An Art Inspired Mobile Eco-feedback Visualization", Advances in Computer Entertainment Conference (ACE '12), [Demo]

- Quintal, F., L. Pereira, N. Nunes, V. Nisi, and M. Barreto, "WATTSBurning: design and evaluation of an innovative eco-feedback system", IFIP TC13 Conference on HumanComputer Interaction (INTERACT '13)

\section{Social enabled tangible eco-feedback: WattsBurning in my mailbox (section4.6)}

- Quintal, F., M. Barreto, N. Nunes, V. Nisi, and L. Pereira, "WattsBurning on my mailbox: a tangible art inspired eco-feedback visualization for sharing energy consumption", IFIP TC13 Conference on Human-Computer Interaction (INTERACT '13)

\section{Connecting electricity consumption with electricity production, the What-a-Watt system (section 4.7)}

- Quintal, F., L. Pereira, N. Nunes, and V. Nisi, "What-a-Watt : Where does my electricity comes from?", International Working Conference on Advanced Visual Interfaces (AVI '14) [Demo] 
- Quintal, F., L. Pereira, N. Nunes, and V. Nisi, "What-a-Watt: Exploring Electricity Production Literacy Through a Long Term Eco-Feedback Study", IFIP Conference on Sustainable Internet and ICT for Sustainability (SustainIT '15) [short paper], Madrid, Spain, IEEE Explore, 04/2015

\section{Watt-I-See: An interactive installation to probe eco-feed- forward strategies (section 4.8)}

- Quintal, F., C. Jorge, V. Nisi, N. Nunes, and M. Mendes, "Watt-a-Feeling - Raising Energy Production Literacy Through a Tangible Installation", AVI 2014 International Working Conference on Advanced Visual Interfaces [FSEA Workshop]

- Quintal, F., L. Pereira, C. Jorge, and N. Nunes, "EnerSpectrum: Exposing the source of energy through plug-level eco-feedack", IFIP Conference on Sustainable Internet and ICT for Sustainability (SustainIT '15) [poster abstract]

\section{Other publications}

- Esteves, A., F. Quintal, and I. Oakley, "TouchCloud: Enabling People to Augment Real-world Objects With Cloud-stored Data", Human Computer Interaction Korea (HCI Korea '14), 2014

- Barreto, M., A. Szóstek, E. Karapanos, N. Nunes, L. Pereira, and F. Quintal, "Understanding families' motivations for sustainable behaviors", Computers in Human Behavior, vol. 40, pp. 6 $-15,11 / 2014$

- Pereira, L., F. Quintal, R. Gonçalves, and N. Nunes, "SustData: A Public Dataset for ICT4S Electric Energy Research", International Conference on ICT for Sustainability (ICT4S '14)

- Esteves, A., F. Quintal, and I. Oakley, "TouchCloud: An Exploratory Study in Physically Tagging the Cloud", International Conference on Tangible, Embedded and Embodied Interaction, 8th: ACM, 2014

- Esteves, A., F. Quintal, and I. Oakley, "jamTable: Can Physical Interfaces Support the Collaboration Between Novice and Experienced Musicians?", 8th International Workshop on Haptic and Audio Interaction Design : Springer, 2013

- Scott, M., M. Barreto, F. Quintal, and I. Oakley, "Understanding Goal Setting Behavior in the Context of Energy Consumption Reduction", Human-Computer Interaction - INTERACT 2011

- Tiago, C., F. Quintal, M. Scott, V. Kostakos, and I. Oakley, Towards Egocentric Fuel Efficiency Feedback, : CHI 2011, PNIC workshop, 2010 



\section{Abstract}

Worldwide reports about energy usage have noted the importance of the domestic energy consumption sector in the worldwide scenario. This fact motivated and supported the birth and spread of the so-called eco-feedback devices. Such devices provide information about individual or group energy consumption behavior with the goal of reducing the impact in the environment. Motivated by the body of work which questions the long term effectiveness of eco-feedback systems, this thesis focus on evaluating in-the-wild the long term usage of eco-feedback systems. We have conducted five long term studies with different eco-feedback systems designed to evaluate different dimensions in the design of eco-feedback plus two more focused short term studies aimed at studying concrete approaches.

Our summary reports on the fact that the there is a novelty effect associated with ecofeedback systems in which the usage of these devices declines significantly after a month. We did not found evidence that the novelty effect is related to location or the type of information represented, nor that the decrease in the eco-feedback usage could lead to the consumption relapsing to values previous to the introduction of the eco-feedback

Our work has also generated other contributions related to the positioning of the feedback, using metaphors for representing the consumption and presenting information about the source of the energy in the feedback. 



\section{Table of contents}

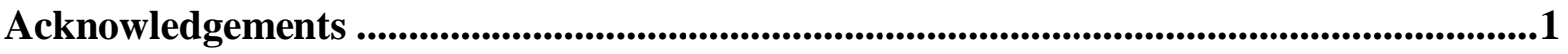

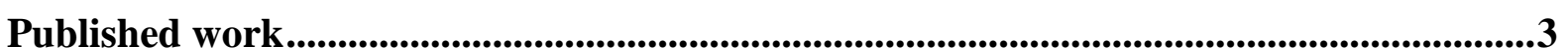

Conceptual design of the eco-feedback (section 4.3.1) ...................................................................3

SINAIS Power Meter 1.0 (section 4.3.2) ..................................................................................3

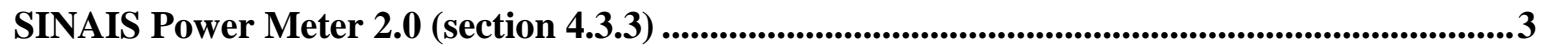

Shift in the feedback paradigm, mapping the consumption to the local landscape:

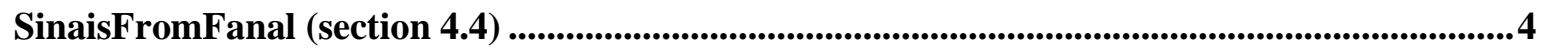

A nature-inspired portable eco-feedback device: WattsBurning (section 4.5) ...............................4

Social enabled tangible eco-feedback: WattsBurning in my mailbox (section4.6) .......................4

Connecting electricity consumption with electricity production, the What-a-Watt system

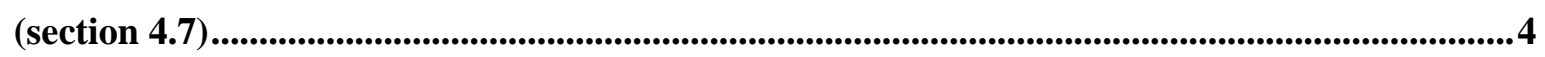

Watt-I-See: An interactive installation to probe eco-feed-forward strategies (section 4.8) ........5

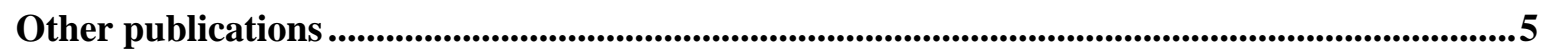

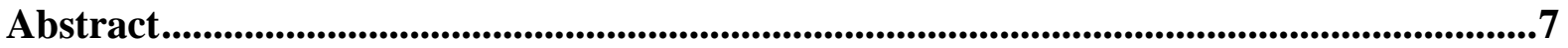

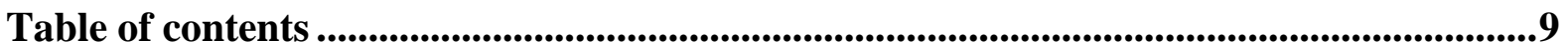

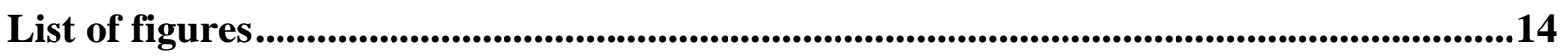

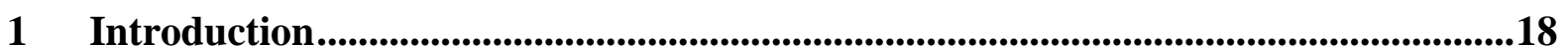

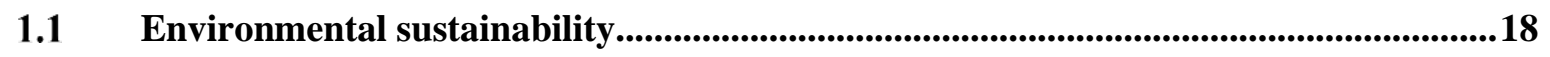

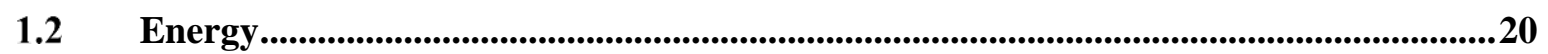

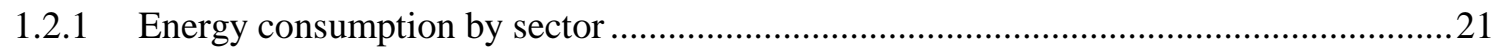

1.3 Domestic energy consumption...............................................................................................22

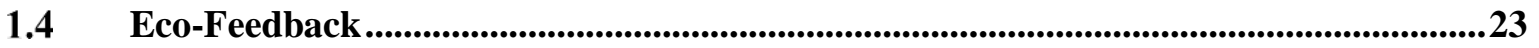

1.5 The SINAIS Research Project ..................................................................................................24

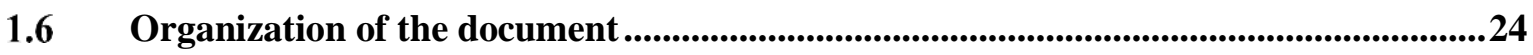

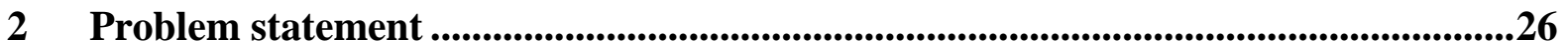

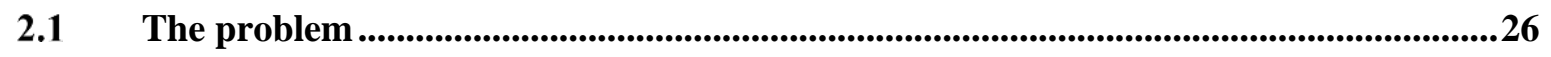

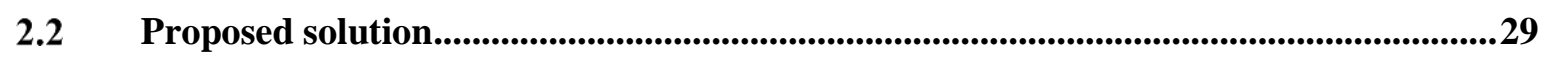

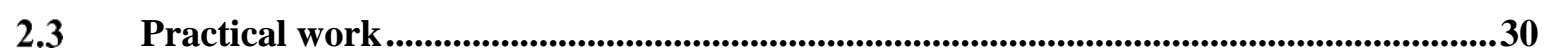

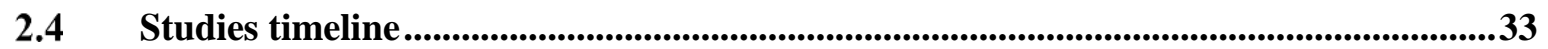


3 Background: A Survey of Energy Monitoring and Eco-Feedback Technologies....36

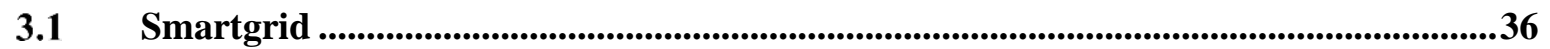

$3.2 \quad$ Sensing Technologies...........................................................................................................38

3.3 Design of eco-feedback systems ............................................................................................41

$3.4 \quad$ Eco-feedback display medium ............................................................................................44

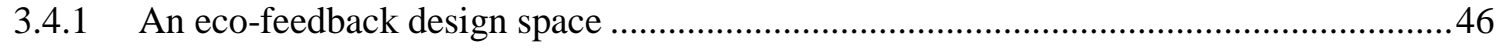

3.5 Strategies to reduce consumption ....................................................................................................49

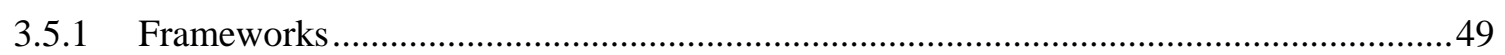

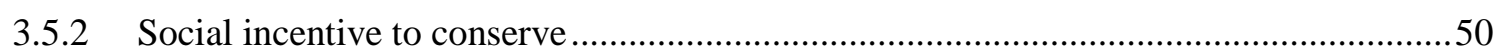

3.5.3 Displaying the impacts of energy consumption in the environment ...............................54

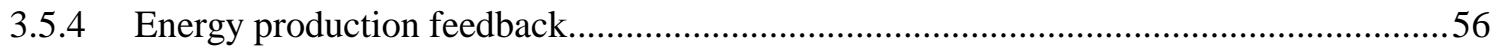

3.6 Effectiveness of eco-feedback over time ....................................................................................59

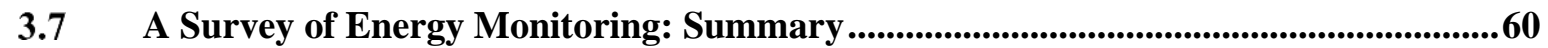

$4 \quad$ Research Studies ..........................................................................................................................71

$4.1 \quad$ Deployments Setting ...........................................................................................................71

4.2 Research Hardware Platform.......................................................................................................74

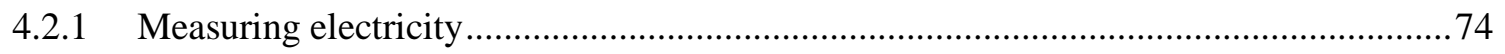

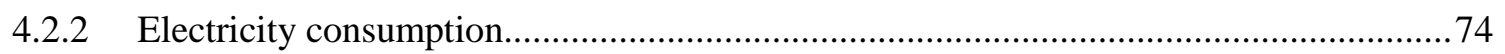

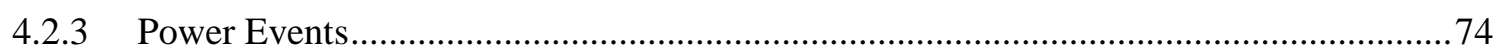

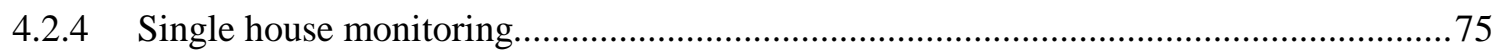

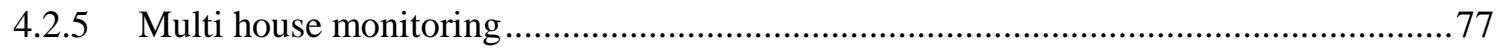

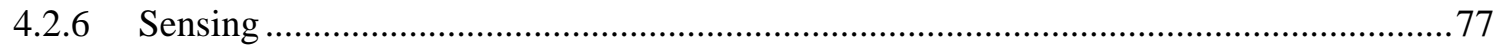

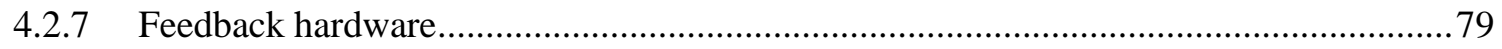

$4.3 \quad$ Background Work ..............................................................................................................................82

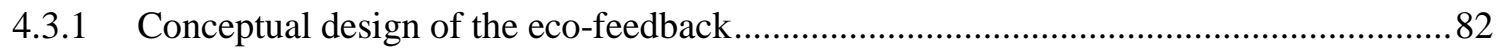

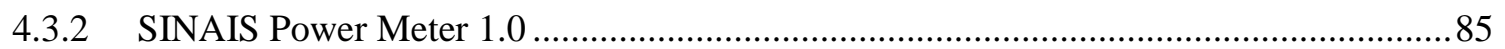

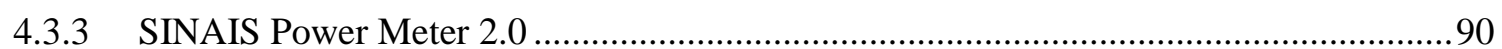

4.4 Shift in the feedback paradigm, mapping the consumption to the local landscape:

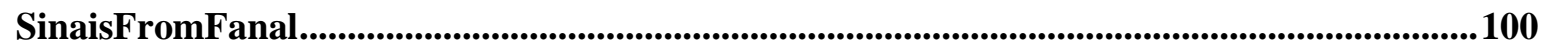

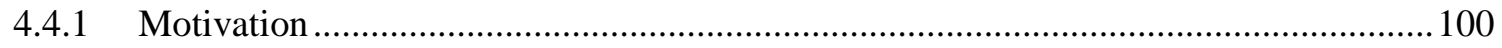

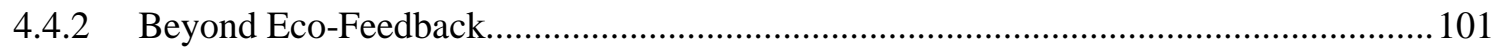

4.4.3 Visualization - Mapping electricity consumption to the local forest............................. 102

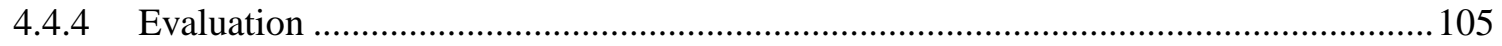

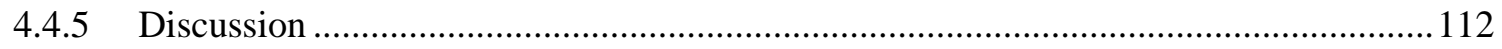

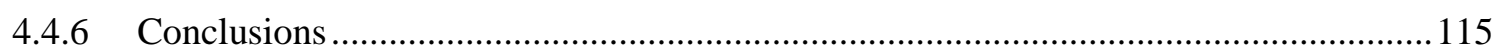

4.5 A nature-inspired portable eco-feedback device: WattsBurning.................................116 


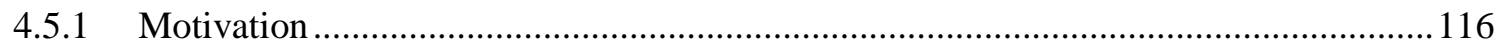

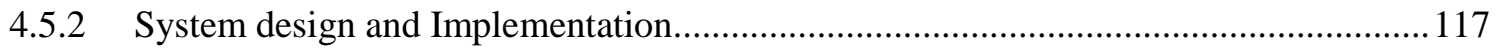

4.5.3 WattsBurning eco-feedback visualization...................................................................... 117

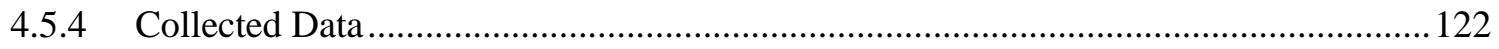

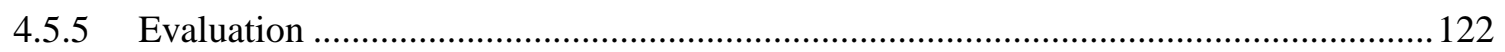

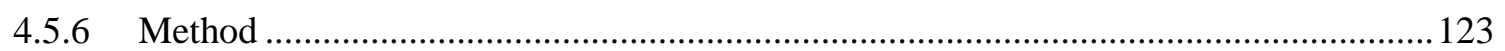

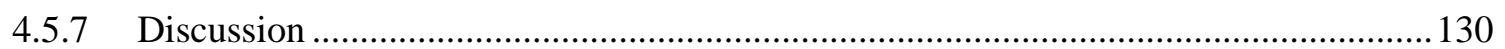

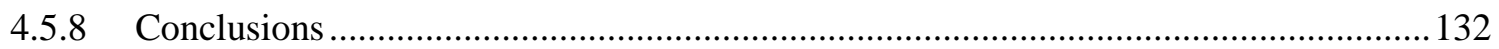

4.6 Social enabled tangible eco-feedback: WattsBurning in my mailbox............................134

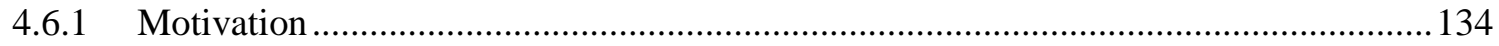

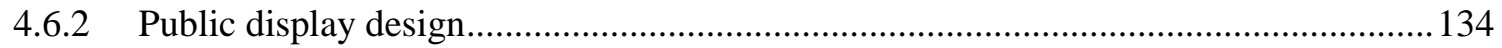

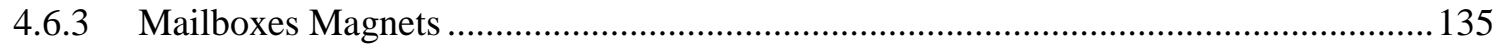

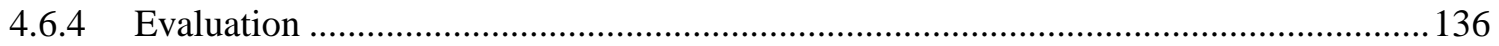

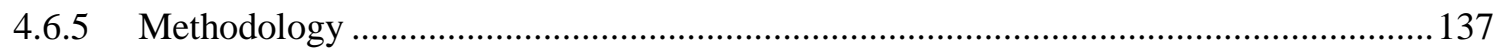

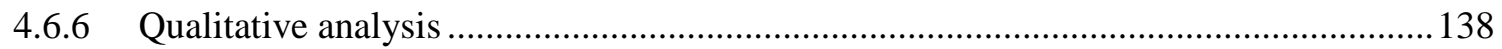

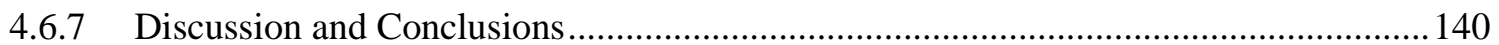

\subsection{Connecting electricity consumption with electricity production, the What-a-Watt} system 142

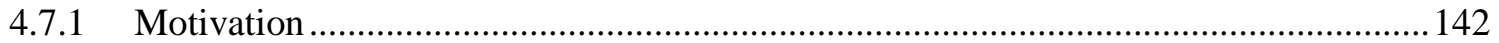

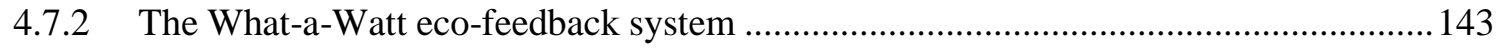

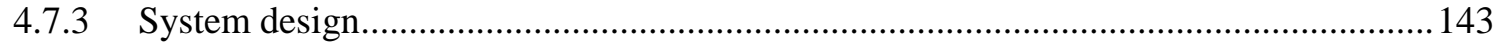

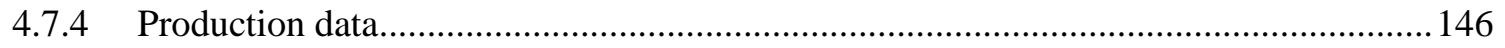

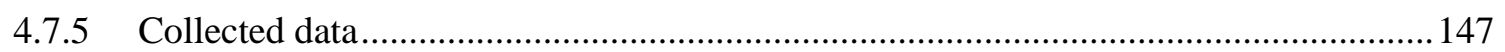

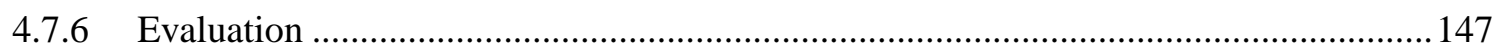

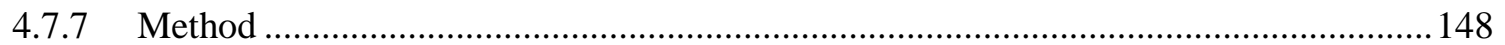

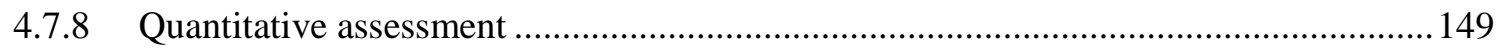

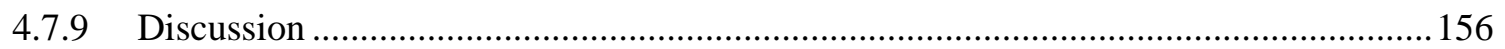

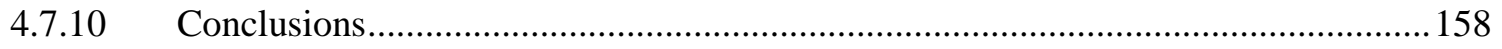

4.8 Watt-I-See: An interactive installation to probe eco-feed-forward strategies ............160

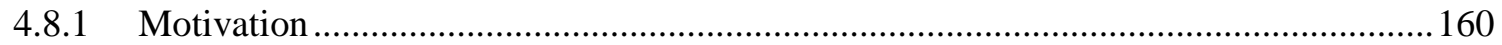

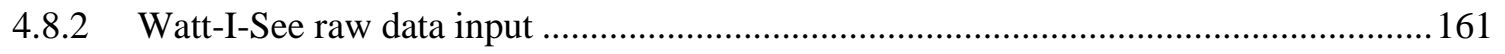

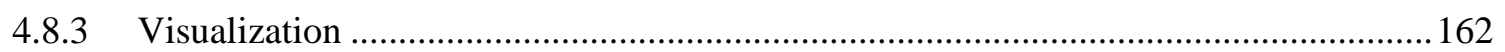

4.8.4 The Glowing Power Socket: Renewable Energy Feedback ........................................ 163

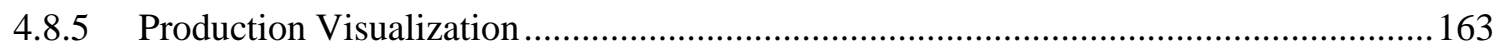

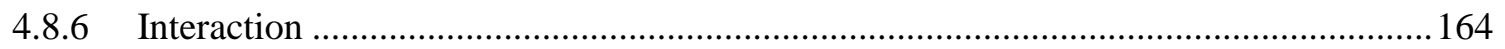

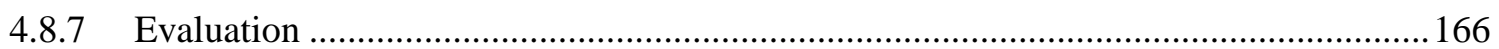




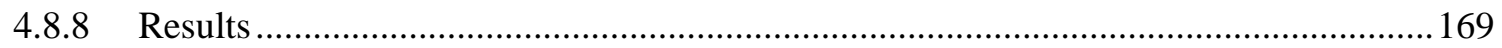

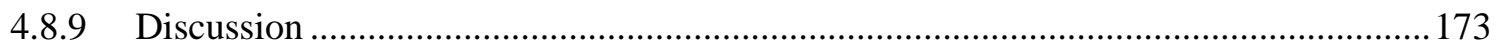

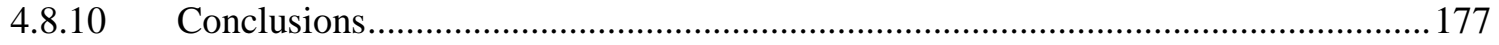

5 Summary of research studies.........................................................................................180

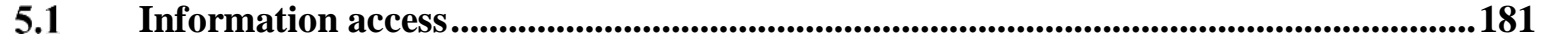

$5.2 \quad$ Interactivity ..........................................................................................................................181

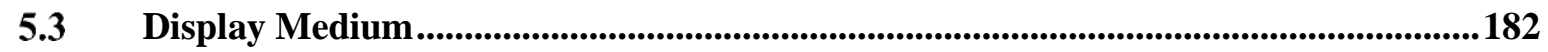

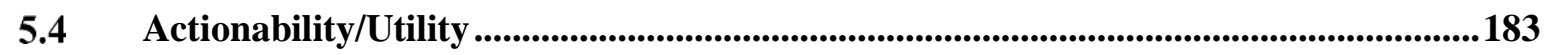

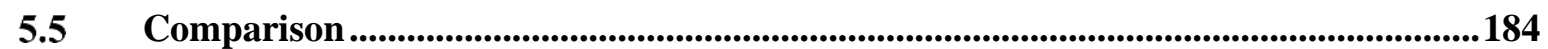

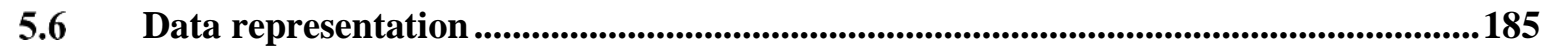

$5.7 \quad$ Social Aspects.............................................................................................................................186

5.8 Motivation/Persuasive techniques........................................................................................187

5.9 Comparison Aspects ......................................................................................................................187

6 Conclusions ......................................................................................................................190

6.1 RQ1: Can we detect the novelty effect in eco-feedback systems deployed in the wild? 190

6.1.1 Implications.

6.2 RQ2: Is the novelty effect related to variables such as location, representation and information?

6.2.1 Implications.

6.3 RQ3:Can the novelty effect be related to relapsing behaviour prior to the exposure of the eco-feedback?................................................................................................................................................... 191

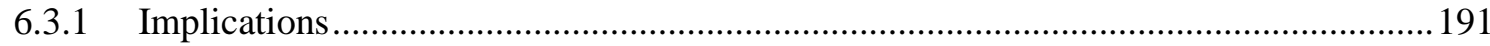

6.4 Other contributions ...............................................................................................................192

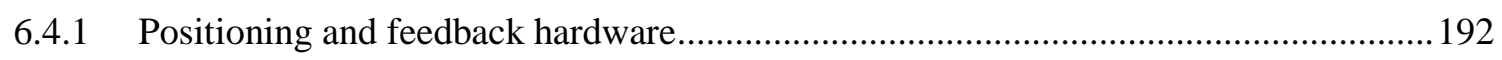

6.4.2 Using metaphors for displaying energy consumption ................................................. 192

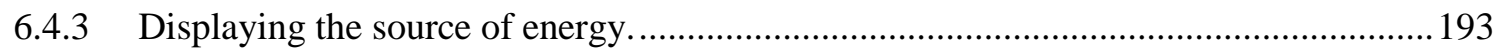

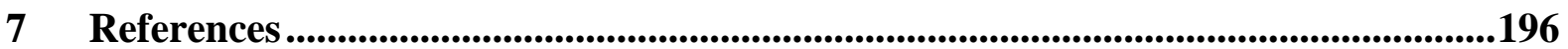

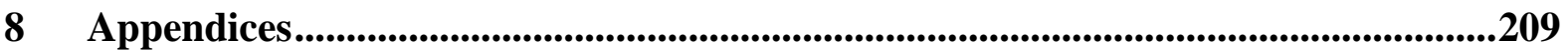

8.1 Selection survey Sinais Power Meter 1.0 ...........................................................................209

8.2 Participants Survey Sinais Power Meter 1.0 ....................................................................2213

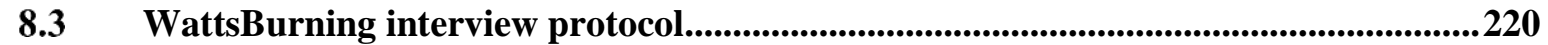

8.4 WattsBurning in my mailbox interview protocol ..............................................................222

8.6 What-a-Watt pre interview questionnaire ................................................................................223

8.7 What-a-Watt interview protocol ..........................................................................................226 
8.8 What-I-See pre study questionnaire .....................................................................................228

8.9 What-I-See interview protocol ......................................................................................................2232

8.10 What-I-See post study questionnaire .........................................................................................2234

8.11 What-I-See EV2 questionnaire...............................................................................................236 



\section{List of figures}

Figure 1:Graphical representation for the different subsets of studies discussed in the problem statement.

Figure 2: Timeline which places our practical studies along the time period of this dissertation.

Figure 3: Example of a smart meter based on Open smart grid protocol (OSGP) in use in Europe that has the ability to reduce load, disconnect-reconnect remotely, and interface to gas and water meters (EVB Energy Ltd, 2008).

Figure 4: Left: HydroSense sensor system (J. E. Froehlich et al., 2009) Right: Electrisense system (Gupta et al., 2010). .41

Figure 5: Left: Abstract representation for water savings from (Froehlich et al., 2012); Middle: Meter used in (Yun, 2009); Right: Screenshot of the mobile application used in (Gamberini et al., 2012a). ........44

Figure 6:: Different models of commercial energy monitors, Wattson, Current Cost and Kill a Watt......46

Figure 7: Left; Public display used in (Moere et al., 2011); Middle: StepGreen Social network (Mankoff et al., 2010b); Right: Projections used in the WattLite prototype (Jönsson et al., 2010). .54

Figure 8: Left: 7000 oaks and counting system (Holmes, 2007a); Middle: Erratic radio developed by the Static! Institute (Ernevi et al., 2009). Right: Ubigreen mobile application (J. Froehlich et al., 2009).

Figure 9: Left: The local energy indicator (Pierce and Paulos, 2012); Right: Visualization used in (Filonik et al., 2013).

Figure 10: Left: City of Funchal, the capital of Madeira. Right: Madeira endemic laurissilva forest........72

Figure 11: Time line of the different electricity components in a household, the power events are signed by red rectangles. . .75

Figure 12: Current clamp and transformer used to measure current and voltage. 76

Figure 13: The system as it was deployed in one of the buildings of the WattsBurning study. On the left is the DAQ Board and the voltage transformers. On the right is the current clamp sensing each house.

Figure 14: Overall architecture of the hardware + software platform. 79

Figure 15: Android tablet used as a feedback client in the second version of our hardware. This particular tablet is running the What-a-Watt eco-feedback system. 80 
Figure 16: Electricity meter used in the pilot. Left: Efergy system; Middle: Current Cost eco-feedback system; Right: Owl electricity monitoring system

Figure 17: Notebook installed at the main fuse box of the household. This configuration was used in the first version of our hardware.

Figure 18: Top Left: Attract mode of the system, Top Right and Bottom: Different options presented in the Active Mode of the prototype. 84

Figure 19: Different views of the SINAIS Power Meter 1.0: top left: spectrum graphic with the consumption of the last 8 hours; top right: consumption throughout the week and comparison with previous week; bottom left: daily consumption; bottom right comparison between different days of the month......86

Figure 20: Interactions with the SINAIS Power Meter 1.0 system. The grey bars represent the sum of all interaction and the red line represents the intentional interactions.

Figure 21: Second version of the eco-feedback system. Top right: Daily consumption; Top left: Monthly consumption; Bottom left: View of the system when no movement is detected near the meter after 2 minutes; Bottom right: User interacting with the system. .91

Figure 22: Madeira Laurisilva landscape. 101

Figure 23: Screenshot of the application used in the SinaisFromFanal study. On top there's the main view of the forest, the consumption is mapped as the movement of the clouds in the background. At the bottom there is the landscape with elements added based on the usage of appliances. .105

Figure 24: Different levels of engagement of users with the SinaisFromFanal eco-feedback system and corresponding response. 105

Figure 25: Sum of the weekly interaction with system before (red) and after the SinaisFromFanal study, the dotted line represents the date of family interviews. .107

Figure 26: Hardware used for the WattsBurning eco-feedback system. 118

Figure 27: Different landscape visualizations developed by the involved artists and used during the thinkaloud process to include the users in the choice of their favourite landscape.

Figure 28: Different views of the landscape according to the consumption. Ranging from low consumption (Image 1) to high consumption (image 5).

Figure 29: Left: Home screen of the system. Right: Tab with the consumption of the current week. .122

Figure 30: Consumption in the 6 households during the study. The baseline period is highlighted in red. The horizontal axis represents the week in the study, and the vertical represents the average consumption in $\mathrm{kWh}$. 127

Figure 31: Interaction with the WattsBurning system during the fifteen weeks of the deployment. 130 
Figure 32: The 3 different stages of the forest represented by the magnets (banner is on top). 1. Banner with the week days. 2. Forest with low consumption. 3. Forest with medium consumption. 4. Forest with high consumption.

Figure 33: Mailbox of one participant at the beginning (left) and at the end of the study (right). 137

Figure 34: Image of the apartment complex used in the WattsBurning on my mailbox study. The yellow dots mark the positioning of the mailboxes in the selected buildings. .138

Figure 35: Different interface prototypes sketches developed during the design of the production view for the What-a-Watt system.

Figure 36: Interface screen displaying the island electricity production and the household consumption. In this particular moment there was $10 \%$ of renewable energy present in the grid, and the household was consuming less $22 \%$ than its average.

Figure 37: Left: Home view of the What-a-Watt system displaying averages and comparisons of consumed and produced energy between time periods. Right: Interface screen displaying the consumption throughout the week.

Figure 38: Time series with the average weekly consumption throughout the study in the three groups: Orange line - Study group; Green Line - Control group; Red line - Whole building. 151

Figure 39:WISE panel displaying low presence of renewable energy

Figure 40: Five possible stages of the Power Socket, from high presence of renewable energy (left), to low presence of renewable energy (right).

Figure 41: Left: Children observing the WISE's visualization; Right: Legos used in the interaction with children. .165

Figure 42: Card used to manipulate WISE by EV1 165

Figure 43: Fifth question of the children's evaluation of WISE.

Figure 44: Colors used to represent our systems in the classification according Froehlich's design space

Figure 45: Information access classification of the practical work of this thesis.

Figure 46: Interactivity classification of the practical work of this thesis.

Figure 47: Display medium classification of the practical work of this thesis.

Figure 48: Actionability/Utility classification of the practical work of this thesis.

Figure 49: Comparison classification of the practical work of this thesis. 
Figure 50: Data representation aspects of the practical work of this thesis. ..

Figure 51: Social Aspects classification of the practical work of this thesis. ..........................................186 


\section{Introduction}

In this chapter, we provide the introduction and background motivation of this thesis. Our work was firstly motivated by the impact of the energy sector, more precisely the domestic energy sub-sector and how it influences environmental degradation and impacts environmental sustainability. From there we focus in the core of our work, the design implementation and testing of devices that provide individuals information about their energy consumption, their effectiveness and how they are adopted by families/individuals.

In the following sections of this chapter we describe this thesis motivation in detail. Starting from an overview of environmental sustainability to the impact of the energy sector in the environment, and how we envisioned several concrete approaches to mitigate environmental degradation through eco-feedback technologies.

\subsection{Environmental sustainability}

Environmental sustainability is the set of practices that ensure the long-term quality of the environmental. These practices aim at avoiding the exhaustion of resources or poisoning of the environment phenomena normally associated with environmental degradation.

Environmental degradation is the process by which the environment is damaged or corrupted in some form (Johnson et al., 1997). Nowadays, environmental degradation is widely considered as one of the biggest threats facing our planet. This phenomenon is visible in the exhaustion or deterioration of resources such as water, air and soil, which could lead to the destruction of ecosystems. Environmental degradation can occur naturally, or it can be caused or accelerated by human interventions.

The exhaustion of resources is simply the depletion of any given resource, for instance, be it water, soil or air. This depletion can be caused by the human overuse of these resources, 
or by changes in the natural habitats causing unbalanced consumption of resources leading to migrations or even extinctions.

The effect of human activities on the environment and the resulting carbon emissions began to be studied in the 1960's. By the end of the 1980's scientists were able to draw a first sketch of how human activities were affecting the earth's temperature (Schipper et al., 1989). Currently, scientists have a better understanding of human-influenced global warming, and how it relates to air pollution, water quality/availability and environmental degradation. Human existence is proven to have different effects on the environment, in fact authors have claimed that there are no terrestrial environments that do not bear some evidence of human action (Tilman and Lehman, 2001).

The human impact on the environment has been described by the I=PAT formula (more precisely $\mathrm{I}=\mathrm{P} * \mathrm{~A} * \mathrm{~T}$ ) (Inc, 1972). This formula defines the impact on the environment (I), as a combination of $\mathrm{P}$ : the increase in population of an area (which can lead to an increase in resource demand as well as an increase in pollution); A: the affluence of the population (which consists of the average energy consumed by each individual in the population); and T: which stands for technology (relating to the quantity of resources consumed to produce the affluence described above). This formula provides a very general definition of human impact on the environment, and for this reason it has been the target of critics (Alcott, 2010), that claim that the formula is too simple to define a complex problem such as the impact of humans on the environment. They claim that the impact should be defined as a function of Population, Affluence and Technology, three aspects which are not independent of each other, and that the I=PAT formula does not take observation into account.

The fact is that most of the actions and habits common in different societes have the potential to be damaging for the environment. Different agricultural techniques used around the world can cause deforestation, deviation of watercourses, or soil exhaustion. Overfishing can lead to ecosystem destruction resulting in environmental degradation. The industrial process of assembly and production also contributes to degradation caused by deforestation (e.g. the paper industry), and accounts for noxious emissions to the environment. Mining and logging are, by definition, the gathering of resources from nature, and can lead to ecosystem loss, soil erosion/poisoning. Modern means of transportation are also significantly polluting in the worldwide environment, since they mostly use a sub-product of petroleum as fuel, resulting in $\mathrm{CO}_{2}$ emissions in the atmosphere (in fact, road transport is by subsector the largest contributor to $\mathrm{CO}_{2}$ emissions). Furthermore, the construction of transportation routes (for examples railways, or highways) can result in deforestation. Lastly the energy industry, is responsible for 
environmental degradation, as the worldwide demand for energy rises (International Energy Outlook 2013, 2013). In the next section we present in detail the impact that the different energy sub-sectors have globally.

\subsection{Energy}

In this section we refer to energy in general terms as "power derived from the utilization of physical or chemical resources, especially to provide light and heat or to work machines". Energy is "the" commodity of modern developed societies and therefore impacts worldwide economies and industry.

Petroleum is probably the most commonly known raw material used in the energy sector. Its use (e.g. burning fuel), extraction (e.g. hydraulic fracturing) and raw form are all considered harmful to the environment. As a consequence, petroleum is commonly considered the main cause for environmental degradation. However, alternative forms of energy such as nuclear or coal also create significant negative effects on the environment, since they can produce noxious emissions and/or rely on mining which causes environmental degradation.

Alongside the fuel that powers our cars, electricity and the electrical grid are the most common means of providing energy to individuals, as it is used in houses, cities and public services. The production and distribution of electricity as it stands nowadays contributes to environmental degradation since it uses harvested elements from the environment (coal, petroleum, sun, wind, etc.). However not all production techniques impact the environment equally. Thermal stations burn fossil fuels (petroleum, coal, etc.) to generate electricity. This process generates $\mathrm{CO}_{2}$ and, depending on the fuel and particular techniques used, it can also emit other harmful elements to the environment, such as methane or nitrous oxide. Nuclear plants do not produce $\mathrm{CO}^{2}$ emissions during their normal operation. However, the mining, preparation and transportation of the elements used in nuclear processes all emit carbon, yet these values are quite small when compared to the long-term emissions of thermal fuel plants. Nonetheless, the noxious radiations emitted by nuclear stations are strictly controlled by regulations, and situations in which these regulations have not been met are scarce, although the impact of any isolated occurrences are long-lasting and have a strong negative impact on the ecosystem (Alexakhin et al., 2006). Biomass stations work in a similar manner to thermal stations that burn fossil fuels. However biomass stations burn other materials that can be combusted (for example wood), or use the natural decomposition of biological composites that 
produces gas (commonly called biogas), which is then burned to produce electricity. The process of using biomass to generate electricity emits approximately the same quantity of $\mathrm{CO}_{2}$ as the thermal fuel station. However this is considered a carbon neutral process since the emitted $\mathrm{CO}_{2}$ had previously been absorbed from the atmosphere by the growing biomass. The indirect impact of biomass electricity generation is related to the deforestation and soil erosion that is normally associated with agricultural techniques used when growing the biomass.

Other techniques used for electricity generation such as wind/tidal farms, hydropower, geothermal power harvesting, solar power and solar thermal power have significantly lower emissions (in some cases zero emissions when operating optimally). Therefore, their impact on the environment is related to the resources (materials, land and energy) used in construction and operating the equipment used to harvest the energy (e.g. windmills, photovoltaic panels).

Data from 2010 reveals that the main raw resource used to produce electricity worldwide (actual quotas differ from country to country) is still coal (40\% of the total) followed by natural gas $(22 \%)$, the sum of all renewables (20\%), nuclear (12\%) and oil (4\%). Projections for 2015 estimates that the demand for energy from all sources, except oil, is due to increase. Petroleum, however, is predicted to remain around the same values of demand. Considering the renewable electricity generation techniques, which accounts for $20 \%$ of total electricity produced worldwide, hydroelectric accounts for $80 \%$, wind for $8 \%$, geothermal generation techniques account for $1.5 \%$, solar for $0.8 \%$, and other assorted techniques account for $8 \%$ (International Energy Outlook 2013, 2013).

\subsubsection{Energy consumption by sector}

Energy consumption worldwide is divided among different sectors: industry, transportation, building, residential and commercial sectors. The industry sector includes the energy used in manufacturing, building or harvesting goods used in our society (for example in agriculture or construction). This is the largest sector energy-consumption-wise, responsible for $53 \%$ of worldwide energy consumption. The energy consumed in this sector is divided across a large variety of processes such as assembly lines, heating or lightning. Transportation encompasses the electricity used in moving goods and people, by land, air, water or pipelines, $26 \%$ of the worldwide energy demand is used on transportation. The buildings sector encompasses the energy used in places/buildings where people live, work or obtain services, this sector is divided into two sub sectors (residential and commercial buildings), and it uses $22 \%$ of all energy produced. The residential sector is composed of the energy consumed by 
households excluding transportation, and in this sector the energy is used by appliances for heating, cooling and lighting among other commodities in the household (14 \% of total). The commercial sector combines energy used by enterprises and public administration branches in providing their services, (for example, schools, hotels or office buildings) like in the residential sector energy is consumed by heating/cooling systems, lighting and different equipment than that which exists in the commercial buildings ( $8 \%$ of total).

Since this thesis focus on the impact of the domestic energy consumption in the environment, in the next in the chapter we discuss this sector in detail.

\subsection{Domestic energy consumption}

As mentioned in the previous section, household energy consumption accounts for $14 \%$ of the total worldwide energy consumption. If we consider that humans can control up to $50 \%$ of the electricity consumption in a building (Schipper et al., 1989) the potential for saving if end-consumers reduce their electricity consumption is noticeable. These savings have an impact at the individual level (reducing the electricity bill) and on a bigger scale worldwide. Despite the efforts to develop more energy efficient appliances, households now own more appliances than they did in the past. Small appliances are responsible for over $50 \%$ of the total household consumption nowadays (Wallenborn et al., 2011). The increase in the number of small appliances used hinders an individual's control of their consumption, since the energy used is now divided among an increasing number of devices.

Furthermore, studies (Attari et al., 2010; Chetty et al., 2008) have shown that consumers are in fact concerned about the consequences of their actions on environmental sustainability. However, they are unaware of the impact of their daily activities and more importantly, are unsure as to how they can change their behaviour to reduce energy consumption. On the other side there are the developments of the electric grid into the Smart Grid which aims at introducing the electricity grid (a highly analogue system) to the digital age. This transition means that a great amount of data will start to be available, data such as city/neighbourhood/individual consumption, will be frequently updated and provided as part of the demand response or curtailment techniques. All this data will also provide opportunities for electricity feedback research and a wider dissemination of eco-feedback solutions.

These advancements motivated companies and researchers to develop technologies that present users with information about their consumption. These technologies were named eco- 
feedback technologies and it is in this concrete field of contributions of this thesis. In the next section we present the evolution current state of the art and future steps of eco-feedback technologies.

\subsection{Eco-Feedback}

Froehlich describes eco-feedback "as the technology that provides feedback on individual or group behaviours with a goal of reducing environmental impact" (Froehlich et al., 2010). This definition has been used by different researchers to include distinct work/products - for example commercial off-the-shelf equipment (Miller and Buys, 2010), low technology systems (Moere et al., 2011), abstract representations of energy use (Rodgers and Bartram, 2011) or public displays (Bird and Rogers, 2010).

Eco-feedback can be traced to as early as the 1970's starting with Kohlenberg study in which he used a light bulb that was switched on every time the household reached peak consumption in order to alert individuals to reduce their consumption. As results this early work was successful at reducing household consumption (Kohlenberg et al., 1976).

Since Kohlenberg experiment eco-feedback as a research area as grown significantly. Advances in industry meant that commercial devices were becoming easily available for studies. On the academic side studies have been conducted, and custom made software/hardware systems developed to visualize and study energy consumption (e.g. (Broms et al., 2010; Filonik et al., 2013)). In the last decade academic research in this area has conceived and proposed many agglomerated strategies and design strategies for the implementation of eco-feedback systems and studies (e.g (Froehlich et al., 2010; Pierce et al., 2008; Pierce and Paulos, 2012a). A parallel research path which has also expanded once eco-feedback disseminated is aimed at understanding how these system were adopted by individuals their families and their impact in other variables besides the energy consumption, such as routines or the household dynamics (e.g (Strengers, 2011). Currently eco-feedback research in Academia continues to evolve as the energy industry evolves, testing new concepts to control/visualize resource consumption or anticipating future trends in the industry (e.g. (Rodden et al., 2013; Simm et al., 2015).

Today eco-feedback is a mature research field with contributions from several fields of science. In the domain of Human-Computer Interaction the main international venues, like CHI, INTERACT, DIS and Ubicomp, devote specific sessions to sustainability and eco-feedback. 
In the scope of this thesis work we consider eco-feedback as a hardware or software system or any technology-aided intervention that informs and/or educates individuals about their resource consumption with the goal of reducing it. This includes not only systems that aim at reducing the energy consumption (i.e. the monthly bill), but also systems that try to move the consumption to different time periods, by educating consumers or automating activities in the house to reduce the impact on the environment (e.g. (Fischer et al., 2013)).

\subsection{The SINAIS Research Project}

Part of the work presented in this thesis document (sections 4.3.1, 4.3.2 and 4.3.3) stemmed from the FCT funded SINAIS research project ("Sustainable Interaction with social Networks, context Awareness and Innovative Services" CMU-PT/HuMach/0004/2008). The project resulted from a collaboration between the University of Madeira and Carnegie Mellon University, and which aimed at building technologies that motivate sustainable behaviours.

The SINAIS project was situated in the multi-disciplinary field of Human-Computer Interaction (HCI) and had three major focus: (i) the use of sensors and machine learning to monitor and make sense of human behavior; (ii) the application of motivational theory to intentionally influence people's behavior; and (iii) the use of a design mode of inquiry to address “wicked problems," such as sustainability.

The work described in this thesis was part of the Home consumption work package which explored the use of multi-sensor systems in conjunction with contextual inquiry HCI techniques to detect and understand significant human activities related to resource consumption in a domestic environment.

\subsection{Organization of the document}

The rest of this document is organized as follows. In the next chapter (chapter 2), we present the problem statement and research questions of this thesis, next (chapter 3) we review the literature for our work; this chapter is organized in five sub-topics: Smartgrid (section 3.1) Sensing Technologies (section 3.2), Design of eco-feedback systems (section 3.3); Ecofeedback display medium (section 3.4), Strategies to reduce consumption (section 3.5) and Effectiveness of eco-feedback over time (section 3.6); at the of this chapter we present a summary table of eco-feedback studies performed in the wild (section 3.7). The following 
chapter, Research Studies (chapter 4) is where we present the practical work conducted to inform this thesis, firstly we describe the location where the studies were performed (section 4.1) and the hardware research platform used (section 4), and two exploratory studies performed that informed the core practical work of this thesis (section 4.3). The last five subsections describe the five studies which contain the core contributions of our work to the field (sections $4.4,4.5,4.6,4.7$ and 4.8 ); each one of this studies is described starting by its motivation, implementation, evaluation and conclusions. In the subsequent chapters, (chapter 5) we present an analytic summary of all the studies performed in this thesis, comparing aspects of our system with the scale proposed by (Froehlich, 2012). The last chapter (chapter 6) presents the conclusions, future steps in our research and possible future studies extensions and implications derived from our findings. 


\section{Problem statement}

In this chapter, we present the problem statement of this thesis, detailing the main motivation for our work. The points made in the chapter can be traced to each one of the practical studies presented throughout the thesis. Firstly, we present the problem statement, then our approach to understand and address the problem and lastly we summarize the contributions.

\subsection{The problem}

The potential impact of eco-feedback technologies are starting to be questioned (Brynjarsdottir et al., 2012; Holmes, 2007; Peschiera et al., 2010). In particular, the adoption of these eco-feedback systems by individuals and their families. We define adoption of ecofeedback systems as the way these systems are used over time. Peschiera reports that after a certain period of usage of the eco-feedback devices, user consumption relapses to values in existence prior to the study (Peschiera et al., 2010). Holmes also reported a similar phenomenon in (Holmes, 2007) where users gradually returned to their previous behaviours when ecofeedback was less frequent or absent. This phenomena is called relapsing (or the relapse effect).

The relapse phenomena relates to the novelty effect found in consumer electronics. The novelty effect can be shortly summarized as consumers decreasing the interest in a product after the purchase. However, the relapse effect found in eco-feedback devices, includes also a change in behaviour of the users which compromises the purpose of the technology itself over time.

The goal of this thesis is to research the novelty effect in eco-feedback systems and, in particular, how that results in relapsing phenomena.

There is evidence of relapsing effects in other research fields related to individuals' behaviour change. For example, in personal health research, a field which looks at individuals' behaviour change related to adopting a lifestyle that would benefit their personal health (e.g. quit smoking, or exercising). Research has shown that simply keeping track of individuals' behaviour can be used to steer behaviour to the desired direction. However, the effect of these approaches tends to slowly return to pre-feedback values if the self-monitoring/tracking stops. Brynjarsdottir (Brynjarsdottir et al., 2012) states that: "self-monitoring works, (...) only as long as the individual's' self-monitors". This paradigm of self-monitoring and behaviour change 
relates to individual energy monitoring, since it also relies in a mechanism that requires that individuals track their (or their household) behaviour and taking the correct steps to change it.

This way, we hypothesize that there is a high probability that the effect of tracking individual electricity consumption is no longer effective, when users stop monitoring their consumption. In other words eco-feedback systems only work as long as they are effectively used.

However, only sparsely research studies have explored the amount of interaction/interest in the eco-feedback devices as a variable in the evaluation and discussion of such systems e.g. (Brynjarsdottir et al., 2012; Erickson et al., 2013; Gamberini et al., 2012b; Peschiera et al., 2010) Brynjarsdóttir et al (Brynjarsdottir et al., 2012) has also observed the relapse effect when reviewing work for the analysis of persuasion strategies for sustainability. The authors refer that "Little evidence for long-term behavioural change is offered in any of the papers we reviewed. The typical duration of a field study is 3-4 weeks, which is likely not long enough to go beyond novelty effects". Furthermore, the authors refer that from the reviewed work (60 articles) only two of the studies are longer than one month, one lasted for five weeks and the other for three months. Still those papers do not mention long term effects of the techniques employed in the study. This relapse effect holds great importance for the success (in terms of savings) of ecofeedback systems since the 5\% to 15\% savings reported in literature (e.g. (Fischer, 2008; Pierce et al., 2008)) can only be truly significant if these savings are continued over a long period of time.

The long term usage of eco-feedback systems is a research topic which was not completely explored in eco-feedback research, in particular in the area of human-computer interaction for which this thesis contributes. As the field that studies how people interact with the fast paced evolution of computing technologies, human-computer interaction (HCI) research tends to favour short-term studies of new and emerging technologies and less on the long-term effects of those technologies. In the following summary we sustain this claim with respect to the state of the art in the eco-feedback literature.

From approximately a hundred references reviewed in this thesis, forty-five articles clearly stated that user studies were performed. Out of those forty-five studies twelve, were actually in-the-wild studies in which participants used a system for more than one month.

In-the-wild studies are characterized for researching how technologies are adopted/integrated by people in their everyday lives. A important concern when performing in- 
the-wild studies is to take into account user requirement, either for technologies that will introduce new practices or complement pre-existent practices. These studies move away from lab studies, which are usually utilized to produce design implication or system guidelines (Chamberlain et al., 2012).

Considering the twelve studies that were actually performed in the wild and lasted longer than one month, seven did not considered the amount of interactions with the system as a variable present during the evaluation. Finally, we isolate the five longer-term studies, which mentioned logging the interactions with the users during the evaluation. Brooms et al referred that the interaction had two phases: one more active, where households explored the consumption of their appliances with the help of the electricity meter; a second phase when the consumption settled the system was used in a lesser extent (Broms et al., 2010). Erickson et al. (Erickson et al., 2013) evaluated a website deployed in a city-wide experiment. Regarding the interaction with the system the authors referred that $35 \%$ of the residents logged into the system at least once, and that's the only time the long term interaction is mentioned in the article. Usage of the system data was also collected in the Dashboard display developed by Filonik et al. (Filonik et al., 2013). However, the authors did not take into account those variables, arguing that they were not relevant for the type of the evaluation present in the article. Gamberini reported (Gamberini et al., 2012a) the relapse effect, where interactions with the system decreased from 20 interactions per day in the first day to eight in the last one (however the author do not report how the interaction actually evolved during the study). At last, Kjeldskov (Kjeldskov et al., 2012a) also logged users' interaction in their study, however the authors only refer that the interactions dropped and do not mention how this dropped happened. Figure 1 summarizes the abovementioned studies subsets.

The body of work presented above demonstrated that, although several authors have considered the interactions with the system as a variable to collect during the evaluations, the way the interaction evolved over time has not been a major concern in eco-feedback research. That aspect of the studies is generally a footnote in the discussion or results section of the abovementioned articles.

We argue that there are few studies that evaluate feedback systems "in the wild" within real homes with families, this necessity has been identified HCI researchers, not necessarily working on eco-feedback e.g. the research into the Wild workshop as part of ACM's Designing Interactive Systems conference (Chamberlain et al., 2012). 
The hypothesis of this thesis is that the usage of eco-feedback devices relapses after a period of time. This claim is tested through five long-term studies in-the-wild where families were able to use eco-feedback systems in their daily lives.

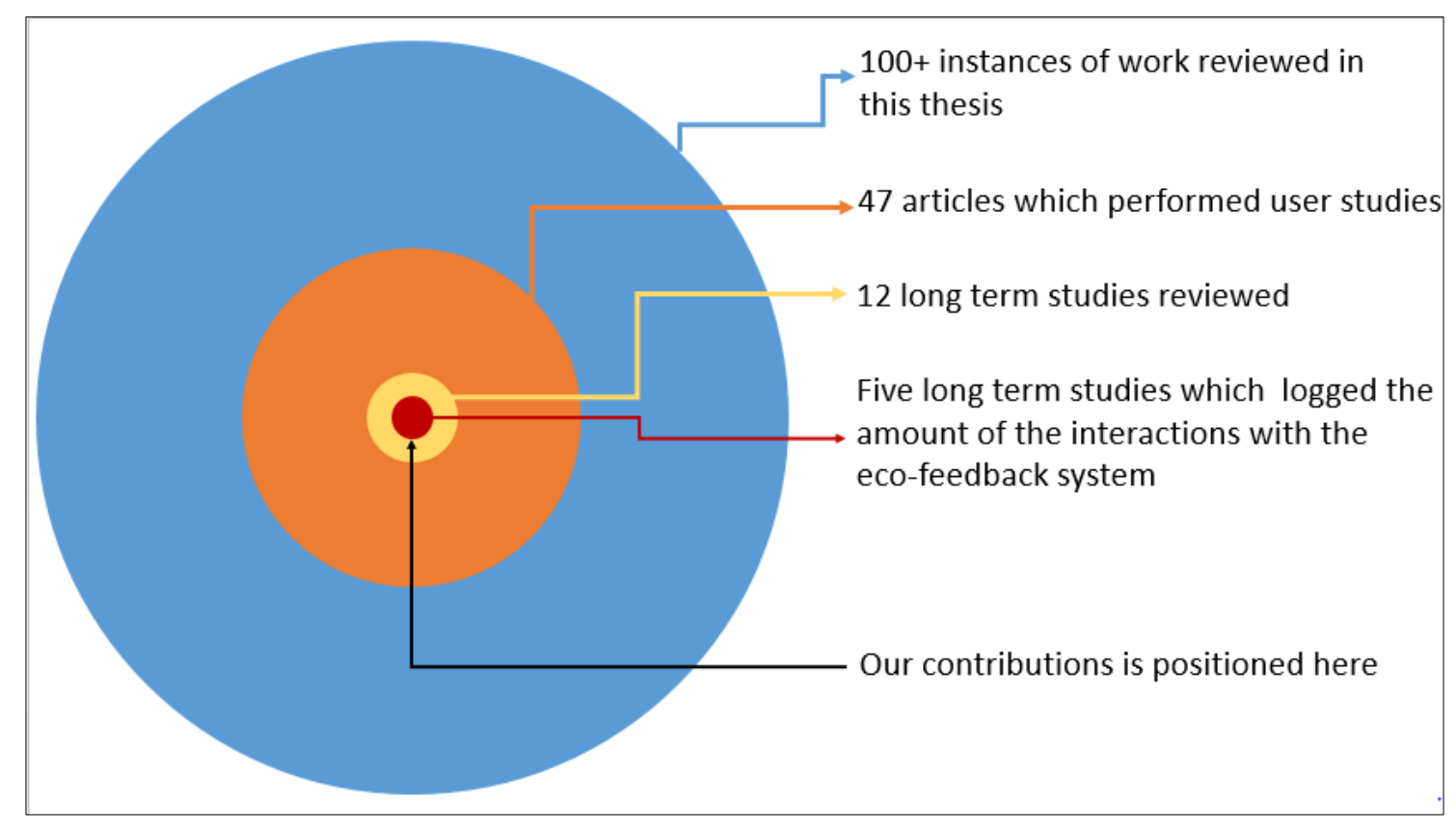

Figure 1:Graphical representation for the different subsets of studies discussed in the problem statement.

\subsection{Proposed solution}

This research aims at understanding the relapse behaviour in domestic eco-feedback systems through long-term deployments that explore different factors that might influence household energy consumption. Building on current literature and industry efforts, we implemented and tested several eco-feedback systems.

Our prototypes explored different dimensions and variations of eco-feedback, including location (fixed or mobile), design (bar charts or abstract visualizations) and complementary information (mapping consumption and production). Through this research we seek to create eco-feedback systems that are well-understood by families and to understand the effect of the abovementioned dimensions in the long term. All studies presented in this document were performed in-the-wild, and the evolution of the interaction with the systems will considered in all of our studies, however this evaluation also took into account other metrics pertinent to energy consumption, like change in the consumption behaviours. 
As previously mentioned the goal of this thesis is to understand the long-term adoption of eco-feedback devices. This assessment will firstly observe for the novelty effect on ecofeedback systems, and in a second stage verify if those effect result in a relapse of the electricity consumption.

However, that is a very wide goal, and we argue that it would be very hard to perform that assessment without actually deploying and testing eco-feedback devices. This way, this thesis also contributes in other specific strategies tested in concrete studies.

The two first studies with the SINAIS Power Meter were performed to objectively evaluate the long-term usage of eco-feedback devices (sections 4.3.2 and 4.3.3). After analysing those studies results, we investigated other feedback strategies and implemented a paradigm shift in our system, focusing in displaying individual's' consumption as the impact in the environment (sections 4.4,4.5, 4.6, 4.7 and 4.8).

The actual motivation and decisions specific for the abovementioned studies will be presented throughout this document. Nonetheless, we can identify the goals of this thesis as:

1. Assess the usage of eco-feedback system in the long term.

2. Test displaying the individual's' impact in the environment as means of feedback.

a. Abstract representation of individuals' impact in the consumption.

b. Accurate representation for individuals' impact on the environment.

More concretely our work aims at answering the following research questions:

RQ1: Can we detect the novelty effect in eco-feedback systems deployed in the wild?

RQ2: Is the novelty effect related to variables such as location, representation and information?

RQ3: Can the novelty effect be related to relapsing behaviour prior to the exposure of the ecofeedback?

\subsection{Practical work}

In this section we present (see Table 1) a picture/screenshot of each system developed and tested as part of this research thesis. In the next section we will briefly describe each one of these studies (section 2.4). However, each system and its evaluation will be presented in detail in chapter 5 this document. 
Table 1: Systems developed in the period of this thesis.

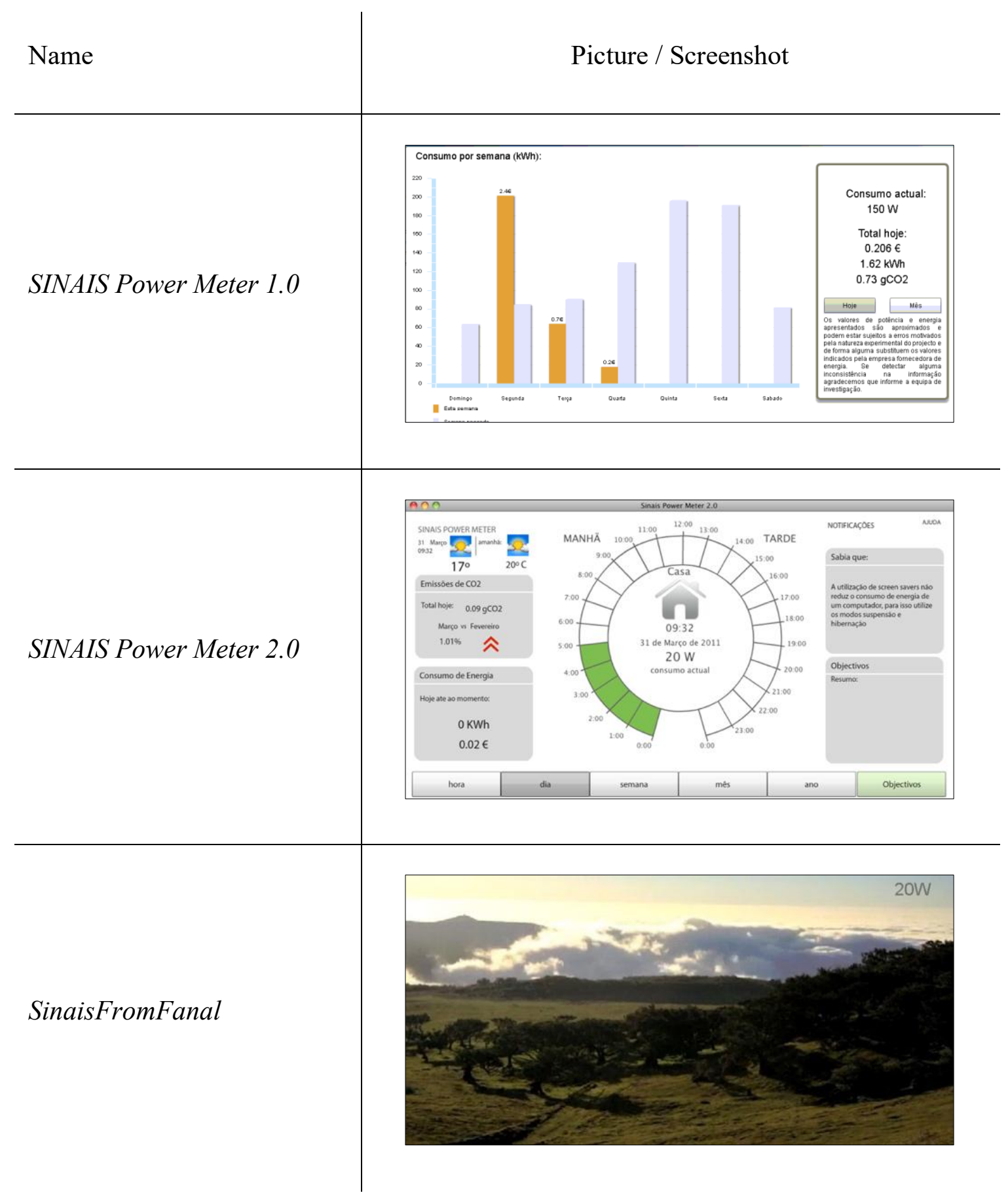




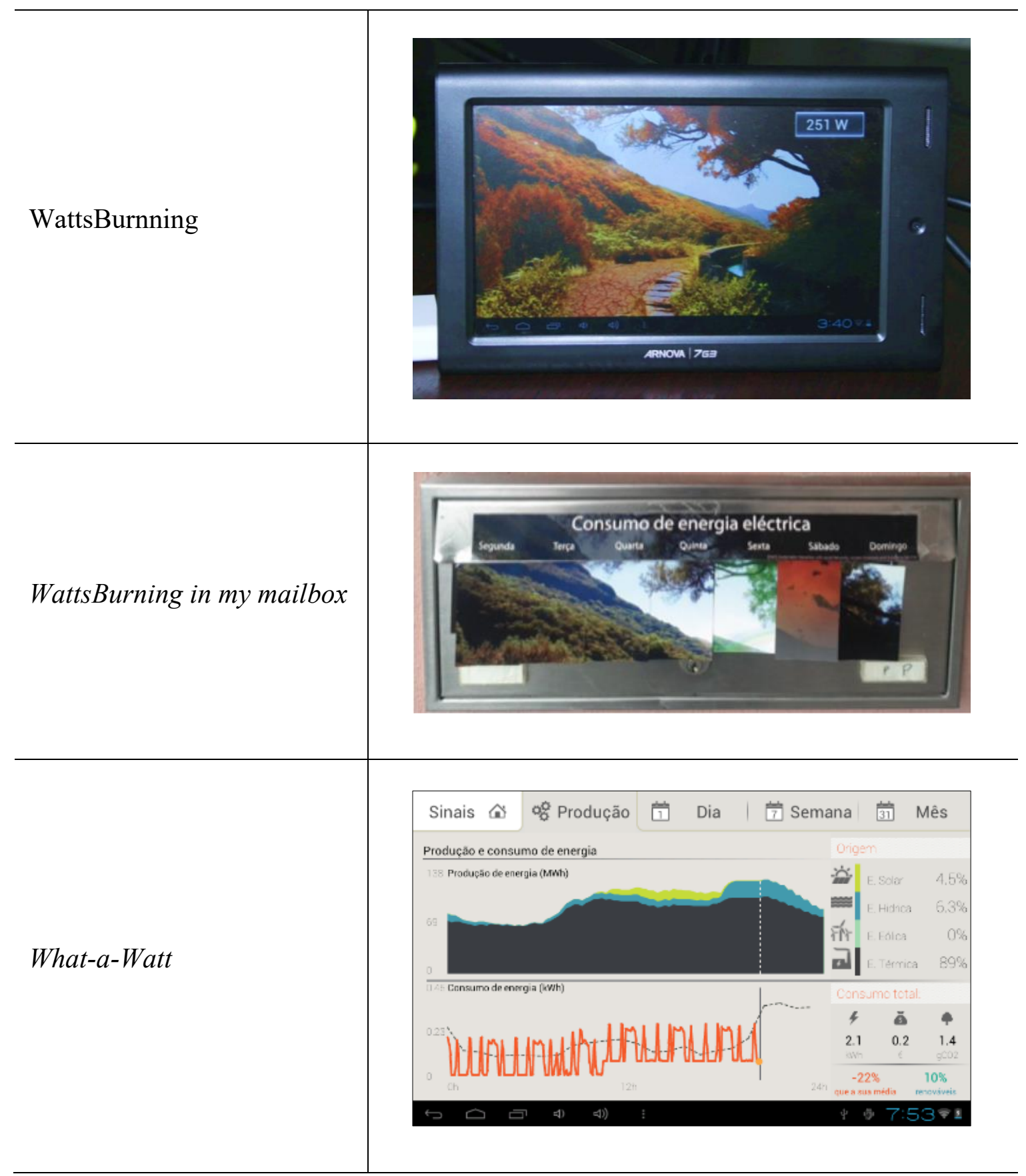


What-I-See

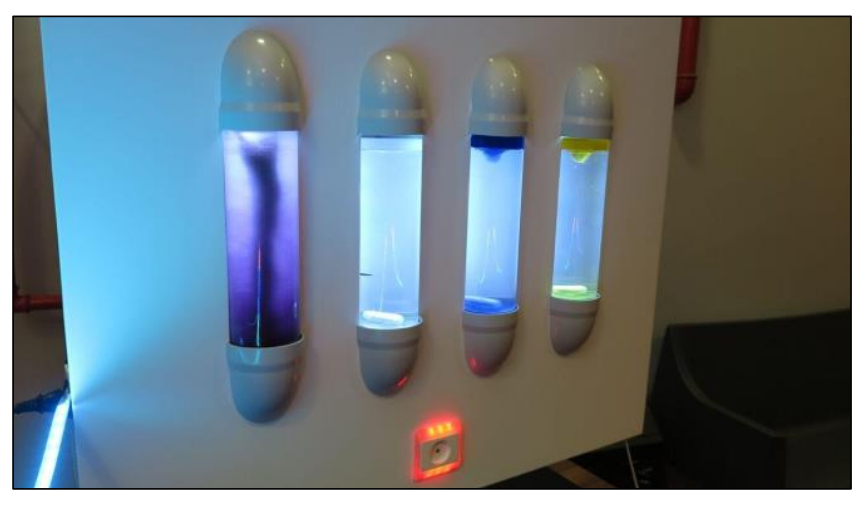

\subsection{Studies timeline}

Figure 2 presents an overview of how the different studies described in this thesis took place over the period of four years.

Our practical work started in July 2010 with the first deployment of the SINAIS Power Meter. This system used a simple graphical representation of consumption and was implemented in Adobe AIR running on a netBook that was also collecting user interactions (through the webcam) and acquiring the electrical signals through the soundcard of the netBook. The SINAIS Power Meter 2.0 was an update to the first system, in which the front end of the system was completely redesign based on a set literature good-practices. Our third eco-feedback study used the SinaisFromFanal system which was still running in the netBook, however in this study we deployed a completely different visualization based on the local landscape. The design rationale was kept in the WattsBurning study, which ran on a 7"' tablet. The WattsBurning in my mailbox study was completely different from our work up to that point, it lasted for one week, and in it users shared their household electricity consumption using a set of magnet placed in their mailbox. Our research path proceeded with the What-a-Watt study with exposed consumers to the source of their electricity, this study used a tablet application with a custom made application. Finally, our last practical study was the evaluation of the Watt-I-See an installation which presented with individuals with source of their electricity in real-time.

The motivation, implementation and results of the abovementioned studies will be described throughout this thesis in the sub-chapters 5.3.2, 5.3.3, 5.4.1, 5.5.1, 5.6.1, 5.7.1 and 5.8.1. 


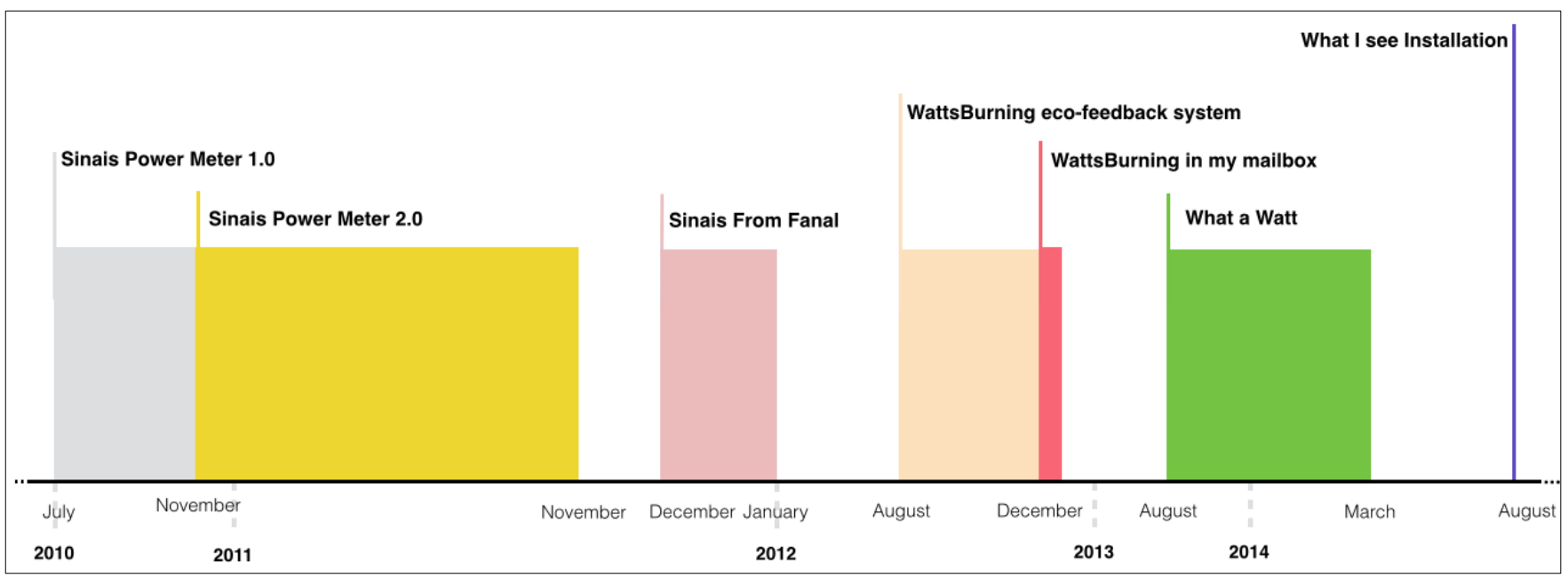

Figure 2: Timeline which places our practical studies along the time period of this dissertation. 


\section{Background: A Survey of Energy Monitoring and Eco-Feedback Technologies}

This chapter describes the literature related to eco-feedback systems Section 3.1 starts with defining more general concepts in which eco-feedback systems are usually considered, like the Smartgrid and Smart cities, which are at the basis of much of the reviewed work. Next, in section 3.2 several approaches for measuring resource consumption are presented. In section 3.3, we describe the results and design implications of a set of studies with eco-feedback devices. Our review continues by investigating the physical aspects of eco-feedback devices in section 3.4. Next in Section 3.5 we present techniques that have been used to motivate consumers to reduce their environmental impact, such as Motivation Theories, Public Displays and Artistic/Abstract eco-feedback. The Energy Production section describes studies that have used the origin of the energy in the feedback system. The following section 3.6 aggregates and describes the hardware that was used in all the reviewed work. The final section (section 3.7) of this chapter aggregates several observations of the effectiveness of eco-feedback systems over time.

The topics referenced through the analysis of the related work influenced our work in various ways, they added to our provided motivation, inspired solutions and allowed us to compare our results with the state of the art. For each one of the next sections, we state how each topic affected our work.

\subsection{Smartgrid}

The commonly called Smartgrid is of the most visible part of the constitutional and governmental side of the sustainability effort. Eco-feedback devices are the last component between utilities and consumers in the effort for making the electric grid smarter. In this section we present studies that took advantage of the already deployed Smartgrid systems, or studies that tested concepts to be implemented in the envisioned Smartgrid.

The Smartgrid consists of the employment of a series of technologies such as high frequency sensing and two-way communication between consumers and providers with the goal 
of making the production, distribution and consumption of energy more effective. Predictions calculate that savings in $\mathrm{CO}_{2}$ emissions of between $5 \%$ and $16 \%$ can be achieved with techniques and technologies such as advanced metering, dynamic pricing or automating technologies (Hledik, 2009) . Even though the Smartgird development is at an early stage which just a small number of locations/providers e.g. Hydro One ("Demanding standards," 2007), E-Energy ("EEnergy," 2007), researchers have already started to study how consumers might react to the different technologies employed.

Fischer et al. (Fischer et al., 2013) built a system for personalized energy usage recommendation, more specifically personalized recommendations about energy tariffs. For this, the system uses a series of algorithms for load detection and usage prediction. As a particular example, the system detects deferrable loads (which are non-overlapping uses of the washing machine, clothes dryer or dishwasher). The consumption profiles are then used on a website to do a series of comparisons/estimations of the energy cost depending on the provider and time of day (the system could estimate the cost of changing a deferrable load to a period where the energy cost was smaller). The system was evaluated using ten participants, all of whom reported that they would use the system again. For nine of the participants, the agent switch found cheaper tariffs with annual savings of between $£ 35$ and $£ 391$. However the research team found some barriers to the adoption of the system. For example, the need for more convenient switching between tariffs, and clearer contracts with the provider.

Rodden et al (Fischer et al., 2013) explores how consumers might react to the current developments in the smartrid, and mandatory smart meters in every household. The team organized a focus group with 17 participants, who were shown a series of video sketch animations that presented several aspects of the electricity production, distribution and consumption, divided into two timeframes the near-future and the future. The authors note that given the number of entities involved in the grid, a holistic presentation was needed. Additionally, any smart meter or agent installed in a household will have to address issues such as making it explicit as to who owns and operates it. Regarding automated agents (that for example can turn off a device when the demand is very high) the article argues that such systems should make their action understandable and accountable by clarifying what triggered the automated action. Figure 3 presents an example of a smartmeter already deployed is several households in Europe.

The last studies of our practical work (section 4.7 and 4.8), like the work presented above were partially motivated by the possibilities of the proposed new smartgrid. 


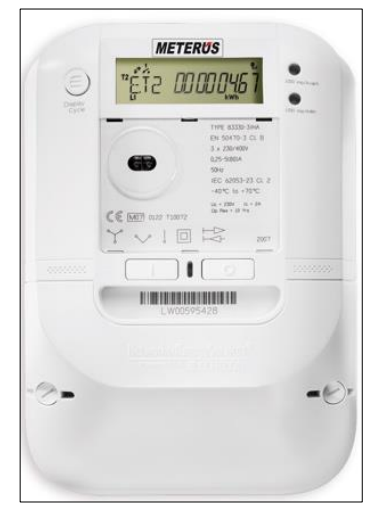

Figure 3: Example of a smart meter based on Open smart grid protocol (OSGP) in use in Europe that has the ability to reduce load, disconnect-reconnect remotely, and interface to gas and water meters (EVB Energy Ltd, 2008).

\subsection{Sensing Technologies}

Measuring resource consumption is a key element of eco-feedback systems. There are several research and commercial systems for measuring resource consumption, and in particular electricity consumption which is the main focus of this thesis. The most common systems are energy meters which utility companies use to bill consumers. However, despite the new smart meters these sensing technologies are based on regulatory billing guidelines and therefore cannot provide detailed or disaggregated consumption information. This means that researchers often have to develop custom-made sensing solutions in order run eco-feedback studies. Monitoring solutions range from simple approaches likes querying the user for input to complex systems that use signal processing and machine-learning algorithms to infer individual appliance consumption. Next in this chapter we present a series of approaches for measuring energy consumption. Although our work does not make contributions in this field, we did review research and commercial approaches suitable for our eco-feedback device, this way the work presented below allowed us to extract features/solutions from state of the art eco-feedback devices which were then used or adapted to our systems.

A simple technique that does not require a complex infrastructure is for consumers to self-report their energy usage or actions during their everyday routines. This technique is used in the Stepgreen social network (Mankoff et al., 2010), in which users report and discuss actions regarding sustainability. Today, however, researchers have developed sensors and frameworks for energy consumption monitoring that can provide accurate data that can then be used in deploying eco-feedback systems. Regarding electricity consumption, one of the most used and 
cost-effective approaches is the Non-Intrusive Load Monitoring (NILM) (Hart, 1992), which is a system that reads data from one point (normally in the main power feed of a building) and monitors the consumption of a household or a set of appliances. This was the approach used in the sensing infrastructure supporting our research.

NILM is built on the assumption that every change in total consumption in a building happens as a consequence of an appliance changing its state (for example a light bulb is turned on, or the fridge motor starts working). These changes in states are commonly called power events. The total consumption is analysed using sophisticated signal processing and machinelearning algorithms, with the goal of inferring the disaggregated per appliance consumption. NILM is a low cost alternative to intrusive technology, and its potential is growing to other domains. For instance, in (Cohn et al., 2010) Cohn et al. developed Gas Sense, a single-point approach to measuring gas flow with an acoustic sensor.

A similar solution was implemented to measure water consumption, Hydrosense (J. E. Froehlich et al., 2009) uses one pressure sensor and it allows the system to identify which water faucet is being used and to estimate how much water is consumed (Figure 4 left). The sensor is connected to a faucet in the plumbing system of the house and when a valve switches (for instance a bathroom faucet or a mechanical valve in a dishwasher), a pressure change will occur and a pressure wave is generated in the plumbing system that can be sensed anywhere in the house. The characteristics of this waveform will be different for every existing valve, which makes it possible to once again apply NILM techniques to classify events. The work of Zeifman and Roth (Zeifman and Roth, 2011) presents a good summary of the research done in this field and points out opportunities for future research. Other authors have achieved similar results with different approaches. Patel et al (Patel et al., 2007) implemented a system that measures the electrical noise of each appliance from the voltage measurements in outlets so that it could detect and classify the behaviour of most appliances. Later the same author criticized the previous work mentioning problems such as the computational expense of analysing the noise, and presented ElectriSense (Gupta et al., 2010) which focused on sensing very high frequency electromagnetic interference which is present in most modern consumer electronics (Figure 4 right).

Kim et al (Kim et al., 2009) developed the ViridiScope, an energy-monitoring tool that combines different types of indirect sensors (light, acoustic and electromagnetic) with the total consumption load of the household with the goal of monitoring the consumption of all the appliances. The proposed system does not require calibration after the installation. The authors argue that the inputs from the abovementioned sensors are capable of detecting the different 
states of all appliances (for example to detect if a light bulb is ON the light sensor is enough, however to detect the different states of a washing machine all 3 sensors are needed). The authors conclude with a series of evaluation sessions in which the average consumption error is less than $10 \%$.

In (Weiss et al., 2009), Weiss describes his architecture for measuring energy consumption, which is composed of an off-the-shelf meter, a gateway with an Application Program Interface (API) that provides access to the meter data, and an application on a mobile device that provides real time feedback on consumption. The mobile application displays data such as total load compared with the household baseline and historical data presented in charts. Additionally, the application also allows users to measure the consumption of a particular device by calculating the difference in the load (however there are some devices that consume a significant part of the total but can't be measured in this way).

Hay et al proposes the global personal meter that records and distributes personal consumption between individuals (Hay and Rice, 2009) the authors present how such system could be developed, as well as the challenges and opportunities of such systems. The same authors study the concept further, by exploring how sensors installed in a building can help to distribute the consumption between occupants. The paper proposes a set of techniques to correctly measure the consumption per occupant and the authors explore how common spaces or individual appliance consumption can be divided by distributing sensors widely throughout the building infrastructure.

Additionally, there are an increasing number of commercial applications that provide real time energy monitoring. These solutions range from low-cost single-outlet (Kill-a-Watt and Watts Up), to medium cost whole-house power consumption (CurrentCost3, TED4, Efergy5, Owl6, etc.) and to higher cost ambient feedback solutions (Wattson7, Energy Orb8). Through these solutions, the feedback is often presented as raw energy use (e.g., Watts), personal cost (e.g., money), or environmental impact (e.g., $\mathrm{CO}_{2}$ emissions). Besides the commercial aspect of such systems, these platforms have also allowed researchers to shorten the development time needed for eco-feedback studies, by, for example, building hybrid systems that combine off-theshelf hardware with custom made hardware like in (Foster et al., 2010). 

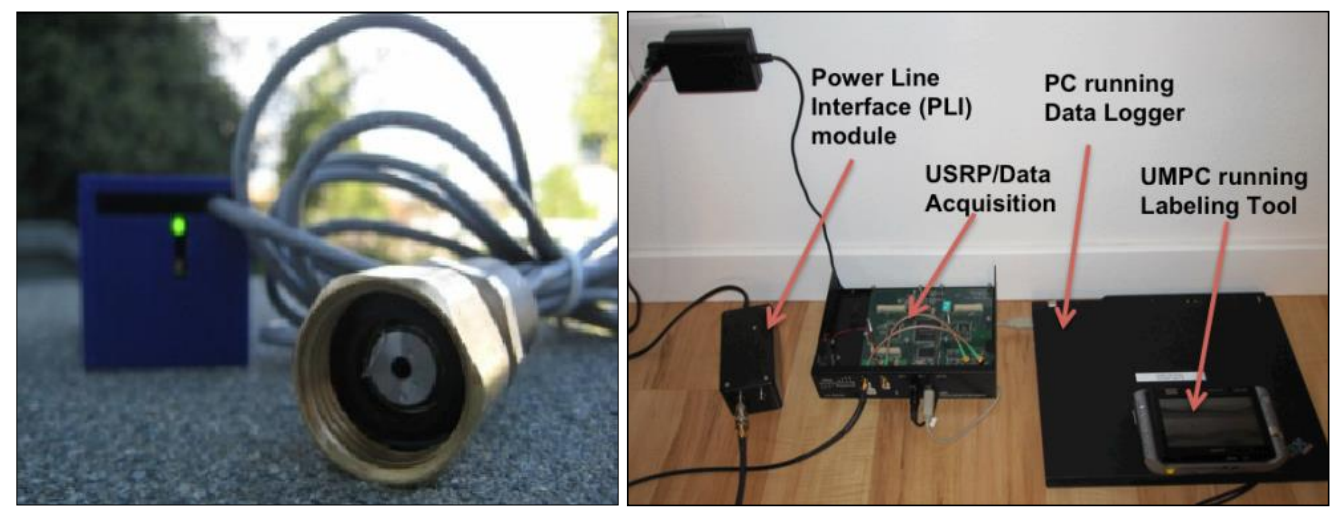

Figure 4: Left: HydroSense sensor system (J. E. Froehlich et al., 2009) Right: Electrisense system

(Gupta et al., 2010).

\subsection{Design of eco-feedback systems}

The research literature is abundant in design techniques and guidelines to implement ecofeedback systems (Froehlich et al., 2010; Mills, 2002; Motegi et al., 2003; Strengers, 2011) and in theoretical models that can be used to motivate behavioural change (Abrahamse et al., 2007; Barreto et al., 2011; Consolvo et al., 2006; Scott et al., 2011). Techniques such as displaying real-time, comparison, historical, and carbon emission information are widely described as the type of information users like most. For example, comparing individual consumption with others might also help consumers to benchmark their resource use (Chetty et al., 2008; Petkov et al., 2011). Two major aspects have been identified as crucial in the design of eco-feedback systems (Pierce et al., 2008), the type of data presented (e.g. electricity consumption, water consumption), and the visualization techniques used to present this information (e.g. charts or numbers).

The actual design process of eco-feedback systems follows various methods that common in Human-Computer-Interaction research. Prototyping and iterative methods that utilize user input are widely present in the reviewed work, the different prototypes are usually refined with small pilot studies, semi-structured or contextual interviews (e.g. (Fischer et al., 2013; Froehlich et al., 2012; Mankoff et al., 2010)). However, the novel nature of these systems has also benefited from design methods used to uncover new ideas, such as for example focus groups, workshops, or input from experts in the area (e.g. (Broms et al., 2010; Fischer et al., 2013; Foster et al., 2010) ). 
Here we present different studies that recommend particular design strategies. The following studies disclose concrete design implementation as well as qualitative and quantitative data of consumer usage of eco-feedback systems. The results and recommendation of the studies presented in this chapter were used as inputs in the design process of our own eco-feedback devices presented later in this document. Furthermore, this is a sub-field which we aim at contributing with the results of the custom eco-feedback devices developed in this thesis.

Jakobi and Schwartz (Jakobi and Schwartz, 2012) developed a Home Energy Monitoring System that was installed in nine households. The system had a tagging mechanism, which allowed users to assign the consumption to four categories: activities, rooms, appliances and individuals. The results showed that participants often tried to justify their consumption by referring to the context in which it took place. The deployed prototype could only measure nine appliances at a time. Participants often changed the configuration and the places where the smart plugs were first installed. During the tagging process participants often wanted to reconstruct usage scenarios, they remembered specific usage situations with unknown consumption, or triggered by the feedback mechanism, where the amount of consumption raised curiosity concerning the root of the consumption. The authors argue that it is important to let users build their own understanding and language of how to organize tagging.

Gamberini et al.(Gamberini et al., 2012a) developed a game that promoted electricity conservation. This game provided appliance-level consumption, which allowed the system to provide "smart advice" regarding the usage of any particular appliance. The authors observed a high level of engagement with the system. Participants continued to use the game throughout the whole study and the consumption of specific appliances dropped $38 \%$ on the day after the advice was triggered (Figure 5 right).

Ueno et al (Ueno et al., 2005)implemented an electricity monitoring system that was able to monitor whole-house as well as up to 18 individual appliances. They deployed the system in ten households, in total there was a reduction of $9 \%$ in consumption. However the authors found bigger savings were made on particular appliances, for example space heating, where savings ranged from 20\% - 45\%. Tae-Jung Yun implemented and tested an Energy Consumption display (Figure 3 middle) in 8 households over a period of 3 weeks (Yun, 2009). The study had two conditions: half of the sample used stationary feedback displays and the other half used portable ones. The author found that after a period of exploring and experimentation portable devices were eventually used as stationary. On average, participants reduced their consumption by $11 \%$ and the impact was bigger on participants who reported a lower awareness of energy use. High awareness users "asked" for more information such as room/appliance level 
information, or comparison with different households. The author also found that the system supported goal-setting within the participant's consumption even though there was not any feature on the application intended to function that way.

The Dubuque electricity portal (Erickson et al., 2013) was a website that provided inhabitants of Dubuque city in the United States with feedback on their electricity consumption. The portal deployed several techniques to encourage savings such as comparisons, social incentive and goal setting. In total the study, which lasted for 20 weeks, managed to reduce participant consumption by $3.7 \%$. Of the 765 houses in the deployment, $35 \%$ used the system at least once. The usage data of the portal disclosed that the consumption timeline (chart with consumption from the beginning of the year) was the most used interface feature followed by the consumption by hour and comparisons with the previous year. These were also the features that users felt were more helpful while trying to understand their electricity usage. Sixty one percent of the participants who used the system admitted to making at least one change in the way they used electricity. Finally most of the reported results build on previous results found in literature, and the authors argue that the most likely promoter for radical changes in consumption will be the shortage of resources or the price, and then eco-feedback devices will have to be part of the solution.

Froehlich et al (Froehlich et al., 2012) evaluated a set of eco-feedback design techniques for a water consumption display. The authors gathered data from an online survey with 712 answers as well as a more detailed semi-structured interview in ten households. The research team tested several design probes, with isolated aspects such as data granularity/time or measurement unit. The authors concluded that users prefer the traditional representation of information (bar charts for example) compared to less data-centric representation (for example an animation of a fish tank that would change state based on the water savings, Figure 5 Left). The research also disclosed that users valued disaggregated consumption information (by household occupant for example) over whole-house aggregated information.

The observation that consumers value the breakdown of the total consumption into individual appliances has been consistently reported in the literature. For example, Hargreaves et al. conducted a study (Hargreaves et al., 2010) in which participants used appliance-specific monitoring devices to understand how "greedy" particular appliances were. Additionally, participants without appliance-specific monitoring devices were able to estimate approximate consumption of particular devices by the difference in the total consumption load of the house. The same remark was made in (Broms et al., 2010) where consumers managed to infer particular appliances by the shape they made on the consumption chart. 
As eco-feedback technology evolves, different eco-feedback techniques emerge. For instance Strengers (Strengers, 2011) reports on the findings of an energy and water eco-feedback program with in-home displays in 26 households in Australia. After an in-depth qualitative analysis the authors proposed an alternative paradigm relying on the everyday needs of consumers, as opposed to adding another appliance to use during their everyday routines. The author exemplifies this shift in paradigm with examples such as programming sustainable action into appliances, like setting the default water temperature on the washing machine to be cold.
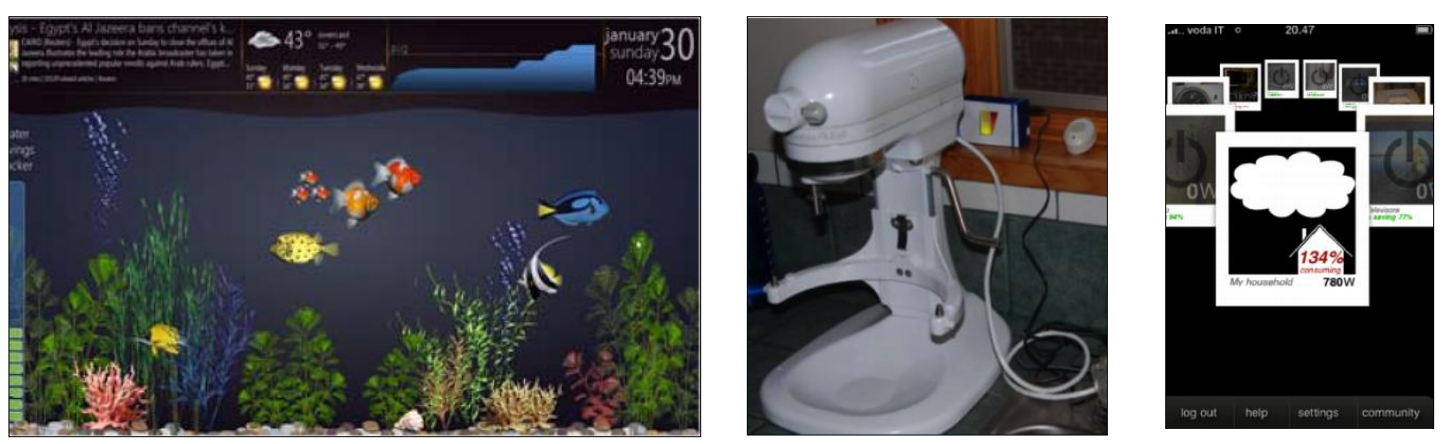

Figure 5: Left: Abstract representation for water savings from (Froehlich et al., 2012); Middle: Meter used in (Yun, 2009);Right: Screenshot of the mobile application used in (Gamberini et al., 2012a).

\subsection{Eco-feedback display medium}

By definition eco-feedback studies rely on some type of artefact that will be used to inform individuals about the impact of their behaviour. These artefacts can be as simple as a letter once a month or another type of low-fidelity feedback technique e.g. (Bird and Rogers, 2010; Moere et al., 2011), as complex as custom-built hardware/software platforms (Abrahamse et al., 2007; Weiss et al., 2009) or simply off-the-shelf devices (Figure 6). From the literature review presented here three studies used some form of low-fidelity feedback, for example a chalkboard as a public display in (Moere et al., 2011). Eleven of the reviewed studies used a custom-built framework or hardware - for example the custom feedback device used in (Broms et al., 2010). In fifteen studies the authors used custom software, for example the custom social network developed by Mankoff et al. (Mankoff et al., 2010). The increase in availability of commercial devices to monitor energy consumption also made them a reasonable choice for studies aimed at understanding individual behaviour, for example Strengers (Strengers, 2011) evaluates how a commercial meter is accepted in the household. Moreover, the increase in 
availability and capability of smartphones and their presence in everyday routines have made them suitable for feedback studies, and we verified that they were used in six of the reviewed studies - for example Froehlich et al. (J. Froehlich et al., 2009a) used a mobile phone application to study user-mobility and transportation habits. Finally there were also studies that used probes to represent feedback devices that are not currently available or too expensive to implement, for example Yun (Yun, 2009) investigates how a minimalist display could be built and how it would impact consumption at home using a probe of the envisioned hardware.

The increasing availability and significance of commercial hardware can be demonstrated by the relevance of studies which have targeted the widespread accessibility of such systems. Miller and Buys (Miller and Buys, 2010) conducted a study with seven households that were using a commercial feedback device that monitored electricity and water consumption and the study generated a set of guidelines for how such devices should be built and marketed as an appealing product for the household. The price of these devices was a concern for some residents and another concern was the lack of customer support for the used water-andelectricity meter. Additionally, the meters themselves were not very reliable, producing incorrect results on several occasions and the authors argue that the whole product supply chain should be robust and that the failure of any of its parts would be seen by consumers as a failure of the entire product. Also Pierce et al (James Pierce, 2010) reflects on the effectiveness of commercial eco-feedback devices. The authors present qualitative findings from two studies, the research disclosed that receiving the feedback increased consumer awareness of electricity usage. Furthermore, consumers adopted the notion of baseline consumption as "normal" and the authors also identified a gap between household anticipated behaviour and actual behaviour (for example some participants did not change certain routines even after learning that they were inefficient). The author concludes by outlining a set of research opportunities for residential feedback devices - for example designing systems to reduce baseline consumption instead of only being alerted when consumption is over the baseline. 

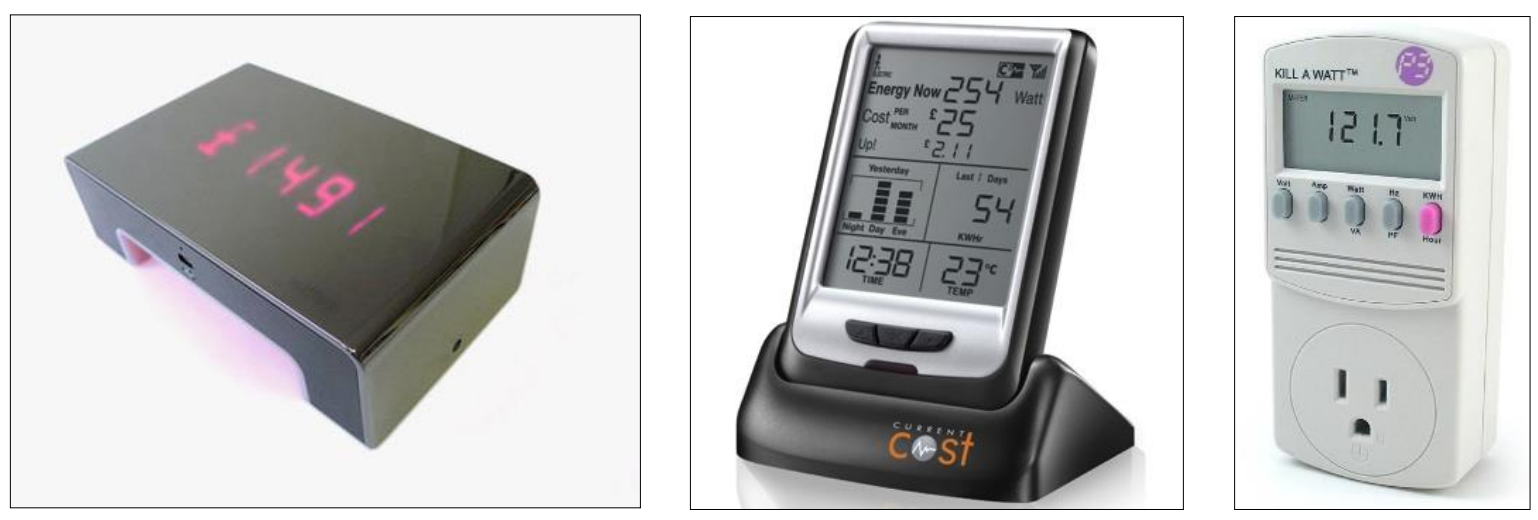

Figure 6:: Different models of commercial energy monitors, Wattson, Current Cost and Kill a Watt.

\subsubsection{An eco-feedback design space}

Recently Froehlich developed a scale that represents the design-space for eco-feedback systems (Froehlich, 2012). The scale depicts the different dimensions of eco-feedback systems as follows: Information Access; Interactivity; Display Medium; Actionability/Utility; Data Representation; Motivation/Persuasive Strategies; Comparison and social Aspects.

During our work we explored different dimensions of eco-feedback, Froehlich's framework allowed us to have a bird's eye view of our contributions. By classifying our work in Froehlich's design space we can easily identify the difference between our systems, and place our work among the body of research in eco-feedback.

Next in this section we explain in detail each aspect of the abovementioned framework. At the end of this document (chapter 6) we use Froehlich scale to summarize our contributions in terms of the design strategies used in each eco-feedback system.

The Information Access dimension is related to how the information is accessed, updated and how attention-demanding it is. This dimension contains four sub-dimensions: The update frequency is the frequency with which the feedback is updated. Spatial proximity to behaviour relates to the positioning of the feedback in relation to the place where the wanted behaviour is going to happen. Attention demand classifies how "glanceable" the feedback is, i.e. how much attention the user has to devote to it in order to obtain eco-feedback information. Effort to Access relates to how much effort the user has to make in order to obtain consumption information from the eco-feedback system. 
The Data Representation dimension, relates to how the consumption information is presented to the user - i.e. how the information is grouped, the units that are displayed and which techniques (e.g. charts, textual information) are used. This dimension contains nine subdimensions: Aesthetic describes the general appearance of the feedback, for example the tradeoffs between utility and beauty. Time Window relates to how historical consumption information is available to users, and how far the system allows consumers to consult their energy-use information. Temporal Grouping relates to how the consumption data is grouped in the time dimension, for example real-time, by hour, week or month. Data granularity refers to the resolution of the consumption data presented in the feedback (e.g. consumption data disaggregated by appliance or consumption grouped by city). Visual Complexity describes how busy/cluttered the feedback feels to the user. Primary Visual Encoding relates to how the elements in the interface are used to convey the feedback message. This metric ranges from completely textual to all graphical. Measurement Unit is the unit used to present the consumption information to the user (for example Cost, $\mathrm{kWh}$ or $\mathrm{g}$ of $\mathrm{CO}_{2}$ ). Primary View refers to the feedback modality that is used in the primary view of the system, for example temporal representation using a time series. Data Grouping relates to how the consumption data is grouped in the feedback. For example, the consumption can be grouped by appliance, activity or place.

The Interactivity dimension relates to how much the user can interact with or customize the feedback interface. There are three sub-dimensions to the interactivity dimension: Degree of Interactivity refers to the level of interaction that the feedback visualization allows. Interface Customizability describes whether the interface can be customized or not. User Additions describes whether the interface supports user additions such as annotations or corrections of erroneous sensing data.

The Social Aspect dimension encompasses strategies used to incorporate social influence in the changes of consumption behaviour promoted by the eco-feedback system. This dimension contains four sub-dimensions. The Target describes the target of the eco-feedback system. This could be individuals (for household consumption monitors) or groups of people (for example a city-consumption public display). The Private/Public variables define if the feedback was designed for public or private use. Data Sharing refers to what information from the feedback system is shared with others. Social-Comparison defines the groups of people that the consumption data is shared with.

The Display Medium dimension refers to the physical aspects of the eco-feedback system. For example, screen size and form. There are three sub-dimensions to the Display Medium: The 
Manifestation variable refers to the physical instance of the feedback - for example a phone, tablet or website. The Ambience variable describes whether the feedback system contains aspects associated with ambient displays. The Size relates to the size of the medium used to display the consumption feedback.

The Actionability/Utility dimension of a feedback system refers to how easy it is for a user to start with information presented by the system and change their behaviour accordingly so that it that impacts the environment. This dimension encompasses six sub-dimensions: Degree of Actionability, is the degree of how immediate it is for a user to know what proenvironmental action to make based on information presented by the system. Decision Support relates to whether the system supports the consumer with particular actions that will lead to a smaller environmental impact. The Personalization this variable defines to which degree the display/feedback can be personalized to address single individuals or households needs. Information Intent defines the number of actions about which the eco-feedback system can inform consumers. For example, a household electricity monitor informs the user about several different individual behaviours such as, for example, air conditioning usage. The Automation/Control element defines whether the feedback system can control other household items (e.g. lighting, doors, windows, etc.) to improve the energy efficiency.

The Comparison dimension relates to how the feedback system uses comparisons to motivate consumers to adopt more pro-environmental behaviours. This Comparison dimension contains five sub-dimensions: The Comparison-Target relates to how the comparison is made. The targets can be the same (individual self-comparison), other people, or goals/milestones. The Comparison by Time refers to which time window is used in the comparison, for example comparisons with yesterday, the past week, month or year. The Social-Comparison Target refers to which social groups are used in the comparison, Froehlich states that most of the comparisons fall into the geographically proximal, demographically similar, or users of the same social network groups. Goal-Setting Strategy describes which strategy is used to set and track the goals in the system. Difficulty to Reach Comparison Target is associated with how hard (or how perceivably hard) it is to reach the comparison target.

The Motivational/Persuasive Strategies dimension includes aspects that are also part of other dimensions such as comparison, social aspects and actionability. However, Froehlich notes that this dimension is much wider and includes techniques from persuasive technology, behavioural science, environmental psychology, game design, social marketing and health behaviour change. 


\subsection{Strategies to reduce consumption}

Understanding why consumers change their habits is a complex research topic with contributions from as diverse fields of science as psychology or economics. Additionally, motivating consumers to change their energy consumption habits is a similarly complex problem. Riche et al (Riche et al., 2010) describes a three-stage approach for a change in energy conservation behaviour: raise awareness, inform of complex changes and maintain sustainable routines. The authors argue that the same feedback system should support those stages in parallel. Furthermore, studies have shown that the even though eco-feedback systems promote awareness to consumers, they are more impactful when used by already pro-environment motivated individuals (Wallenborn et al., 2011). The same observation was made by McCalley and Midden (McCalley and Midden, 2002), where one hundred participants performed a simulated task (operating a washing machine). The research team observed that participants with consumption goals (self-set and assigned) spent less energy than the control group. Additionally, the authors also noted that pro-self-individuals saved more energy when allowed to choose their own goals, and prosocial participants saved more energy when they were assigned goals. Researchers have identified four distinct motivations for the adoption of ecofeedback systems: financial (reducing the energy bill, saving money); environmental (reducing consumer impact on the environment); information gathering (real-time detailed usage) and technological (a tool to help household members in their routines) (Chetty et al., 2008).

Next in this sub-chapter we describe specific frameworks and models used in behaviour change. Next we present thoroughly three strategies and concrete studies that employed those approaches to motivate individuals to reduce energy consumption, all these three strategies were used in specific studies throughout this thesis.

\subsubsection{Frameworks}

Fogg's Behavior Model for Persuasive design (Fogg, 2009) has been used to, an extent, as framework for behaviour change in eco-feedback devices, both in the design and analysis. Fogg's models the conditions for an individual to change its behaviour in three separate topics: i) The person should be sufficiently motivated; ii) Have the ability to perform the behaviour; iii) Be triggered to perform the behaviour. Fogg also notes that these three factors must occur simultaneously for behaviour change to happen. 
Another psychology model used in eco-feedback interventions is the Transtheoretical Model (TTM). The model itself was created to understand individuals' willingness to act on healthier behaviors, it encompasses five steps of change: Precontemplation, Contemplation, Preparation, Action and Maintenance. He et al. (He et al., 2010) reviewed a substantial amount of sustainability/eco-feedback work and discussed thoroughly how TTM has been applied in those studies. The authors present recommendations of how an eco-feedback system could assist in any of the abovementioned stages. The recommendations and rationale behind them are based on an extensive motivation psychology review.

In a more concrete level, several other strategies have been used to motivate individuals in eco-feedback studies. The Information Model (Wright and Kacmar, 1994) considers that simply providing information will motivate individuals to change their behaviour relatively to a problem (assuming that individuals' understand that a problem exists). For example, presenting consumption information. Positive reinforcement (Hockenbury and Hockenbury, 2012) is a technique which consists of adding a positive stimulus after a positive action towards the targeted behaviour. According to positive reinforcement, this will increase the probability of that action happening again. The Elaboration Likelihood Model (Petty et al., 2005) proposes two routes to persuasion: The central route persuasion will occur from individuals' careful consideration of the information presented, the resulting behaviour will likely be long lasting. The peripheral route is not so much affected by logic, it results from emotional persuasion which might not be directly in line with the information validity. The Attitude Model (Costanzo et al., 1986) assumes that "attitudes cause behaviours". For example, a person with a positive attitude towards the environment will more likely behave in a way to conserve it. This technique has been used by government and utilities companies with campaigns aimed at empathizing consumers with the environment. The Rational-Economic-Model (Wright and Kacmar, 1994) assumes that the monetary cost the first incentive for a pro-environmental behaviour. In the light of this model simply presenting the cost of the energy being consumed with be a strong motivator to reduce individual consumption.

\subsubsection{Social incentive to conserve}

Another area of research pertaining to eco-feedback is what has been called socially enabled eco-feedback (Foster et al., 2010). These systems take advantage of peer pressure and competition as motivators to reduce resource consumption. This type of system is often deployed as public displays that show collective behaviour, or make comparisons between the individual and the others (e.g. (Jönsson et al., 2010; Moere et al., 2011; Pierce et al., 2008)). 
More recently social networks have also been widely used in similar studies (e.g (Filonik et al., 2013; Mankoff et al., 2010; Petkov et al., 2011)). In the rest of this section we present a series of studies that have used peer pressure and competition with the end goal of reducing energy consumption. Considering our work, the WattsBurning in my mailbox study was highly motivated by the studies presented below, additionally our work also contributes to this field, more on that particular study in section 4.6.

In a study conducted at the Oberlin College (Petersen et al., 2007), researchers organized a dormitory energy consumption competition in which residents received near real-time and weekly feedback about their consumption. This study produced encouraging results. The 16 buildings receiving weekly feedback reduced their consumption by $31 \%$ during the study while the two buildings receiving real-time data decreased their consumption by $55 \%$. Building on these results the authors concluded that real time information is more effective than weekly information because it allows the residents to adapt and learn more about the impact of their habits and appliances (Petersen et al., 2007). To understand the same motivation techniques a similar competition (Odom et al., 2008) was conducted at Indiana University but combining water and electricity consumption. A public ranking was updated twice a week and displayed the water and electricity consumption by dormitory as a percentage against a one-month baseline. The study lasted one month and the participants saved $33008 \mathrm{kWh}$ of electricity and 724 gallons of water when compared to a baseline of the three previous years, participants reported that they were influenced by their peers, and the research team agreed that social motivation was important for the success of the competition. The authors also share some insights for displaying information on eco-feedback devices. For example, while the authors argue that numerical representation of personal consumption is suitable to encourage behavioural change, they also argue that the same numerical information representing aggregate consumption (e.g. consumption by neighbourhood or building) can discourage users from changing their behaviour.

Peschiera et al (Peschiera et al., 2010) also performed a study in a dormitory building, in which 83 rooms were installed with energy monitors. The rooms were divided in three groups (besides a control group). The first one received information about their consumption, the second group received information about their consumption contextualized with the average consumption of the building and finally the third group was informed of their own electricity use contextualized with both average occupant utilization and the electricity of a peer network of occupants in the building. The only group which consumption was significantly different from the control group was the third one. 
The promising results of these studies drove researchers to include social aspects of motivation into personal energy meters (Foster et al., 2010), together with other consumptionreduction techniques (Filonik et al., 2013). Foster et al developed the Wattsup (Foster et al., 2010) which is a system developed to work with the Wattson energy monitor. The system was designed for a focus group of four users. Later a study was conducted in eight homes over a period of 18 days. The authors used two conditions during the study, in one of them participants were able to consult only their information whereas in the second one they could access the consumption of other participants as well as their own. This second condition triggered significant reduction in energy consumption when compared to the first group. Interviews revealed that the competition between participants motivated them to conserve energy. The authors did not refer to any privacy concerns by the users. There were a number of interesting suggestions from the users - for example a digital photo frame that would change its photo according to the household energy consumption.

Moere et al. (Moere et al., 2011) combined a public display of the household information with a commercial energy meter deployed in each participant's house. The study lasted seven weeks comprising 12 households. The electricity consumption of each house was displayed on a blackboard on the façade of the individual houses (Figure 7 left). The display showed personal messages for each household. Daily performance was presented as a percentage of savings compared with the previous day, the ranking against the rest of the neighbourhood and an historical graph of the consumption. Additionally, each family also received one commercial Efergy electricity monitor, which was also responsible for sending the data to the research team to update the displays. After the seven weeks, families with the public display had reduced their consumption by $2.5 \%$ per week. Families without the public display but with the in-house monitor had saved 1\% per week. Families with no feedback had reduced their consumption by $0.5 \%$. A similar study is proposed in (Bird and Rogers, 2010) where the authors describe a framework and a deployment to show consumption for the inhabitants of Tidy Street a residential road in the Center of Brighton, United Kingdom. The main goals of the research team were to build a low-tech energy eco-feedback system to measure a household's energy use, and display the information in an effective way. The research team planned to show the consumption in three different ways: individually for each household, publicly on Tidy Street, and finally over the internet. In the end the research team opted for a lower-tech solution where individual consumption for each household was displayed with chalk spray on large-scale visualizations on the streets. The visualization compared how the Tidy Street average consumption compared with the Brighton average consumption. After three weeks the average consumption of the street was reduced by $15 \%$. 
The rapid development of virtual social networks, and the increasing availability and capability of these platforms, provided researchers with a large and easily accessible research test opportunity for motivation techniques that take advantage of social interactions. For example Flonik et al. (Filonik et al., 2013) presents the Dashboard display - a study of mobile energy-monitoring tools. The application provided several widgets and features related to sustainability and social networks (for example charts showing the consumption of electricity, Facebook news feeds or weather forecasts), and it was deployed with 13 participants for five weeks. The application was well-received, but it was observed that the element that kept participants connected to the application was the ability to share and compare energy consumption. Participants who shared their energy consumption were more likely to exchange knowledge and expertise about sustainability. StepGreen (Mankoff et al., 2010) is a "green" social network, that allows members to publicly commit to performing certain sustainability actions (Figure 5 middle). The website also supported discussion between members. While using Stepgreen almost every user mentioned that the social network allowed them to learn something about an easy-to-perform action with a positive impact on the environment. In a posttest survey $45 \%$ of the participants reported that using the social network made them more aware of the impact of household energy consumption on global warming. It was also reported that the projected and current savings displayed on the website were appreciated by participants.

Public displays can also be used with different goals apart from competition, for example to promote awareness and discussion. The Nuage Vert described in ("Reading the clouds," 2008) was an art installation in Helsinki, on which the city energy consumption was displayed with lasers projected onto the smoke of a coal energy power plant. For seven days the smoke cloud was projected with green colours (the projection was actually the outline of a cloud), the lower the consumption the larger the illumination. On the final day residents were asked to unplug the devices to reduce consumption, to increase the size of the green cloud. This resulted in a reduction of the peak demand by $800 \mathrm{kVA}$.

A similar study was performed by Jönsson et al (Jönsson et al., 2010), which consisted of a public display of electricity consumption made with three circular projections in the floor. The projections represented the maximum, minimum and real-time consumption respectively (Figure 7 Right). This installation was deployed in eight industries in Sweden and the research team observed that when placed in public places the installation promoted discussion between employees and some of them even made annotations next to the projections. 

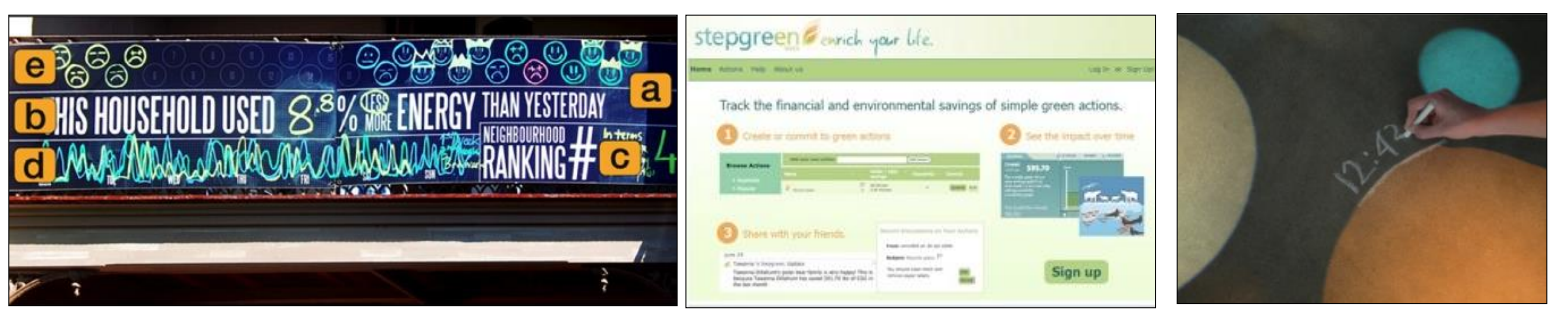

Figure 7: Left; Public display used in (Moere et al., 2011); Middle: StepGreen Social network (Mankoff et al., 2010b); Right: Projections used in the WattLite prototype (Jönsson et al., 2010).

\subsubsection{Displaying the impacts of energy consumption in the environment}

An alternative paradigm for feedback devices is to inform consumers of the impact of their actions on the environment. Commercial feedback systems have tried to present this information as pounds of $\mathrm{CO} 2$ or number of threes required to offset the emission values (i.e. the amount of threes necessary to absorb the emitted CO2). Lately, artists and designers have also engaged in the sustainability challenge, in particular through the use of technology and aesthetically rich interventions in which the information is presented with visual metaphors or animations of the impact on the environment. The motivation behind those strategies is that the 5 to $10 \%$ saving promoted by eco-feedback devices might not be enough financial motivation for individuals to reduce electricity consumption. Therefore, researchers employ different techniques that appeal on the connection between persons and the environment, next we present a series of studies that have deployed these interaction and visualization strategies in the sustainability/eco-feedback field. Work in this field influenced greatly a paradigm shift in our work (section 4.4) in which we developed eco-feedback devices where the household consumption was mapped as elements of the local forest, this way, this is also a field in which our work contributes.

Tiffany Holmes in 7000 Oaks and Counting (Holmes, 2007) for example, uses an animation of a set of 3 images to show an estimate of the number of threes needed to offset the $\mathrm{CO} 2$ generated during the production of electricity consumed in a specific building (Figure 8 left). The Stepgreen system uses a set of animals (seals, polar bears and fishes) near an iceberg - as the iceberg melts the animals disappear depending on the individual sustainable actions, the system was deployed for 3 weeks with 32 individuals that used the social network, which completed a questionnaire before the study and interviews after the study. Nuage Vert ("Reading the clouds," 2008) also mentioned in section 3.5.2, also aimed to raise awareness by enhancing the smoke cloud resultant from the thermal station which feeds the city electricity. 
Froehlich et al. (J. Froehlich et al., 2009a) designed Ubigreen (Figure 8 right) a mobile application designed to promote greener transportation habits. The application displays two different types of iconic feedback as the background picture on the phone. A tree appears with more leaves, flowers or fruit depending on the amount of green transportation made by a participant. In the other animation a polar bear is shown on an iceberg, the iceberg will grow and other animals will appear based on user performance. After a 3-week field study with 14 participants in which interviews and transit data were analysed, the study reports that having the visual feedback as the background of the phone made them more aware and motivated them to reflect on their transportation habits. Curiously some participants disclosed that negative reinforcement would have been valuable addition to the design of the system.

The Power-Aware Cord designed at the Static! Institute (Gustafsson and Gyllenswärd, 2005 ) is the redesign of a common power strip that displays a visualization of the electricity passing through it. The author argues that the intangible/invisible nature of electricity makes consumers take it for granted. With the approach of showing the electricity flowing through the cord the authors have aimed at user playfulness as a trigger for exploration of the different electrical devices. The prototype was tested with 15 people, and after a qualitative evaluation the authors report that most participants quickly grasped the concept of the power-aware cord. The same institute installed batteries, LEDs and optical fibre in a household curtain creating the Energy Curtain (Ernevi et al., 2005). The curtain collects energy from the sun during the day and then it lights the room when the sun goes down. The user has then to decide between lighting the room with sunlight during the day or collecting energy to be used at night. The Erratic Radio was also designed within the Static! Institute (Backlund et al., 2006). This functions like a normal radio which besides receiving normal radio frequencies also detects frequencies in the $50 \mathrm{~Hz}$ range (normally emitted by electric appliances). This means that in order to listen to the radio, consumers have to balance the energy consumption of appliances next to the radio or else the signal quality will decrease as the consumption increases (Figure 8 middle).

Rodgers and Bartram (Rodgers and Bartram, 2011) exposed participants to three different abstract visual forms of ambient display for electricity consumption. Participants had to perform two different tasks (between subject design) i.e. operate a home entertainment system and make a snack in a kitchen. Both the kitchen and the home entertainment system were set-up in the lab, the evaluation method included questionnaires, observer notes, attention logs, energy use logs, and semi-structured in-depth interviews. Sixteen of the 23 participants correctly interpreted the feedback. The research argues that the representation of consumption must be clear to avoid confusion, and even though some ambiguity in the visualization can encourage curiosity, it can 
also make user comprehension difficult. Furthermore, abstract representation can also be displayed with concrete numerical data, this way the ambient display would be another technique in a more complete eco-feedback system.

In summary, research has shown that dramatic, sensational, fearful and shocking representation for the effects of energy consumption in the environment (e.g. climate change) are useful to capture people's attention to sustainability issue, however these strategies should be carefully used since they may induce feeling of helpless and overwhelm in individuals when they try to relate their daily actions to the global impact, (O'Neill and Nicholson-Cole, 2009) therefore these strategies are useful as initial hooks to individuals attention to sustainability issues, however after that initial period consumers should get accurate and actionable information in how to reduce their impact on the environment.
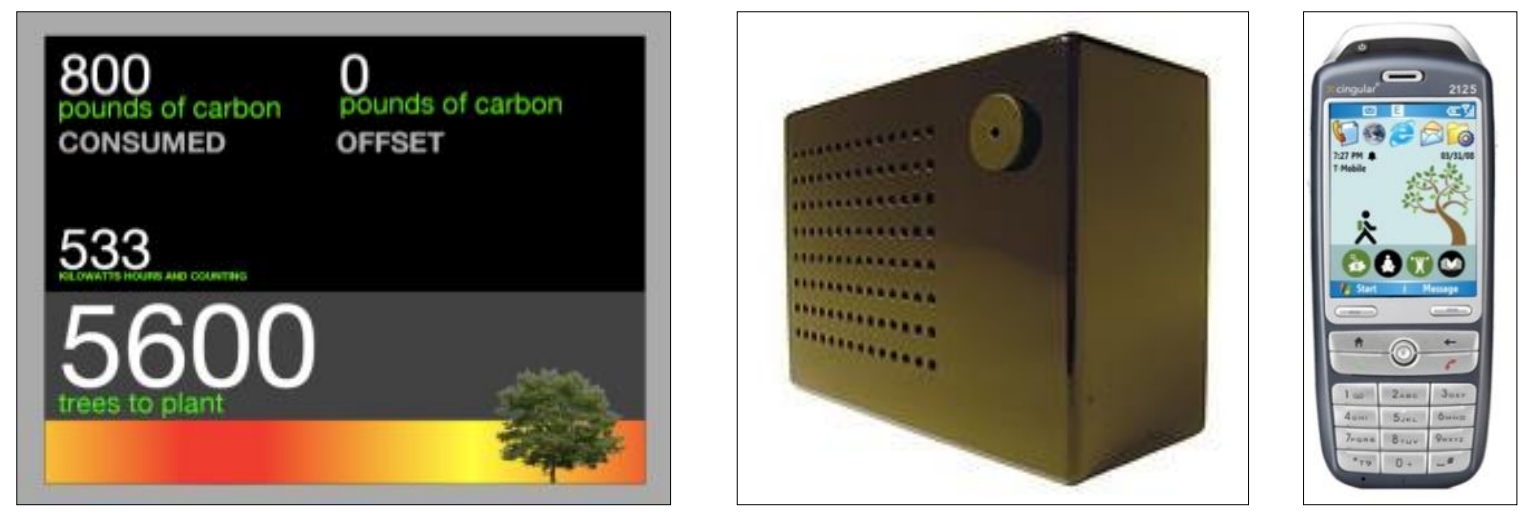

Figure 8: Left: 7000 oaks and counting system (Holmes, 2007a); Middle: Erratic radio developed by the

Static! Institute (Ernevi et al., 2009). Right: Ubigreen mobile application (J. Froehlich et al., 2009).

\subsubsection{Energy production feedback}

A different approach to connecting consumption to the effects on the environment is to present consumers with the source of their electricity. Even though real production data is hard to come by, this concept was tested with micro-generation scenarios reporting increased awareness and knowledge of electricity consumption (Filonik et al., 2013; Pierce and Paulos, 2012b). Next we present a set of studies in which the source of electricity was a variable in the feedback or was in some form incorporated into the analysis of the results. Feedback from these studies influenced significantly the last steps of our work, in which we aimed at presenting consumers with accurate impact on the environment of their behaviours. 
In (Woodruff et al., 2008) the authors observed that users that had solar panels installed in their homes, followed their production closely, monitoring the weather to better understand the production and consumption. The close relationship between consumption and production helped participants tune their routines, and presented them with concrete data of their contributions to the environment, as well as helping households to decrease their utility bills. A similar observation was made by Filonik et al (Filonik et al., 2013) in which the authors designed and deployed a dashboard display with information about energy consumption and sustainability (Figure 9 Right). The research team observed that participants with solar panels used a weather widget to predict and better understand the production values of their households.

Also in the micro-generation field Pierce et al (Pierce and Paulos, 2012b) developed the Local Energy Indicator prototype (Figure 9 Left) which uses the idea of energy metadata. It provides information about the source and location of the consumed energy. The authors argue that micro-generation systems could be designed to be desirable not only by helping consumers save money but also by appealing to user satisfaction. The same authors (Pierce and Paulos, 2010) argue that because of the intangible nature of electricity consumers are largely unaware of the material production of energy and, in many ways, the actual consumption of this energy. The author designed and evaluated several prototypes that were capable of representing the intangible quality of the energy. One of these prototypes was the Local Energy Lamp that displayed energy metadata such as source and age. Participants referred to the fact that they could change their routines in relation to the availability of solar or wind energy. Finally the authors argue that services should be designed to take advantage of the observed engagement between consumers and solar energy. Similarly, Wallenborn et al (Wallenborn et al., 2011)conducted a study with an energy meter in 21 households. The authors conclude by arguing that in order to empower all electricity consumer research should work to make energy more "precious", since the energy monitor only truly developed awareness in informed consumers.

The importance of creating a connection between individuals and the source of their energy was further reinforced by Chetty et al. (Chetty et al., 2008), by performing a qualitative study of 15 households about their practices regarding water, electricity and natural gas systems at home. Some participants that lived in a sub-divided house with a shared utility infrastructure had more constrained choices for buying "green" energy (energy produced using environmentally friendly methods such as solar, wind or water power) because of having to negotiate this with their neighbours. Furthermore, the authors aimed at observing common practices around the house related to resource consumption, and how these could be improved 
by technology. One of the design guidelines for Ubiquitous Computing (Ubicomp) systems is, for example, to make in-the-moment resource consumption visible in terms that make the costs of production and units of consumption more obvious.

Connecting electricity production with consumption and with the goal of fostering greater knowledge and engagement in consumers can result in consumers changing their routines to consume more in periods where there is higher availability of renewable energy (Hledik, 2009). This phenomenon is commonly called peak demand shifting, and could result in a reduction in emissions that have the potential to be bigger than the values promoted by eco-feedback devices (5\% 15\%) since production can be shifted to periods where more renewables are available or to periods where the thermal plants work with higher efficiency. Therefore apart from the personal goal of feeling good about yourself, this shift is also valued by the electricity provider since it often results in higher profits when, for example, expensive-to-operate generators are disabled.

Kjeldskov et al. build the eFORECAST a system that displayed recent usage, expected usage and price of electricity (Kjeldskov et al., 2015), additionally the system also displayed the availability of wind power and expected peaks in demand. The system was tested for approximately six months in three households, and the authors observe that in general consumers "like" the idea of forecasting the availability of the electricity, furthermore the authors also claim that households' were expecting a bigger financial reward for the effort of shifting the domestic electricity use, which by itself is a challenging. Similarly, Simm and colleagues engaged remote islanders in as equal partners in a research project that investigated the topic of synchronizing energy consumption with supply, and together built a prototype renewable energy forecast display. In their study, despite having no financial incentive, a number of participants described a change in their practices, saving high-energy tasks for times when local renewable energy was expected to be available (Simm et al., 2015).

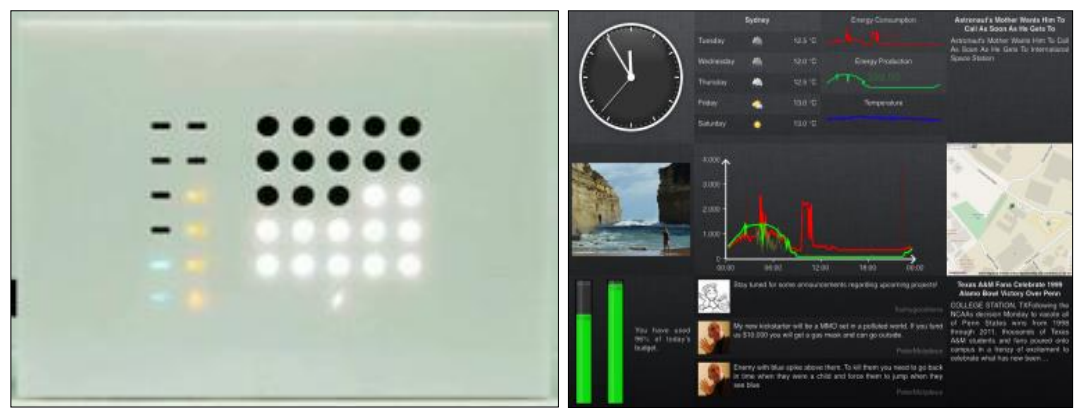

Figure 9: Left: The local energy indicator (Pierce and Paulos, 2012); Right: Visualization used in (Filonik et al., 2013). 


\subsection{Effectiveness of eco-feedback over time}

Even though the majority of short-term research studies support the fact that eco-feedback systems result in energy savings, long-term studies have shown that people tend to ignore ecofeedback systems over time. This does not necessarily mean that users will return to their behaviour prior to the introduction of the feedback system, but this negative implication was found by (Holmes, 2007; Peschiera et al., 2010). Some authors argue that the cause of this phenomenon is that users become more aware of their consumption and change their behaviour regardless of the eco-feedback system. These authors claim that eco-feedback technology would be more effective if it evolved over time along the same path as the user in order to keep pace with their deepening commitment and understanding (Woodruff et al., 2008)

The same observation was made by Peschiera in a study where an 83 room residential dormitory was using energy monitors. The research team observed that after a period of usage of feedback the electricity consumption relapsed to values prior to the study (Peschiera et al., 2010). Holmes reported this phenomenon in (Holmes, 2007) where it was possible to observe users gradually returning to their previous behaviours as feedback became less frequent or was no longer present.

Similarly, Yang et al. (Yang et al., 2014) in a study aimed at assessing households' usage of thermostat devices. The study evaluated several types of thermostats, among them 19 houses used the Nest thermostat. That although is not an eco-feedback device per-se, it contains and advertises several energy savings features. The authors claim that "the engagement with the Nest, along with the frequency of interaction, diminished and users' interactions settled into patterns that resulted in missed opportunities for energy savings".

The effectiveness of eco-feedback over time is one of the central themes of this thesis, therefore the work presented above was already considered in the introductory section of this thesis, (section 2.1) and it will also be taken into account in the evaluation of our practical studies. 


\subsection{A Survey of Energy Monitoring: Summary}

The following table presents a summary of all studies in the reviewed work. In this summary we only considered work that was deployed "into the wild" and used by regular people. This decision excludes lab tests used to analyse single feedback techniques and implementations.

The presented studies are described by the kind of sensing technology used (e.g. NILM, self-report), the hardware/software modality used in the feedback (e.g. mobile phone, website), number of participants that participated in the study, its duration and a summary of the results.

Table 2: Summary of eco-feedback studies.

\begin{tabular}{|c|c|c|c|c|c|}
\hline Publication & $\begin{array}{l}\text { Sensing } \\
\text { technology }\end{array}$ & Feedback & $\begin{array}{l}\text { Number of } \\
\text { participants }\end{array}$ & Duration & Results summary \\
\hline (Abrahamse et al., 2007) & $\begin{array}{l}\text { Gas and Water } \\
\text { meters }\end{array}$ & $\begin{array}{l}\text { Cusom made } \\
\text { Website with } \\
\text { tailored } \\
\text { feedback }\end{array}$ & 189 indivdiuals & 5 months & $\begin{array}{l}\text { After a period of } 5 \text { months, a combination of } \\
\text { tailored information, goal setting and } \\
\text { feedback was especially successful in } \\
\text { reducing gas, electricity and fuel use (i.e. } \\
\text { direct energy use). By customizing } \\
\text { interventions to specific characteristics of } \\
\text { target groups the effectiveness of } \\
\text { interventions may be enhanced }\end{array}$ \\
\hline
\end{tabular}




\begin{tabular}{|c|c|c|c|c|c|}
\hline (Broms et al., 2010) & Smart meter & $\begin{array}{l}\text { Custom made } \\
\text { software/hard } \\
\text { ware feedback } \\
\text { system }\end{array}$ & 9 houses & 3 months & $\begin{array}{l}\text { The central placement and visibility } \\
\text { sometimes became a bit of a provocation in } \\
\text { themselves preventing some households } \\
\text { from fully accepting the meter } \\
\text { Patterns on the clock allowed the } \\
\text { identification of single appliances from the } \\
\text { total load chart }\end{array}$ \\
\hline (Chetty et al., 2008) & No feedback & No feedback & 33 individuals & $2 / 3$ months & $\begin{array}{l}\text { "modify their homes for resource efficiency } \\
\text { for saving money, maintaining a comfortable } \\
\text { setting and to a lesser extent, to be } \\
\text { environmentally friendly. Specifically, } \\
\text { householders desire insight into the impact of } \\
\text { their changes to their homes and behaviors on } \\
\text { resource usage and more visible real-time } \\
\text { information on within the home resource } \\
\text { consumption" }\end{array}$ \\
\hline (Erickson et al., 2013) & Smart meters & Website & 18 individuals & 20 weeks & $\begin{array}{l}\text { Decrease in energy used and understanding } \\
\text { of electricity by selected households }\end{array}$ \\
\hline
\end{tabular}




\begin{tabular}{|c|c|c|c|c|c|}
\hline & & & & & $\begin{array}{l}\text { The most likely instigator for changes are } \\
\text { crisis or price in the cost of energy. }\end{array}$ \\
\hline (Filonik et al., 2013) & $\begin{array}{l}\text { Different sensors } \\
\text { and streams of } \\
\text { data }\end{array}$ & $\begin{array}{l}\text { Native } \quad \mathrm{PC} \\
\text { application }\end{array}$ & 12 participants & 5 weeks & $\begin{array}{l}\text { Customization was appreciated by users, they } \\
\text { were enthusiastic about composition of } \\
\text { widgets that allowed them to investigate } \\
\text { relationships between the data - for example } \\
\text { between weather and energy consumption. } \\
\text { Participants that shared their energy } \\
\text { consumption with other were likely to } \\
\text { exchange expertise and troubleshoot } \\
\text { problems. }\end{array}$ \\
\hline (Foster et al., 2010) & $\begin{array}{l}\text { Commercial } \\
\text { whole house } \\
\text { sensing system }\end{array}$ & $\begin{array}{l}\text { Facebook } \\
\text { application }\end{array}$ & 8 houses & 18 days & $\begin{array}{l}\text { Social network sites might be very important } \\
\text { in the future of energy consumption in the } \\
\text { home }\end{array}$ \\
\hline (J. Froehlich et al., 2009a) & $\begin{array}{l}\text { Sensors and self- } \\
\text { report used to } \\
\text { monitor }\end{array}$ & $\begin{array}{l}\text { Mobile phone } \\
\text { application }\end{array}$ & 13 participants & 3 weeks & $\begin{array}{l}\text { Participants liked the application and showed } \\
\text { interest in the information presented by it }\end{array}$ \\
\hline
\end{tabular}




\begin{tabular}{|c|c|c|c|c|c|}
\hline & $\begin{array}{l}\text { transportation } \\
\text { activities }\end{array}$ & & & & \\
\hline (Gamberini et al., 2012a) & $\begin{array}{l}\text { Wireless sensors } \\
\text { in each power } \\
\text { socket }\end{array}$ & $\begin{array}{l}\text { Custom-made } \\
\text { application } \\
\text { interface }\end{array}$ & 4 houses & 4 months & $\begin{array}{l}\text { Smart advices were well received and } \\
\text { supported statistically by a significant drop in } \\
\text { consumption (after each smart advice) that } \\
\text { reached } 38 \% \text { in specific instances }\end{array}$ \\
\hline (Darby, 2001) & $\begin{array}{l}\text { Smart energy } \\
\text { monitors }\end{array}$ & $\begin{array}{l}\text { Smart energy } \\
\text { monitors } \\
\text { feedback }\end{array}$ & $\begin{array}{l}15 \text { households } \\
\text { (from a bigger } \\
\text { sample) }\end{array}$ & $\begin{array}{l}\text { Between } 1 \\
\text { and } 2 \text { months }\end{array}$ & $\begin{array}{l}\text { The monitors are domesticated into the } \\
\text { physical domain, social relations and cultural } \\
\text { practices of each household. }\end{array}$ \\
\hline
\end{tabular}




\begin{tabular}{|c|c|c|c|c|c|}
\hline & & & & & $\begin{array}{l}\text { Context is fundamental to understanding the } \\
\text { extent to which change effects will be } \\
\text { negotiated and realised }\end{array}$ \\
\hline $\begin{array}{l}\text { (Jakobi and Schwartz, } \\
\text { 2012) }\end{array}$ & $\begin{array}{l}\text { Smart meters }+ \\
\text { smart plugs }\end{array}$ & $\begin{array}{l}\text { Home energy } \\
\text { monitoring } \\
\text { system }\end{array}$ & 9 houses & $\begin{array}{l}\text { Between } 1 \\
\text { and } 3 \text { months }\end{array}$ & $\begin{array}{l}\text { The contextualization of data is crucial in } \\
\text { enabling the user to apply individual energy } \\
\text { practices when judging the consumption }\end{array}$ \\
\hline (James Pierce, 2010) & $\begin{array}{l}\text { Commercial plug } \\
\text { level }+ \text { whole } \\
\text { house meters }\end{array}$ & $\begin{array}{l}\text { Commercial } \\
\text { meter } \\
\text { interface }\end{array}$ & 9 participants & 3 to 12 days & $\begin{array}{l}\text { Feedback devices might encourage users to } \\
\text { maintain a baseline consumption instead of } \\
\text { decreasing it Many of the energy-related } \\
\text { interactions are non-negotiable }\end{array}$ \\
\hline (Kjeldskov et al., 2012b) & Smart meters & $\begin{array}{l}\text { Mobile } \\
\text { application }\end{array}$ & 10 houses & 7 weeks & $\begin{array}{l}\text { Different kinds of views on the system could } \\
\text { assist understanding the energy consumption. } \\
\text { The mobile nature of the feedback was used } \\
\text { to access data away from home }\end{array}$ \\
\hline
\end{tabular}




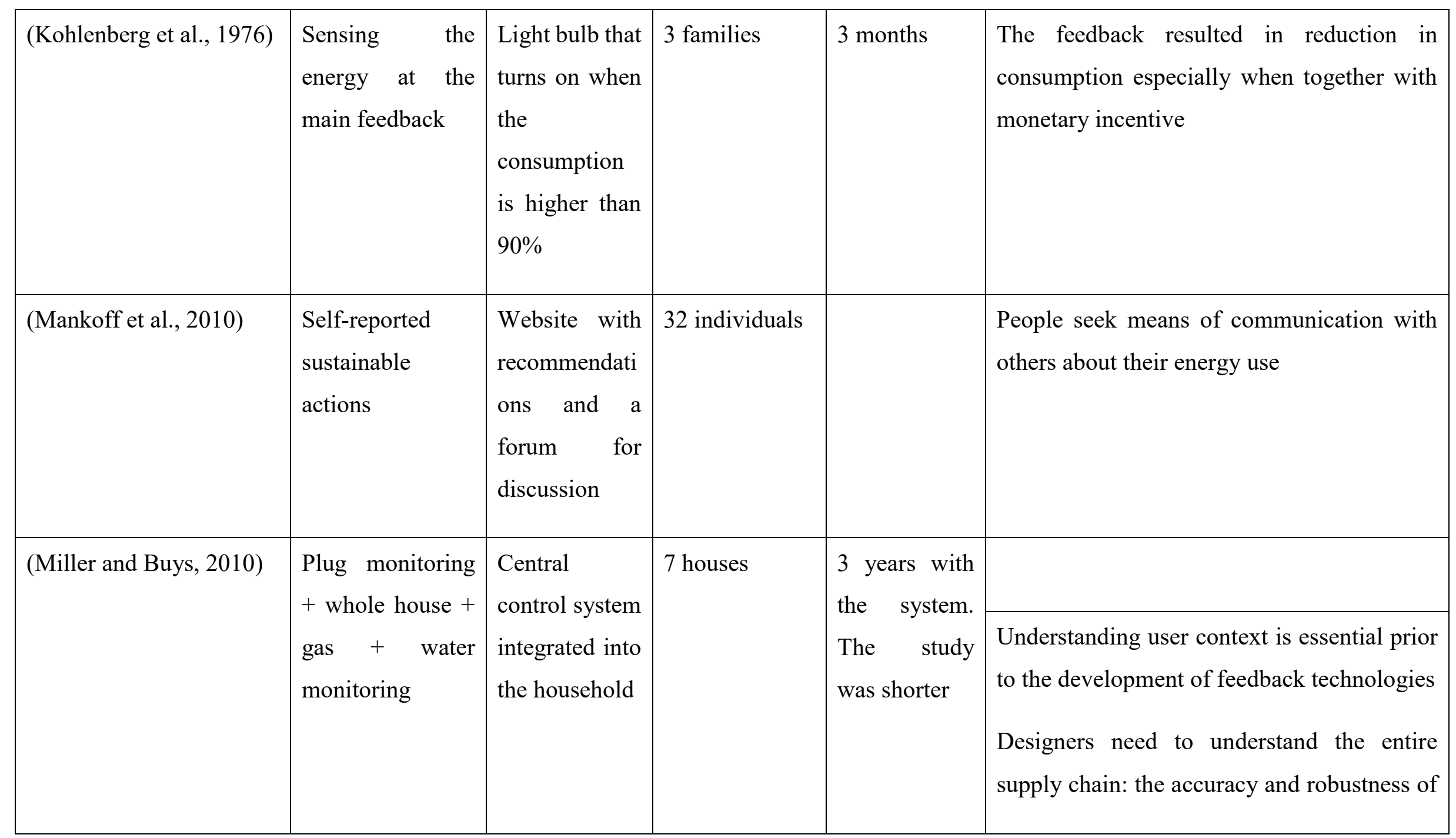




\begin{tabular}{|c|c|c|c|c|c|}
\hline & & & & & $\begin{array}{l}\text { the meters and sensors, the installation ease, } \\
\text { and interconnection reliability }\end{array}$ \\
\hline (Moere et al., 2011) & $\begin{array}{l}\text { Commercial } \\
\text { system }\end{array}$ & $\begin{array}{l}\text { Public display } \\
\text { outside the } \\
\text { house }+ \text { in } \\
\text { house } \\
\text { commercial } \\
\text { meter } \\
\text { feedback }\end{array}$ & 11 participants & 7 weeks & $\begin{array}{l}\text { houses with public display reduced the } \\
\text { energy usage more than the control group" } \\
\text { the single numerical ranking of participants } \\
\text { played too much of an overwhelming role in } \\
\text { motivating people into short term } \\
\text { competitive behavior }\end{array}$ \\
\hline (Odom et al., 2008) & N.A. & Website & 10 dormitories & 28 days & $\begin{array}{l}\text { The dorm that won the competition was able } \\
\text { to save } 33.008 \mathrm{kWh} \text { and } 723322 \text { gallons of } \\
\text { water against the baseline }\end{array}$ \\
\hline (Patel et al., 2007) & $\begin{array}{l}\text { High frequency } \\
\text { sampling of the } \\
\text { noise each } \\
\text { appliance }\end{array}$ & $\begin{array}{l}\text { No feedback } \\
\text { just testing the } \\
\text { algorithm }\end{array}$ & 5 homes & $\begin{array}{l}1 \text { week to test } \\
\text { the algorithm }\end{array}$ & $\begin{array}{l}\text { The study indicated that the proposed } \\
\text { algorithm was able to identify electrical } \\
\text { events with } 8590 \% \text { accuracy }\end{array}$ \\
\hline
\end{tabular}




\begin{tabular}{|c|c|c|c|c|c|}
\hline & $\begin{array}{l}\text { introduces in the } \\
\text { house grid. }\end{array}$ & & & & \\
\hline
\end{tabular}




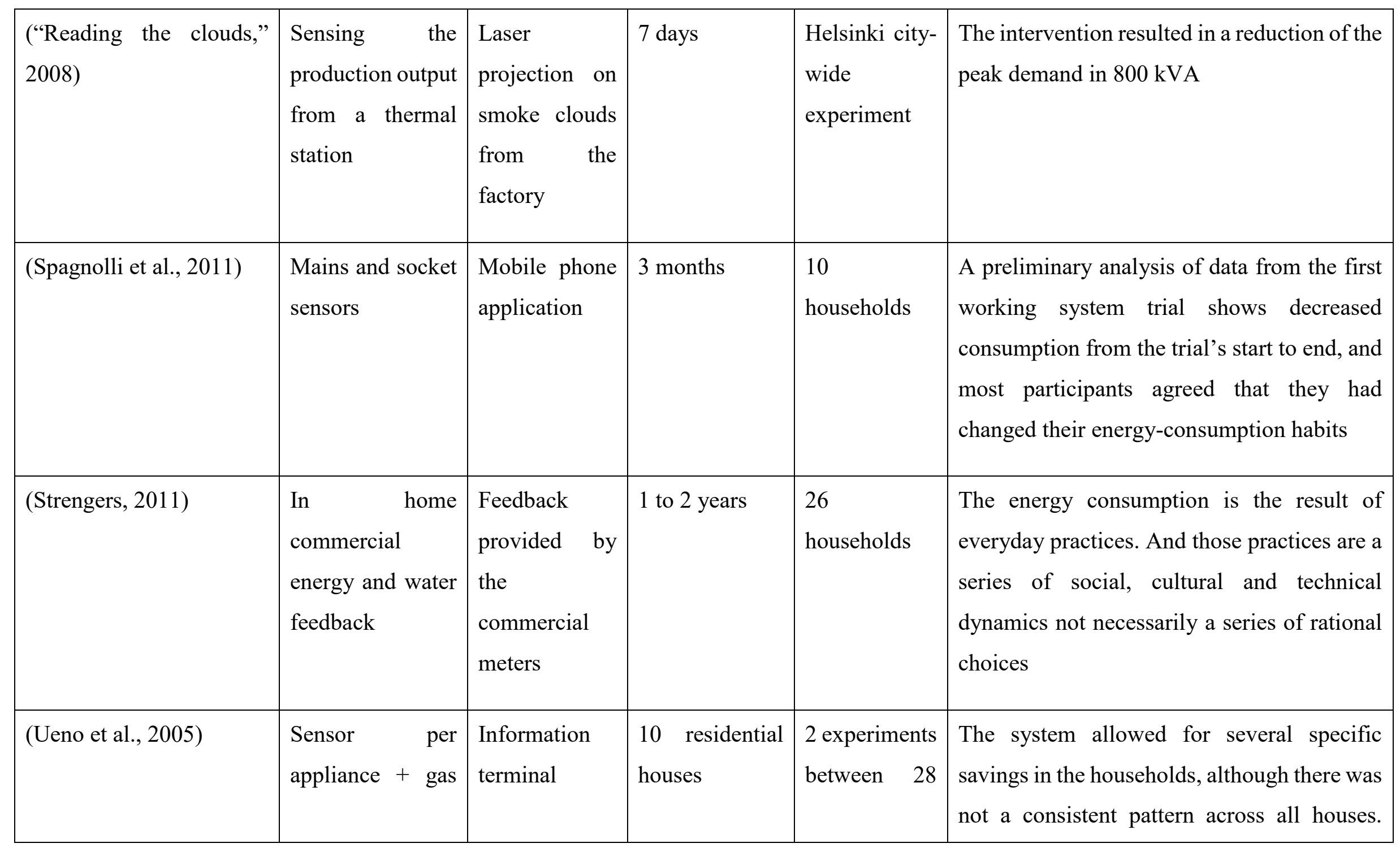




\begin{tabular}{|c|c|c|c|c|c|}
\hline & $\begin{array}{l}\text { measured every } \\
30 \text { minutes }\end{array}$ & $\begin{array}{l}\text { installed in a } \\
\text { netBook }\end{array}$ & & $\begin{array}{l}\text { and } 40 \\
\text { weekdays }\end{array}$ & $\begin{array}{l}\text { However during the study the there was a } \\
\text { decrease of } 18 \% \text { of gas and } 9 \% \text { of electricity } \\
\text { consumption }\end{array}$ \\
\hline (Wallenborn et al., 2011) & $\begin{array}{l}\text { Commercial } \\
\text { meters }\end{array}$ & $\begin{array}{l}\text { Feedback } \\
\text { given by the } \\
\text { commercial } \\
\text { metres }\end{array}$ & 21 houses & 2 to 4 weeks & $\begin{array}{l}\text { All households learned something } \\
\text { Difficult to interpret the kWh metric } \\
\text { Savings expressed in euros did not impressed } \\
\text { users }\end{array}$ \\
\hline (Woodruff et al., 2008) & $\begin{array}{l}\text { Different systems } \\
\text { bought by home } \\
\text { owners }\end{array}$ & $\begin{array}{l}\text { Different } \\
\text { types of } \\
\text { feedback } \\
\text { depending on } \\
\text { the family }\end{array}$ & 35 houses & n.a. & $\begin{array}{l}\text { The authors claim that eco-feedback } \\
\text { technology would be more effective if it } \\
\text { evolved over time along the same path as the } \\
\text { user in order to keep pace with their } \\
\text { deepening commitment and understanding. } \\
\text { Followed their production closely, } \\
\text { monitoring the weather to better understand } \\
\text { the production and consumption }\end{array}$ \\
\hline
\end{tabular}




\begin{tabular}{|c|c|c|c|c|c|}
\hline (Yun, 2009) & $\begin{array}{l}\text { Arduino } \\
\text { microprocessor }+ \\
\text { CT clamp }\end{array}$ & $\begin{array}{l}\text { Custom made } \\
\text { stationary and } \\
\text { portable } \\
\text { feedback } \\
\text { devices }\end{array}$ & 8 houses & 3 weeks & $\begin{array}{l}\text { Minimalist real-time feedback was effective } \\
\text { at increasing awareness of low and moderate } \\
\text { users. But some wanted more information. } \\
\text { An eco-feedback device should provide both } \\
\text { modes of information }\end{array}$ \\
\hline
\end{tabular}

The table above, draws a transparent picture of current eco-feedback research, which is characterized by the diversity of approaches (in-house case studies, public displays) and technologies used (commercial meters, custom made frameworks), to achieve the end goal of reducing the environmental impact. Our research builds on the presented work in all of the categories above, but mostly our work presents a series of eco-feedback studies performed in the wild, in which the finding from one are incorporated in the next one. 


\section{Research Studies}

In this chapter we present the practical research work carried out throughout this thesis. Firstly, we describe the deployments where our research in the wild was performed (section 4.1). Secondly, we describe the underlying hardware research platform used in our eco-feedback studies (section 4.2). In the third section of this chapter we present the initial iterations of deployment of our eco-feedback device (section 4.3). Finally, in the last subsections we describe each deployment study, the motivation behind it, its evaluation and results (sections 4.4, 4.5, 4.6, 4.7 and 4.8).

\subsection{Deployments Setting}

All the deployments performed for this thesis work, took place in Madeira Island. Madeira is located in the North Atlantic Ocean, approximately 1000km from mainland Europe, and 520km from the African coast. The island has an area of approximately $801 \mathrm{~km}^{2}$ and 268000 inhabitants. Its capital city is Funchal (Figure 10 left). A significant part of the island area is natural reserve park mostly covered by laurissilva forest, a subtropical laurel forest (Figure 10 right).

Madeiras' climate is considered Mediterranean, although it is common to encounter several microclimates on the island. On average the temperatures are between $17^{\circ} \mathrm{C}$ in the winter and $27^{\circ} \mathrm{C}$ in the summer, which means that heating and air conditioning are not commonly used for domestic purposes. This fact eliminates two of the main sources of electricity consumption in the household, a fact that flattens the difference in electricity consumption between winter and summer. Average sunlight is 10 hours in Winter, 11.5 hours in the Spring, 12 hours in Autumn and 14 hours in the Summer, providing a quite constant environment for electrical usage throughout the year.

There is a single electricity provider on the island, and the electricity consumed in Madeira is all produced on the island. The electricity comes from a mix of three thermal plants, four wind parks (located in the mountains), 10 hydroelectric stations (near the base of the mountains), and approximately 700 small and medium sized photovoltaic installations dispersed through the island. 

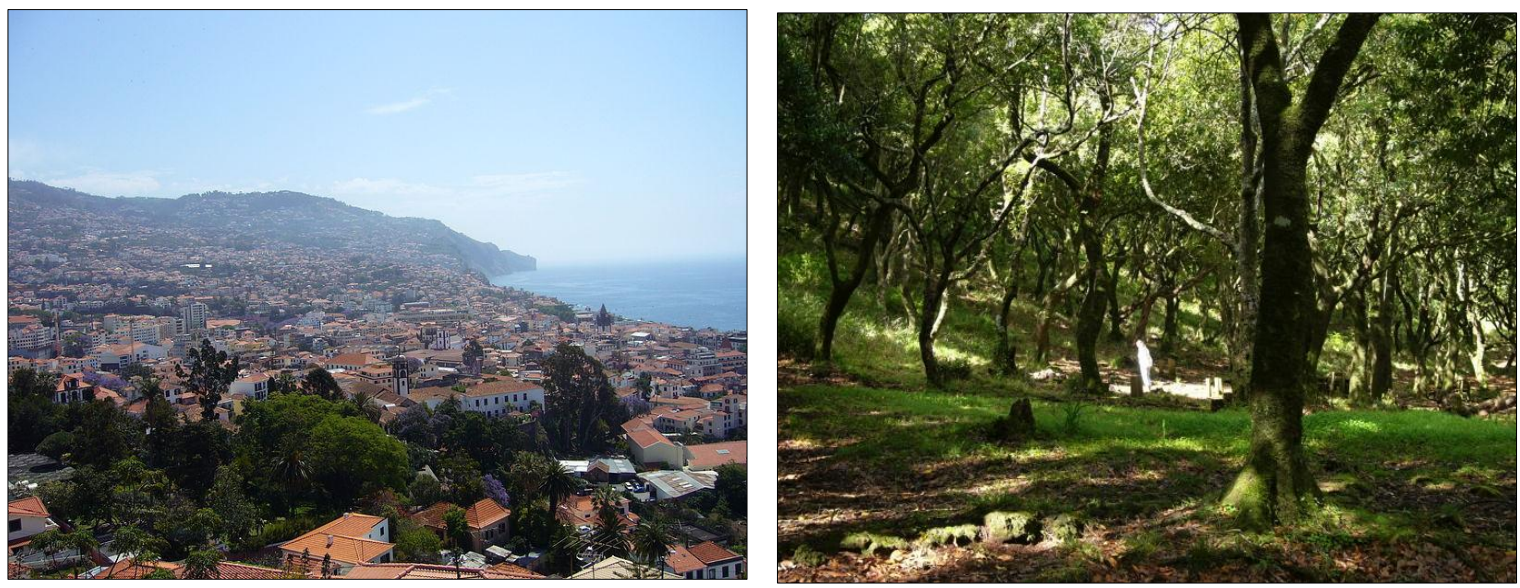

Figure 10: Left: City of Funchal, the capital of Madeira. Right: Madeira endemic laurissilva forest. 


\subsection{Research Hardware Platform}

In this section we describe the hardware used in the research experiments and deployments. Given the characteristics of the deployments in the wild the hardware research platform plays an important role, since the feedback itself is influenced by the underlying hardware that delivers it. There are two different hardware systems used in our experiments, which themselves represent two different eco-feedback modalities (fixed and portable). In the next sections we explain each platform in detail.

\subsubsection{Measuring electricity}

Measuring household electricity consumption is a central task to any project that aims at providing feedback on individual or collective consumption. There are currently several approaches to sense electricity that differ on cost, accuracy and scalability (see section 3.2). One of the most promising research approaches is NILM, which reads data from a single point and tries to monitor and disaggregate the consumption per appliance. The main assumption of most of the existing NILM approaches is that every change in the total load consumption of a household happens as a response to an appliance changing its state. Therefore, specific appliances can be isolated and their individual consumption calculated using complex signal processing and statistical learning techniques (Zeifman and Roth, 2011). Low cost and non-intrusiveness are the main advantages of the NIML approach thus it was chosen as the building block of the sensing infrastructure that supports this research (Pereira et al., 2012).

\subsubsection{Electricity consumption}

Electricity consumption is the electric energy used by appliances. It is represented in Watts (W) or Kilo-Watt $(\mathrm{kW})$ for real time usage. Aggregated energy consumption is often represented in Kilo Watt hour $(\mathrm{kWh})$ and it is an average of the watts or kilowatts used in one-hour larger aggregated electricity consumption is commonly represented as Kilo Volt Ampere (kVA), this metric is also useful to measure consumption from alternating current circuits. It is also common for domestic consumers to refer to their electricity consumption as their cost (e.g. monthly bill).

\subsubsection{Power Events}

One advantage of using NLM in our framework is the ability to detect power events. A power event is an abnormal change in the consumption normally associated with a household equipment 
being turned on or off. A power event is characterized by different variables such as the jump in real (the actual power used by devices) and reactive power (energy stored and released by inductors and or capacitors) consumption, timestamp and the transient (the actual shape of the jump in consumption). Figure 11 shows how a power events "looks" in a time series with the different components of electricity.

The power events were used as a metric in our eco feedback systems, for example they were displayed in the SinaisFromFanal system (section 4.4), as an additional or removed animal from the landscape. Power events are based on the consumption of the appliances, and they can also be used to analyse individuals' routines as we did for example in the evaluation of the What-a-watt system (section 4.7.8.3).

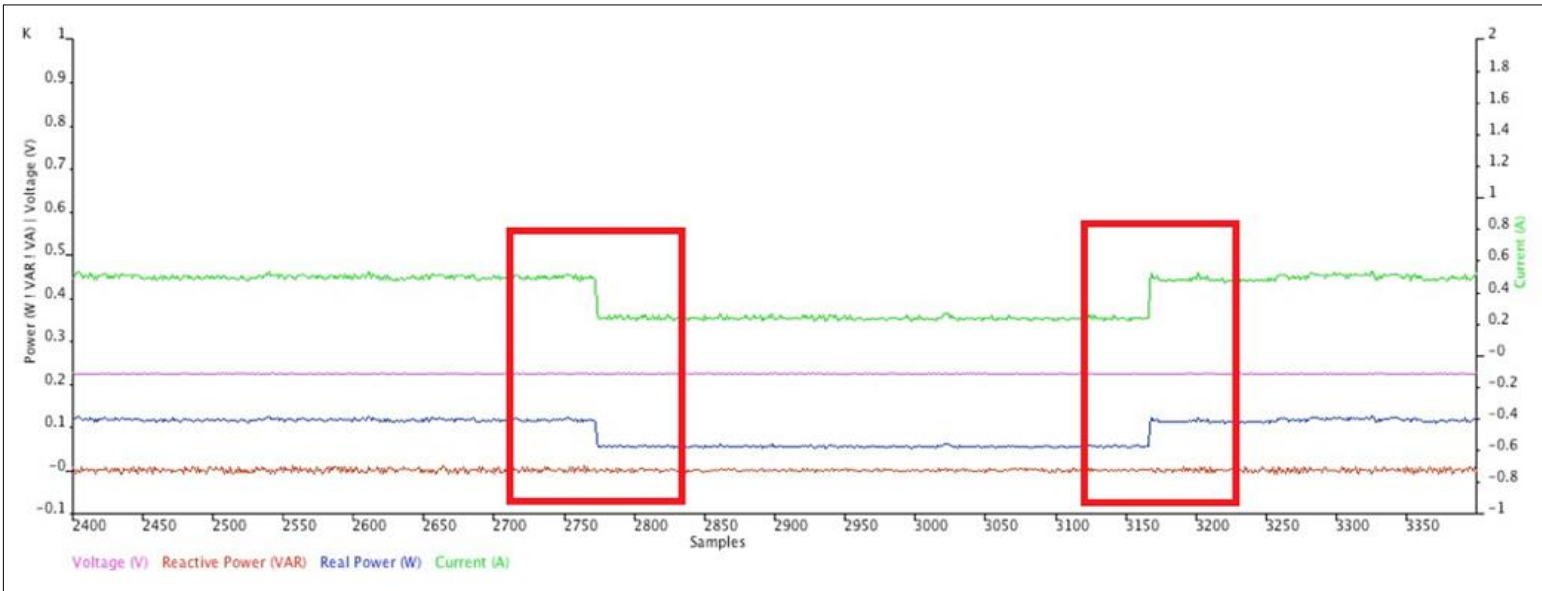

Figure 11: Time line of the different electricity components in a household, the power events are signed by red rectangles.

\subsubsection{Single house monitoring}

At the beginning of our research we explored how we could build a low-cost eco-feedback device that could be easily deployed. At a later stage we decided to adopt a custom made platform on which we could easily test new solutions (eco-feedback and algorithms) since commercially available devices were clearly not flexible enough. Commercial meters did not allow us to remotely update/monitor the installed software or to push the consumption data to a server, nor did it allow us the level of high frequency sampling needed for the more complex machine-learning algorithms.

After several attempts with custom hardware we decided to use a commercial netBook (Toshiba NB 300) as the base hardware for the household deployments. The netBook provided a cost effective platform for measuring energy consumption (through the sound board acting an 
analogue to digital converter), displaying visualizations (using the screen for in house visualizations), and detecting human action/presence (through the use of the keyboard, mouse pad, and webcam for movement and face detection). The whole hardware package (sensors + netBook) cost between 200 and 300 euros, which provided a very cost-effective and flexible solution for inhouse deployments, especially when compared to assembly of different components. The current was measured using a CT Clamp and the voltage using a $230 \mathrm{~V}$ to $0.5 \mathrm{~V}$ transformer (see Figure 12), both voltage and current signals were connected to the soundcard of the netBook which acted as the analogue to digital converter for the frequencies used in the electrical distribution in Portugal (50Hz).

The netBook had to be placed at the main power feed of the household covering the entire house consumption. This decision is a requirement for the implementation of the NILM algorithm, which aims at a breakdown of energy consumption into individual appliances while still maintaining the low cost while measuring the consumption at a single point. One disadvantage of this solution was the positioning of the netBook at the main feed which limits the visibility of the meter, since in older houses in Portugal the main feed is sometimes positioned in the basement or in hidden closets. Therefore, we targeted our deployments at households which had the main feed (fuse box) in a central position, normally behind the main entrance of the house or in the kitchen.

This single house monitoring device was the base hardware platform for the studies which used the SINAIS Power Meter 1.0, 2.0 and SinaisFromFanal eco-feedback systems (sections 5.3.2, 5.3.3 and 4.4).
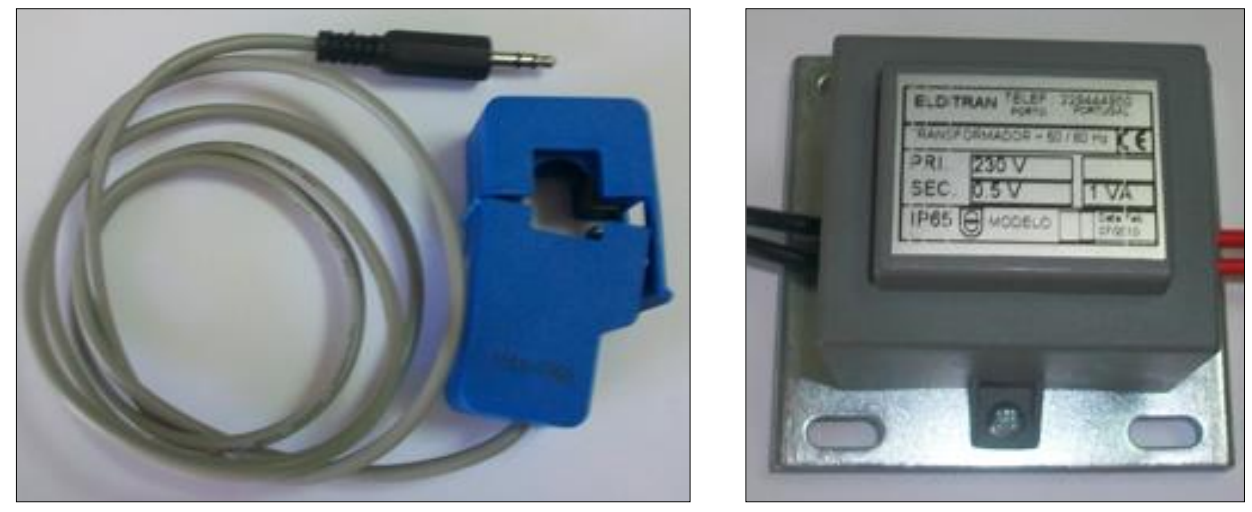

Figure 12: Current clamp and transformer used to measure current and voltage. 


\subsubsection{Multi house monitoring}

After the three first deployments and respective refinements of eco-feedback system (SINAIS Power Meter 1.0 and 2.0 and SinaisFromFanal, sections 4.3.2, 4.3.3 and 4.4) we decided to improve sensing hardware platform. The main reasons for this change are the following.

Firstly, the positioning compromises of the first version of the system had to be addressed as this type of stationary feedback is clearly limited since it is not accessible to all the household members at the time of decision-making (e.g. turning off a high-consumption appliance).

Secondly, the fact the system was connected to the main fuse box made some families worry about the safety of the device, and sometimes they would not allow children to use it.

Additionally several other requirements emerged from research efforts:

- The feedback should be accessible to all family members since family dynamics and communication will have an impact on the decision (Barreto et al., 2011). The system should allow for the comparison between different periods (hour, day, weeks and months) so that people can explore and better understand their consumption patterns.

- The system should also provide simple tips about energy conservation and best practices in promoting sustainable behavioural change.

- The hardware itself should also be aesthetically pleasing, as aesthetics plays an increasingly important role in interaction design, in particular when designing for homes and everyday lives rather than for the workplace (Petersen et al., 2004), it single handed can foster interest and promotes learning (Cosley et al., 2008).

\subsubsection{Sensing}

For the second version of our hardware we targeted apartment buildings, which by default have all the mains passing through the same location in the building (normally on the ground floor). This allowed us to sense the electricity consumption outside of the houses, addressing in this way the security concerns that were raised in the first two deployments. The netBook was also used in the second version of the hardware, it functioned as a server that worked together with a Data 
Acquisition Board ${ }^{1}$ (DAQ) placed at the entrance of apartment buildings, measuring the energy consumption of multiple houses from one single location.

The netBook runs a RESTfull web service that provides historic consumption data (e.g. daily, weekly, monthly consumption), as well as other metrics related to electricity consumption such as averages, or peak consumption periods. The real time consumption was broadcasted through a TCP socket using a custom-made communication protocol. Figure 13 shows the last implementation of our multi-apartment NILM sensing system. The image on the left shows the three voltage sensors (one sensor for each voltage phase) and the DAQ board, and on the right it is possible to see current clamps installed on the current conductors of the apartments monitored. Figure 14 displays the architecture of the infrastructure used to sense and distribute the consumption data.
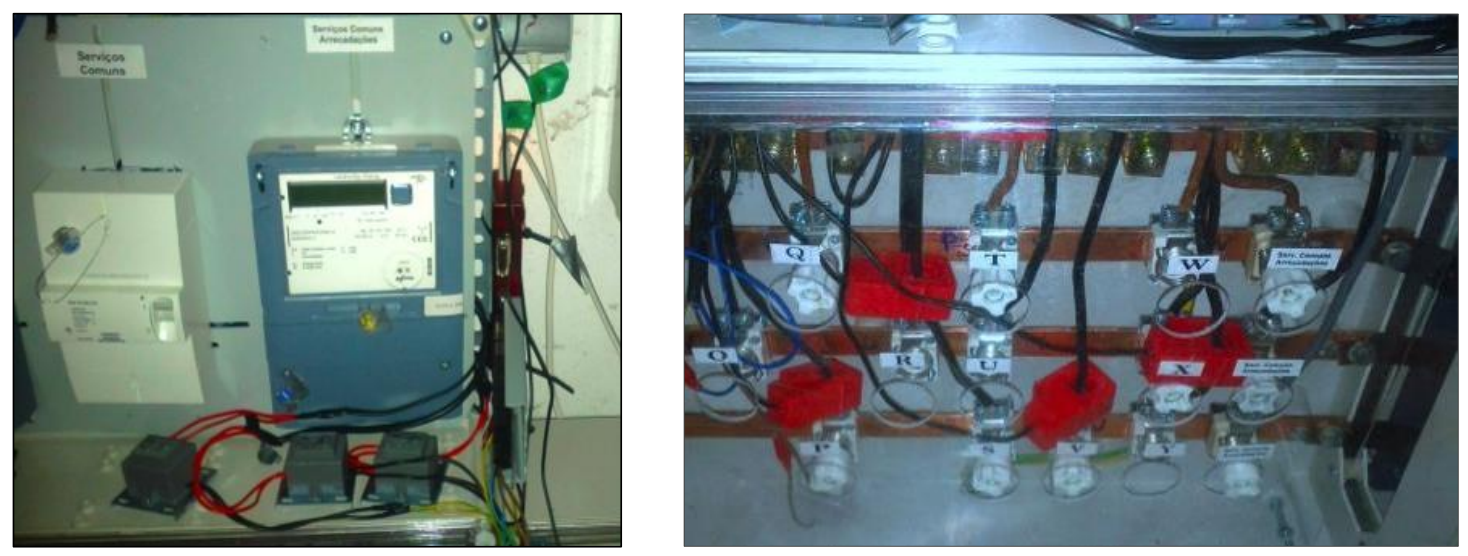

Figure 13: The system as it was deployed in one of the buildings of the WattsBurning study. On the left is the $D A Q$ Board and the voltage transformers. On the right is the current clamp sensing each house.

${ }^{1} \mathrm{http}: / /$ labjack.com/u6 


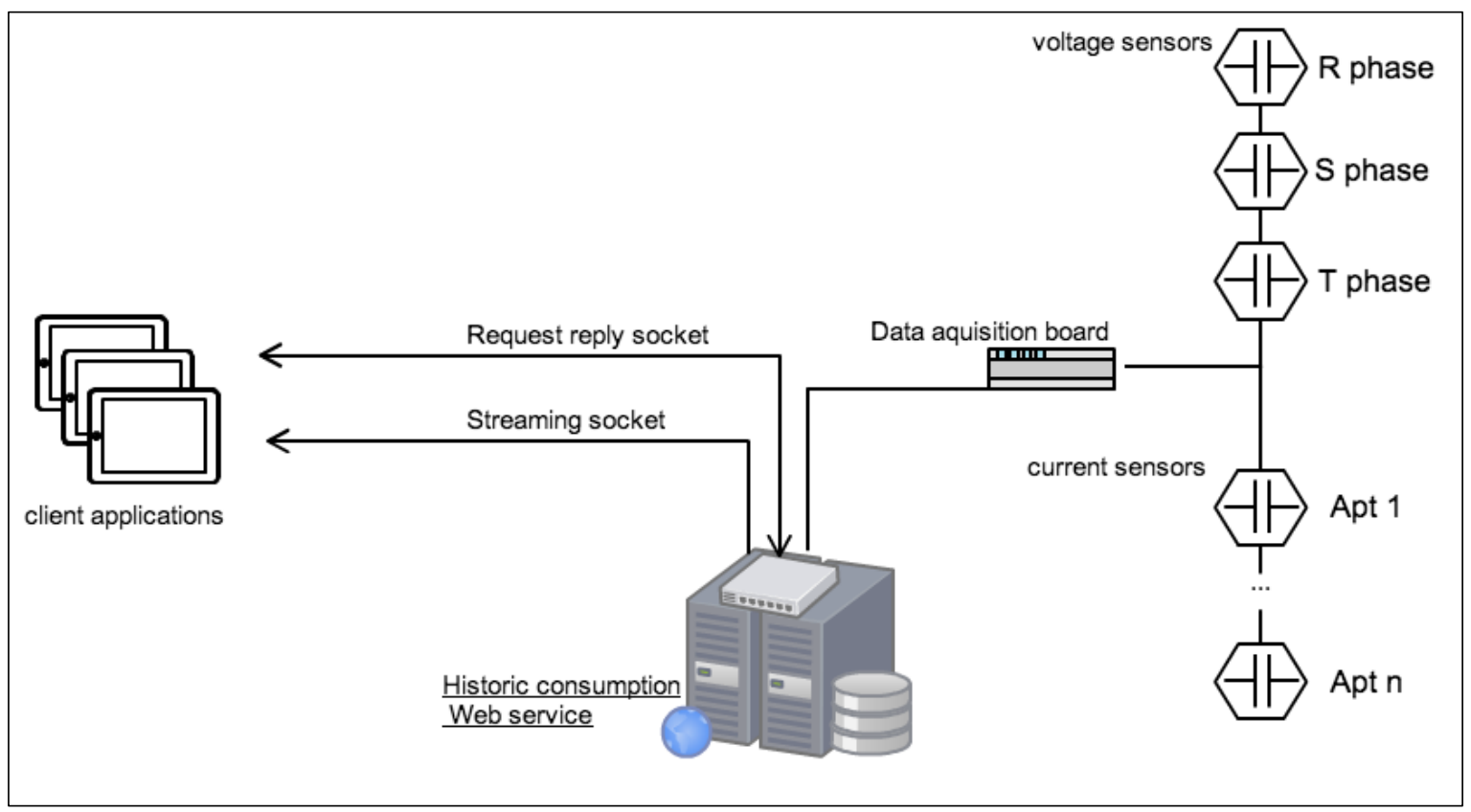

Figure 14: Overall architecture of the hardware + software platform.

\subsubsection{Feedback hardware}

Following the requirements described previously (section 4.2.5) the eco-feedback component was also updated. The eco-feedback hardware was updated to a 7', android tablet (see Figure 15). The tablet specifications allowed us to implement rich visualizations, which could be easily accessed from different areas in the house, or even outside. At the time of the deployment these devices (tablets) were still considered a novelty, and this helped our application fit in with the household as well as encouraging interaction. The tablet receives real-time consumption and historical data from the sensing framework. The real-time data is received using sockets and a custom-made communication protocol. Historical data is gathered by using the web-services described in the previous sections. The historical data is also stored on the tablet so the users can check past consumption without an Internet connection. The transitions between the different modes of operation are stored locally enabling the analysis of the usage patterns by the research team. 


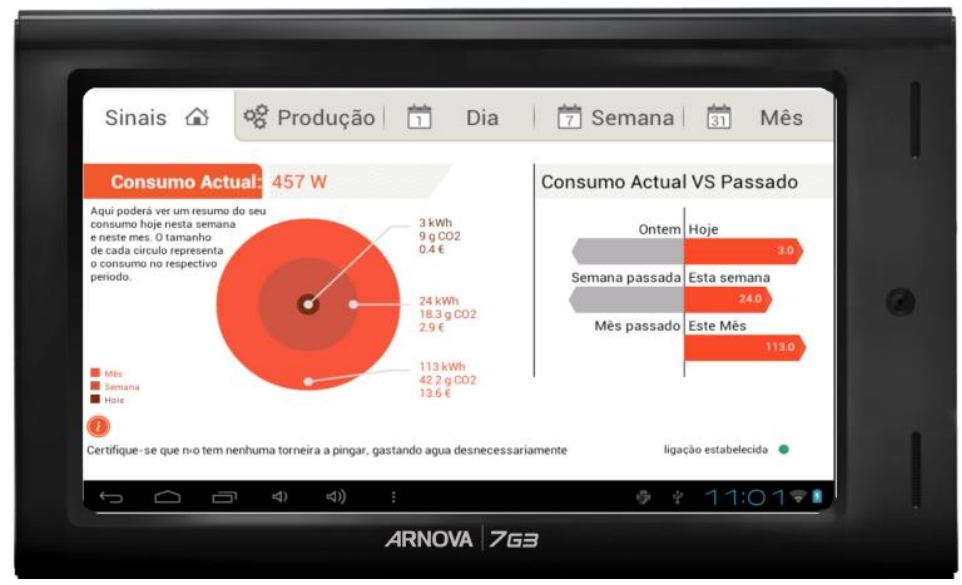

Figure 15: Android tablet used as a feedback client in the second version of our hardware. This particular tablet is running the What-a-Watt eco-feedback system. 
Research Studies 


\subsection{Background Work}

In the next section we present the design and implementation of the two first meters and summarize the research results. The findings reported in this section shaped the development of the following research in two ways. Firstly, the two initial exploratory studies exposed the limitations of commercial eco-feedback devices both as a research platform and as a product that would fit in the household. Secondly these studies provided initial evidence of the relapse effect of interaction with the eco-feedback systems, which we addressed in the remaining of this document.

\subsubsection{Conceptual design of the eco-feedback}

The first eco-feedback prototype was designed based on an evaluation of commercially available systems. The research team working on the SINAIS project deployed a set of commercial meters (Efergy ${ }^{2}$, CurrentCost $^{3}$ and Owl ${ }^{4}$, see Figure 16) and tested them with families for a two-week period (two weeks per family). Afterwards, families were interviewed using think-aloud methods in order to understand their relationship with the meters. This evaluation disclosed that users appreciated numeric information as well as comparisons charts. Additionally, we observed that participants did not feel confident enough to explore the different options of the most complex electricity meters fearing that they could break or unset the system configurations. Taking into account those concerns, in our next iteration of the system, we implemented an interaction feedback mechanism in which users could get information about the consumption without needing to interact directly with the device.

\footnotetext{
${ }^{2}$ http://efergy.com/eu/elitev1-monitor-163

${ }^{3} \mathrm{http}: / / \mathrm{www}$.currentcost.com/product-envi.html

${ }^{4} \mathrm{http}: / /$ www.theowl.com/index.php?cID $=185$
} 

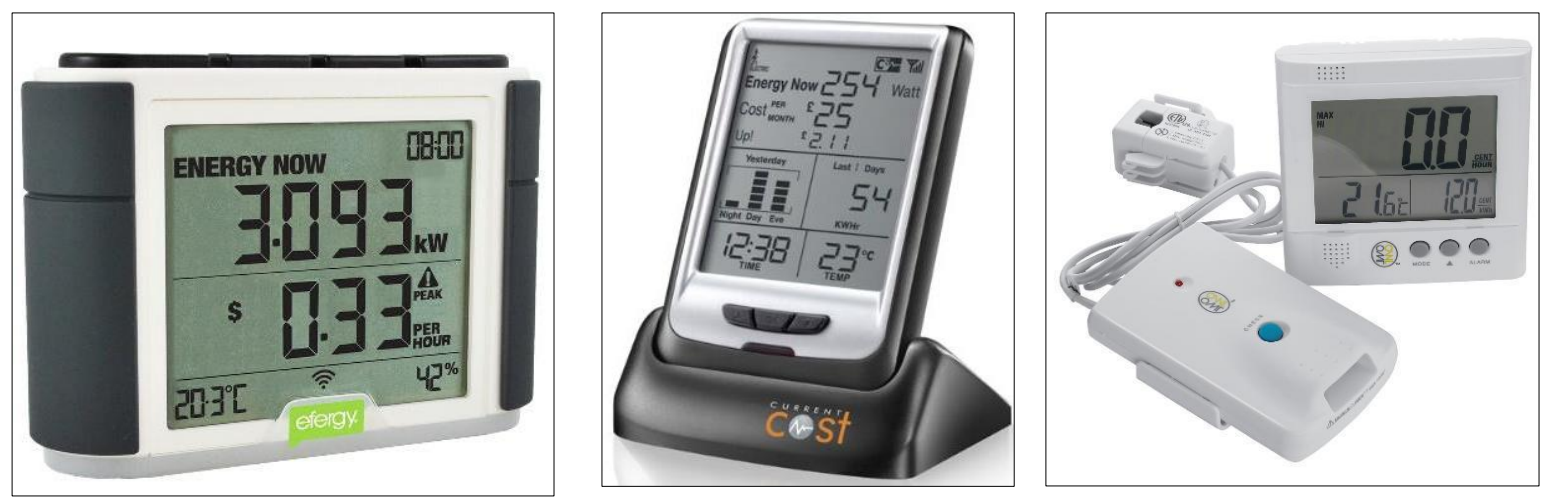

Figure 16: Electricity meter used in the pilot. Left: Efergy system; Middle: Current Cost eco-feedback system; Right: Owl electricity monitoring system.

The first eco-feedback prototype, was implemented using the Quartz Composer ${ }^{5}$ (QC) programming language. Quartz Composer is an Apple proprietary programming language which uses a visual programming paradigm, the prototyping nature of the language allowed us to rapidly implement several iterations of the system. This prototype ran on the single house-monitoring version of our hardware (see section 4.2.4) and at this stage the same Quartz Composer application was responsible for calculating the energy consumption and displaying the consumption feedback. The feedback was designed based on the assumption that the netBook would be positioned in a central place in the house and its display would remain open and visible (see Figure 17). This eco feedback system was organized into 3 modes motivated by our previous observations:

- Hidden mode, when there is no movement next to the laptop and it only records data.

- Attract mode displays a first level of information when it detects human activity or an electrical event (appliance turning on or off).

- Active mode offers a chance for direct user interaction with the system in order to provide additional information.

The hidden mode of the application did not display anything on the screen, although the application was still recording consumption data. In this mode the webcam is active, sensing movements in front of the meter. The attract mode of the application (Figure 18 left) shows a spectrum-like graphic with a representation of the last 8 hours of consumption and with this abstract visualization we aimed at providing feedback at the first glance of the user. The active mode (Figure

${ }^{5} \mathrm{https}: / /$ developer.apple.com/technologies/mac/graphics-and-animation.html 
18 right) shows the above-mentioned spectrum and a line chart with the consumption of the current day. Also in the active mode the user can opt to query the consumption further, and the application will present the user with 2 more charts representing the consumption in the current week and month (Figure 18 bottom).

The Quartz Composer prototype was never deployed within real households, but the system was tested in several lab evaluations, between students, professors and researchers. The design choices and findings that emerged during the development of this prototype were later used in other feedback visualizations.
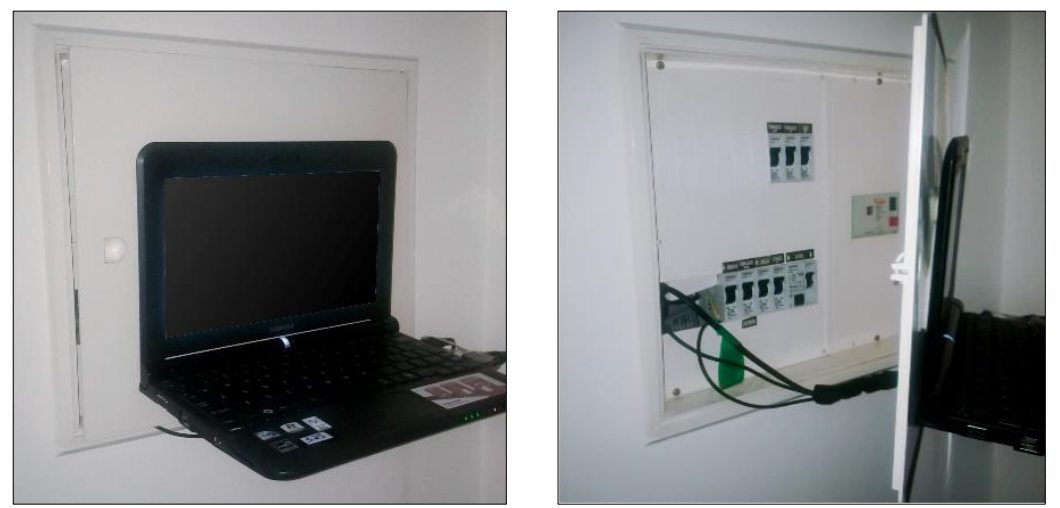

Figure 17: Notebook installed at the main fuse box of the household. This configuration was used in the first version of our hardware.
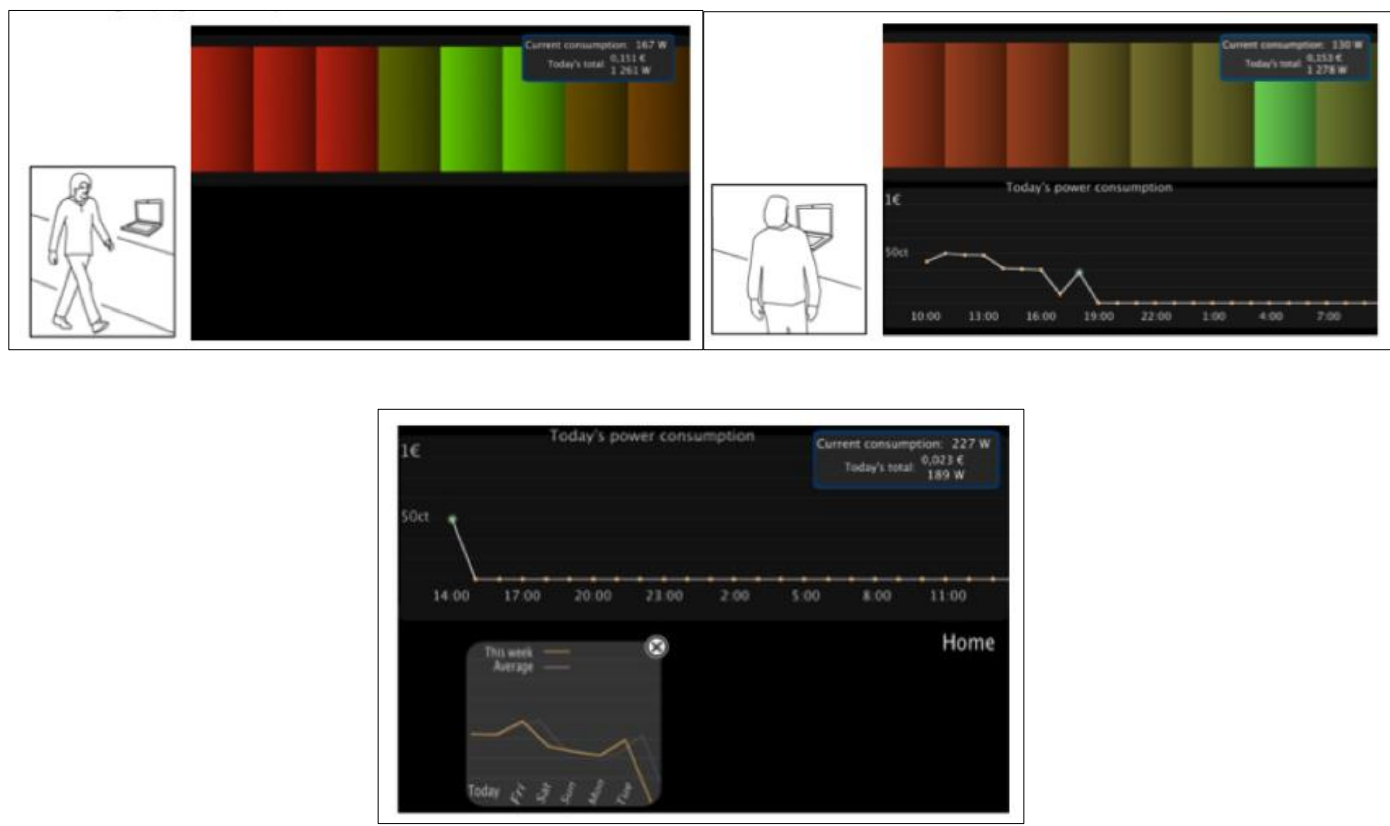

Figure 18: Top Left: Attract mode of the system, Top Right and Bottom: Different options presented in the Active Mode of the prototype. 


\subsubsection{SINAIS Power Meter 1.0}

After the first exploratory studies described in the previous section, we started to refine and build the system that could be deployed in households for a long-term study. The Quartz Composer approach had to be abandoned since it is proprietary single platform software and would hinder a wide deployment, and instead Adobe AIR 2.06 was used. The Adobe AIR 2.0 software while still being proprietary allowed for the system to be used on different platforms (MacOS, Linux), and easily ported for web if needed.

This version of the system also completely separated the front end module and the data acquisition (DAQ) module of the system. The first module provided feedback regarding several metrics related to electricity consumption, while the second takes readings from the sensors and calculates the energy consumption. The communication between these two modules is made through a socket, and a shared database. The meters were connected to a private WLAN assembled by our research team with the help of the local electricity company and Internet providers. This was possible since most of the households belonged to apartment buildings close to each other. However, several meters were installed further away and could not connect to the WLAN and for these cases they used the household Internet connection (where available) to post data to the server.

\subsubsection{Visualization}

The visualizations used in the front end were similar to the ones explored in the Quartz Composer prototype (see Figure 18). It presented real-time and historical consumption using bar charts. For this feedback we used three different modes of operation:

- Idle-black screen.

- Attention - spectrum-like graphic showing the consumption in the last 8 hours compared with an average for the house (Figure 19 top left).

- Detail - several charts showing and comparing the consumption during the day, week and month. In this mode the user could actually directly interact with the system to explore the energy consumption of different days (Figure 19 top right and Figure 19 bottom).

${ }^{6} \mathrm{http}: / /$ www.adobe.com/products/air.html 
In the Idle mode the front end of the system is "turned off". In this mode the netBook webcam is working as a movement sensor and it only changes the feedback state if someone passes or is near the system. The Attention mode is triggered when the system senses that someone is near the netBook. In this mode the feedback displays a set of eight bars that comprise a colored gradient representing the consumption of the last eight hours - the colours of the gradient range from green to red depending on past consumption (see Figure 19 top left). Furthermore, in the Attention mode the system displays the current consumption (in Watts) as well as the day's total (in $\mathrm{kWh}, \mathrm{g}$ of $\mathrm{CO}_{2}$ and Euros). In the Detail mode users are presented with the same panel that shows the real-time and day's total consumption, additionally the system will display the current day's consumption in a bar chart (Figure 19 bottom left), the user can also view the consumption of the current week (Figure 4 top right) and compare different days (Figure 19 bottom right). This mode is triggered when the netBook senses that someone is looking directly at the feedback (using a face detection algorithm ${ }^{7}$ ), or when someone clicks on the netBook's mouse pad.
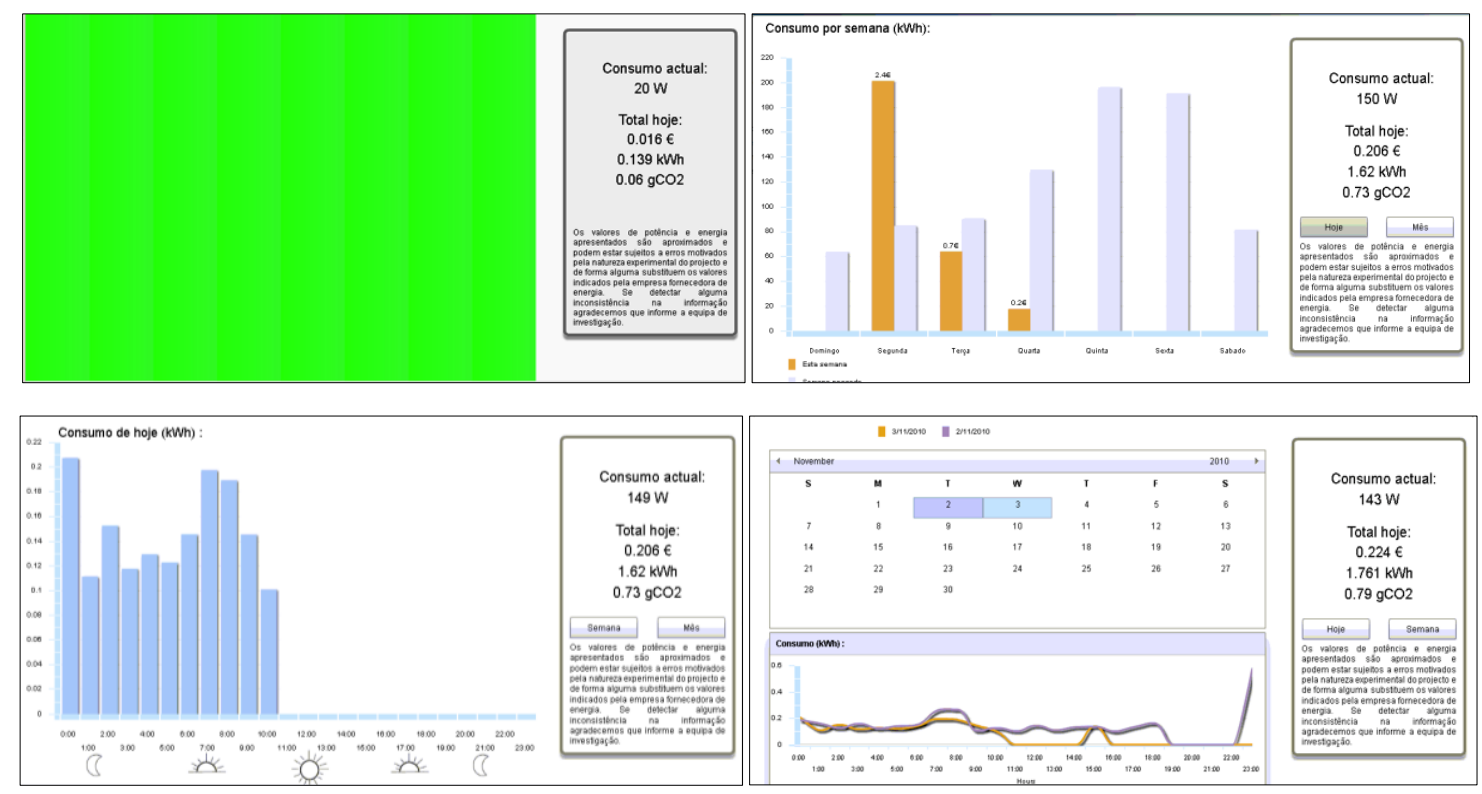

Figure 19: Different views of the SINAIS Power Meter 1.0: top left: spectrum graphic with the consumption of the last 8 hours; top right: consumption throughout the week and comparison with previous week; bottom left: daily consumption; bottom right comparison between different days of the month.

\footnotetext{
${ }^{7}$ Haar Feature-based Cascade Classifiers from Marilena framework (http://www.as3gamegears.com/computervision/marilena/)
} 


\subsubsection{Evaluation}

The system was evaluated part of the SINAIS research project through in-the-wild study in the city of Funchal.

\subsection{Sample}

The electrical distribution company of Madeira gave us access to data for all the 46500 domestic consumers registered in the city. We divided the consumers into four levels of annual consumption. Using the aggregated data, we selected an apartment building that fits the city average in terms of the average consumption profile. The apartment buildings were selected randomly from a high-density living area close to the University campus. We surveyed all the people in three buildings and recruited about 30 families that voluntarily agreed to participate in the study. During the deployment, a qualified technician from the electrical company installed the netBooks and the sensors (see Figure 12). Each family filed a detailed survey upon installation and received a compensation of 10 euros per month to participate in the study, which also involves several qualitative interviews to assess the progress, for this first study 21 houses where selected and all the consumption groups were represented. Families involved in this deployment were referred to as F1 to F30. Appendix 9.1 and 9.2 present the two questionnaires that were filled by users before the study.

\subsubsection{Collected data}

The deployment of the SINAIS Power Meter 1.0 used the first version of our hardware (section 4.2.4 presents de deployed infrastructure). The sensing platforms samples data and calculate averages for current, voltage, active and reactive power every 30 seconds. These measurements are used to calculate average (e.g. daily, weekly or monthly) values for feedback to the users and also stored in the local database and data warehouse for analysis.

\subsection{Quantitative evaluation}

The system was deployed in 21 houses for a period of nine weeks in the city of Funchal (more on the location in section 4.1). This was our first experience deploying and implementing a system such as this one, and the study was highly exploratory. The quantitative data gathered in this study showed that after the nine weeks there was a $10 \%(\mathrm{M}=10 \%, \mathrm{SD}=6.13)$ aggregated decrease in 
consumption (considering all houses). However, the decrease was not consistent over time, this way, in the rest of this section we analyse in detail the qualitative data of this first deployment.

As we mentioned previously our system also recorded information from user-generated events. These include movement and user presence detected by the webcam of the netBook (motion and face events), mouse clicks and transitions in the interface (mouse events). We considered motion events detected by the webcam of the netBook to be non-intentional interactions, since the user could just be passing near the system. We considered intentional events direct interactions with the system such as movements in the touchpad, clicks in interface elements or when the user actually stopped in from of the netBook and looked at the screen.

After the fourth week there is a decline $-42.8 \%$ over week 1, (see Figure 20) in non-intentional user events and this decline is steady as we progress with the exception of week eight, which corresponds to a peak in movement events. The weekly average user generated events (of motion, face and mouse) was $2409.1(\mathrm{M}=2409.1 \mathrm{SD}=584.4)$. The high standard deviation is explained by considerable differences among houses, seven houses had less than 100 user events over the period of nine weeks, while eight houses had between 100 and 500 user events, and finally six houses had more than 500 users' events over the same period of time.

However, the majority $(80 \%)$ of the recorded events were motion events, which we have considered to be "non-intentional". This way we focus on mouse and face events (intentional events) and found an average of $33.9(\mathrm{M}=33.9, \mathrm{SD}=44.4)$ events per house over the nine-week period. Creating groups by percentiles we clustered the households as follows:

- Group A with an average of less than 60 non intentional user events per week.

- Group B between 60 and 165 events non intentional user events per week.

- Group C above 170 events non intentional user events per week.

We then analysed these three groups in more detail, group A for houses with low user events, group $\mathrm{B}$ moderate and group $\mathrm{C}$ for high number of user events. Group $\mathrm{C}$ generates a substantial number of events when compared with groups A and B. More importantly the number generated events remain high over the nine-week period still with a significant drop after fourth week. The other two groups tend to decrease slowly over time and reach marginal values.

Houses in Group A have a very low number of user events, an average of 19.7 ( $M=19.7$, $\mathrm{SD}=10.0$ ) intentional events. The overall energy decrease over the nine week period is of $23.7 \%$. 
Houses in Group B have a moderate number of user events, an average of 86.4 ( $\mathrm{M}=86.4$, $\mathrm{SD}=25,0)$ mouse and face recognition events. The overall energy decrease over the nine week period is only $3.4 \%$.

Finally houses in Group C have a very high number of user events, an average of 388.8 $(\mathrm{M}=388.8, \mathrm{SD}=193.0)$ intentional events. The overall energy decrease over the nine-week period is $6.4 \%$ in this group. This group is also the most stable of them all in terms of events, which is not surprising given the higher level of events.

One potential explanation for the behaviour between the different groups would be that each group also corresponds to substantial different energy consumption. However, groups $\mathrm{B}$ and $\mathrm{C}$ have almost the same average weekly consumption per house $B=20.3 \mathrm{kWh}(\mathrm{M}=20.7 \mathrm{kWh}, \mathrm{SD}=2.2 \mathrm{kWh})$ and $\mathrm{C}=23.2 \mathrm{kWh}(\mathrm{M}=23.2 \mathrm{kWh}, \mathrm{SD}=1.2 \mathrm{kWh})$, while group $\mathrm{A}$ is substantially lower with average $11.7 \mathrm{kWh}(\mathrm{M}=11.7 \mathrm{kWh}, \mathrm{SD}=1.0 \mathrm{kWh})$.

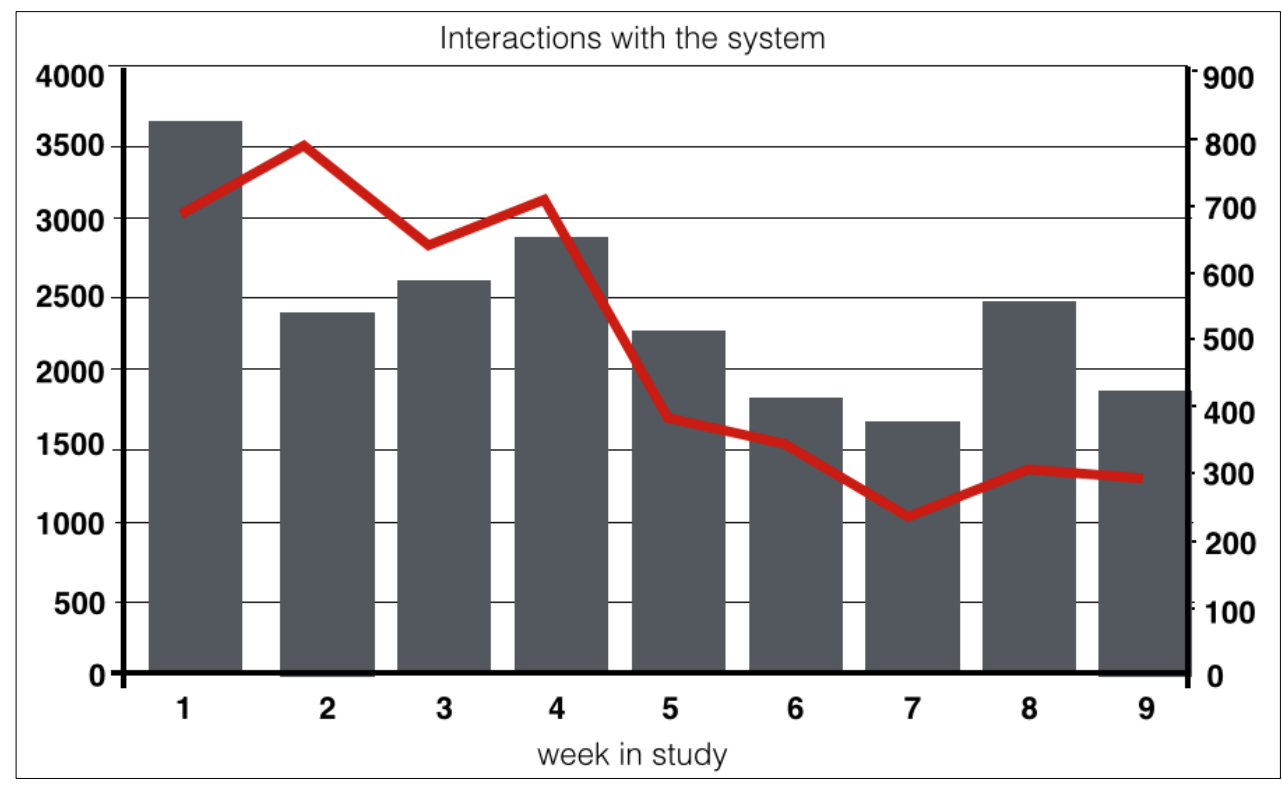

Figure 20: Interactions with the SINAIS Power Meter 1.0 system. The grey bars represent the sum of all interaction and the red line represents the intentional interactions.

During our interviews we learned that this decrease in attention was partially justified by the fact that participants were becoming more knowledgeable about their consumption, as the wife from F28 pointed out:

"We would check our consumption more often initially until we got a rough idea or perception of what our consumption was but after that it would less frequent" (Wife, F28) 


\subsubsection{Conclusions}

This first study presented the preliminary results of an eco-feedback study with 21 houses during a period of nine weeks. Overall the houses in the pilot saw an overall reduction of approximately $9 \%$ of energy consumption. Although we consider that consistent results need a wider timespan we could already find interesting patterns in user events. We also observed that house that interacted more with the eco-feedback accomplished a bigger saving in energy consumption when comparing with houses that used the system to a lesser extent.

Our initial research results confirmed the short-term effectiveness of eco-feedback technology. We observed that houses with the eco-feedback system installed reduced their consumption as reported by others (Colley et al., 2013; Fischer, 2008; Froehlich et al., 2010). A second results confirmed that users with more intentional interactions with the eco-feedback system were also the ones that presented more energy savings.

Further steps in our research explored the above-mentioned observations in more details, by testing the effectiveness of eco-feedback in the long term.

\subsubsection{SINAIS Power Meter 2.0}

Through the previous deployment we gathered a lot of valuable insights as to how consumers adopt eco-feedback systems, and more generally about how to deploy such systems in the real-world scenario (see section 4.3.2.2).

The second version of the system was also running on the single house-monitoring version of our hardware (see section 4.2.4). The installations were remotely updated to the new version of the feedback, and users were notified by phone when the system had been updated.

\subsubsection{Visualization}

The new eco-feedback interface was developed with the help of a designer following a set of "guidelines" extracted from the research literature. For example, Darby argues that the feedback should be displayed accordingly to its importance: the current energy consumption should be the central focus, historical information, averages, energy peaks and $\mathrm{CO}_{2}$ are secondary, (Darby and et al., 2006). Therefore, the visualization showed the household consumption by hour, day, week, month and year, however the current consumption is placed at the centre of the visualization. 
We used a gauge analogy to display the feedback (Figure 21), the gauge was divided into time slots depending on the time period i.e. 24 slots representing the 24 hours of the day, 31 slots representing the 31 days of a month, and 12 slots (one for every five minutes) for the hourly representation. Every slot of the gauge was color-coded according to how the consumption in that time period compared with the household baseline (for example in Figure 21 bottom left, the hour between 9:00 and 10:00 had higher consumption than any other hour on that particular day). In the centre of the gauge the real-time consumption was displayed. Each tab of the interface had three boxes that showed total consumption (in $€, \mathrm{kWh}$ and $\mathrm{gCO}_{2}$ ) in the correspondent time period, a comparison with the previous homologous period and a simple tip regarding sustainability (tips retrieved from different sources online, for example the Stepgreen social network (Mankoff et al., 2010). For this version of the system the face detection interaction feature was abandoned, since it was highly CPU intensive compromising other concurrent tasks. However, the motion detection feature was kept when no motion was detected next to the meter all the information boxes would disappear and the system only showed the gauge with real-time and hourly consumption (Figure 21 bottom Left).
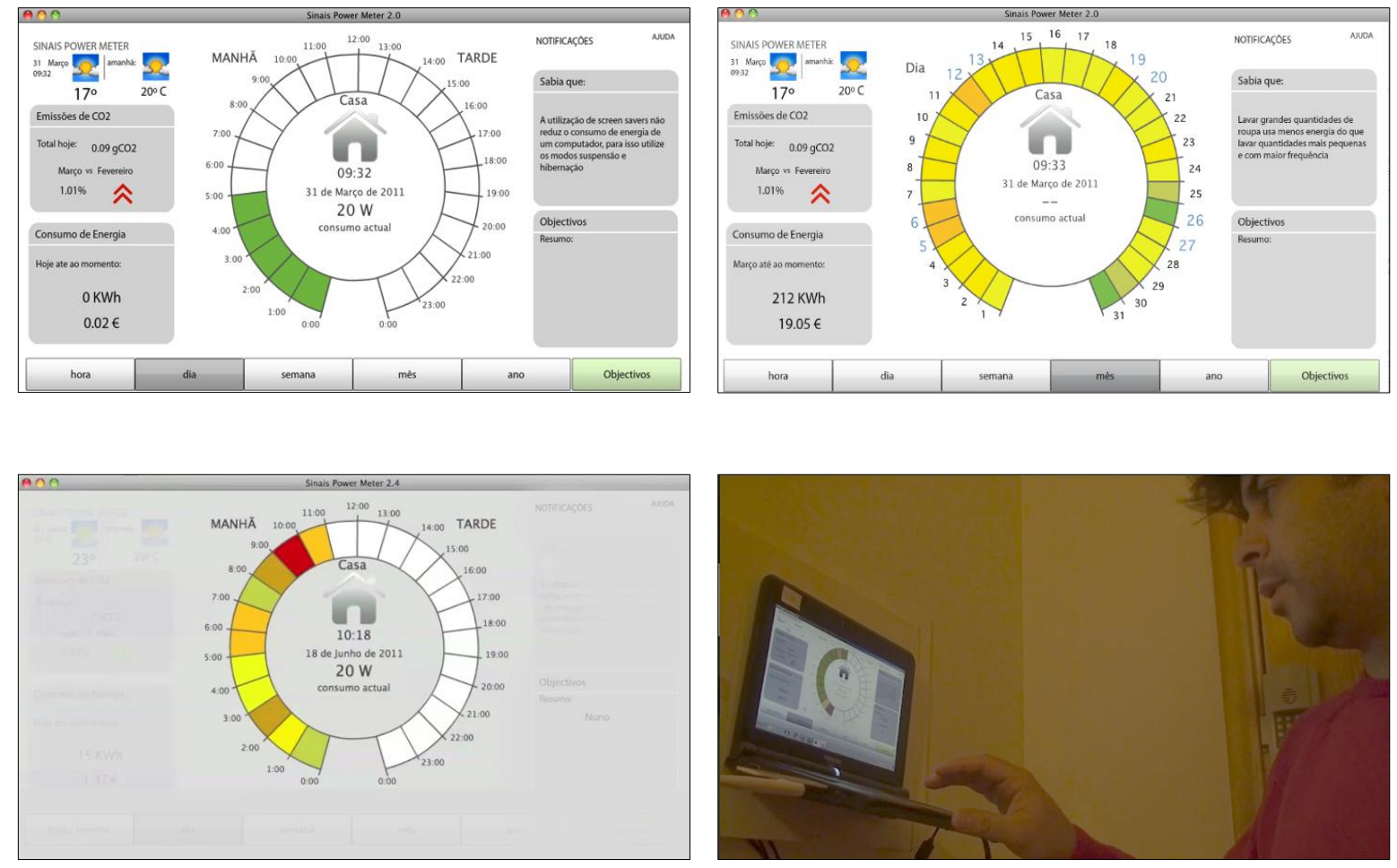

Figure 21: Second version of the eco-feedback system. Top right: Daily consumption; Top left: Monthly consumption; Bottom left: View of the system when no movement is detected near the meter after 2 minutes; 


\subsubsection{Method}

The second version of the SINAIS Power Meter was deployed in a sub sample of the individuals selected to test the first version, the update was done remotely and families were informed by phone that the system had been updated. Thirteen houses were selected and they used the system for 52 consecutive weeks. Consumption and interaction with the system data was collected (see section 4.3.3.2 for more about the collected data). Families were formally interviewed twice during this period, one month after the start of the study and in the middle of the 52 weeks. In the following sections we present our quantitative and qualitative evaluation for the period of the study.

\subsubsection{Quantitative evaluation}

\subsection{Consumption through the study}

To verify if there was any decrease in electricity consumption we ran a correlation between the energy consumption and the week of the study, $r(52)=.026, p<.0001$. This means that the consumption was correlating positively with the week of the study but was quite weak $(r=0.026)$. One possible cause for this was that the subjects had already been in contact with the previous system (section 4.3.2) and made small adjustments in their routines to reduce their overall consumption. To test this result further we ran a linear regression algorithm and got the following equation relating the consumption and the week of the study:

EnergyConsumption $=0.687 \times$ WeekInStudy +423.2

By simply assigning the value 0 and 58 to the WeekInStudy variable, we get a difference of about $40 \mathrm{Wh}$ between the first week and the last week, which is small (a small 50W lamp can spend that amount of energy in about an hour). As result we can safely assume that there was no real change in the consumption during the course of the study. To explore those results, we individually asked families about any change in the consumption, most of them confirmed that there was no real saving in the electricity bill at the end of the month, but some of the families noticed a decrease.

\subsection{Interaction with the system}

Since we used the same households for the first and second deployment the subjects were already familiar with eco-feedback devices. They used the previous version (the SINAIS Power 
Meter 1.0 system) for three months, and there was a noticeable drop in interaction with it after four weeks. To verify if this phenomenon would be present in the second deployment we assumed that the number of interactions would negatively correlate with the week of study. The Pearson's correlation for this data returned a significant negative correlation, $(\mathrm{r}(52)=-.163, p<.0001)$. We also ran a linear regression algorithm and got the following equation:

NumberOfInteractions $=-0.014 \times$ WeekInStudy +0.871

Also regarding the interaction with the eco-feedback system, it was shown that the greatest decrease in interactions with the system occurred in the first four weeks like it was previously reported (section 4.3.2.4). For this the dataset was separated in two groups (the first four, and the remaining weeks) and we ran two regressions analyses. The results showed that the slope in the first four weeks $(-0.054)$ is greater that in the rest of the study $(-0.013)$. These findings indicate that the decrease in that period is greater that in the rest of the study.

When interviewing the users, we enquired them about this decrease of interest in the system. Families referred that lack of time in their routines meant that the system was gradually being relegated to a second plan, others feel like after a few weeks they already had a good perception of their consumption:

"We didn't ignore the device I would look at it everyday. What I noticed is that we achieve an average of consumption (...) because we use around the same devices all the time and our attention to the device might decrease, we don't analyse it so carefully. " (Father, F18)

“I haven't paid much attention to it lately because I haven't had time that's the problem. I was the one using more but the last 9 months, we just had a baby and it has been a lot of work" (Father, F16)

\subsection{Number of People vs Energy consumption vs Power events}

We conducted a different analysis to understand the difference in consumption between the households. In particular we explored the relationship between the number of household members, the energy consumption and the number of power events (see section 4.2.3 where we describe what is a power event in the scope of our work). Like in the previous analysis these relationships were investigated using correlations and linear regressions.

The results of the analysis were two significant correlations between energy consumption against number of household members $(r(24)=.273, p<.01)$, and number of power events against 
number of household members $(r(24)=.119, p<.01)$. The results showed that both energy consumption and the number of power events correlate with the number of people in the house. To better understand the results from the previous correlation we ran two Linear Regression analyses, to predict the energy consumption and number of power events, based on the number of household members. The obtained equation are presented below:

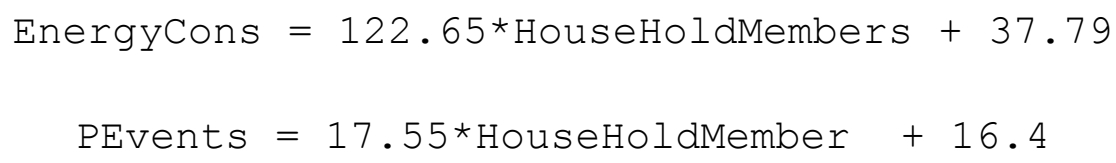

Looking at the slope value of the two equations it is clear that households with more occupants have higher consumption. Yet, since the number of the events also increased with the number of members. We can deduce that the increase in the consumption is caused by a higher number of appliances used in the house, or because they are used more often.

To explore these relationships even further we subsequently, summed the power events by hour of the day and calculated the average number of events per person in each household which returned a normalized number of power events per household member ( 24 points). The normalized power events were then correlated with the total number of events in each house.

If the number of household members highly influences the total number of events then the difference in variation between the amount normalized events and the total events number would be high, subsequently the correlation would be weak. On the other hand if the number of household members did not influence the number of events the difference in variation between the values of the normalized power events and total power events would be small this correlation would be strong.

This analysis returned a strong positive correlation $(r(24)=.892, p<.01)$. Based on the result, we can speculate that the appliances responsible for most of the power events (and energy consumption since they correlate) are present in every house.

\subsubsection{Qualitative evaluation}

The qualitative evaluation of the SINAIS Power Meter 2.0 was based on two rounds of semistructured interviews with all the families involved and several informal interactions with the. The discussion was centred in how the system was accepted in the household and if it allowed any savings in the electricity bill. Interviews were transcribed to text and families' answers were arranged in related themes, our thematic analysis uncovered the following topics: Interactions with the system, Changes in Consumption and Power consumption vs Power Events. Next in the sub chapter we 
present each topic. The number that was assigned to them at the initial sample selection identifies families, and it ranges from F1 to F30.

\subsection{Interactions with the system}

Our interviews with families further revealed what the quantitative data showed us, there was a peak in the interest of users with the system when the SINAIS Power Meter was updated to the 2.0 version. Consumers revealed that after they got familiar with the system, they got a rough idea of their consumption. Which meant that they did not have to check the system constantly, like F28 and F19 pointed out:

“...more often initially until we got a rough idea or perception of what our consumption was but after that it would less frequent..." (Wife, F28)

"...If people have the same routines and the same consumption, I think people do not feel they need to check the device constantly..." (Husband, F19)

However, some families really engaged with the system and used it every day in their routines, however even these families admit that after a while the frequency of the interaction decreased, like family F19 explained.

“...We didn't ignore the device I would look at it every day. What I noticed is that we achieve an average of consumption, we think we have the usage, because we use around the same devices all the time and our attention to the device might decrease, we don't analyse it so carefully..." (Husband, F19)

\subsection{Changes in Consumption}

During our interviews it was important to assess if individuals were aware of any change in their electricity consumption. Not all consumers felt that the system promoted savings in the household, since they already were careful with over consumption, like the mother from F27 pointed out:

“...We didn't notice major changes. We already did a couple of things we would already disconnect some devices, toaster or radios..." (Wife, F27) 
On the other hand, other families stated the SINAIS Power Meter 2.0 allowed them to closely follow their consumption, and eventually implement small adjustments to it. F28 took this action to the point where they changed all lamps in the household:

"...My routine was changed in the point where I changed all the lamps in the house to more energy efficient ones. I started to turn off the lights more often because I could see the impact of it so I had to do it..." (Wife, F28)

In general, our interactions with families revealed that the biggest long lasting effect of the Power Meter 2.0 is an increase in awareness of electricity consumption. Consumers became more aware of bigger household wide habits, but also aware of the consumption of particular devices, as it was mentioned by F4.

“...I think the changes were mostly on making us more aware of devices we used and habits we had. We had some bills that were a little expensive and we started to reduce some consumption (...) Now I feel we have reached a constant value, we pay around the same amount each month..." (Wife, F4)

\subsection{Power Consumption vs Power Events}

Although the SINAIS Power Meter 2.0 provided information regarding aggregated energy consumption (wattage consumption), families often discussed their consumption by referring to their usage of appliances. For example, mother from family F27 points that she need to be more careful in how they use the dryer and washing machine.

"... When we are at home we spend more. It's not easy to reduce. I try to be careful with the machines, how I use them..." (Wife, F27)

Even though the SINAIS Power Meter 2.0 did not present disaggregated energy information, consumers were able to infer individual appliances consumption by looking at the total load. The fact is that the only way for an individual to interact with electricity is by using electric appliances or equipment (e.g. light switches, microwaves). Consumers also confirmed this observation (F19 and F8).

"...It helped us to see some devices were consuming more than we initially thought and it changed the way or time we used those devices ..." (Husband, F19) 
"...We reduced the lights consumption and we unplug the television now. The system made a difference here..." (Wife, F8)

\subsubsection{Discussion}

Our results are in line with what we had found in the evaluation of the previous version of the system (SINAIS Power Meter 1.0), and with results from literature. There were in fact savings in consumption (which transacted from the first study), we found that these savings are the result of consumers adjusting their routines or replacing certain equipment (e.g. light bulbs). We argue that the increased awareness promoted by the Power Meter helped consumers made those adjustments, again this result is in line with literature reports.

During this study we also observed a decrease in usage of the SINAIS Power Meter 2.0 over time, this observation was confirmed by our quantitative analysis and by our personal communication with families. We argue that a possibility for this loss of interest is that after a while both the SINAIS Power Meter 2.0 interface and the families routines (that are related to usage of electricity) are rather "static", and the biggest triggers to go back check the system is if the family acquired a new appliance, made any significant change in their routines (for example the birth of a child) or if the Power Meter itself introduced new features.

Lastly in our formal and informal conversations it became clear the families' often used the usage of appliances vocabulary regarding electricity consumption, it was the way individuals interact with the electric grid and it made sense that families talked like that. Furthermore, your statistical analysis confirmed this strong relationship, which we believe, might provide an opportunity for feedback.

\subsubsection{Conclusions}

The scope of this study was limited to aggregate data and important results can surface when we compare different houses or different conditions within the same house. We found that events are related with energy consumption and this preliminary research might indicate that the increase in consumption is normally associated with more appliances. We did not found any significant difference between consumption on different days of the week but there was a difference in the power events, which might indicate different usage patterns during the week. We also confirmed what other researchers found, users lost interest in the system after a while and even the small updates delivered over time were not enough to prevent this. From informal conversations with users it was 
noticeable that our system helped increase their perceptions on energy consumption that was perhaps the biggest contribution of our work with the SINAIS Power Meter 2.0. To better understand our speculations, it would be important to use more qualitative data like investigate user's routines during the period of the study. We also plan on extending the level of energy of desegregation of our system to measure the added value of this feature in terms of energy savings. 
Research Studies 


\subsection{Shift in the feedback paradigm, mapping the consumption to the local landscape: SinaisFromFanal}

This chapter describes efforts moving away from traditional eco-feedback into representations that connect users with long-term consequences of energy consumption. We named this ecofeedback SinaisFromFanal connecting the name of the research project in which context this thesis was developed with a famous Laurisilva forest spot in Madeira Island. In the following sections we explain our motivation for the study and describe how the eco-feedback was designed to address our goals of exploring non traditional forms of eco-feedback. The final sub-sections of this chapter presents the evaluation, results and conclusions from this study.

\subsubsection{Motivation}

The general motivation for the shift in the eco-feedback paradigm in the SinaisFromFanal study originated from the experience gathered in previous deployments. The insights obtained from the studies, described in the previous sections, disclosed that families stopped using the eco-feedback system after a period of time. Therefore, we wanted to explore alternative approaches that could overcome the novelty effect. As we discussed in the introductory sections (section 2.1), we are following the assumption that this decrease in attention to the eco-feedback system is ultimately responsible for the long-term lack of effectiveness of the eco-feedback.

In designing a new form of eco-feedback, we sought guidance from two distinct, but highly related paths of research. Firstly, we looked at work that presented the energy consumption as the impact on the environment and secondly we learned from studies that investigated how individuals perceive the effects of energy consumption in the environment.

Motivated by the promising results reported in the literature, when presenting consumption as the impact on the environment (see section 3.5.3 of the literature review) we gathered inspiration from art and nature, brainstorming ideas to change our strategy and implement a new eco-feedback mechanism.

This work also was motivated from the work of Spence et al. (Spence et al., 2012) where through a series of studies the authors claim that location where the effect of energy consumption (i.e. climate change) happens influences how individuals feel about climate change. For instance, climate change will be seen as a much serious problem if a flood caused by the rising sea levels happens closer to where people live as opposed to the hypothesis of this happening in the other side 
of the planet. This way the authors note that "making climate change relevant to the audience's locality and social groups is important in promoting action, as is reducing uncertainty over whether climate change is happening and reducing the perceived temporal distance of effects". This observation also relates to Spence et al. earlier work (Spence and Pidgeon, 2010), a study where 161 participants demonstrated that the perceived impact of climate change to be more serious at distant locations.

\subsubsection{Beyond Eco-Feedback}

We postulated investigating topics related to more inspiring and emotional visualization towards a less information-driven eco-feedback system. After brainstorming sessions with artists, designers and engineers we opted to design and implement the eco-feedback based on mapping the energy consumption of the household to elements of the local landscape, with the goal of leveraging the emotional connection of families with local natural heritage, i.e. the home of a UNESCO heritage laurissilva forest (see Figure 22). It is important to note that most of the inhabitants already feel a strong connection with the local forest. This was clear after several severe weather phenomena like the mudslides in 2010 and the forest fires in $2011^{8}$.
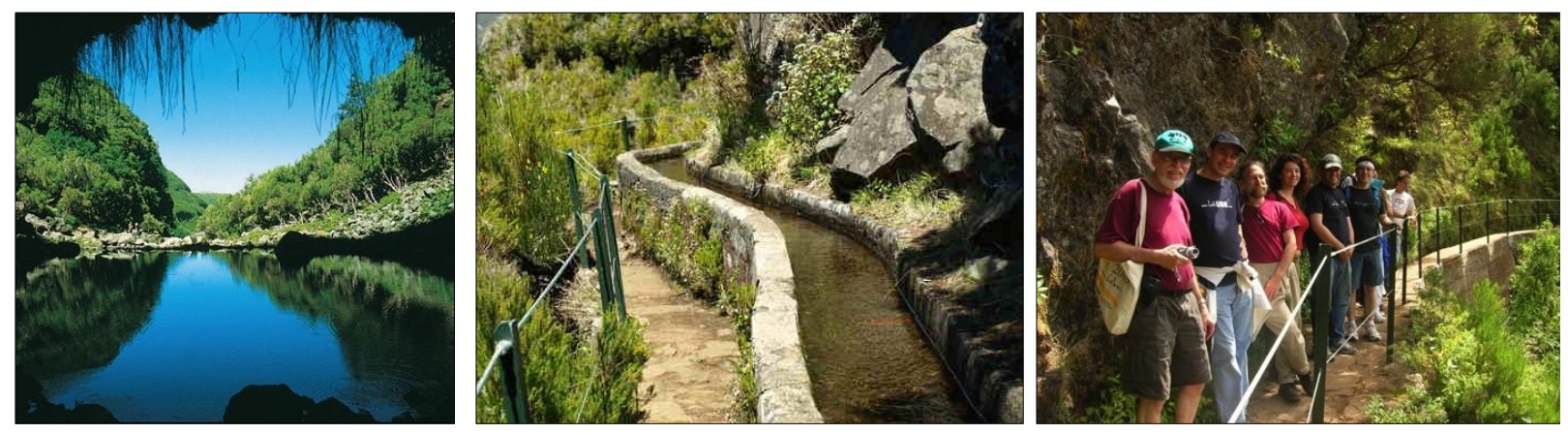

Figure 22: Madeira Laurisilva landscape.

${ }^{8} \mathrm{http}: / / w w w . r e u t e r s . c o m / a r t i c l e / 2010 / 02 / 22 /$ us-portugal-madeira-floods-idUSTRE61J2ME20100222, http://uk.reuters.com/article/2012/07/19/portugal-fires-idUKL6E8IJHXO20120719 


\subsubsection{Visualization - Mapping electricity consumption to the local forest}

To test the concepts described above, a novel interface was developed for the eco-feedback system. Two artists, a filmmaker and a photographer, spent approximately one-week time capturing the natural beauty of the island and exploring several concepts as to how it could be used to provide feedback. This version of the system was called SinaisFromFanal, SINAIS is the acronym of the project in which this research was involved. It also means 'signs' in Portuguese, we emphasize on the signs are sent from the Fanal Laurisilva site to the families through the eco-feedback visualization.

One challenge designing the SinaisFromFanal eco-feedback system relates to the fact that negative feedback fosters an immediate reaction in users after exposure. However, this reaction is known to last for a short period of time and fails to promote long-term behaviour change (Bénabou and Tirole, 2003). Furthermore, research has repeatedly demonstrated that negative feedback is not a suitable method to foster behaviour change (electricity consumption behaviour in this case)(Consolvo et al., 2009; He et al., 2010).

Our goal for the SinaisFromFanal eco-feedback design was to map the energy consumption to the natural landscape of the Island, without inferring judgment upon the user's consumption. In the following we explain several key design elements explored in this eco-feedback visualization.

\subsubsection{Fostering curiosity through the fog effect}

The very specific landscape of Madeira creates several particular weather phenomena, one of these is the sudden emergence of fog that completely hides the surroundings for moments, wrapping the trees and animals present in the landscape. This weather phenomenon happens at a certain altitude and quite frequently (mostly at the end of the afternoon) Madeira inhabitants clearly identify it as a local phenomenon. The thick white curtain can disappear as rapidly when the wind blows.

We decided to use this phenomenon in the idle mode of the application of the eco-feedback to hide part of the feedback, aiming at increasing users' curiosity about the information being displayed behind the white fog. For this reason, the default mode of the visualization the Laurisilva landscape is covered with fog. Once the camera detects movement in front of the screen the fog starts to decrease. If a person stands in front of the screen for more than a few seconds the system detects, using face detection algorithm, that the user is looking at it, hence is interested in the content. The fog then starts disappearing and leaves space to a landscape (Figure 24 top) that displays the current state of the energy consumption (described in the detail in the following section). This way, we 
aimed at encouraging people curiosity in finding out what lies behind the thick white layer of fog, and check the eco-feedback more frequently, by avoiding having the data exposed all the time.

\subsubsection{Expressing consumption with moving clouds}

During the conceptualization of the SinaisFromFanal system a series of time lapses were created. These time lapses depicted moving clouds and shadows of the trees, which besides capturing the beauty of the landscape added movement to the visualization. The eco-feedback uses these artefacts to map the real-time household energy consumption to a faster or slower frame-rate of the time-lapse, generating a mesmerizing visualization of a sea of clouds continuously moving in the sky just below the mountains where the Laurisilva thrives (Figure 24 middle).

The resulting landscape media visualization can attract attention to both high and low consumption mapping, fostering awareness without inferring judgment. With this kind of mapping awareness about nature and its beauty is the message itself, in this way avoiding to convey negative feedback

\subsubsection{Mapping appliances to elements of the forest}

Our sensor infrastructure enables a low-cost detection of electricity appliances (section 4.2.3 presents details about our infrastructure capable of detecting sudden changes in the consumption). The system is capable of detecting power on/off events and also classifies those events at the appliance level (e.g. when the microwave was turned on and how much energy was consumed by that particular appliance). All of this information enables various possibilities in terms of the detail that can be provided via the eco-feedback visualization and feedback per appliance or group of appliances (kitchen, living room, etc.). We proposed to map the energy events and activities to the already complex, but yet very natural, elements of the forest and trees (see Figure 23 and Figure 24 bottom).

In the final deployed prototype, we opted to map the power events of different power levels to: i) flowers blooming in the landscape for low consumption events and ii) animals for medium to high consumption events. Figure 23 (bottom) represents this mapping in action on the SINAIS platform. 


\subsubsection{Interaction with the SinaisFromFanal eco-feedback device}

The transitions between the three feedback strategies (fostering curiosity through the fog, expressing consumption with moving clouds and mapping appliances to elements of the forest), were made using the three levels of engagement on the eco-feedback device we used in the previous deployments (hidden mode, attract mode and active mode), described in 5.3.3.3.2. Next we explain how each mode was adapted to the SinaisFromFanal system, the transition between the different states are also represented in Figure 24:

1. If the system does not detect any movement next to the netBook for two minutes the depicted landscape would be surrounded by fog hiding it. With this animation that mimics a common phenomenon in the landscape we wanted to keep users curious about their consumption by hiding the information at the first glance.

2. Once there was movement next to the system the fog would gradually disappear and the feedback showed a landscape in Fanal where the real time consumption was mapped as the movement of the clouds, in the background of the forest (Figure 24 top).

3. If the user actually clicked on the forest the system would show a different part of the Fanal forest, and in this mode the electric appliances were displayed as animals or flowers that would appear and disappear (Figure 24 bottom).

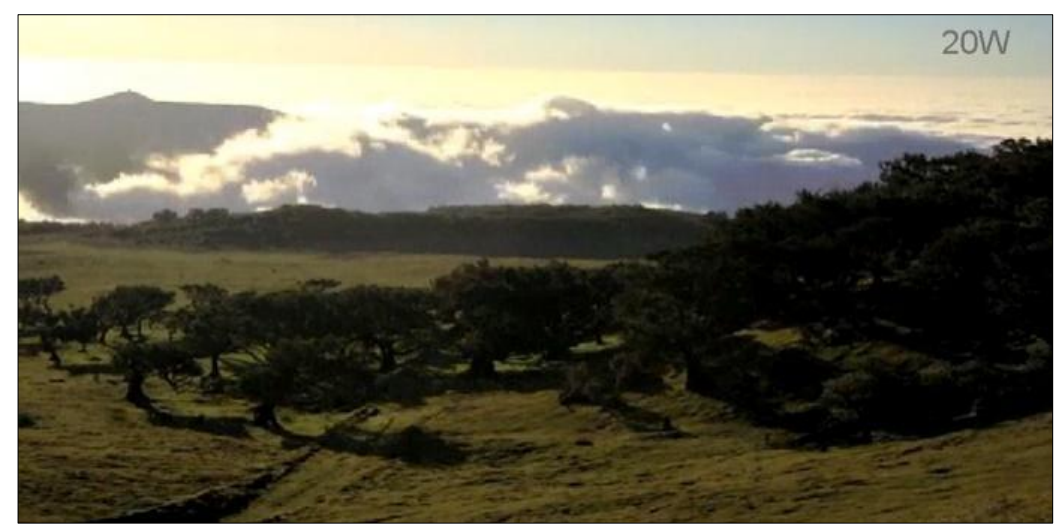




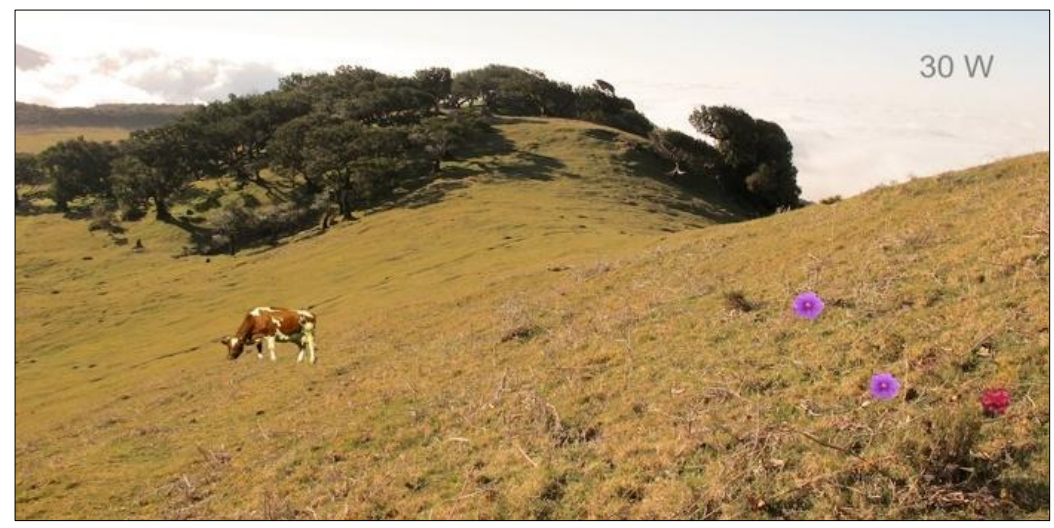

Figure 23: Screenshot of the application used in the SinaisFromFanal study. On top there's the main view of the forest, the consumption is mapped as the movement of the clouds in the background. At the bottom there is the landscape with elements added based on the usage of appliances.

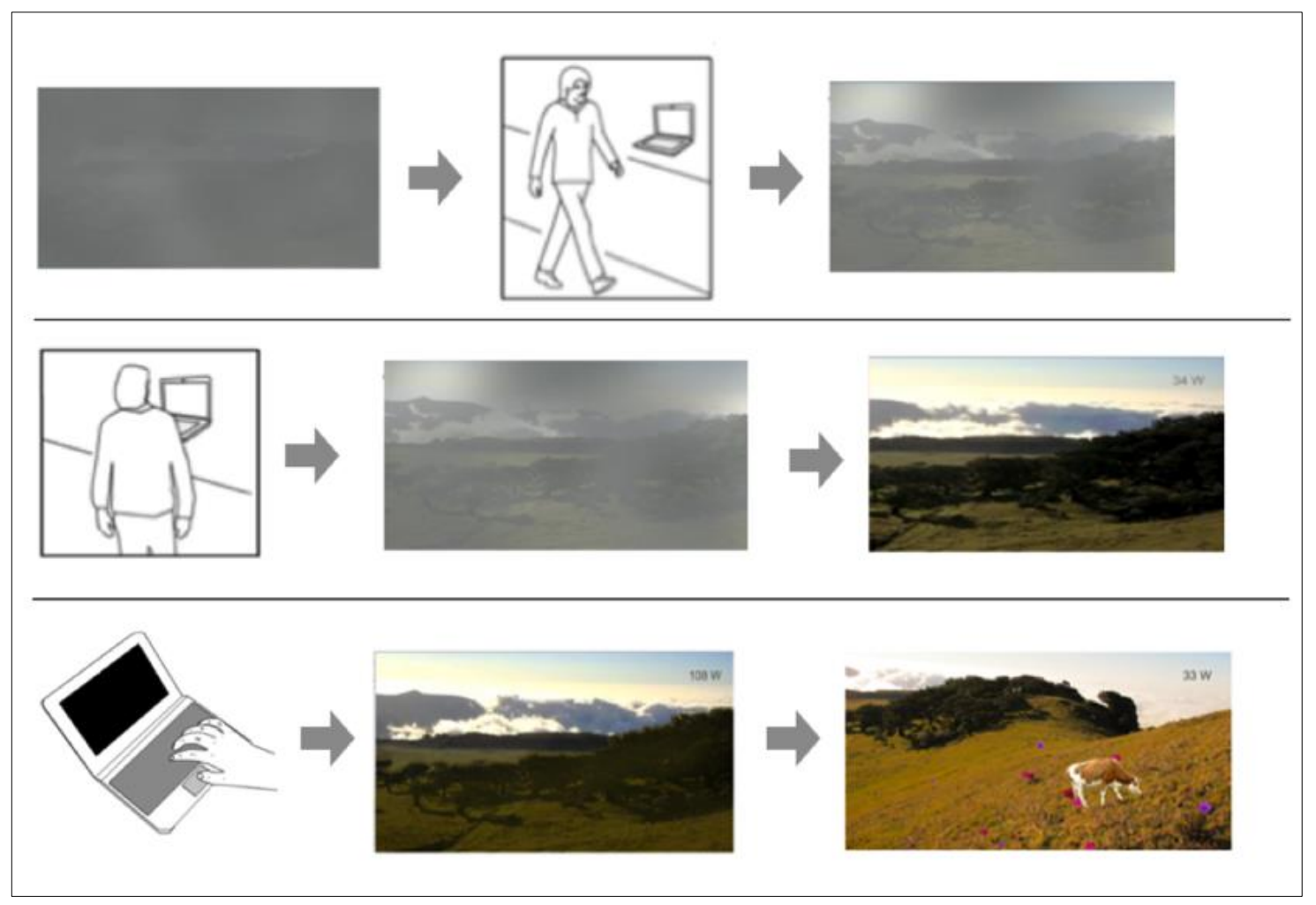

Figure 24: Different levels of engagement of users with the SinaisFromFanal eco-feedback system and corresponding response.

\subsubsection{Evaluation}

The system was tested in eight households over a period of one month and the selected families were part of the set of users of the two previous feedback systems (section 4.3.2 and 4.3.3). 
The selected sample for this study consists of 8 houses that were selected using the following method: The households were organized among 50\% percentiles for energy consumption (low consumption / high consumption) and 33\% percentiles for interaction with the system (low, medium and high interaction). To select a significant sub sample, the goal was to choose 2 houses from each group (totalling 12 houses). However, issues such network coverage, and other technical problems limited the number of participants. Also some families opted not to participate in this last study. Therefore, the low consumption/high interaction and high consumption/low interaction group had to be excluded, thus the final sample is composed of 8 families. In this analysis families will be referred as F1, F2, F3 etc. Consumption and interaction data were saved and four of the eight families were interviewed. The update of the system followed the same process as in the previous update (from version 1.0 to version 2.0), families were informed by phone that their electricity meter had been remotely updated, no further information was disclosed (for example we did not explained how the electricity consumption was mapped to the landscape). This way we wanted to test how intuitive the visualization was, and what would have been the instinctive reaction of the users to the visualization.

\subsubsection{Quantitative assessment.}

After four weeks of the deployment we aggregated all the databases from all the households in order to check if the frequency or extent of engagement of the families with the system had undergone any variation.

Figure 25 Shows that the new version of the feedback raised users' awareness for the system. However, if we look at the long-term effect (after the above mentioned 4 weeks) of this deployment, there is a noticeable drop in the in interaction between weeks 3 and 5 (consistent with our previous findings about the general users' relapse, see section 3.6 for the effectiveness of feedback over time). A qualitative interview from the research team with the families explaining the eco-feedback mapping in details, triggered renewed interest in the system and the interaction values peaked. Those values decrease in the next seven weeks with a similar ratio as before the interview.

Finally, we investigated how families that were not in the study behaved in the same period. For this we selected three families (one for each percentile of the houses with the new version). In total this houses had 34 interactions with the system over the same period as the SinaisFromFanal families study (four weeks' period). On average each house had 11 interactions ( $M=11, S D=8)$. The houses in the study had 6520 accesses, averaging 1 086per house $(\mathrm{M}=1086, \mathrm{SD}=1903)$. Which means that in average houses with the SinaisFromFanal prototype installed had 110 times more 
interaction than the others. We conclude that even though the novelty effect was present the SinaisFromFanal system raise users' awareness to the feedback device.

The installation of the new version of the system triggered in average less interactions when compared with the previous deployment. Despite that, houses with the new system had more interaction than the other ones still used the older system during the same period. Like expected the response-relapse pattern was observed. However, it was noticeable that 5 weeks after the installation the interactions increased abruptly. No change in the overall consumption was observed. Even if it remains clear that the main goal of any eco-feedback system is to reduce energy consumption, nevertheless the focus of this assessment was understand in depth how users interact with the ecofeedback system, and in particular one which is more inspired to aesthetic values and intuitive visualization, instead of a more abstract one, provided with charts and numbered values.

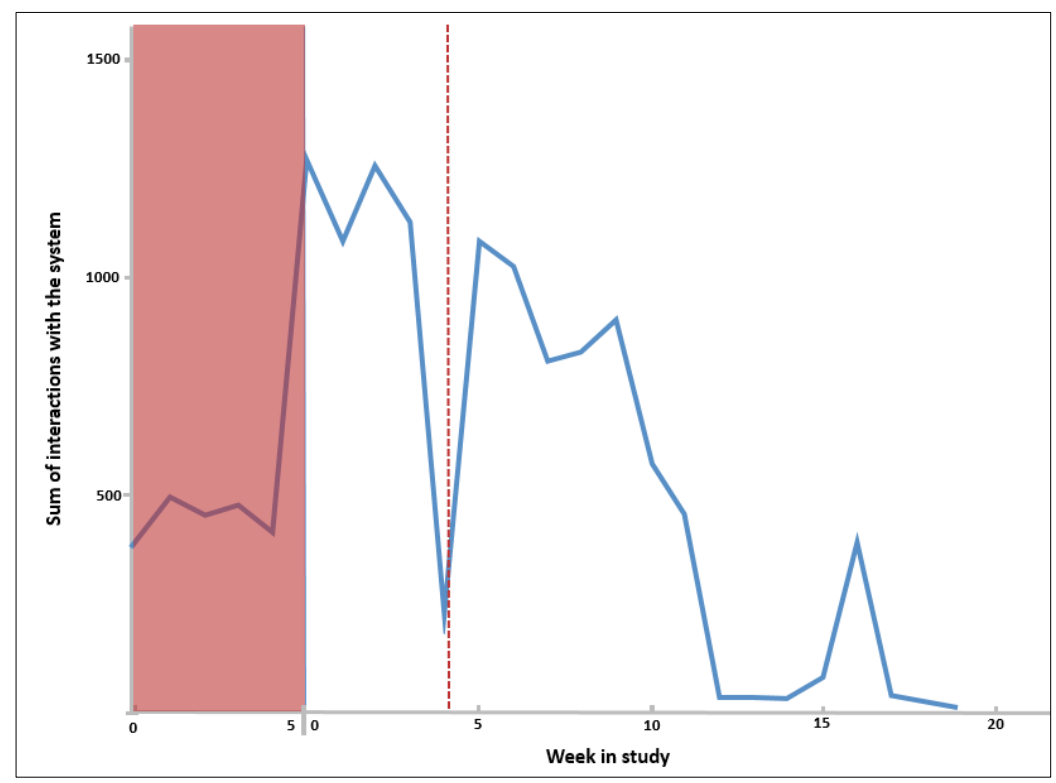

Figure 25: Sum of the weekly interaction with system before (red) and after the SinaisFromFanal study, the dotted line represents the date of family interviews.

\subsubsection{Qualitative assessment}

In order to understand how families interacted and reacted to the new system in depth, we conducted an extensive qualitative study and analysis of the users. We interviewed four out of the eight families exposed to the new eco-feedback. For simplicity we call these families F1 to F4, and specify their role inside the family. They all had children except one (F3). The children were not interviewed but sometimes cited by their parents. Parent's ages were between 30 and 47 years old $(M=41)$ and children ages were between 8 and 16 years old $(M=12,3)$. The semi-structured 
interviews lasted around 30 minutes per family. Interviews were transcribed and analysed extracting recurring and relevant emerging themes and grouping them under three main categories: emotional attachment (EA), information visualization (IV) and attitude or behavioural change (BC). We will describe the collected and analysed interview data below.

Out of the 4 families interviewed, two families (F3 and F4) recognized the landscape as local, by naming it: "It's Fanal" (F3, F4). The other two family members (F1 and F2) mentioned not recognizing the landscape because not paying attention to the visualization as shared by the mother in F2:

"we didn't pay enough attention to it" (F2, mother)

The mother from F2 also clarifies they were very busy lately and that:

"This last month we were not so available to look at the system" (F2, mother).

Moreover, the father from F1 mentioned that the details in the visualization were not enough in order to be recognized:

"I think we need to see the landscape with more detail" (F1, father).

We also found that families interpreted the visualization in diverse ways. Families F1 and F4, mistook the change to the new visualization for a technical problem:

"At some point I thought it was broken" (F1, mother).

Others also mentioned technical misconceptions "I thought the program was in stand-by" (F4, father). This made them (F1, F3, F4) keep coming back to the system to check it, as stated by this the mother from F4 'that's what I thought too. I thought you were working on something behind it" (F4, mother). One user thought the visualization was a screensaver (F4, wife).

Some family members (F1, F3, F4) were not affected by the positive feedback given by the visualization and would have liked to see more dramatic changes if consumption was rising beyond acceptable levels

"I assume that if there were more plants and animals, I was doing good things for the environment".(F1, F3, F4)

On the other hand, if the effects were tailored to negative feedback users might have got a better connection with their consumption behaviour: as the husband from F3 says: 
"... and if the consumption increases the landscape will probably show desertification" (F3, husband).

All families felt the previous visualization provided them more information, was clearer and precise, and allowed comparisons, but did find value in this one and described it as more simple and aesthetically powerful but more difficult to make sense of, as confirmed by the following statement:

"This one is simpler, there's just the image and the consumption on the right. The other one I could see the consumption in terms of the whole day and this was more elucidative. I find this one more interesting but we need some kind of heads up about how can see the consumption" (F3, husband).

Only one family definitely preferred the previous version:

"I preferred how it was before it presented the information using colors. It was more elucidative than this one. It was more attractive for us to look at it and could easily call our attention. Even for the kids, they would look at the other one more than this one" (F2, mother).

Families (F2, F3, F4) felt this latest visualization was incomplete in terms of the information provided but still somehow perceived the value of a more qualitative feedback, such as the mother from F4 says:

"I can see there is a value there. But before this I could see the consumption per hour, per day and now there is nothing like that. I don't have a way to see the highest consumption moments or peaks throughout the day. I miss it, it's like the speedometer, and it's like I have been riding for this amount of time and here's the total so far" (F4, mother).

As a result, families $(\mathrm{F} 3, \mathrm{~F} 4)$ felt they reduced their interaction with the system "I would check the other almost everyday day. This one I didn't check it as often. Now that I think about it I didn't do it because there wasn't so much data as the previous one" (F3, husband)

"I miss the graphs and the information as clear as possible. I checked the other one, almost everyday several times a day. I confess that now I rarely look at it" (F4, mother). 
But to note is that one family relates their lack of interaction with the new visualization to their decreasing motivation concerning general engagement with the whole eco-feedback trial, as shared by this mother (F4):

"We lost the interest in the device after the summer, and I feel this type of visualization might have made more sense in the first phase when we were motivated to learn about our energy consumption".

Nonetheless, family members (F1, F3, F4) found the visualization to be of value and pleasant, therefore, they expressed clearly that they would maintain it if additional information was integrated as suggested:

"This one is more interesting but I feel there could be more information at the end of the day of the whole day on the lateral side of the landscape. So I could see the historic data of my consumption, see the consumption throughout the whole month, the whole week" (F3, husband).

It is also important to consider that families had engaged with the system prior to the SinaisFromFanal visualization for several months before this study. They became used to checking their energy consumption through the previous visualization. The numerical and clear information format contained in the previous visualization was explained to them in details in order to understand their energy consumption. In fact, about this new system the mother from F4 shares:

"I think it is interesting having a landscape that changes according to our consumption and how much we use, however I need to have the data and the numbers" (F4, mother)

The father from F1 goes further and suggests: "I guess it could look like it is right now but it could be completed with the hour, day information " (F1, father).

Most of the problems that the family members encountered with the system and why they failed to relate their energy consumption with the visualization was due to lack of an initial clear explanation about the interface mapping and changes:

"I noticed some changes in the image, but I feel there were minor, and unless I was there for some time I would not notice them (...)" (F2, mother). Furthermore, she adds: "This last month we were not so available to look at the system to have the amount of time needed to really understand the changes in the landscape" (F2, mother). 
Due to lack of clear information delivery the feedback visualization could easily be misinterpreted:

"I couldn't relate it to my consumption. I assumed if there were greener fields and more plants and animals it was positive thing for me as I was doing good things for the environment" (F3, husband).

Some users were focused on finding elements from the previous visualization or even confused:

“No I didn't relate it to my consumption levels. I wasn't even near to realize that (laughs). I was looking for data, logical data about it (...) I could see some extra elements, I thought you were decorating the landscape but I didn't understand why were they being placed there" (F4, mother).

Overall, after the interviews it was clear that families exposed to this visualization seem to express a low intuitive understanding of the mapping but still connect to the aesthetic of the visualization portraying a natural landscape. The main issue raised by the users was that this visualization didn't accomplish its task of expressing the monitoring of the consumption as clearly as the previous one. Nevertheless, only one family really stopped engaging with it:

"For me it makes no sense being there anymore. It stopped being useful for me. I was always expecting you would add data to it and not just the landscape" (F4, mother).

All the other families thought this visualization was interesting (visually) and innovative, although it did not have the clarity of the previous visualization about their consumption, to which they got used to see through the previous deployment. These last families propose to have both ecofeedback systems working, as the landscape was more pleasurable to watch and the other one more informative in the details of their consumption:

"I think both are valid. Maybe there could be a symbiosis between the two. This one is more pleasant the other one is just data. What matters to me is to have the data, it's probably the most important for me. However, I like the way this one is presented, I think it's more interactive than the other one" (F3, husband).

Families (F1, F3 and F4) suggested the two visualizations could be merged to provide the information they need as well as the aesthetic pleasure of the natural landscape: 
"I like the landscape, and the place it is. I find it interesting you used it. But I feel like you need to be straight with people, have clear information, you are consuming this much, you need to change this and that. Maybe there is a way of combining these two concepts, data and a landscape. I am not sure how. Maybe the grass could become greener as you consume less or redder as you increase consumption" (F4, mother).

Another suggestion would be to divide the information per visualization:

"maybe you could use this one to show the day, and the other one to show the week and month information" (F1, father).

The multiple suggestions of how to keep the natural landscape interface and merge it with the quantitative previous one shows clear general interest and engagement with the SinaisFromFanal eco-feedback and points out clear directions for future work and improvement.

\subsubsection{Discussion}

By coupling Art practices and $\mathrm{HCI}$ we have stimulated a reflection that goes beyond traditional eco-feedback and tries to tackle important problems with this area of research, in particular the function of aesthesis in motivating people and fostering intrinsic motivations to act according to our ideals and values. Concretely, our research with the SinaisFromFanal system uncover three main findings.

Firstly, there were families which not recognized the landscape used in the eco-feedback, therefore if a system aims at connecting with consumers taking advantage of something close to them (in this case the Fanal landscape), consumers should be able to identify it for this design choice to be effective.

Secondly, the decisions of presenting the feedback in the forest in a neutral way, created confusion within users, families interpreted the information differently (two families even though the system was broken). This leads us to the third finding, the misperception presented above was only solved once the research team explained the presentation (how the consumption was mapped in the forest) to families. Considering the findings described above, if a system deploys neutral feedback for energy consumption, it should firstly make sure it is as clear as possible (so all users receive the same information), secondly if, even so, users need help interpreting the information the system should be able to aid them. 
Our last finding relates to the power of the aesthetics of the presentation deployed in SinaisFromFanal, although the system missed some information present in the older versions of the eco-feedback (section 4.3.2 and 4.3.3) families still liked the presentation used in the SinaisFromFanal eco-feedback system. This means that the aesthetic pleasantness of the system could be used to first lure families/individuals into using the eco-feedback system

This work proposes eco-feedback to go beyond a window for energy consumption into a mirror of our goals to live and act sustainably with an increasing emotional connection between our daily actions and the long-term effects in the natural environment. The study described here raised many important findings and critiques on a new form of eco-feedback. In the next sections we discuss further the implications of the findings described above. In order to facilitate the understanding, we group the discussion in three categories emerging from our analysis: emotional attachment, information visualization and attitude or behavioural change

\subsubsection{Emotional attachment}

From our study we learned that leveraging on the emotional attachment of the eco-feedback users with elements of the natural environment is a potentially powerful way to raise people's sustained awareness for their energy consumption. The qualitative assessment confirmed that people appreciate this type of mapping and the quantitative data confirms increased interaction with the ecofeedback system. However, some major issues have risen regarding the choice of the landscape, not always recognized as local, and the meaning of the mapping of the energy to the visualization. Our findings suggest that additional care should be taken when choosing the natural elements to make the emotional connection more clear. A potential improvement would be to test or work with the users themselves and choose together the natural landscapes or images that could guarantee a more effective emotional connection. As it stands in fact the system is hidden, usually situated nearby the sensors for the electricity and hence far from being visible in the commonly inhabited spaces of the house, while most of the users found the visualization aesthetically pleasant. Position the visualization in a more prominent place, such as on a tablet, in the living room for example, or in a commonly used space, could expose the families to more frequent feedback, hence leading to more awareness of their consumption habits.

\subsubsection{Information Visualization}

The choice and the mapping of the consumption to the visualization also provides an important challenge. The families did not intuitively perceive some of the visualizations suggested by the artists 
and implemented by the research team. For instance, the mapping of cloud speed of movement to the speed at which energy is consumed instantaneously was not clear to most of the users. Suggestions from the users point out that changes in the landscape and animation should be made clearer and explicit in their meaning regarding consumption of electricity- for instance changing the colour of the grass, from greener to dry would mean an increase of energy consumption. The mappings for disaggregating consumption (e.g. appliances, time periods, etc. mapped to the appearance of animals and flowers in the landscape) suffer from the same lack of clarity and should be further tested with end-users beforehand in order to improve the mapping and recognition. Furthermore, users themselves often suggested the employment of negative feedback as an effective way of mapping consumption to the landscape (if you consume more, the grass will dry, for example), which was something we wanted to avoid, due to lack of long term results, reported by previous studies. This result is in line with Froehlich observation in the evaluation of the Ubigreen mobile application, in which participants suggested that negative reinforcement could have been used in the design of the system (J. Froehlich et al., 2009b). Furthermore, our findings regarding the presentation of the consumption recalls Rodgers and Bartram observation relating the ambiguity in the feedback, although it can foster curiosity, it ultimately can make the comprehension harder and should be used together with concrete data (Rodgers and Bartram, 2011), this way our work provides further evidence of these statement.

\subsubsection{Behavioural Change}

Overall the results from our study show an increased awareness of the users about their consumption. This result was stimulated from the novelty effect (of having a different interface in an already known system) but also from the family discussion emerging from the not always clear mappings of consumption to the natural elements, this increase in awareness is in line with a big body of eco-feedback research. Our contribution here is another confirmation of an established assumption that one of the biggest outcomes of eco-feedback devices is an increase in consumer awareness ((Froehlich et al., 2010; Pierce et al., 2008) provide good summaries of eco-feedback research).

An important limitation of our study was the fact that SinaisFromFanal was installed in families that were already familiar with a quantitative and highly informative eco-feedback system. The previous system already provided detailed information about the consumption, and to substitute the visualization with the artistic based one, without a detailed explanations led to a lot of confusion. The artistic eco-feedback is not an alternative to the detailed information but rather a complement. Our findings support that combining both strategies would be optimal in increasing awareness and 
promoting behaviour change while also giving detailed information that could help users understand their consumption patterns.

\subsubsection{Conclusions}

The quantitative and qualitative evaluation of SinaisFromFanal during a period of one month in eight houses provided many insights on how people react to novel eco-feedback systems that map consumption to elements of the natural environment. On average houses with the SinaisFromFanal system installed showed increased interaction than the houses with traditional systems. Families felt the previous visualization provided them more clear information, although when asked they didn't want to give up the new visualization of the landscape but rather combine it with the previous more informative one. A potential solution to this problem would be to combine both solutions and make the artistic visualization the frontend. Most of the family members failed to relate their energy consumption with the visualization because of the subjective natures of the metaphors. Overall, families exposed to the new visualization seem to express a high aesthetic appreciation for the new system, which showed as they provided many insights on how to improve the visualization and seemed very engaged.

In summary this study provided evidence that artistic visualizations of energy consumption are well received by users as long as the mapping with the natural element is clear and detailed energy information is conveyed. 


\subsection{A nature-inspired portable eco-feedback device: WattsBurning}

This chapter describes the study performed with the WattsBurning eco-feedback system. With this system we aimed at combining the quantitative data from "traditional" feedback systems with the more abstract information presented with non-conventional eco-feedback systems such as the SinaisFromFanal described in the previous section (section 4.4). In the following we present our motivation, the design and implementation of the new system named WattsBurning. Lastly we describe how the system was evaluated and the results of a 17 week deployment.

\subsubsection{Motivation}

The experience gathered from the SinaisFromFanal (section 4.4) study, showed that users found the aesthetic qualities of the eco-feedback compelling, but they still wanted the concrete data about the consumption. Such findings are in line with a similar observation made by (Fitzpatrick and Smith, 2009). The mapping between the forest and consumption was not intuitive and most users needed further explanation as to how to interpret it. Additionally, it was also important to address some of the limitations found in the two first deployments (see section 4.3.2 and 4.3.3) in order to redesign the feedback system and amend its apparent flaws:

- In the initial three deployments, the feedback was given via the display of a small netBook installed behind the main door of the house (where the main fuse box is located in most of the houses). This type of stationary feedback is clearly limited since it is not accessible to all the household members at the time of decision (e.g. turning off a high consumption appliance).

- The feedback should be accessible to all family members since family dynamics and communication have an impact on the decision (Barreto et al., 2011). Furthermore, we were also motivated by Kjeldskov (Kjeldskov et al., 2012a) work which disclosed that the portability of the feedback device was one of the strengths of their Power Advisor application.

- The system was connected to the main fuse box worrying some families about the safety of the device (Pereira et al., 2012), and sometimes not allowing children to use it.

- The system should allow for the comparison between different periods (hour, day, weeks and months) so that people can explore and better understand their consumption patterns.

- The system providing simple tips about energy conservation and best practices promoting sustainable behaviour change was most appreciated. This was one of the features favoured by participants in the SINAIS Power Meter 2.0 system (see section4.3.3). 
Summarizing, the lessons learned from our experience we realized that our new eco-feedback version had to undergo major updates both on the software and hardware sides (in section 4.2.5 we discuss further the implementation of the multi-house sensing). The eco-feedback device should ideally be portable so the system can be accessed from anywhere in the house (or outside). The sensing framework should provide data remotely to the eco-feedback device so that the sensing is removed from the house, addressing the safety concerns of users.

\subsubsection{System design and Implementation}

Following the requirements described previously, we implemented a new hardware and software platform (see 5.2.5 for details on the hardware used in the WattsBurning study) but also modified the eco-feedback component, which now could be deployed on different portable devices.

\subsubsection{WattsBurning eco-feedback visualization}

The new visualization addressed the issues uncovered during the SinaisFromFanal study (section 4.4) producing an aesthetically pleasing landscape that mapped household consumption to the deterioration or improvements of a landscape snapshot of the local natural forest. In addition, it provided a way to access a second layer of more detailed consumption information. In this design we followed recommendations for a complete (Rodgers and Bartram, 2011) eco-feedback device that combines the appeal of an abstract representation with the concrete data consumers need in their daily routines.

This way the design of the WattsBurning eco-feedback visualization, like the SinaisFromFanal, acquire motivation from Spence's work (see section 4.4.1 for more about this work), by using the local landscape as means of feedback. However, users were included in the design of the WattsBurning, as they were asked to select a landscape emotionally close to them, and decide together with the designers how the individual consumption can affect the local landscape.

The customization of the feedback was motivated by Petkov et al. (Petkov et al., 2012) work where the authors shown that different users have different needs in terms of eco-feedback mechanisms. Furthermore Petkov et al. work also discloses several designed guidelines followed in the WattsBurning design such as : "An application should provide different kinds of feedback" WattsBurning accomplishes this by presenting the consumption information in different modalities such as charts, numerical values or abstract representation in the local forest; "the feedback provided should be localized and contextualized" - the WattsBurning forest animation contextualized and 
localizes the consumption information to users, "Applications should be designed to provide proximal feedback" the local forest representation also meets this guideline by presenting the feedback mapped in something closed and chosen by users themselves.

However, our work does not follow the specific recommendation of "Feedback should be framed in a positive or neutral way, rather than presenting the negative impact of users' energy consumption", since this guideline was already followed in the previous SinaisFromFanal study and was found flawed (see section 4.4.5). On the other hand, in the WattsBurning design we allowed users to select how the consumption would be mapped, therefore the mapping followed user's' preferences whether it was by means of positive or negative feedback.

The eco-feedback visualization was implemented on a 7" android tablet using the android native SDK (Figure 26). The tablet specifications allowed us to implement rich visualizations, which could be accessed in different areas of the house, or even outside. We believe that the device itself (a tablet) was still viewed as a novelty, and that this helped our application to fit into the household as well as encouraging participant interaction. The application received real-time consumption and historical data from the sensing framework. The real-time data was accessed using sockets and a custom-made communication protocol. Historical data was gathered using the web-services described in the previous sections. The historical data was also stored on the tablet so the users could check their past consumption without an Internet connection.

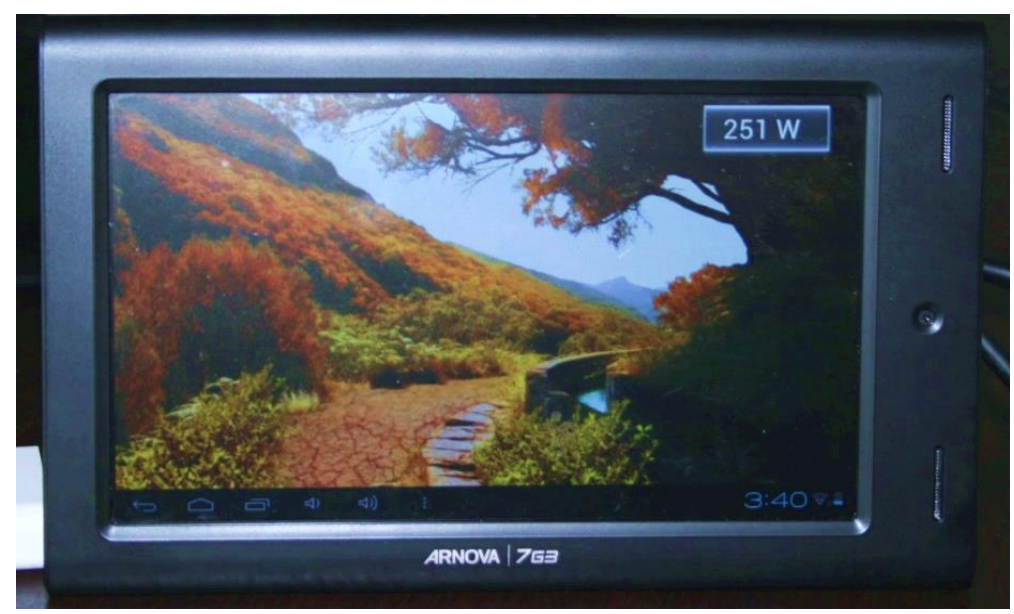

Figure 26: Hardware used for the WattsBurning eco-feedback system.

The transitions between the different modes of operation were stored locally enabling the analysis of the usage patterns. For example, if a user picks up the tablet, presses the back button to go to the home screen and selects the daily consumption, the application will store three interactions 
with three different IDs and timestamps referring to the three different views accessed by the user system installed in one of the households.

The eco-feedback system incorporates two main modes of operation. When it has not been used for two minutes the system goes into the Energy Awareness mode that shows the consumption mapped as a digital illustration of the local endemic forest following the design rationale explored in the SinaisFromFanal prototype. Once the user interacts with the tablet, by pressing the back soft key, the system goes to Detailed Consumption mode and shows daily, weekly and monthly information about the home energy use. It is important to point out that Energy Awareness was the default mode of the system, the tablet never went into idle mode as happens by default in some android applications.
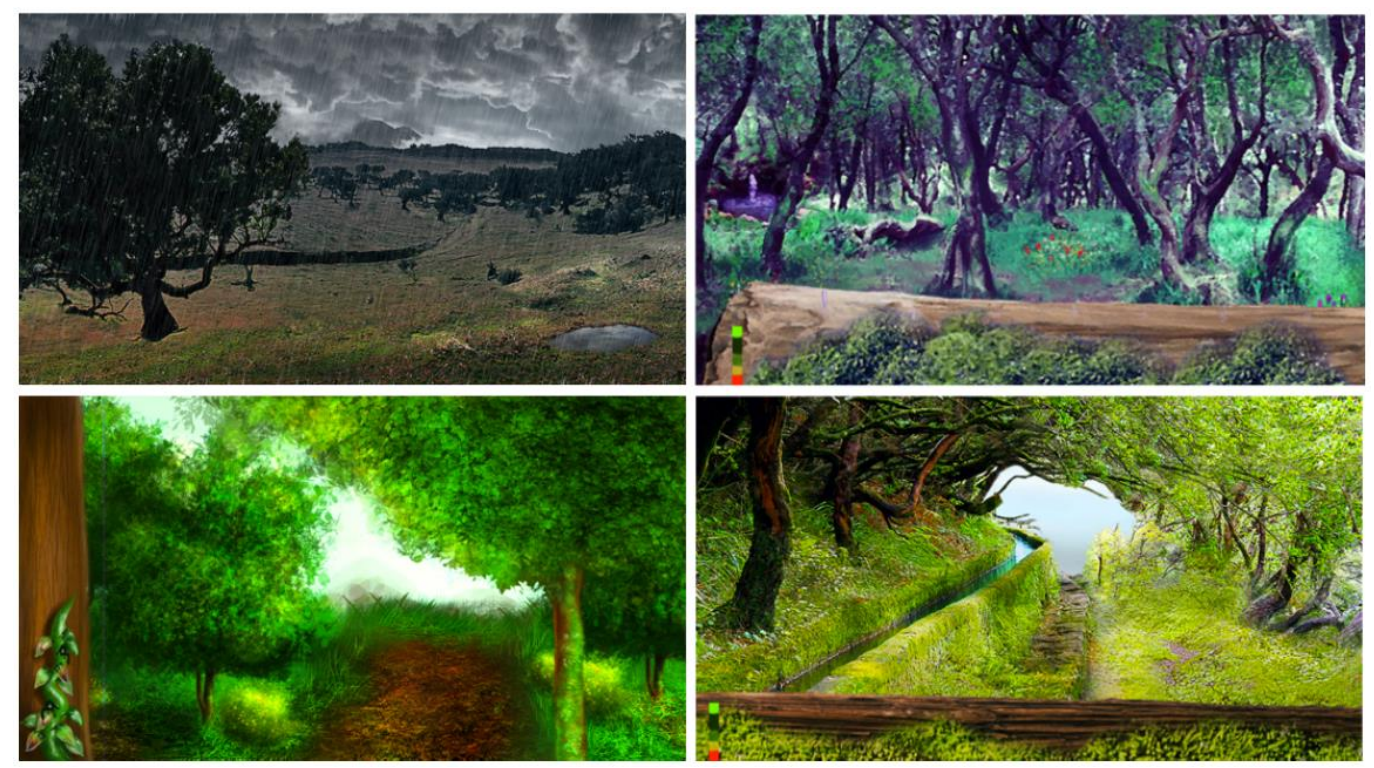

Figure 27: Different landscape visualizations developed by the involved artists and used during the thinkaloud process to include the users in the choice of their favourite landscape.

\subsubsection{Energy awareness mode.}

This feedback feature of the system is one of the innovative features that has not yet been tested in literature, specially using a local landscape that is clearly close to the consumer. Therefore, it was very important for us to evaluate how we could use the local landscape as means of feedback, considering the results from the SinaisFromFanal prototype (section 4.4.5), which indicated that a more noticeable/visible mapping was needed. 
To select the landscape and the metaphors used in this mode we performed a think-aloud session with two families (these families were later recruited as participants for this study). In these sessions the family members were presented with several paper prototypes displaying the consumption mapped onto different landscapes and represented with different items in the forest as seen in Figure 27.

We decided to use an image of a well-known forest site that was easily recognizable to the local community. The digitally modified pictures of the forest represented the comparison between real-time consumption and an average baseline consumption level. Two digital artist were recruited to help us in the process, they visited several landscapes in the island took pictures, brainstormed how the consumption could be mapped to those places, and produced several prototypes that were used in the think-aloud sessions.

After the think-aloud session some concepts such as displaying historical consumption as items in the landscape, for example moss growing on a tree (Figure 27 top right), the real-time consumption mapped as the movement of animals or mapping the consumption as the weather in the forest (Figure 27 top left) where rejected by the families as they were not clearly understood by the participants in the think-aloud session. In total we opted for five levels of consumption to be mapped onto the in the visualization of the local landscape: from an apocalyptic fire destroying the landscape in case of excessive consumption to a lush and thriving forest for low energy consumption levels (as shown in Figure 28). These five levels represent household consumption at slightly above/below average, well above/below average or belonging to the average baseline. The baseline was composed of an average of the consumption during that period, for example the real time consumption on a Monday at 12:20 was compared against an average of all the Mondays during the period between 12:00 and 13:00. Additional illustrations of the forest are used to ensure a smooth transition between the states, however the animation only stops at the five aforementioned levels. When in this mode of operation, the tablet resembles a digital frame holding a picture of the families' favourite place in the local forest (see Figure 26). This mode draws inspiration for the analysis from (Foster et al., 2010), where participants mentioned it would be interesting for an eco-feedback to function in this manner. 

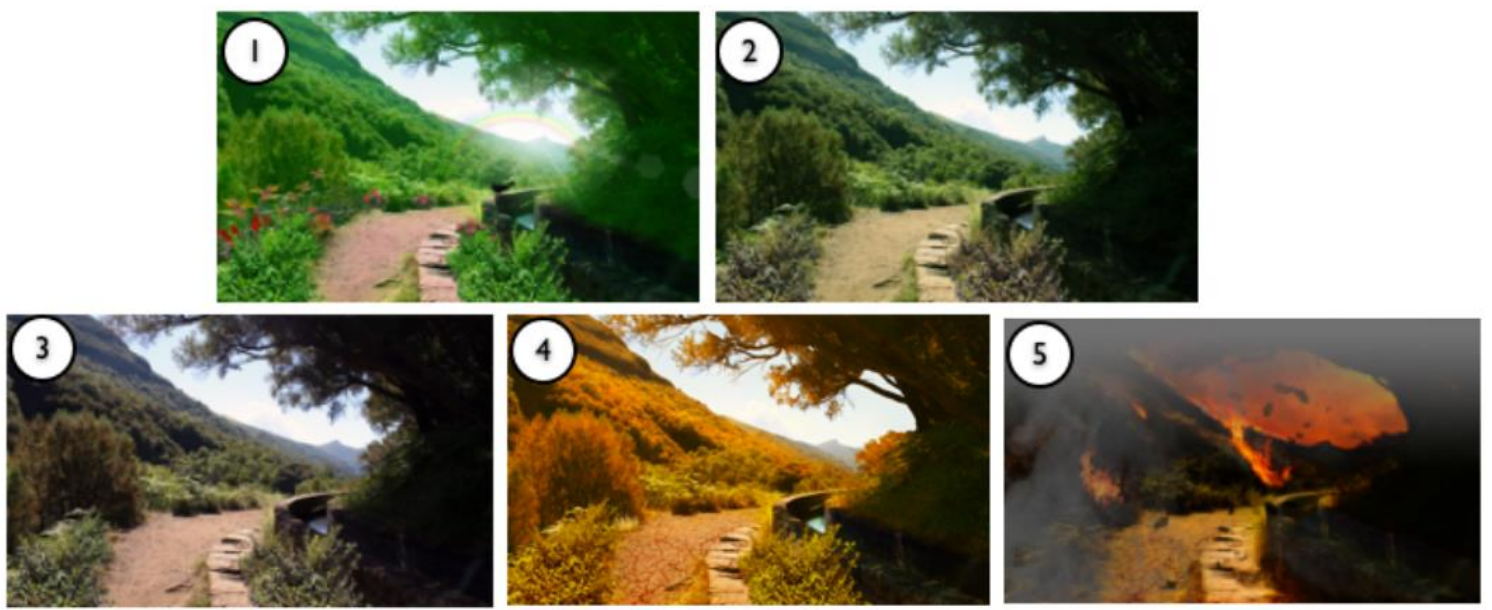

Figure 28: Different views of the landscape according to the consumption. Ranging from low consumption

(Image 1) to high consumption (image 5).

\subsubsection{Detailed consumption mode.}

This mode presents a more traditional representation composed of charts numbers and comparisons, and is triggered when the user presses the tablet back button. As a consequence, the system presents a tabbed menu with four options: "Home", "Day", "Week" and "Month". The "Home" tab shows a summary of the overall consumption as well as the current real-time consumption (Figure 29 Left). The summary contains aggregated consumption of the current day/week and month, and comparisons between homologous periods. Also in this tab the user is presented with a "tip of the day" with general sustainable actions. The "Day" "Week" and "Month" tabs (Figure 29 Right) present a chart displaying the consumption over that period and the total aggregated consumption. It also informs the user of where the peak consumption happened and how it compares to the average of that period (for example in the "Week" tab the system shows how the consumption in the current week compares with an average of the previous weeks). By default, the information presented here refers to the current day/week/month but the user can select preceding periods. 

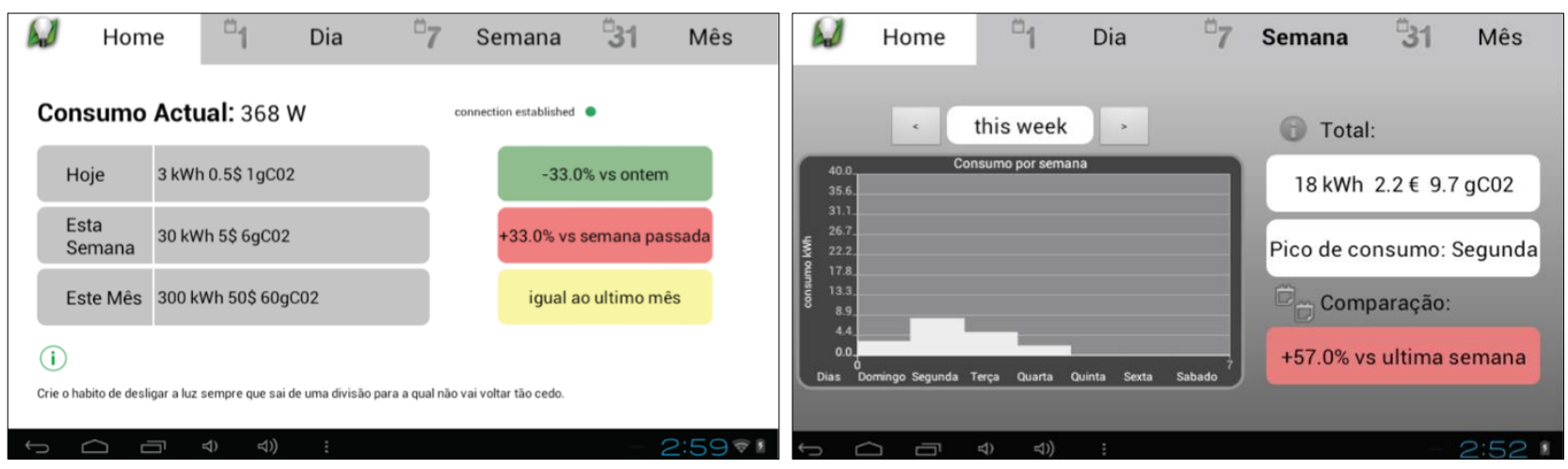

Figure 29: Left: Home screen of the system. Right: Tab with the consumption of the current week.

\subsubsection{Collected Data}

Our sensing framework samples the current and voltage waveforms at a high sampling rate $(3.2 \mathrm{kHz})$ but due to database size constraints only average power consumption is stored in the database (in 30-second sampling intervals). Additionally, the application stores locally every transition between the different modes of operation of the eco-feedback system which is then periodically uploaded to one of our servers, thereby allowing us to keep track of how the system is being used without having to wait for the end of the deployment.

The data analysed corresponded to two weeks of baseline consumption data collected when no eco-feedback was available and fifteen weeks of consumption data after the eco-feedback was installed, as well as all the user interactions with the feedback during that time.

\subsubsection{Evaluation}

To test our system, we recruited users from an apartment complex in an urban area in Funchal (section 4.1 contains a detailed description of the location). All the apartments were relatively new and they had similar appliances (provided by the construction company), consequently all of the families were relatively new to their houses. The sample is composed of six households: five couples with one or more children, and one young couple with no children. Two of the families had to leave the study earlier, but in both cases this was related to the fact that the families had to leave their apartments and not because they wanted to abandon the eco-feedback study. The families were told that they could use the tablet as they wanted (for example for browsing the web). But only the interactions with the WattsBurning system were recorded, as described in the previous section. 


\subsubsection{Method}

In order to assess the changes in consumption we collected baseline consumption data before the eco-feedback system was installed. This data was collected between the 13th and the 27th of August 2013. After that each family received a tablet with the WattsBurning application installed and given a short explanation of how to access the consumption data. After 22 days we visited the families and performed a short semi-structured interview. The families continued to use the system until the end of the year. Here we analyse the consumption and interaction data until the 17th of December for a total of 17 weeks. We wanted to avoid the Christmas period, since the results in that period would most probably be biased because of the difference in pattern activities of the families.

\subsubsection{Qualitative Assessment}

When the families were interviewed after the first three weeks, we wanted to understand how the system was received, and if the Energy Awareness mode was being clearly understood and how it was impacting families' awareness of energy consumption. It was also important to verify if any behavioural change was triggered by the presence of the eco-feedback system. Five of the six families were interviewed, they will be referred to as F1, F2, F3, F4, and F5. We asked all the family members to be present at the interviews, in order to gather opinions from every member of the family about the system. However, for F2 and F3 only the husbands were present during the interview. Appendix 9.3 presents the protocol followed during the WattsBurning qualitative evaluation.

The system was well-received by all the families, and none of them had any major problems using it.

"I think it was simple, even the wireless connection was stable" (F4, wife)

All the families agreed on the fact that the system increased their awareness of electricity consumption.

“It's raising my awareness, I don't think I've changed my consumption patterns yet, but I'm more aware now". (F1, husband)

"... if we have more devices turned on we can see right away there is an increase" (F2, husband) 
"It provides us with immediate feedback, such as daily and weekly consumption, and we can see what we do and how we behave in our daily routines to reduce our energy consumption" (F3, husband)

"I got more alert, so necessarily I will try to consume less" (F5, husband)

An observation transversal to all of the families was that every family learned something about the consumption of certain devices.

"In the weekend for example, we were using the oven and I noticed it consumed a lot, then I turned on other devices out of curiosity" (F1, husband)

"...the electric stove for example, I learned from the system that it consumed a lot" (F2, husband)

"Especially the oven, it increases to 2000/2500W... I see there that it goes to $200{ }^{\circ} \mathrm{C}$ but I wasn't expecting so many watts... I was even surprised with the TV's'" (F3, husband)

"I've learned that the oven consumes a lot, also the fridge" (F5, husband)

It was also important to verify if the Energy Awareness mode of the application was well understood. All families found that it was easy to relate their current consumption to the animation of the forest drying up and eventually being set alight. It was also mentioned that the picture showing the state of the forest in the eco-feedback interface worked as an alert as to what was going on in the house, and it made them more aware of their consumption at specific moments.

"Yes but.... For example when the dryer is working I see the forest catches fire, but I was expecting that, but I knew I couldn't keep that consumption for a long time, maybe I got more conscious" (F1, husband)

"It gets dryer until it catches fires, when I have a lot of things turned on (...) also when there were only a few things on it showed rainbows and butterflies" (F2 ,husband)

"(...) there's an association, the bigger the consumption the more destruction is visible in the forest, when we see it goes from green to yellow to red,... it's scary, " (F3, husband).

All the families opted to place the system in a central location in the house, where it could be visible to all the family members. However, of the five families that were interviewed it was mostly the adult male and young children who used it more often. 
"... I was curious in the beginning to see the forest on fire, but it was mostly my kid" (F2, husband)

"It was mostly me and my son, my wife and my daughter didn't pay much attention to it, it was me and my younger son" (F3, husband)

"It was mostly me, she (girlfriend) wouldn't use the system". (F5, husband)

Although the system was usually located in a central, visible place in the house, it was common for participants to move it around, mostly because certain families wanted to check the consumption of a device in real time.

"It was mostly there, but it was in the kitchen for some time" (F2, husband)

"It was there close to the sofa, because it had better reception for the internet (...) but I would also take it to the balcony while sitting there" (F3, husband)

"I used it in different places in the house, I thought of it as one of those portable weather forecast displays that can be placed anywhere". (F5, husband)

\subsubsection{Quantitative Assessment}

From the qualitative assessment users reported a noticeable increase in their energy awareness. However, it is important to confirm if the reported awareness translates into an actual behaviour change, confirmed by the quantitative data acquired from our sensing platform. In the following sections we analyse and discuss this topic.

\subsection{Energy consumption}

In order to compare energy consumption, we use a week as the standard period of time. This is the unit that best spans the family routines impacting the energy consumption. For instance, some families organize the major cleaning on a single day of the week, others the ironing. The week also comprises the working days and the weekend that usually correspond to very different routines. Therefore, we compare the consumption data in the 15 weeks of eco-feedback deployment with the average of the two weeks of baseline data.

If we consider the 6 households as a whole, after 15 weeks the average weekly consumption dropped on average $2 \%$ from the initial baseline $(\mathrm{M}=0.02, \mathrm{SD}=7.99)$. However, the standard 
deviation suggests that the savings were not constant across the houses. As a consequence, we analysed each house individually. Three families (F1, F2, and F5) reduced their consumption by $5 \%$, $7 \%$ and $13 \%$ and their consumption was below the baseline for long periods -12 weeks for F1, 11 weeks for F2 and 5 weeks for F5. The other three families consumed, on average, more than the baseline, respectively $10 \%(\mathrm{~F} 3), 2 \%(\mathrm{~F} 4)$ and $6 \%(\mathrm{~F} 6)$. These 3 houses were below the baseline average for 3, 6 and 6 weeks respectively. Figure 30 shows how each family's consumption changed during the study.

We investigated the consumption through the study further by performing several statistical tests, which allowed us to compare consumption differences between different houses and periods

To verify significant differences between the baseline and study periods the consumption data was averaged by week for each group. To that set of data a paired sampled t-test was applied which did not reveal significant differences between the two periods. Considering all houses one ANOVA test allowed us to check for differences between the households, this test was significant which confirmed our overall observation that different houses had different consumption patterns $[\mathrm{F}(5,54)=50, \mathrm{p}<0.001]$. Furthermore, a TukeyHSD test revealed that out of the 15 pair possible combination between households, 8 were significant, this result solidifies our conclusion that consumption between households were actually different $(\mathrm{p}<0.001)$.

For our next tests we considered the consumption as a function of time (week in the study) and applied linear regression models to the total consumption as well as to each individual household. Out of the 7 models created ( 1 for the total consumption plus one for each household) 4 were not significant (total, F1, F3 and F4) and three were significant (F2, F5, F6) with minor negative slopes, which indicate a reduction trend $(\beta=-.0 .024, \mathrm{t}(17)=-5.901, \mathrm{p}<0.012 ; \beta=-.0 .001, \mathrm{t}(17)=$ $-4.712, \mathrm{p}<0.001 ; \beta=-.0 .039, \mathrm{t}(17)=-7.008, \mathrm{p}<0.001)$.

One final test allowed us to compare if the consumption was different among different days of the week. The motivation of comparing consumption between different weekdays for differences is related to the possibility of tailoring different feedback/services to different weekdays. One ANOVA test returned a non-significant difference, which combined with 21 non-significant pairs from a TukeyHSD test confirms that no observable difference was found among weekdays (p>0.001). 

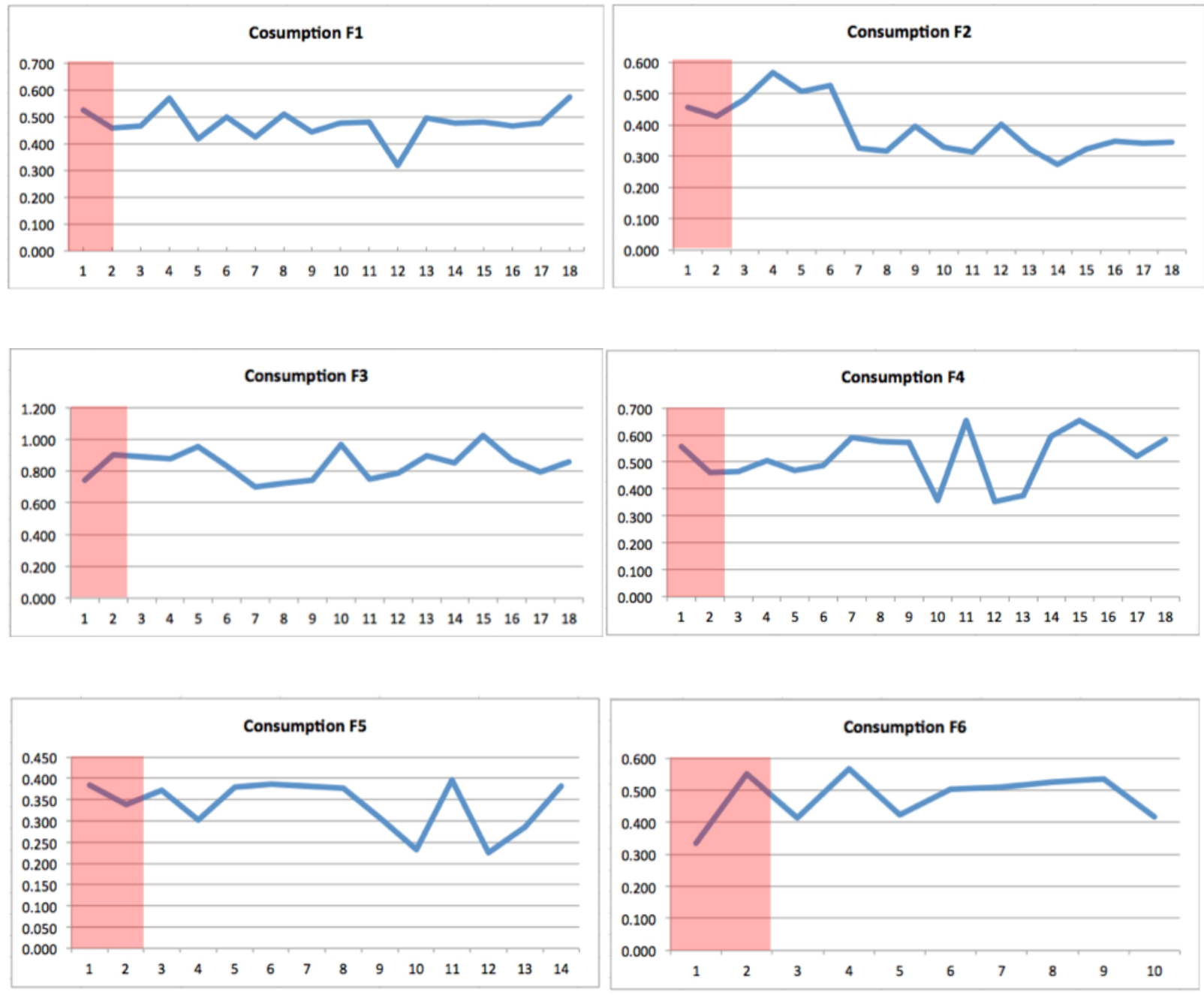

Figure 30: Consumption in the 6 households during the study. The baseline period is highlighted in red. The horizontal axis represents the week in the study, and the vertical represents the average consumption in $\mathrm{kWh}$.

\subsection{Interactions with the system}

The analysis of the logged user interactions showed that families used the system in completely different ways (the saved interactions are defined in section 4.5.4). Figure 31 displays how the interactions evolved during the study, each family is represented by a colored line. Two of the families (F3, F6) used the system more on the Mondays, while F1 and F4 used the system more on Wednesdays, F2 used the system mostly on Fridays and F1 on Tuesdays. This diversity was also noticeable when looking at how families used the system throughout the day. However, in all the families the view that was most triggered was the Home View, followed by the Energy Awareness view. 
During the 15 weeks of the study, a total of 2738 interactions with our system were logged across all of the households. This value represents an average of 456 interactions per house $(M=456$, $\mathrm{SD}=688)$ and an average of 29 interactions $(\mathrm{M}=24.4, \mathrm{SD}=29.4)$ per day and 4.2 interactions per house and per day $(M=4.2, S D=6.06)$. Our system was mostly used in the afternoon (between 13:00 and 19:00), almost a third of the interactions happened during this period. It was also noticeable that all the families had a lot of interactions in the first four to five weeks of the study. After that the number of interactions dropped significantly (by more than two thirds). Again the pattern was not the same in each house, which justified the high standard deviation. Table 3 shows a summary of the number of interactions with the system, in the first four weeks of the study, the rest of the period and in total. We chose to isolate the first four weeks because our previous research suggests that after this period there is a steep drop in the usage of the eco-feedback devices (Nunes et al., 2011; Pereira et al., 2013).

To better understand the usage patterns we analyse each household individually. The number of interactions in F1 only decreased by less than half, they kept using the system with an average of 14 interactions per day after the initial four-week period $(M=14, S D=12.4)$. Similarly, with $F 2$ the decrease in interaction was not considerable, but it was noticeable that they stopped using the system on a daily basis. Families 3, 4 and 5 had a lot of interaction with the system in the first week, after that the interaction values dropped to less than one interaction per day $(0.6,0.4$ and 0.2 per day respectively). Family 6 simply didn't interact directly with the system, even though this family agreed to participate in the study, they never showed much interest regarding the system nor they were ever available for interviewing. These observations show that houses used the system in different manners (both in terms of interaction patterns and frequency), to further test this observation, the interaction data was organized by house and subject to an ANOVA test which returned significant differences $[F(5,54)=12.76, p<0.001]$. A TukeyHSD test allowed us to compare the difference in the interaction pair-wise, out of the 15 possible combinations 5 where significant, and 4 for those where between F1 and the other families, which indicates that this family had a unique usage pattern $(\mathrm{p}<0.001)$.

The frequency of interactions with the WattsBurning system evolved during the fifteen weeks of the study, we have studied this evolution by a series of Linear Regression analyses which generated 7 (one for each house and one for the total interactions) linear models that model the interaction taking into the account the week of the study. Out of the 7 generated models five 3 where significant (total, F2 and F1) with negative slopes, the rest of the models where not significant (although for 3 of them $\mathrm{p}<0.1$ ). The most relevant observation for this analysis is the confirmation 
that the total interaction decreased significantly during the period of the study $(\beta=-.18 .621, \mathrm{t}(17)=$ $-2.923, \mathrm{p}<0.012 ; \beta=-6.432, \mathrm{t}(17)=-2-759, \mathrm{p}<0.014 ; \beta=-5.384, \mathrm{t}(17)=-2.346, \mathrm{p}<0.003)$.

We also analysed the relationship of user interactions with energy consumption. The three households that on average didn't reduce their consumption are among the households that interacted less with the system (F4, F5 and F6). This finding is also consistent with our previous research. It is important to note that the system only logged direct interaction with the interface. It would be very hard to count the number of time the users looked or glanced at the tablet with the Energy Awareness mode on. Furthermore, users confirmed that a significant amount of interactions were made with the tablet in the Energy Awareness mode.

"It worked as an alert for me (...) it was easier to see from a distance (...) when we started to consume more it would get darker and catch fire" (F5, husband)

Table 3: Summary of the average of interactions with the system by day, in different periods of the study

\begin{tabular}{l|l|l|l} 
& \multicolumn{3}{|c}{ Average interactions by day } \\
Family & First 4 weeks & Rest of the study & Total \\
\hline F1 & 23.2 & 13.9 & 16.2 \\
F2 & 13.3 & 1.2 & 4.2 \\
F3 & 5.3 & 0.4 & 1.6 \\
F4 & 2.1 & 0.6 & 0.9 \\
F5 & 3.8 & 0.2 & 1.6 \\
F6 & 0.6 & 0 & 0.4
\end{tabular}




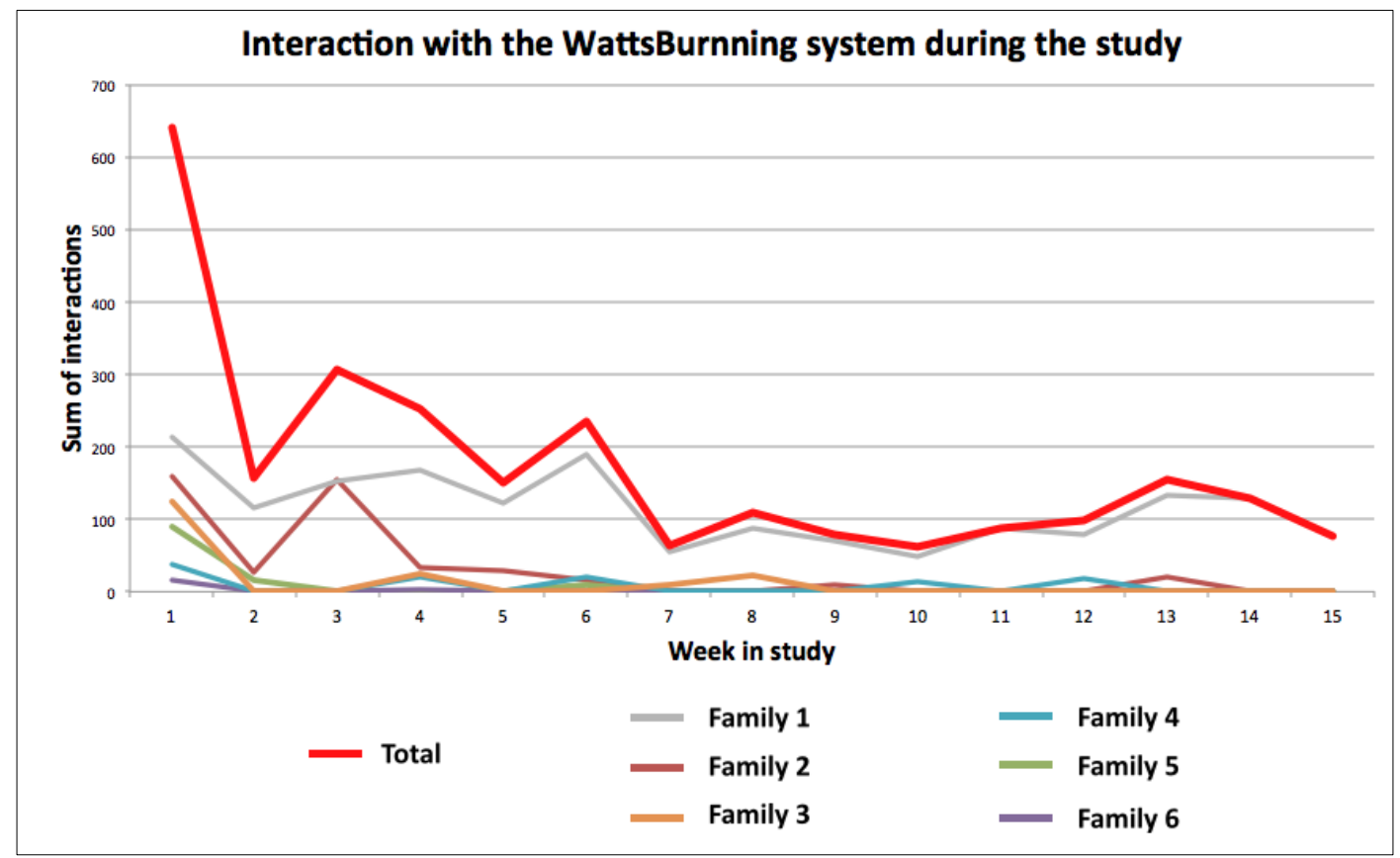

Figure 31: Interaction with the WattsBurning system during the fifteen weeks of the deployment.

\subsubsection{Discussion}

Our analysis confirmed that there was in fact a drop in consumption during the study, however considering all houses individually this change was not constant. Several analyses allowed us to state that during the WattsBurning deployment the consumption was different across all the households. Also considering the electricity consumption, it was verified that the electricity consumption was not significantly different at a particular day of the week, there is a general assumption that households consume more on the weekends however our tests did not confirm that statement. Although we verified that in fact certain houses reduced their electricity this observation was not constant across all the houses, yet, all families referred that the system helped them adjust their routing regarding energy consumption. We believe that this might be caused by the fact that the second part of the deployment occurred in the transition between Autumn/Winter, a period where traditionally there is more electricity consumption (mostly lighting).

The presence of eco-feedback increased the user's knowledge of the devices that they had at home. In fact, and besides that all the houses had different interaction patterns with the WattsBurning system, every participant learned something about a particular appliance, this indicates a rise in the awareness of energy consumption, despite the level of usage or the change in overall consumption. Portability allowed participants to place the device in a central place in the household, although it was not uncommon for it to be moved and used in other places 
After an initial period of a lot of interactions with the system, they started to decrease, this decrease was not equal in all houses, since certain houses kept using this system frequently for a longer period of time.

The loss of interest in eco-feedback after several weeks is an important factor leading to the relapse effect. Our novel eco-feedback system tried to overcome this issue by mapping energy consumption to elements of the natural landscape. From the interviews with users we can conclude that the mapping of the consumption with images of the local forest landscape was clear and well understood by participants. Which clearly improved the mapping from the SinaisFromFanal (section 4.4) which confused several participants.

Although users did not mention an emotional connection with the illustrated local forest landscape, the Energy Awareness mode, where the forest dries up as more energy is consumed, did work as an alert for when consumption was higher than normal. This usage pattern for abnormal consumption was found in other studies (e.g. one of the first eco-feedback studies published (Kohlenberg et al., 1976)), and it is widely referred as one way consumers interacted with ecofeedback devices. We argue that the visibility and glance ability of the Awareness Mode of the interface promoted this interaction type.

Some users found the forest on fire for a long time a disturbing factor that would motivate them to investigate which appliances were responsible for the higher level of consumption. Also, displaying the eco-feedback on a portable device allowed users to move the device through the household and explore the consumption of different appliances, yet families choose a central place in the household to place the system when it was not being used. In terms of usage the system had a lot of interactions during the first four to five weeks, after that period the number of interactions decreased. However, unlike our prior research the reduction was not linear and there were several peaks until the count finally settled at a low value. We believe this was a consequence of placement of the eco-feedback but also the presence of the Energy Awareness mode, which reminded people of the long-term consequences of their daily actions in the environment close to them.

The results in terms of user-interaction are an improvement over previous studies with classic quantitative forms of eco-feedback, since most families kept using the system after the four/fiveweek period although less frequently and with different patterns. This difference in how families use the system is in line with the "one size does not fit all" (He et al., 2010) argument for eco-feedback systems. If we relate the quantitative interaction data with the consumption information it is noticeable that the houses that saved more energy are the ones that used the system more which is inline with our observatins from the previous studies $(5.3 .2,5.3 .3)$. 


\subsubsection{Conclusions}

Our research with the WattsBurning energy monitor aimed at overcoming the problem of people relapsing to previous behaviour after several weeks of exposure to eco-feedback. Through an iterative design, testing and refinement process we improved our eco-feedback system with the introduction of a new metaphor that combines energy consumption levels with artistic representations of the local forest landscape.

Our goal was to verify whether this novel metaphor would continue to improve the amount of participant interaction with the eco-feedback system after four weeks of deployment. After initial prototyping that removed some of the ambiguity present in the mapping of consumption to natural elements, the WattsBurning system was successful in improving the levels of user attention and usage over time. Most families kept using the system even after four to five weeks, although to a lesser extent. The placement of the eco-feedback device and the presence of an energy awareness mode showing the landscape changing was an important motivator to retain user attention and awareness over time. These findings provide a good motivation to explore new forms of ecofeedback that go beyond traditional quantitative information. The households in our study did manage to reduce their consumption, but they showed very different consumption and eco-feedback usage patterns. Overall, households that used the system to a greater extent saved more energy. We believe our attempts with non-conventional forms of eco-feedback and, in particular, when exploring emotional and aesthetic aspects is a promising path to explore further. 
Research Studies 


\subsection{Social enabled tangible eco-feedback: WattsBurning in my mailbox}

This chapter describes an attempt at using the social influence as motivation to reduce electricity consumption (section 3.5.2 presents a series of other studies that looked at the same dimension). With the WattsBurning in my mailbox study we employed the WattsBurning ecofeedback strategy as an analogue public energy consumption visualization for displaying in a public areas of an apartment building, in particular, the physical mailbox. This chapter firstly presents our motivation for the study, next we present how the public display was designed and deployed, and finally we conclude with the results of a study with the WattsBurning in my mailbox public display.

\subsubsection{Motivation}

As a further extension of the WattsBurning deployment (previous section) we experimented with a tangible version of the eco-feedback visualization aimed at studying further the mapping of households' consumption into the local forest. Motivated by the public displays work in ecofeedback (Foster et al., 2010; Odom et al., 2008) it was decided to investigate this technique as the next step in our research. Additionally, with the WattsBurning in my mailbox study we also aimed at taking advantage of the inherent pedagogical qualities of tangible interaction (Antle et al., 2009; Bakker et al., 2011; Horn et al., 2012), especially in the younger demographics in the households. This experiment utilized the same households sample gathered for the WattsBurning system (section 4.5.5). At the time of this study the families were still using the digital WattsBurning system described above, while we added the public display component to it. Both studies were finalized at the same time.

\subsubsection{Public display design}

Since this was our first attempt at a public display for electricity consumption it was decided that apart from seeking guidance in literature, it would be important to gather insights from researchers with experience in the field. With this goal in mind, a workshop was organized, where researchers, designers and artists came together to think about innovative ways of providing public eco-feedback beyond the traditional informative $\mathrm{kWh}$ or $\mathrm{CO}_{2}$ emission displays. Several of the workshop members had previous experience with public communal eco-feedback, in particular the TidyStreet initiative, which was part of the Change project (Bird and Rogers, 2010). 
The brainstorming session revolved around the combination of a public (communal) display and using forms of an artistic display connecting the energy consumption to elements of the environmental heritage of the local place. Several ideas emerged from the workshop, most of which looked at tangible ways of providing and sharing the eco-feedback from the households. The research team agreed that art and playfulness could play a major role in increasing the emotional connection between people, their energy consumption and the long-term effect on the environment, (motivated by, among others, the work of (Gustafsson and Gyllenswärd, 2005; Jönsson et al., 2010)). Ideas ranged from using a portrait of the family with different moods to map different consumption ranges, to a local newspaper reporting on the consumption of the apartment building as a ranking. A public display was then chosen as the appropriate option for households to share their consumption, since we wanted to experiment with social challenges and competition as motivator for energy conservation (more on social eco-feedback in section 3.5.2). This way, the team started looking at public spaces that could be used to share the consumption data gathered by eco-feedback.

Considering that the system would be deployed in apartment buildings the physical mailboxes provided an interesting place, which was both accessible and visible to the larger community, from which to share the consumption information. The mailboxes could act as placeholders for tangible displays of energy information that would at the same time be shared with their neighbours. In order to leverage the connection between the in-house system that was already installed and the newly shared eco-feedback, the research team brainstormed different ways to bring the artistic visualization of the consumption outside. It was decided that the mailbox would depict the consumption through a set of artefacts, in our case magnets, that had to be placed by the users according to their consumption.

\subsubsection{Mailboxes Magnets}

The visualization presented on the mailboxes was part of the forest landscape that was used in the tablet eco-feedback application.

The system provides an opportunity for family members to share their energy consumption information already accessed through the tablet application at home, using a shared physical space outside of their apartments. Both eco-feedback visualizations mapped household consumption to the natural endemic forest. See Sections 4.4.1 and 4.5.1 for the design rationale behind including the local nature in the electricity consumption eco-feedback mechanism.

The hardware used to implement the outdoor visualization was based on a set of magnets that participants placed on their mailboxes according to their daily consumption. Each magnet represents 
the consumption of a specific day of the week, referring to part of the full illustration of the local forest at three different health stages: "sick", "normal" and "healthy". The landscape depicted here is a horizontal portion of the landscape present in the tablet system with which users were already familiar (see 5.5.3). In total each family was given 21 magnets, three for each day of the week with the three different health levels. In addition, there was also a banner placed above the magnets with the caption for each day (see Figure 32). Since the mailboxes were made of aluminium (the magnets did not stick to it) a small-magnetized rubber band was placed in the mailbox through hooks so that the magnets would "stick" to it. Figure 32 shows the banner and the three different landscapes that created the set of magnets for the three different consumption levels. Figure 33 shows how the magnets were placed on the mailboxes.
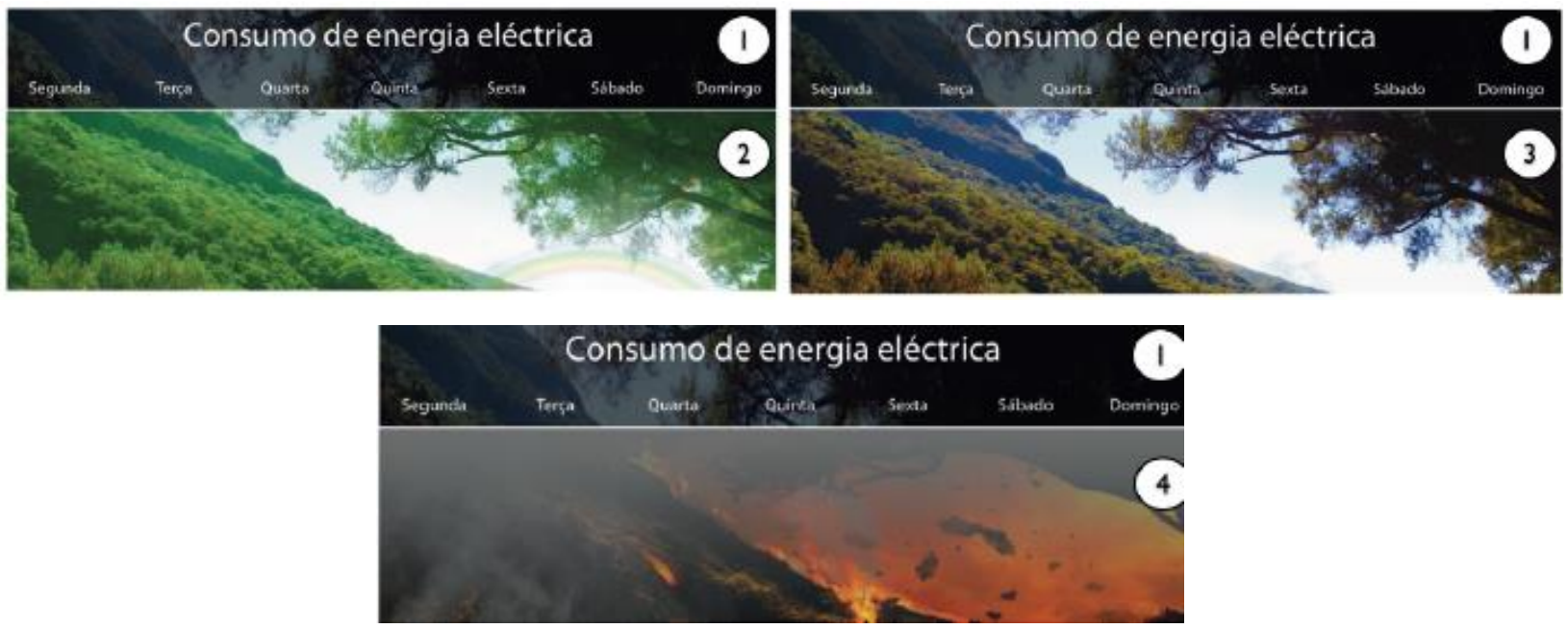

Figure 32: The 3 different stages of the forest represented by the magnets (banner is on top). 1. Banner with the week days. 2. Forest with low consumption. 3. Forest with medium consumption. 4. Forest with high consumption.

\subsubsection{Evaluation}

To evaluate how sharing energy through the Wattsburning in my mailbox system impacted the perception and energy consumption we selected 4 families for a period of one week and asked them to place magnets on their mailbox every day. The sample families for this study were a sub-sample from the families selected for the bigger deployment of the tablet-based eco-feedback system (section 4.5.5). Therefore, the families were already used to receiving information about their energy consumption, and they were aware of how their routines impacted the presentation of the forest, e.g. High Consumption: the forest is burning, Low Consumption: the forest is lush. All the families were couples with children; in 3 of the families, the children were younger than 12 years old. The families lived in two apartment blocks from the same building complex in an urban area in the city of Funchal, 
on Madeira Island. Figure 34 displays how the selected mailboxes were positioned at the apartment complex. The mailboxes were placed at the entrance of each apartment block and visible to passersby and neighbours. Users were recruited for this study by phone and then scheduled for an interview later to explain how the mailbox system should be used.
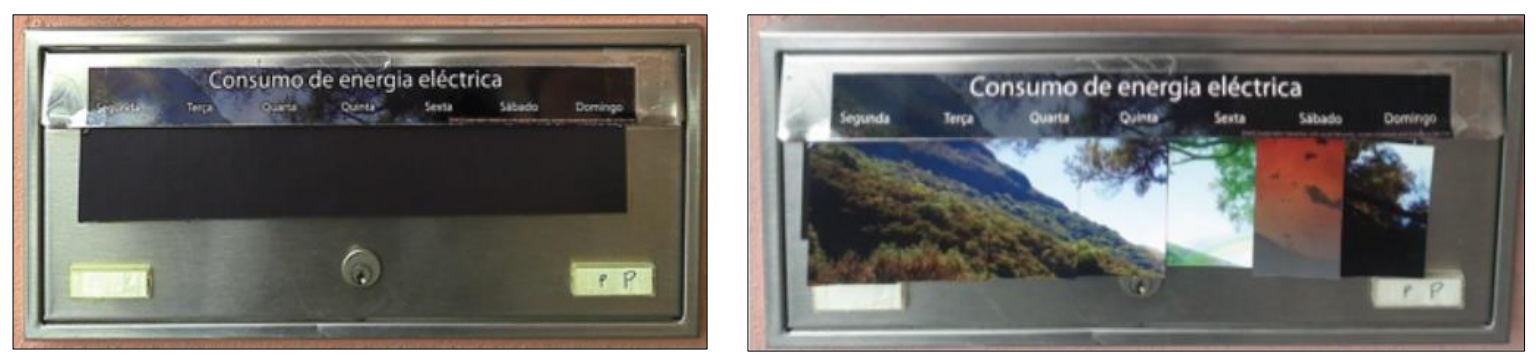

Figure 33: Mailbox of one participant at the beginning (left) and at the end of the study (right).

\subsubsection{Methodology}

The mailbox study lasted one week, which is understood from previous research (Filipe Quintal, 2012; Nunes et al., 2011; Pereira et al., 2013), as enough to span the family routines that impact energy consumption, and the mailbox interface was designed with that timeframe in mind. Each morning at 7:00 AM the participants received a text message with information about their consumption of the previous day. The text message was sent in the form of this template:

\section{Good morning Mr/Mrs "Participant name" your consumption yesterday was abovelequal/below your average for that day of the week, you should proceed to place the magnet assigned for high/average/low consumption.}

Then we expected family members to go and place the magnet in the corresponding position on the mailbox outside the apartment building. At the end of the afternoon a member of the research team passed by the building to check if the participants had placed the magnets correctly (participants were not aware of this verification). In the following week, we visited the families and conducted a short interview. The goal of the interview was to qualitatively verify if the metaphor used to represent the consumption was well-understood, how the family performed the task, and how families felt about having their consumption displayed outside their houses. Appendix 9.4 presents the protocol following during the WattsBurning in my mailbox study. Figure 33 displays how the magnets were placed in one particular household. 


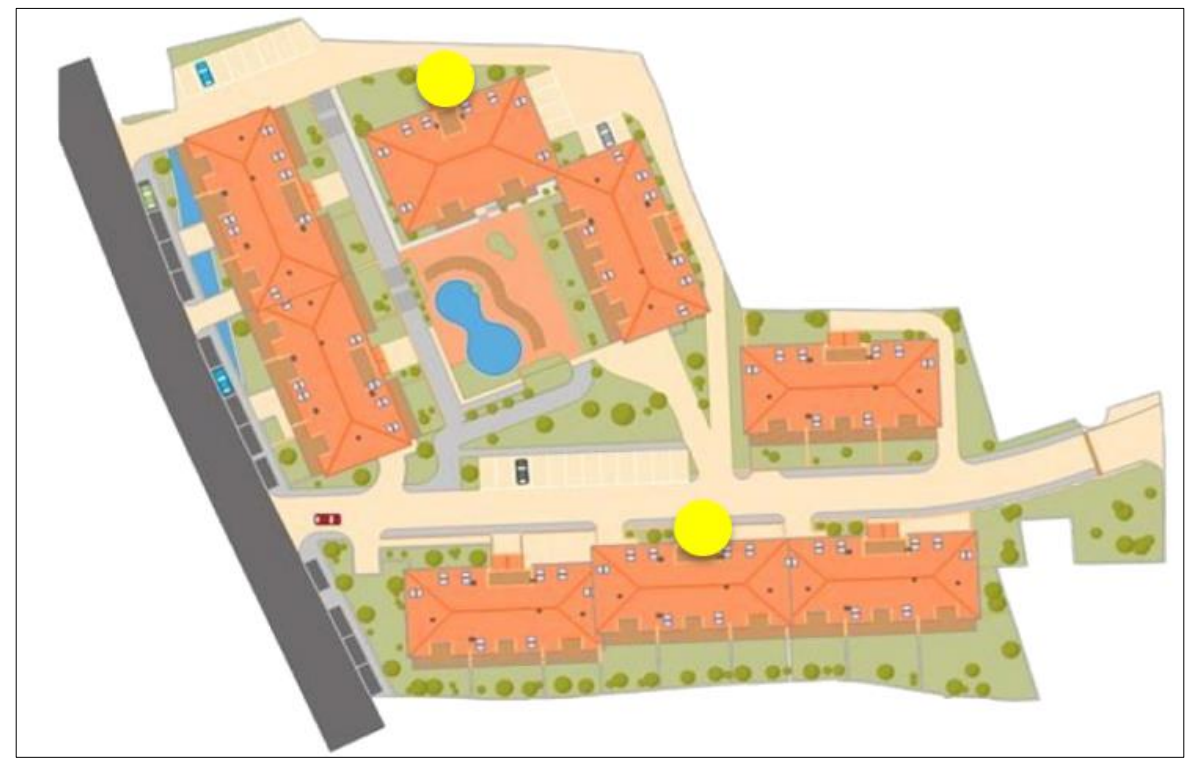

Figure 34: Image of the apartment complex used in the WattsBurning on my mailbox study. The yellow dots mark the positioning of the mailboxes in the selected buildings.

\subsubsection{Qualitative analysis}

From our afternoon observations we confirmed that only one of the families skipped the magnet placement on one day. However, they placed two magnets the following day. Apart from that case all the families performed the task on time and correctly (the placed magnet was correct according to the consumption).

All families found the task of getting the message and placing the magnet simple and reported that it did not interfere with their normal daily routines. They also found the magnets easy to understand. None of the families felt discomfort or concern about having their consumption displayed outside their apartments and visible to neighbours or passing people:

"It was indifferent... I was not at all concerned" (F1, wife)

Although the consumption was only broadly exposed through the colour of the landscape "slice", when asked if they would feel the same way if actual consumption data in the form of numbers was displayed, instead of the forest representation, families again reported minor privacy concerns, but there was no strong opposition to it:

".. Of course there could be some privacy concerns... but I don't see any negative aspect with that" (F3, husband). 
It is important to note that we asked families about a hypothetical scenario, their reaction might have been different from their a priori answer once they could actually see the numeric information outside their house.

Particularly interesting reactions to the system came from the children. In one of the apartment blocks the children from the two families that participated in the study were friends, and in both cases they quickly became responsible for performing the task:

"He went there before going to school, he asked me for the consumption, and he went there ... I have got the stack with the magnets, he chose it before going to school" (F2, husband).

"She went there before leaving to school. She got the magnet from the stack and we stopped in the mailbox before leaving". (F1, wife).

These statements suggest that competition was created between the children and that this unspoken competition soon became apparent to the adults in the families:

"It seemed that they were competing with each other... every day in the morning she asked who spend more us or them... at the end she told me Who won?. It was more a competition between them, she was the one reminding me to check the message" (F1, wife)

"Normally we don't spend without being necessary, but there was a day with higher consumption. He came to asked me why is that? I had to explain him that his mother was cleaning and so on". (F2, husband).

In the other two families (from a different block), the task was performed by the father, (House 3 ) and the mother (House 4). From the interviews it was noticeable that while still not being too intrusive, the task of getting the message and placing the magnet was seen more as a chore by these two families. In one of them the mother stayed at home and felt like she did not need to ask anyone else to do the "task", and in the other family the father was truly interested in and controlling over the energy consumption, and saw this task as another of his responsibilities as the member of the family who controlled consumption at home. The positioning of the mailboxes meant that it was almost impossible for neighbours to ignore each other's consumption. This was confirmed during the interviews, two of them were actually curious to check:

"I got surprised in the end, our values were close (comparing with the neighbour), but of course since we are comparing savings the actual consumption values could be different)" (F3, husband) 


\subsubsection{Discussion and Conclusions}

From our short study we argue that tangible interaction and public sharing of data seems a promising approach to bring awareness to resource consumption. In this study we continued our research in innovative ways to provide eco-feedback that leveraged an emotional connection of people with their consumption and the long-term effects while at the same time making use of some playful strategies to raise awareness.

WattsBurning on my mailbox' provides a novel and playful strategy for families to display and share their energy consumption. Our results show that the task of placing magnets reflecting daily energy consumption outside of the houses was not considered too difficult or a burden by the families. However, since this observations are based on a one week study, different reactions could arise if the study lasted longer. When given the opportunity, children become the gatekeepers for updating the visualization at the mailbox, and we observed that the magnets function as probes and awareness aids for the adults about their consumption. We believe that this can provide opportunities for children-oriented eco-feedback systems that are more playful and stimulate communication about routines and energy consumption patterns inside and across families. We also position our work among the body of work that considers tangible interaction as an efficient approach for teaching (Antle et al., 2009; Bakker et al., 2011; Horn et al., 2012), in this case from parents to their children.

Our results with the WattsBurning in my mailbox study verified literature claims that public displays can promote competition among individuals (section 3.5.2 of the related work). This particular aspect was mostly visible among the youngsters in the apartment building. We believe that our study attracted children on the grounds of the abstract and simple visualization and by the playfulness of the task itself.

Like we mentioned before, participants did not show any particular concern about having their household consumption publicly displayed outside. We argue that this result contributes to the body of work on social eco-feedback, by confirming that consumers are willing to share their consumption. 
Research Studies 


\subsection{Connecting electricity consumption with electricity production, the What-a-Watt system}

The work described in this chapter was motivated by the series of studies in which we attempted to connect individuals with their consumption (section 3.5.3 and 3.5.4). However, in this study we planned to explore this connection further. This way, instead of using metaphors or abstract representation of individuals' impact in the environment (see section 4.4.3 and 4.5.3). Individuals are presented with the raw resources used to produce the electricity consumed in their household. Moreover, we were informed by previous work that aimed at presenting consumers with the actual source of electricity (see section 3.5.4 of the literature review). This subset of eco-feedback studies includes information about electricity generation to consumers, which possess photovoltaic or windmill installations. With the What-a-Watt study we expanded that approach by including whole island disaggregated electricity production information in the eco-feedback, the next sections explain in detail our motivation, design and evaluation of the What-a-Watt eco-feedback system.

\subsubsection{Motivation}

Through the work presented in this chapter we proposed to advance the state of the art with a novel approach to eco-feedback that connects production and consumption information. Unlike the previous approaches with micro-generation (Pierce and Paulos, 2012b), we extend the concept to a whole-island isolated network with a considerable (30-40\%) penetration of renewables. Our deployment occurred in a controlled environment on a medium-sized island with a closed grid of approximately 270000 people. This unique setup works as a "large-scale micro-generation" and we provide information about the source of electricity (thermal, wind, solar or hydro) in real time. The local grid is composed of three thermal stations that burn fuel/diesel and waste/biodegradable material; seven wind parks in the mountains; ten hydro stations and approximately 700 small private domestically owned photovoltaic installations that feed energy into the grid.

In summary, the goal of this work was to verify if the high levels of awareness and concern shown by micro-producers can be brought to regular consumers. With this controlled experiment in a closed network we envisage future scenarios in larger networks where consumers could be presented with new service models for electricity that promote sustainability. 


\subsubsection{The What-a-Watt eco-feedback system}

To explore the connection between production and consumption we designed the What-aWatt eco-feedback system. In this section we describe the design and implementation of the What$a$-Watt eco-feedback system. The sensing platform used for the What-a-Watt application was based on the second version of our Non-Intrusive Load Monitoring framework (section 4.2.5).

\subsubsection{System design}

In this section we describe the main design decisions during the implementation of the What$a$-Watt system, ranging from the hardware to the screens displayed in the interface.

\subsubsection{Eco-feedback}

The feedback was given using a 7" android tablet. The system was developed using native development SDK. The Android application is composed of a set of tabs that represent the production information and summary of the consumption on a daily, weekly and monthly basis.

The production view is the default mode of the application and the system reverts to this visualization when no interaction happens for a pre-defined period of time. The application never goes to idle mode as is common with several android applications. The design of the views that represent consumption over the day, week and month are an evolution from the ones presented at the WattsBurning which had already been tested with users in a real world scenario for 17 weeks (Quintal et al., 2013)(section 4.5).

\subsubsection{Consumption feedback}

The summary view contains two charts representing the consumption of the current day, week and month, and a comparison between homologous periods. Also on this tab the system presents the user with a "tip of the day" with general sustainable recommendations (see Figure 37 left).

The "Day", "Week" and "Month" views contain a chart with the consumption during the selected time period. There is also information about the total aggregated consumption, the peak consumption and how it compares with the baseline for that period. 

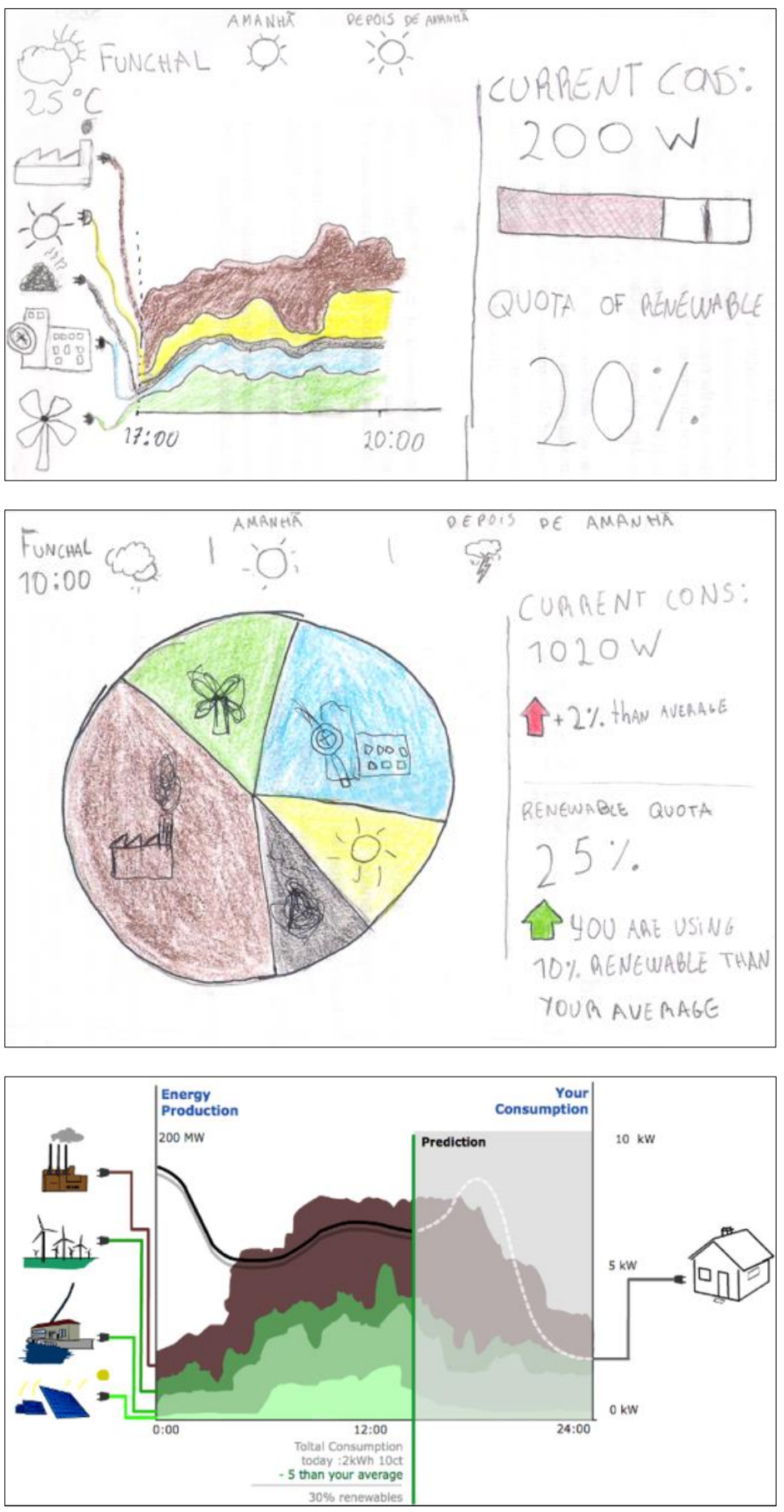

Figure 35: Different interface prototypes sketches developed during the design of the production view for the What-a-Watt system.

\subsubsection{Production feedback}

The biggest challenge when designing the interface for the What-a-Watt system was to depict the data about the electricity production. Our design process started with meeting with experts from 
the local energy and environmental agency and the local electricity provider since both these entities have a deep knowledge of the local grid. From these meetings we understood the different techniques used in managing production in the island and how variables such as weather, time of the day and consumption demand, influences where the electricity comes from. This then allowed us to understand how the production data was grouped, how frequently the production values were updated and the expected quotas in different situations (for example day vs. night or rainy day vs. windy day). Those insights influenced the design of the eco feedback visualization. Several prototypes of possible visualizations were generated. The ideas revolved around representations that allowed the production information to easily visible and distinguishable. A small qualitative evaluation was performed with approximately 10 individuals (colleagues from the lab and the university) in which we tested figurative prototypes where the electricity sources were represented visually with graphics representing the origin of the produced energy (water, wind, sun and waste). We also considered more abstract prototypes such as pies and bar charts representing the quotas of renewables. See Figure 35 for an example of the generated visualizations.

The visualizations were tested with users through wizard-of-Oz and think aloud evaluation techniques. The final selected prototype was based on the users' preferences, ease of use and understanding. Subsequently we proceeded in creating a design language consistent throughout the application.

The selected visualization shows a "cumulative" chart representing all the sources of energy used during the day, their quotas relative to each other, and a prediction of which sources will be available for the rest of the day (Figure 36). For example, at 18:00 the chart displays the quotas of the different sources so far, and their availability for the rest of the day. Each source of electricity has a colour associated with it: yellow for solar, light blue for hydro, light green for wind, and dark grey for thermal. This choice of representing the renewables with light colours and the thermal source with a darker tone, allows for the user to get an immediate overall perception of the amount of renewable energy present at grid at each moment, by effortlessly glancing at the display (Figure 36). This view also presents the total percentage of renewables used during the day, and the average of each source also during a particular day.

Additionally, the production view presents a high-resolution chart of the daily consumption, in which the data is grouped in five minutes' intervals. This chart is superimposed with another dotted line chart displaying the average consumption for that day of the week (Figure 36). This allows users to confront their real time consumption with the different sources available at the time, and with their average. We were also expecting that the high-resolution chart would allow consumers 
to identify single appliances, or everyday activities such as cooking lunching or playing a videogame console thus enabling consumers to extract more information from their consumption data. This observation was reported from literature (e.g. (Broms et al., 2010)), and we believe it is an inexpensive way to provide disaggregated consumption information (compared with installing sensors in each appliance). The dotted vertical line on the production chart represents the current time of the day, the production values in the right represent the predicted production based on the model used by the electrical company. Similarly, the grey vertical line represents the current time of the day in the bottom chart.

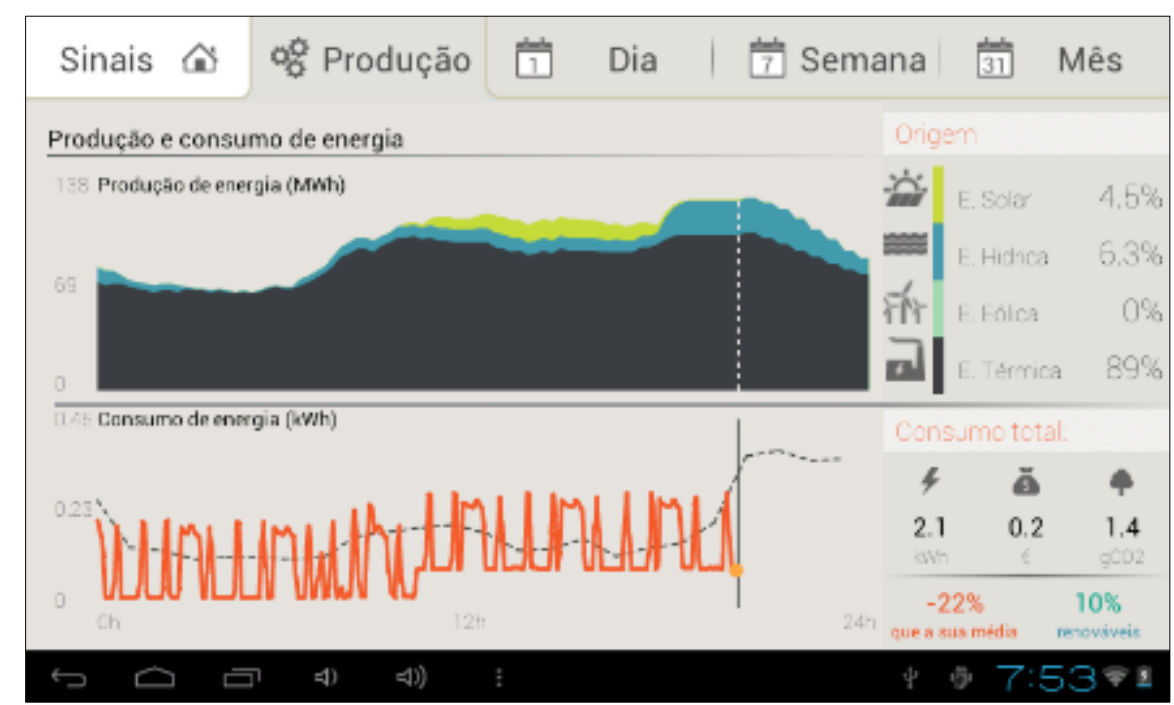

Figure 36: Interface screen displaying the island electricity production and the household consumption. In this particular moment there was $10 \%$ of renewable energy present in the grid, and the household was consuming less $22 \%$ than its average.

\subsubsection{Production data}

The local electricity production quotas are obtained directly from the local energy provider which is responsible for the operation of the electric grid, as well as the producer of the majority of the electricity consumed locally. Every fifteen minutes a web-service is updated with the disaggregated quotas for the electricity produced. A script queries the web-service and inserts that data into our database, the production data is then made available through a series of services providing real time, historical and comparisons production data. Additionally, the local supplier also provides the outcome of a model used to predict the regional production for the next twelve hours, this model is used in their internal operation, and we handle this data in a similar manner to the production. 


\subsubsection{Collected data}

The sensing framework computes consumption at 50 samples per second and stores the average consumption every other minute in the underlying database. These consumption values are used to compute the average per hour used in the analysis and on the different charts of the application. Additionally, if necessary the system accesses higher resolution data ( 2 consumption points per minute). The system also records all the transitions between the different interface screens, which provide a good metric to understand how the users interact with the system. An interaction with the system was considered every time the user selected one of the different tabs of the interface, or touched one of the buttons.

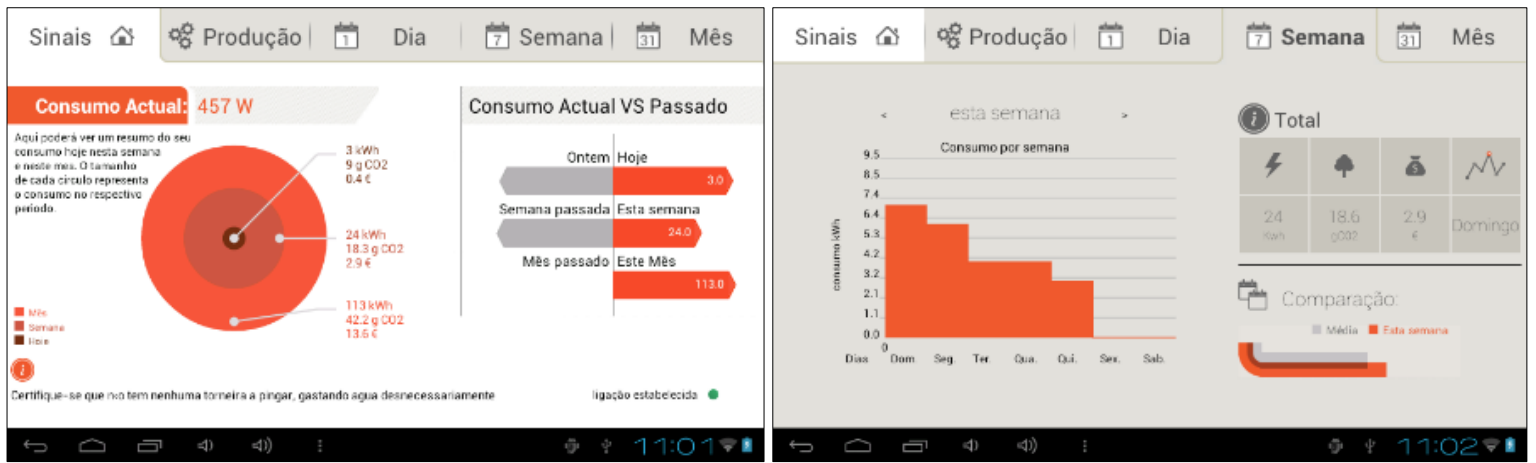

Figure 37: Left: Home view of the What-a-Watt system displaying averages and comparisons of consumed and produced energy between time periods. Right: Interface screen displaying the consumption throughout the week.

\subsubsection{Evaluation}

To evaluate the What-a-What system we selected 9 families from a nearby apartment building corresponding to the average consumption households in the island's main city with more than 50 000 residential consumers. The research team surveyed several buildings, with different demographic and consumption profiles, looking for a building where the residents matched the medium salary income and energy consumption profile. Members from the selected families had different occupations including one unemployed, two artists, two businessmen, two professors, one lawyer, two waiters, two bank clerks, one human resources manager one appraiser, one economist, one marketing manager and one agronomist. Five families were selected to use the What-a-Watt application (total of 12 individuals) and four others acted as a control group where the consumption information was measured but no feedback given. The sub sample of five families that used the What-a-Watt system was composed by one middle aged man, a couple without children, another 
with one baby, one with 2 children and a family of two, composed by a mother and her daughter. Adult's ages ranged from 25 to $45(\mathrm{M}=37)$.

This study lasted for 34 consecutive weeks ( 32 weeks of study +2 weeks of baseline), we argue that a long-term study is required in order to fully understand if the awareness shown by microproducers can be passed to regular consumers. The production of energy is highly conditioned by external phenomena like the weather (without rain there is a small quota of hydric energy in the grid) and daylight (solar energy varies with the number of sunlight hours). Our long deployment guarantees that consumers are exposed to different conditions helping them to assimilate the reasons behind the different quotas presented by the What-a-Watt.

\subsubsection{Method}

We asked all the families to complete a questionnaire with general information regarding electricity consumption and demographics at the time of recruitment.

The sensing framework was installed and baseline data (without feedback) was collected for a period two weeks. After this period, the five participating families were given a tablet with the What-a-Watt system installed and instructions on how to use the system. With this procedure we guaranteed that all the houses had two weeks of baseline consumption allowing for the individual analysis of each household's data. One week after the system was installed, the research team visited families to clarify any problems they might have had with the system, and to guarantee that everything was working correctly

Nine weeks into the deployment we visited the families and asked them to complete a small questionnaire regarding the use of the eco-feedback system, including the perception of potential savings of electricity (Appendix 9.6). The questionnaire was analysed in conjunction with the interactions and consumption data. The outcome of this analysis was then used to adapt a structured interview for each family (Appendix 9.7 presents the base protocol that was then adapted based on the outcome of the questionnaires). Interviews with families lasted between 20 and 45 minutes, and were recorded and transcribed to text.

In this chapter we present the findings up until the end of March 2014, corresponding to 32 consecutive weeks (approximately 8 months) of usage of the eco-feedback system (plus two weeks of baseline data). In this section the participating families are referred to as F1, F2, F3, F4 and F5. 


\subsubsection{Quantitative assessment}

The quantitative data gathered in this study was grouped by week before the analysis. Weekly consumption provides a good metric to group all energy related routines in a household. The only exception is the baseline consumption since we had two weeks of data and therefore used daily consumption to identify trends.

\subsubsection{Interactions with the What-a-Watt system}

On average there were nine interactions per week for each household during the 32 weeks of deployment $(\mathrm{M}=9, \mathrm{SD}=30)$. However, the interactions with the system reached a peak in the first four weeks of the study, averaging 52 interactions per household in that period.

This is consistent with previous findings reported in literature (e.g. (Quintal et al., 2013), sections 4.5), which estimate the novelty period for eco-feedback to be, in average, four weeks. After that the interactions with the system decreased to an average of three interactions per week per household. The actual interaction values differed between all households in the study, however this difference was not enough to return significant differences when this interaction data was submitted to and ANOVA statistical test, furthermore an Tukey HSD did not returned any significant pair (for $\mathrm{p}<0.05)$.

The most accessed view was the "Home" view of the system, followed by the "Production view" (this pattern was consistent in all the installations). There were no observed patterns regarding the accesses of the feedback system by time of day or day of the week. Table 4 shows the interaction values for all houses in different periods of the deployment (the count of interaction values were rounded to the unit).

The evolution of the frequency of interactions with the What-a-Watt system was also studied fitting linear models to the data and comparing the slope of the fitted line. Six linear regression analyses were ran, one for each house and one for the total interactions, all of the regressions were significant with negative slopes in all houses $(\beta=-1.0598, \mathrm{t}(31)=-2.430, \mathrm{p}<0.012 ; \beta=-1.2874$, $\mathrm{t}(31)=-2.265, \mathrm{p}<0.030 ; \beta=-0.7279, \mathrm{t}(31)=-3.175, \mathrm{p}<0.003 ; \beta=-1.9362, \mathrm{t}(31)=-2.136, \mathrm{p}<$ $0.04 ; \beta=-1.0598, \mathrm{t}(31)=-2.430, \mathrm{p}<0.021 ; \beta=-1.212, \mathrm{t}(31)=-3.453, \mathrm{p}<0.001)$.

Using the slope of the linear fit, the average decrease was also calculated, in average families used the system $82 \%$ less in the last week of the study ( $\mathrm{SD}=10 \%)$. 
Table 4: Count of interactions with the What-a-Watt system during different periods of the study

\begin{tabular}{l|l|l|l}
\multicolumn{4}{|l|}{ Average Weekly interactions with the What-a-Watt system } \\
\hline Family & First 4 weeks & Rest of the study & Total \\
\hline 1 & 50 & 4 & 9 \\
\hline 2 & 88 & 4 & 14 \\
\hline 3 & 22 & 5 & 7 \\
\hline 4 & 53 & 3 & 9 \\
\hline 5 & 50 & 2 & 8
\end{tabular}

\subsubsection{Electricity consumption}

Three different approaches are combined to understand the variations in consumption during the period of the study. Firstly, the control group is used to compare the consumption of households using the What-a-Watt with houses without any feedback on electricity consumption. Second, the fifteen days of recorded baseline consumption is used to compare the consumption before and after our intervention. Lastly the overall consumption trend is also analysed. One family (F1) had to leave the apartment in the 14th week of the study, the results presented below take that fact into account by removing family 1 from all the averages after the 14th week.

To compare difference between groups during the study, the consumption was averaged by week, houses in the study had an average of $47579 \mathrm{Wh}$ by week $(\mathrm{M}=47579, \mathrm{SD}=7970)$, and house in the control group averaged $65471 \mathrm{Wh}(\mathrm{M}=65471, \mathrm{SD}=12924)$ by week. These two groups were subject to an independent $t$-test, which returned significant differences among the houses. A paired samples t-test was used to compare the consumption in the study between the baseline and study periods (only in houses using the What-a-Watt system). This test did not reveal any significant difference (for $\mathrm{p}<0.05$ ), however it is important to note that for this test the sample was heavily in unbalanced, with 2 weeks for the first period and 33 for the second. This way other approaches were used to analyse how the consumption evolved during the study. 
We used the slope of the linear regression fit to the average weekly consumption to study the evolution of the electricity consumption in period of the study. Three regressions were ran, and the percentage difference was calculated using the slope of the linear fit. This way during the 32 weeks deployment, the group of all houses in the building increased their energy consumption by $14 \%$, Figure 38 red line, however this regression was not significant with $\mathrm{p}=0.08$. Families using the What-a-Watt system decreased their consumption by $18 \%$ (Figure 38 orange) and houses without any feedback increased their consumption by $41 \%$ (Figure 38 green) $(\beta=-1.212, t(31)=-3.453, p<$ $0.001 ; \beta=-2686, t(31)=-2.628, p<0.022)$.

Figure 38 displays how the electricity consumption evolved in the three groups (houses that used the What-a-Watt system, control houses, and both), we have chosen to display average consumption by week in the chart since it accounts for F1 leaving the study.

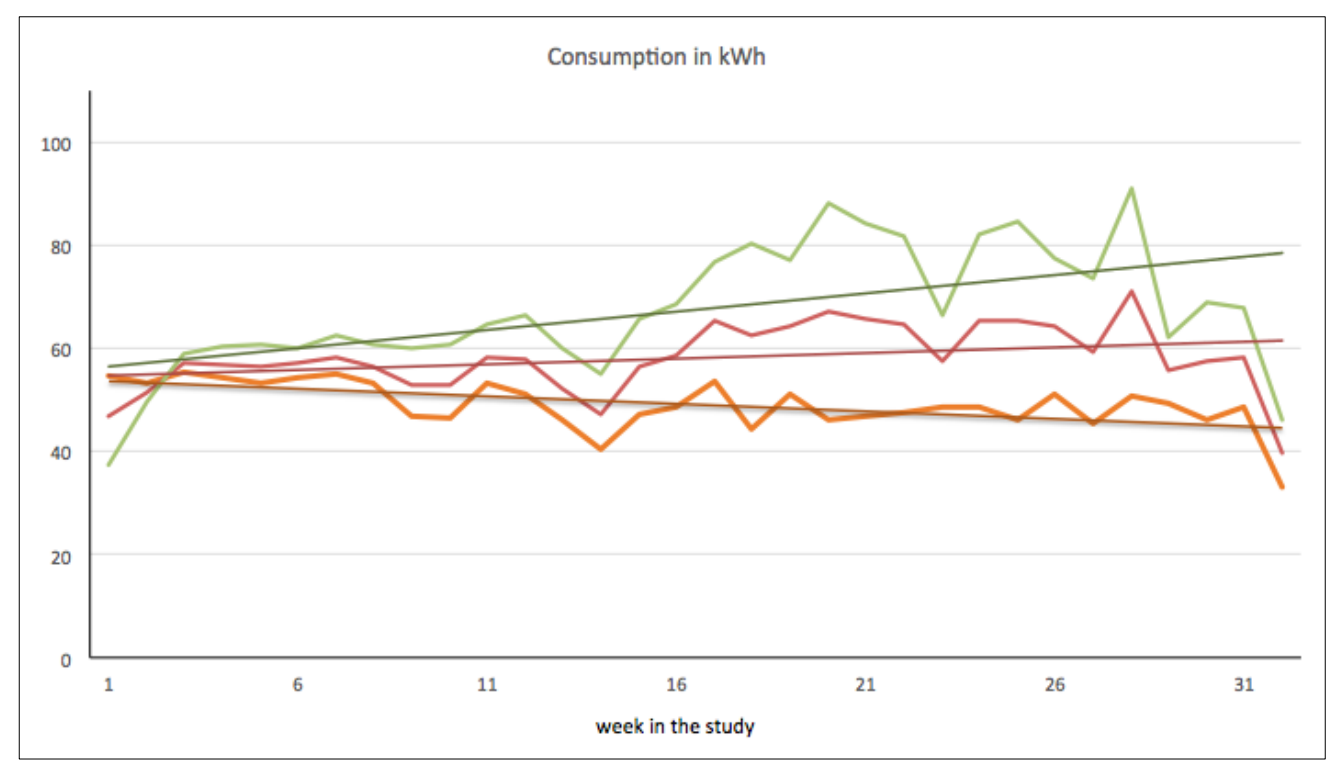

Figure 38: Time series with the average weekly consumption throughout the study in the three groups: Orange line - Study group; Green Line - Control group; Red line - Whole building.

\subsubsection{Power events and consumption routines}

To understand family routines regarding electricity consumption we investigated the power events since this metric is highly related to the usage of appliances (power events are described in section 4.2.3). Power Events were organized in 8 categories based in their consumption:
1. $[0 \mathrm{~W}-50 \mathrm{~W}]$;
2. $[350 \mathrm{~W}-450 \mathrm{~W}]$; 

3. $[50 \mathrm{~W}-150 \mathrm{~W}]$;
4. $[450 \mathrm{~W}-550 \mathrm{~W}]$
5. $[150 \mathrm{~W}-250 \mathrm{~W}]$;
6. $[>550 \mathrm{~W}]$
7. $[250 \mathrm{~W}-350 \mathrm{~W}]$;
8. All events.

Through this analysis the categories listed above will be referred as category $1,2,3,4,5,6,7$ and 8.

Considering the amount of events happening in each household, category 2 events occurred more often in all households. Category 1 was the second most "present" in 4 households (in family 5 category 1 was the 3 rd and category 3 was the second). In fact, the first 3 categories (events with consumption smaller than 250 watts) account for an average of $84 \%$ of all events in the household $(\mathrm{N}=649732, \mathrm{SD}=5 \%)$.

Looking at how the power events happened during the day, we divided the day in different time periods, which represent the normal day-to-day routine of a family: breakfast, work, lunch, dinner, night and dawn. In average $67 \%(\mathrm{~N}=649732, \mathrm{SD}=10 \%)$ of the events happened in the period that individuals were at home (breakfast, lunch, dinner, night and dawn), 72\% (N=649732, SD=14\%) happened in the night/dawn period. Furthermore, the night period, six hours from $7 \mathrm{pm}$ to 1 am holds a big cluster of events that account for $66 \%(\mathrm{~N}=649732, \mathrm{SD}=15 \%)$. The small standard deviation from the previous averages hint that different houses shared consumption routines. To further investigate this observation, we ran 160 correlations between every category of power events and every household. For this analysis every event in household was counted by hour of the day, resulting in 24 data points for each house and for each event category. Eighty five correlation were significant $(p<0.05)$, from those forty four had a value bigger than 0,6 . The significant correlations did not follow any particular pattern which lead to conclude that even though households share high level routines such as lunch or dinner, the actual individual appliance usage is very different among households.

\subsection{Qualitative assessment}

In this section we analyse the different interviews with family members during and after using the What-a-Watt system. After transcribing the interviews, we organized user's answers into topics that grouped their impressions about the eco-feedback device. Our analysis disclosed the following 
three topics: Feedback usage, problems and improvements, Awareness of the electricity consumption and Electricity production.

\section{Feedback usage, problems and improvements}

The eco-feedback was well understood and received and none of the families had any problems using the system. The eco-feedback system clearly increased consumers' awareness of their electricity usage, this observation was expected (from literature) and it was consistent within all the households. Families referred that the system alerted them to certain behaviours, and also allowed them to follow their energy consumption closely as the mother of F2 explains:

"I used to come here to check the real time consumption, also the weekly, monthly and daily consumption... also every time we turned an appliance $O N$ we checked its consumption... here in the real time values, we can get an idea of which one consumes more or less". (F2, wife)

Families referred that the system could be improved by adding features such as a comparison tool with other similar families, like it was pointed out by F4:

"It would make sense to have a way to compare with other families, I would use it the same way I use the comparison with the monthly or weekly average". (F4)

It was also mentioned by F2 that it would be interesting to get consumption information by appliance or by room:

"It would be interesting to walk with the device in the house and check in which room there is more consumption, check which devices are spending more at each time, and for example turning on a light bulb and knowing how much it is consuming" (F2, wife).

However, families also referred that the high-resolution chart in the "production view" of the system allowed them to trace individual appliances as the daughter in F2 explains:

"there is a peak here during lunch, because today we used the microwave, I am sure it was the microwave, we detect it as soon as it was turned on" (F2, daughter).

\section{Awareness of electricity consumption}

Families stated that the system alerted them to certain behaviors, and also allowed them to follow their energy consumption closely. All the families mentioned that they were already concerned about their electricity consumption and sustainability. Actions like changing to low-power 
light bulbs, buying more efficient appliances or opening the curtains to allow sunlight to get through, were already adopted. However, the What-a-Watt system allowed them another level of control over their actions by matching their perceptions with actual consumption data. F5 mentioned that the system worked as a reminder, alerting them to perform or do something that they would normally do but sometimes forgot. Another consistent observation across all households was how easily families were able to trace abnormal consumption routines (for example a peak in the daily consumption chart) with certain behaviors. The father from F3 explains how he could see that his son was playing console at lunch time:

"we can check that he turned on the consoles and the TV, I bet there is a peak in here, he also open the refrigerator door, yeah we can see it...". (F3, husband)

\section{Electricity production}

All the families saw electricity production information as something new and interesting, although some family members already had a rough idea of the available sources. Everybody learned something from the What-a-Watt system, for example F3:

"I knew about the different sources, but I had no idea of their quotas, I thought the solar would be higher that the wind power, I was also expecting more presence of the hydric energy" (F3).

Families relate the source of energy to the infrastructure they encounter in their day-to-day lives, for example everyone predicted the presence of wind power in the local grid, since the wind parks in the mountains are a sight that is almost impossible to miss.

"wind power yes... I've seen the windmill up there" (F4).

The same family was not aware that the islands produce electricity from water or sun,

\section{"I had no idea about the solar power, or even hydro".}

Similarly, families predicted that the sources that are more "visible" were responsible for a bigger part of the island total quota as the daughter from F2 argues:

"We have all those windmills up there... I was expecting that they would be more used... I had no idea it was so little" (F2, daughter, referring to a particular day with less than $10 \%$ of presence of wind power). 
Families expressed concern about the source of the electricity, they were aware that the origin of energy can influence the level of pollution.

" of course its something that concerns us... we know that we should use our natural resources correctly".(F4).

Being in a closed ecosystem also made families aware of the cost of the energy, not their monthly bill, but the cost for the region since all the energy is produced on the island, as the father from family F3 explains:

"Of course if it was all hydro, and wind power it would be ideal... because of the pollution... but also cost for the region it would be much better all wind hydro and solar "' (F3, husband).

All families interviewed were surprised to learn that the price of electricity is the same even if it comes from different places. Therefore, after becoming familiar with the different sources, family members proposed several approaches to maximize the use of renewable sources. For example, the husband from F5 proposes having two prices for non-renewable and renewable:

"I don't think it is fair to pay more for an energy that costs less to produce that the other... I would even say that I would prefer to pay a little more for a non-pollutant energy because maybe that way I would be encouraging a stop in the more pollutant energies production". (F5, husband)

In general families understood that the investment in renewable energy made by the provider has to be covered by someone, but they found it hard to understand that they were paying more for pollutant energy especially if the renewable presence is significant. As the husband from F5 pointed out:

" if you tell me that there is 50\% thermal and 50\% renewable in the grid... in that case it is not fair paying a higher price for a pollutant energy... I would even prefer to pay a little bit more at knowing that my consumption does not have a cost to the environment." (F5, husband)

What-a-Watt also allowed participants to relate the energy produced on the island with variables such as weather or time of the day, as the husband from F5 stated:

"I have that impression, if there is nice weather the solar energy increases.. if it's raining it's bad for solar power". (F5, husband) 
This concept was grasped rapidly and some families could even predict the future energy sources available based on the weather forecast, and this could work the other way around, predicting the weather based on the available sources as stated by F4

"Sometimes I checked that there was some wind power, but I didn't notice any wind around here, I'm sure that it was windy in the mountains'.

None of the families mentioned changing any routines based on the available energy sources. Their busy day-to-day lives make it harder to plan that way. F4 mentions that:

"If I spent more time at home, but I'm only at home after 19:00, and I have to do my stuff around the house, it is complicated to turn on the appliances just because now there is more wind energy". (F4)

The adjustments in family routines were more likely to be made as a result of the cost of energy, and by the eco-feedback device that made that cost visible than motivated by the source of the electricity, as the mother from F5 mentioned:

"turning on plugged in devices, and reduce the usage of the microwaves... it was mostly because of the cost". (F5, wife)

The use of electricity was experienced as something completely opaque from a consumer point of view, as the father from family F3 mentioned:

"when we turn something on, we don't even think where does it come from ... maybe we should". (F5, husband)

\subsubsection{Discussion}

Our eco-feedback device proved to be an easy tool to understand and incorporate into the consumers' routines. We argue that the portability of the device itself, together with the overall robustness and reliability of the hardware-software platform allowed us to offer consumers an experience close to the one offered by commercial solutions. This was possible because our research platform was already tested in other situations and the result of an iterative development process.

The What-a-Watt system helped families to reduce their consumption when compared to the baseline data and the control group. Our results are in line with what literature reports for ecofeedback devices, however we believe it is important to mention that in our studies the participating 
started a decrease tendency during a time of the year when the overall consumption tends to increase, (Autumn and Winter) as it was confirmed by the increase in consumption in all the control group houses and by significant difference between the consumption in the study and the control group.

Even though there was a steep decrease in the usage of the feedback after the first weeks of the deployment, the exploration made in the first weeks made users more aware of their normal routines. After that period the eco-feedback was used more as a tool to check "if everything was ok" and detect abnormal consumption patterns in the household. Additionally, the eco-feedback allowed a tighter control of action that were already being performed by certain elements of the family but were harder to track.

Families considered the energy production information as something new and interesting. We argue that our system helped consumers correct several misperceptions of the electric grid making them more knowledgeable about the long-term implications about energy consumption. The size of our sample does not allow us to generalize our findings, however we argue that the information about the source of the electricity is a valuable addition to eco-feedback devices or even the proposed smart meters. Furthermore, we hypothesize that if a closed system like a small island where the infrastructure used to produce electricity (i.e. windmills, thermal stations or photovoltaic installation) are clearly visible by citizens these misperceptions exist, they are to be bigger in larger metropolitan areas where the majority of the electricity comes from outside the residential and commercial area of the city.

Regarding our goal of bringing the awareness and knowledge of micro-producers to "regular" consumers, we consider that the What-a-Watt system achieved this goal. Our interviews disclosed that consumers rapidly grasped the basic concepts of the grid, and were aware of how variables such as the weather or time of the day affect the electricity distribution. Besides the cost, the source of energy might also be of value for consumers when making decision between tariffs or providers.

Even though all families showed concern and awareness of the source of their electricity, none referred any change in their routines to maximize the usage of a particular energy source. Families mentioned that the lack of time was the biggest hurdle to perform an adjustment. In fact, our analysis of families' routines disclosed that the appliance usage is mostly centered in a period of time (evening/night), where for example the presence of solar energy is virtually zero. Furthermore, considering that $84 \%$ of the events are triggered by low consuming appliances the level of control and alertness necessary for an individual consumer, to shift the consumption to include more renewables is simply unfeasible in one day-to-day routines. Therefore, propose that to overcome challenges such as consumers' lack of time, a system such as the What-a-Watt could work with 
automated agents in the household allowing consumers to trigger certain appliances (e.g. washing machine, air conditioner) when a defined threshold of renewables was met.

\subsubsection{Conclusions}

The What-a-Watt integrated both household energy consumption and real-time production breakdown information from the grid. Our goal was to explore a recent trend were micro-production users showed increased interest about energy production and consumption. This research took that approach further integrating whole grid information from a 270000 island closed network with a high penetration of renewables. Taking advantage of this living lab scenario we are exploring future scenarios where this information could become available to wider network hopefully leading to more informed and sustainable scenarios of energy consumption and awareness.

Our results show that consumers quickly became aware of the raw energy used to produce their electricity. Furthermore, consumers were able to connect variables such as weather and time of the day to the availability of the different electricity sources. Regarding consumption, on average consumers with the What-a-Watt system managed to reduce their energy bill, inverting the tendency to increase in consumption that was observed in the control group.

Our results are also consistent with the findings in medium to long-term eco-feedback research showing a substantial decrease in interaction after an exploration phase of approximately four weeks. Again we observed that after this period the users' attention towards eco-feedback devices will consistently drop to marginal values only attenuated unusual circumstances, like different patterns or the acquisition of a new appliance.

After many years of research, it seems clear that eco-feedback is not the "silver bullet" for sustainable energy consumption. Our unique setup is another contribution for future scenarios where energy (or the consequences of excess energy demand) will no longer become sustainable. In such a future scenario energy production could evolve into new service-oriented models where end-users and consumers will have an important role matching consumption patterns with the availability of renewable energy. 
Research Studies 


\subsection{Watt-I-See: An interactive installation to probe eco-feed- forward strategies}

After the deployment of the different eco-feedback systems described in the previous sections, we planned to explore the connection between consumers and their energy consumption further.

The Watt-I-See (WISE) study combines the findings from our previous four studies. Firstly, we draw motivation from our shift the feedback paradigm present in the SinaisFromFanal and WattsBurning systems (sections 4.4 and 4.5), this way WISE displays the individual's impact in the environment as the main feedback modality. Secondly this study also builds upon the results from the WattsBurning in my Mailbox study in which the feedback is shown in a tangible and public mode.

Finally, our work with WISE also drawn motivation from our study with the What-a-Watt system presenting individuals with the actual raw elements used to produce their electricity. This rationale is explained in detail in the following sections.

\subsubsection{Motivation}

Although eco-feedback devices are nowadays an important tool to save money and control household routines, their role in the household is likely increase once families' energy needs began to be met by a renewed grid. The new grid technologies combine distributed generation, higher penetration of renewable energy, micro-generation, batteries and vehicle-to-grid techniques. In this future scenario both utilities and consumers will have to adapt to the new situation. In particular ecofeedback devices will possibly need to include new information and services such as appliance automation, dynamic pricing information, batteries and renewable energy availability or ecofeedforward among others. We argue that those services will require higher involvement of users than the current state of the art eco-feedback systems.

For these reasons we designed, implemented and studied Watt-I-See, an installation where individuals are presented with the real time resources used to produce their electricity. We take advantage of Madeira's closed grid where all the electricity is produced locally and where it is possible to trace in real time quotas of thermal, wind, hydro and solar electricity in the grid. With this advantage at hand, we envision future scenarios where the energy consumption might have to adapt and plan according to the production at each moment. 
WISE design decisions combines the work from two distinct eco-feedback sub fields. Firstly, we motivated by studies that presented consumers about the source of the energy, and studies that have displayed the effort needed to produce electricity it in the feedback (see section 3.5.4 of our background review). Secondly this study, like the WattsBurning on my mailbox study (section 4.6.1), takes advantage of tangible interaction to display information that is normally hidden and not clearly relatable by individuals, furthermore the inherent educational qualities of tangible interaction motivated us to also evaluate study WISE with children.

This chapter presents the two studies performed on the Watt-I-See system. One was performed with adults in which we which we attempt to move beyond the known limitations of current ecofeedback systems and enhance the understanding of how consumers experience energy production. The second study was performed with a group of children. Through this study we probed children's awareness regarding renewable energy resources and their interaction with the WISE system.

We planned to conduct two studies with different demographics since both adults and children play an important role in the household dynamics relating energy consumption. Adults are normally responsible for buying the energy consumption appliances and paying the bills. Children were included in this study since research showed the importance of the younger demographic in the household as parents try to educate their children (Barreto et al., 2014; Quintal et al., 2013).

\subsubsection{Watt-I-See raw data input}

In our setup we explore the information of Madeira's larger ecosystem consisting of 270 thousand inhabitants in which the grid operates in a closed circuit system. The island's electricity needs are fulfilled by two thermoelectric power stations, four wind parks (located in the mountains), 10 hydroelectric stations (near the base of the mountains), and approximately 700 small and medium sized photovoltaic installations (dispersed) (more about location at section 4.1). The average yearly electricity production is distributed as follows: $78 \%$ of all energy is produced from thermoelectric plants, $11 \%$ from hydroelectric stations, $9 \%$ from wind parks and $2 \%$ from dispersed photovoltaic sources. During winter months the wind and hydro energy source quotas increase significantly, while during dry summer months the renewable quota is significantly lower. The local electricity production is obtained through the process described in section 


\subsubsection{Visualization}

Watt-I-See resorts to an analogy of "x-raying" a household wall, exposing the invisible content carried inside pipes and cables that we do not normally get to see. In order to re produce the pipes metaphor we resorted to the use of four glass pipes containing a colored vortex, each representing an energy production source available locally: thermoelectric power stations, wind parks, hydroelectric stations and photovoltaic. The metaphor conveys the idea of different types of energy fuelling our household, giving us access to the knowledge of the breakdown and proportions of renewable energies versus fossil.

The glass pipes contain distilled water and liquid coloured paraffin. Each vortex is colored in order to represent a different energy source: dark purple for thermal energy, clear colour for wind, dark blue for hydro and yellow for solar energy source. The size of the vortex, ranges from very low (between 1 and 3\% quota) to maximum (these nine levels represent the quota in percentage. The size of the vortices is measured from the top of the tube $(0 \mathrm{~cm})$ to the bottom $(30 \mathrm{~cm})$. The highest level creates a more aggressive vortex to represent over $91 \%$ (limit selected by average maximum thermal quota) of quota from an individual production source. These levels represent the quota of energy produced and available to final consumers, thus the sum of all four vortices totals $100 \%$ (See Figure 39).
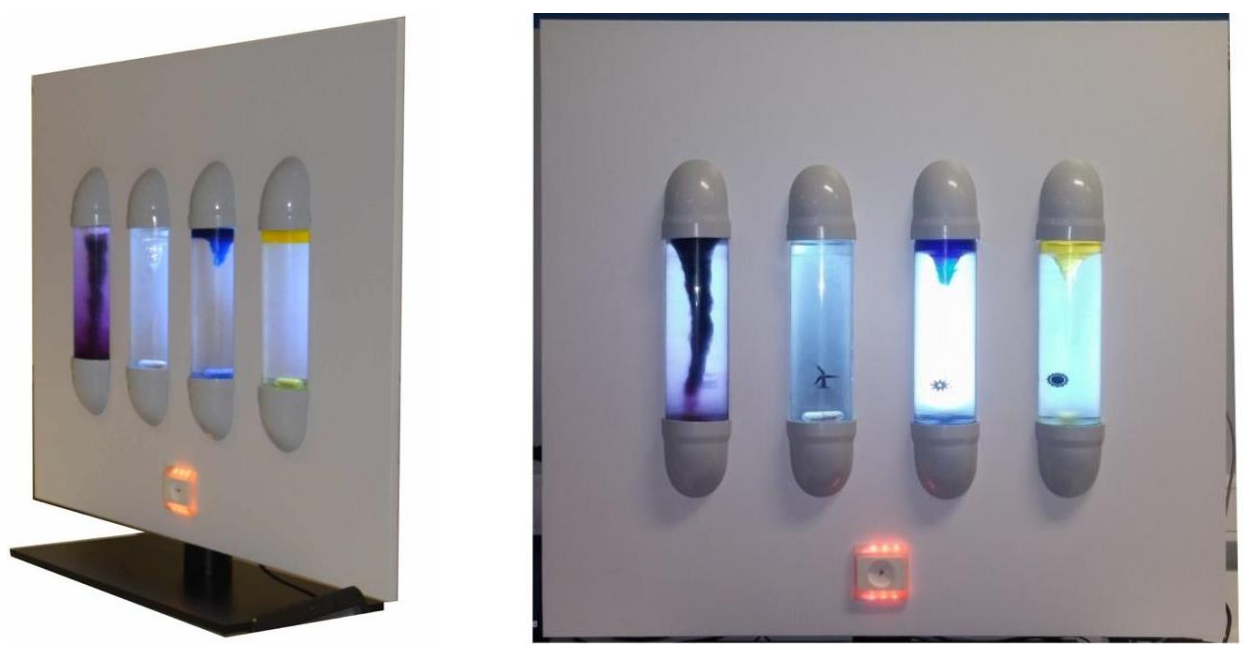

Figure 39:WISE panel displaying low presence of renewable energy. 


\subsubsection{The Glowing Power Socket: Renewable Energy Feedback}

In addition to the vortices, the installation is provided with an electricity socket, which was named power socket. The power socket provides additional feedback on the overall quota of renewable energy in the grid. While the glass tubes and vortices display individual source production quotas, the power socket displays the cumulative quota of renewable energy in the power grid. Five renewable feedback levels were defined based on three years of disaggregated production quotas from red (high percentage of fossil usage) to green (high percentage of renewable energy usage). Each level displays on the power socket a corresponding pulsing colour (See Figure 40).

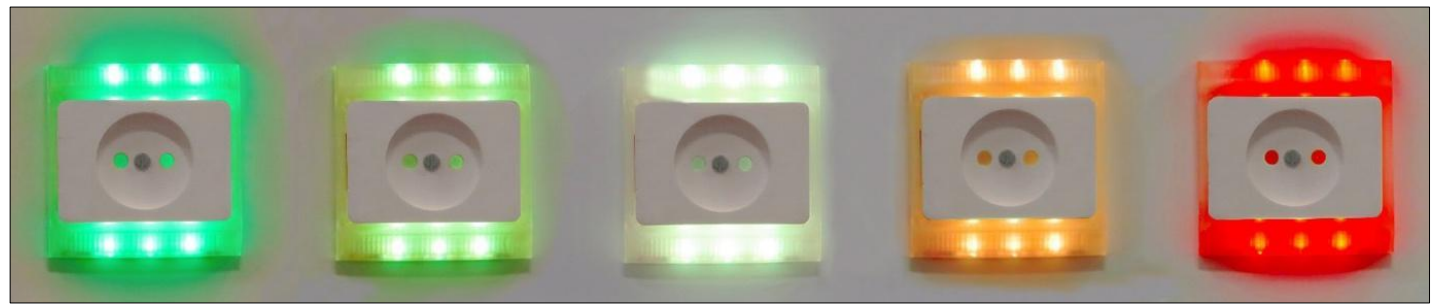

Figure 40: Five possible stages of the Power Socket, from high presence of renewable energy (left), to low presence of renewable energy (right).

\subsubsection{Production Visualization}

Watt-I-See measures $1.22 \mathrm{~m} \times 0.9 \mathrm{~m}$ (without the stand) built from wood and covered in matte white vinyl. Four glass tubes $(9 \times 30 \mathrm{~cm})$ are used to represent the individual production sources. Vortices are created by DC motors controlled by an Arduino microcontroller. The DC motors rotate a large magnet that subsequently rotates a magnetic bar inside the glass tube creating the vortex. A smaller magnet and Hall effect switch is used to calculate the rpm's of each motor in order to leverage the rotation between the different tubes. Additionally, LED strips and drivers are used to retroilluminate the glass tubes that are covered in tracing paper and contain the printed icons for each energy source (See Figure 40). A computer power supply is used to power the installation.

The electricity socket is a common power socket where the outer bezel was 3D printed using a transparent plastic. The color of the socket is obtained by RGB LEDs and is based on the outcome of a model which includes several conditions queried in real-time: 1) current real-time production quotas; 2) current day averages; 3) week averages; 4) month averages; 5) five-hour prediction quotas.

An application built in Processing communicates with the Arduino microprocessor. This application is responsible to start/end the installation as well as changing the presentation displayed. 


\subsubsection{Interaction}

The WISE installation was evaluated with two different groups of people, adults and children, therefore the actual interaction with the installation was different among the groups. However, the logic behind the interaction was the same, individuals (both children and adults) selected weather and time of the day variables which would then affect the presentation in the WISE vortexes and power socket.

\subsubsection{Interaction rationale}

The rationale behind the interaction with WISE originated by the fact that in general consumers cannot control what energy is produced at each moment. At the very best a consumer can predict the weather or other natural phenomena associated with the availability of energy (Chetty et al., 2008; Filonik et al., 2013; Woodruff et al., 2008). This way in the WISE interaction consumers can control several variables related to the time of the day and weather conditions that would affect directly the installation

In both evaluations after a selection was made, the installation would need approximately 10 seconds to adjust before settling in the correct visualization. Next we explain how the interaction with WISE was adapted for children and adults.

\subsubsection{Adults interaction with WISE (EV1)}

Adults were given a set of cards (See Figure 42) in which they combine the following variables: rain (4 values) wind (4 values), cloud coverage (4 values) and time of the day (6 values), participants would then select a combination of the cards and a research member would input those values in a Processing application. This application would then query our database with 2 years' worth weather conditions for the island and select a date where the conditions matched the selected cards (or at least the best fit). Next, the application queries our database with real production data for those two years and collected the production quotas for the selected day, those values were finally used to update the WISE panel and power socket.

\subsubsection{Children Interaction with WISE (EV2)}

Children interacted with the installation in a simplified manner. Children interacted with the WISE installation by manipulating a set of six small Lego ${ }^{\mathrm{TM}}$ blocks, placed in front of the installation, 
see Figure 41 right. The blocks were used to manipulate weather conditions, stacking them on top of the three icons placed on the table representing, sun, wind and rain. The sun, rain and wind variables could have three different values (low, medium and high) resulting in nine possible combinations of weather conditions (see Figure 41 below). The selected weather conditions influenced directly the displayed energy quotas (rain hydroelectric, sun photovoltaic and wind affected wind power). The first Lego ${ }^{\mathrm{TM}}$ block placed on the corresponding weather icon represented $5 \%$ of the total energy production, while the second stacked block represented an additional 15\% (by itself) and the third one an additional $25 \%$ of the selected energy, for a maximum total of $45 \%$ per source. The fossil- fuel energy quota was used to fill up the remaining until $100 \%$ of energy production. For example, one Lego ${ }^{\mathrm{Tм}}$ block placed on the sun icon and two blocks on the rain icon would result in $5 \%$ of photovoltaic energy and $20 \%(15 \%+5 \%)$ of hydroelectric energy produced on the island, while the rest was filled by thermal energy (75\%). If the weather conditions would sum up for more than $100 \%$ we explained to the children that such conditions were impossible to happen all at the same time in real life (for example, lots of wind, rain and sun simultaneously).
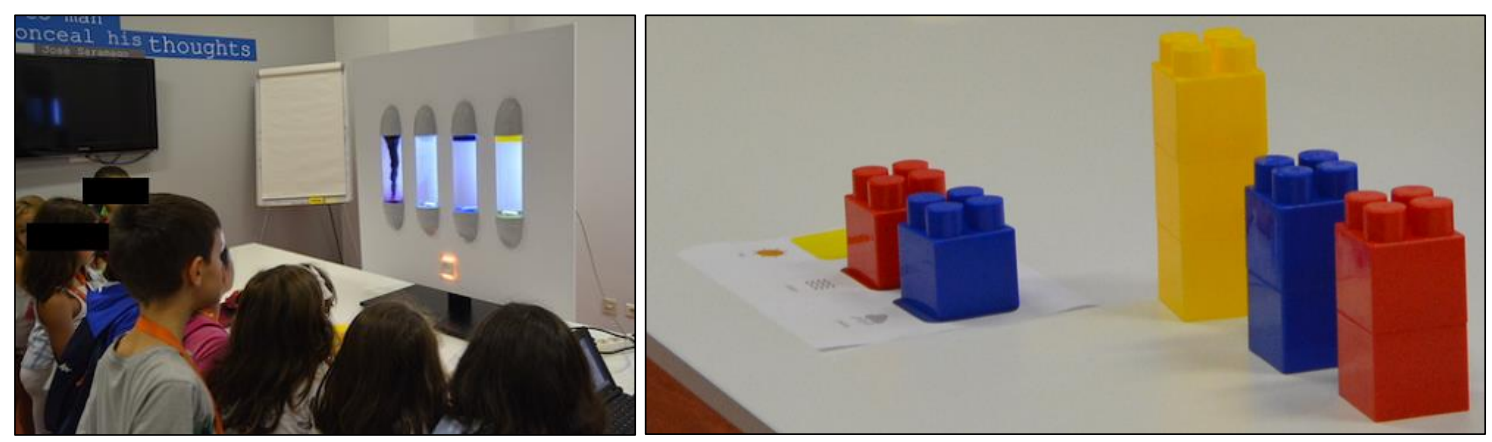

Figure 41: Left: Children observing the WISE's visualization; Right: Legos used in the interaction with children.

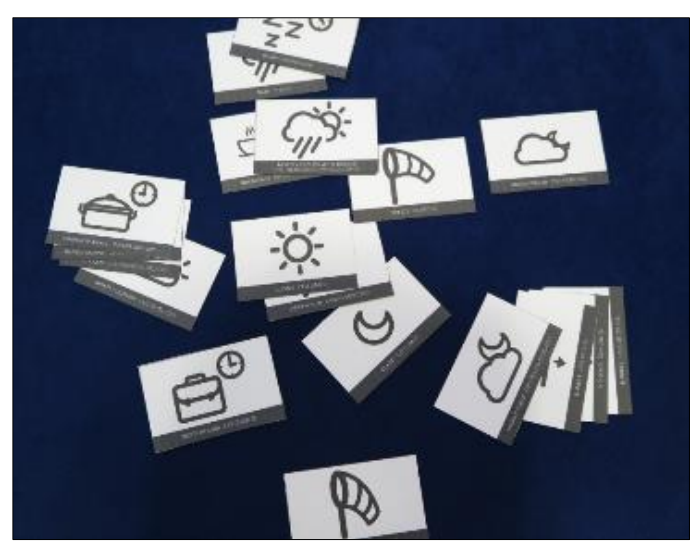

Figure 42: Card used to manipulate WISE by EVI 


\subsubsection{Evaluation}

The WISE installation was evaluated in two isolated events. Ten adults and fifty-six children interacted with the application in two distinct moments. In this section we describe how both evaluations proceeded.

\subsubsection{Adults evaluation (EV1)}

The first study started with a questionnaire with 57 electricity consumers, 35 male and 22 female, with an average age of $29(\mathrm{M}=29, \mathrm{SD} .=8)$, from an Island in the south of Europe.

Participants were recruited through social networks and word-of-mouth. They were asked to complete an online survey that assessed individuals pro environmentalism, through the New Ecological Paradigm scale (NEP) (Dunlap et al., 2000) and measured individuals electricity production literacy and behaviours.

Apart from the standard 15 questions of the NEP, the survey contained six multiple-choice questions regarding the local production of electricity, two multiple-choice questions relating to individual behaviours towards electricity consumption, and four questions where consumers were specifically asked to list and rank the local energy production techniques used. In the analysis of the questionnaire, we considered three sets of possible answers: correct; incorrect; and answers that only missed by one choice (only missing one production quota position incorrectly still represents a high level energy literacy). The questionnaire used in EVI is presented at Appendix 9.8.

\subsection{Sample EV1}

Ten responders of the survey were selected to undertake the remainder of the study and interact physically with Watt-I-See at the regional Science Park. Each participant interacted with Watt-I-See individually for the duration of approximately 50 minutes. The study included free interaction with Watt-I-See, a 14 question semi-structured interview (Appendix 9.9) recorded through the researcher laptop and a 22 question (five-point Likert scale) survey at the end (Appendix 9.10). The study ran under the supervision of two researchers: an interviewer and a note taker. Audio of the interviews was recorded. 


\subsubsection{Children evaluation (EV2)}

The second evaluation included a formal evaluation of children's knowledge concerning energy sources through a five-question questionnaire conducted after the interaction with the WISE installation (the questionnaire is presented at appendix 9.11). Five questions tested the children knowledge about the local electricity production, the impact of different sources of energy on the environment and how the weather conditions impacted the electricity produced locally. One question probed the children about how enjoyable was the interaction with WISE.

The questions were constructed taking into account the limitation that small children might have when answering questionnaires (Read and Fine, 2005). This way all questions had graphical and textual representations for the answers and a psychologist member of the research team read the questions out loud and monitored the children along the way. Table 5 lists the five questions presented in the questionnaire. Throughout the rest of this document this questions will be referred as Q1, Q2, Q3, Q4 and Q5 respectively. The questionnaire was previously validated with a different group of four children with ages between 8 and 13 years old.

Two multiple choice questions (Q1 and Q2) asked children to select the types of energy produced locally and the types of electricity generation techniques which used renewable energy. Q3 inquired children about which production techniques are good or bad for the planet. Q4 presented children with three possible weather conditions (windy, sunny and rainy). Children were then asked to connect representations of the weather to pictures of four electricity generation techniques used in the Island. Children could assign one or more generation techniques to each weather condition based on how much that particular technique influenced the overall local load. Q5 presented children with a timeline of a day with different weather conditions. Children were asked to connect three points (morning, mid-day and night) to one or more of four electricity production techniques (see Figure 43). Table 5 lists the five questions in the questionnaire.

\subsection{Sample EV2}

Fifty-six children visited our research Institute facilities during which visit they could try different demos, games and see exhibitions of research work, as well as interacting with the WISE installation. The evaluation of WISE installation was performed during such visit. To facilitate interaction, the fifty-six children (average 8.4 years old, 25 males) were organized in six groups based on their age:

- Group A: 7 kids between 10 and 12 years old 
- Group B: 7 kids between 10 and 12 years old

- Group C: 11 kids between 8 and 9 years old

- Group D: 11 kids between 8 and 9 years old

- Group E: 11 kids between 6 and 7 years old

- Group F: 9 kids between 6 and 7 years old

The interaction with WISE was evaluated using a Wizard of $\mathrm{Oz}$ approach (Dow and MacIntyre, 2007). Each group had approximately 10 to 12 minutes with the installation followed by 10 minutes to fill in a questionnaire. Both the interaction and questionnaire sessions were video recorded. Figure 43 shows the setup of the experiment. WISE was placed in a table and surrounded by participants, the Lego ${ }^{\mathrm{TM}}$ blocks although not visible in Figure 41 (left) were placed directly in front of the WISE panel.

Table 5: Five questions used in the evaluation with children

\begin{tabular}{c|c} 
ID & Question \\
\hline Q1 & Select which energy sources you think are available in the Island: \\
\hline Q2 & Select which energy sources you think are renewable: \\
\hline Q3 & Which energy sources are good and which are bad for the Earth? \\
\hline Q4 & Which energy sources are available when its rain/sun/wind...? \\
\hline Q5 & Select which energy sources are available during the day
\end{tabular}




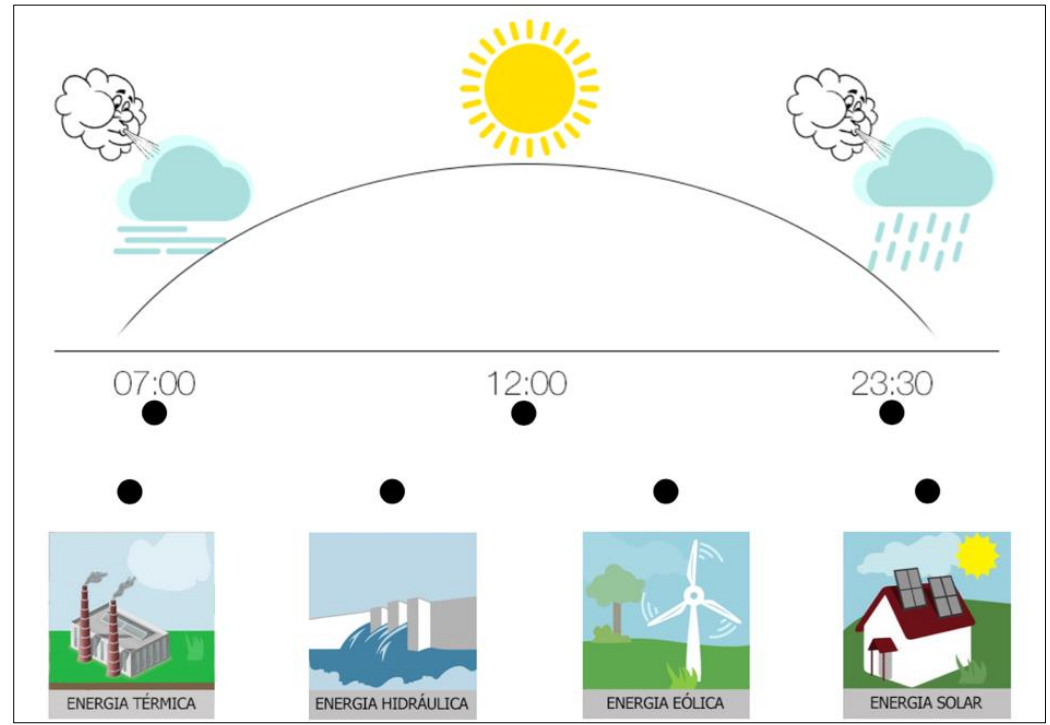

Figure 43: Fifth question of the children's evaluation of WISE.

\subsubsection{Results}

In this section we will summarize the results from both evaluations. Results will be organized in themes extracted of the qualitative assessment of both studies. Whenever it is relevant quantitative results will be added to this summary of the results. Also, whenever we found relevant quotes were added to the results. For EV1 participants will be referred as P1 to P10, for EV2, since the evaluation was done in groups it was not possible to isolate individual reactions, this way the quotes from a participant in EV2 are unidentified.

\subsubsection{EV1}

\subsection{Usefulness of WISE}

The qualitative evaluation with adults was more focused on the information shown by WISE and how that information could be used in an eco-feedback device. In general adults' reactions to the system were pragmatic as they tried to gauge the value of a system like WISE could bring to their own energy consumption monitoring strategy. All of them agreed on the usefulness of WISE. Seven participants commented how WISE information helps understanding one's impact on the environment. This observation was more evident when participants discussed about the power socket, six participants affirmed that if they had to choose one functionality from WISE they would 
chose the coloured power socket, due to its location at the point-of-interaction, where the consumption activity is happening. Participants also referred that the power socket provides enough information to make a decision (based on the colour feedback), it's easy to understand and immediate, attracts attention and summarizes the disaggregated information from the tubes in realtime.

Regarding the information displayed it was observed that participants had in general a wrong preconceived perception of the quotas of each energy used in the island, this way the interaction with WISE clarified individuals' understandings. Eight out of 10 participants expected solar power production to be higher. P10 commented how the island has a lot of solar panels and how on average it's a sunny place:

\section{"I thought solar was higher since there is no need to direct sunlight to produce photovoltaic energy"}

Six participants expected wind power to be higher, again comments based on visual awareness as they relate the production directly to the high number of wind turbines existent in the island. Overall, for some sources such as hydroelectric, participants commented on how very little they know about it, and how it is uncommon to see a hydroelectric station. As one P3 referred, hydroelectric power might not be related to the "now", but heavily influenced by passed weather conditions such as, if has been raining for the past days:

"I was not expecting the water energy ... I did even know we took advantage of enough hydro electricity to feed into the grid ... I thought it was insignificant"

In conclusion, during the interview 5/10 participants expected a much higher production of renewable energy. Like P6 commented

"The others (renewable vortexes) one are so low they should be used more often ... the fact we do not use more natural resources ... I was expecting more"

\subsection{Presentation of the production information}

Participants found the presentation of the data through the WISE interface itself attractive, easier to understand and provided a good notion of what is going on as it was fast to consult and intuitive. 
Six of the ten participants liked the colours representing each energy source, the movement of the vortex and how water closely relates to energy and the evil colour of the fossil energy source vortex. Three participants described WISE as more visible and "realistic" commenting on the movement and water vortex appearing like energy or something powered by energy, Like P7 from EV1 mentioned.

"Numbers are not as memorable... if you asked me the numbers of my last electricity bill I cannot remember... I would more likely remember this"

However, six participants mentioned how it was less precise than graphs and numbers.

Participants also suggested different additions to the installation that would help them get more information: a projection of resources to be used to produce energy; a version of WISE to be used in a mobile platform it was also referred that the same information could be displayed through bars of graphs to be easier to consult, as $\mathrm{P} 2$ suggested:

"It would be interesting, if we could have this in the smartphone like a widget with the production of each one of the different energies, maybe using symbols or a bar chart to present the relationships between the quotas"

\subsubsection{EV2}

The evaluation with children was more focused on the interaction with WISE, and how they used the Lego ${ }^{\mathrm{TM}}$ blocks to manipulate the weather conditions that would then influenced the presentation.

\subsection{Interaction with WISE}

In general, when children interacted with the installation, they had frequent verbalizations with the researcher exploring scenarios and commenting on what they observed in the installation:

"Let's have more of this..." or "Can we have one of each?" or even "If we remove it becomes smaller/bigger”.

The selection of the Lego ${ }^{\mathrm{TM}}$ blocks to control the WISE display was made collaboratively through frequent verbal interactions between the children closer to the installation and the ones further away. Overall each group tried around six to eight different combinations. Children took turns interacting with the blocks and selecting a combination. 


\subsection{Understanding of the energy sources}

We also assessed how the interaction with WISE affected children's knowledge of the local electricity production process, this was done via the post study questionnaire. Next we present a summary of the questionnaire results.

Before analysing the questions individually, the count of correct answers was normalized (since certain questions have more correct answers than others). Table 6 summarizes the results of the questionnaire, in the following we discuss each question answers in detail. In Q1 the most selected sources of energy in the Island were solar energy (53 out of 56 answers) and wind energy (45 out of 56 answers). The most common misconception was the absence of fossil-fuel (35 out of 56 ) and biomass generation ( 31 out of 56 answers) from the set of available local sources of energy.

In Q2 the two most frequent accurate answers were selecting solar as a renewable energy source followed by not selecting nuclear (47 and 46 out of 56 answers respectively). The most common misperception in the question was not to mentioning biomass and hydroelectric energy as renewable energy sources ( 27 and 35 out of 56 answers respectively).

Like we summarize in Table 6 there was a maximum of seven correct and seven incorrect answers to Q3. Fossil-fuel and Nuclear energy sources were selected, 52 out of 56 respectively, as bad for the planet. The most common misconception was selecting biomass and hydroelectric as bad for the environment ( 9 and 10 incorrect answers out of 56 respectively).

Considering Q4 the most common misconception (71\% of the answers) was not to add the fossil-fuel energy option too all days, since there is always a baseline (which sometimes is quite significant) of fossil-fuel energy produced locally.

Finally, in Q5 (see Figure 43) 87\% of the participants identified midday as the best time of the day to produce photovoltaic energy. In fact, $70 \%$ of children were able to identify the morning as being the best time to produce hydroelectricity, midday to produce photovoltaic and night to produce hydro and wind electricity. The most common misconceptions in Q5 was not selecting wind energy available at night (78\%) and not adding fossil-fuel energy in the morning (66\%). 
Table 6: Average results by question of the post-study questionnaire with children

\begin{tabular}{l|l|l|l|l} 
Question & $\begin{array}{l}\text { Maximum } \\
\text { correct }\end{array}$ & $\begin{array}{l}\text { Average } \\
\text { correct }\end{array}$ & $\begin{array}{l}\text { Maximum } \\
\text { incorrect }\end{array}$ & $\begin{array}{l}\text { Average } \\
\text { incorrect }\end{array}$ \\
\hline Q1 & 5 & 3.03 & 7 & 2.41 \\
\hline Q2 & 6 & 3.03 & 7 & 2.46 \\
\hline Q3 & 7 & 6.08 & 7 & 0.89 \\
\hline Q4 & 6 & 3.76 & 6 & 2.35 \\
\hline Q5 & 8 & 3.8 & 7 & 3.55
\end{tabular}

In general, our observations of children's verbalizations disclosed that they understood the energy sources as some children would reply to other children's' questions or volunteer to explain that fossil fuel was oil. One child even added:

"Oil is bad for the sea, the plants and the environment". Other children reacted to the combination a lot of rain and sun "you have a rainbow".

The actual representation of energy also had an impact in children as they expressed amazement while observing the first demonstration "This is cool!". Children interrupted to ask questions and possible scenarios with "how about this...?" or "what happens if...?".

\subsubsection{Discussion}

In this section we discuss the results from both evaluations, the discussion will be organized in themes we found important throughout the study. Some themes will combine results from both evaluations while others will be unique for EV1 or EV2.

\subsubsection{Usefulness of the production information in the daily lives}

When evaluating WISE with adults it was clear that participants started to think about how to include the provided information in their day to day routines, or how to change their routines to maximize the usage of one type of energy over the other. This way, one of the preferred aspects of 
Watt-I-See was feedback immediacy: The glass tubes and vortices provide just enough information to inform a decision. The feedback power socket is as close to the point-of-interaction as possible, however, possible at times not the most visible due to the location of power socket. On this point, five participants commented on mobiles phones as an "easy to reach interface" that is always present (or near) consumers.

Participants also commented about how WISE's information could be incorporated into other devices, as P2 (from EV1) commented

"it could be interesting, if it was possible on the phone possible on a widget with the production levels of each energy, using symbols and bars to show the relation among them".

It seems that a simple relation between different energy sources and its quotas provide enough renewable information for the average consumer's curiosity.

Adults also hypothesize scenarios of usage of a tool such as WISE, participants referred several constraints that could hinder shifting consumer behaviors according to the available electricity. Some daily or household activities cannot be shifted, like breakfast in the morning or dinner in the evening for example. However, some activities were mentioned as more flexible, such as washing machines and ironing. Yet, these activities are still affected by other factors such as weather conditions, for example using wind and sun to dry clothes naturally or using the washer and drier machine. Withal, sporadic consumption routines were mentioned to be abler for shifting than daily routines. Extrinsic motivators such as monetary rewards from consumption reduction are not expressed through the Watt-I-See feedback. The study seemed to invoke more intrinsic motivators from the participants such as feeling worried or depressed when viewing the fossil vortex at its maximum scale. P6 (from EV1) further reinforces the idea:

"I would probably have only the first tube because it is possible to know what we are doing wrong... while the others are good I do not need to worry about reducing them."

\subsubsection{Energy Visualization}

The visualization of the energy itself also had an impact on participants of both studies, we argue that the dramatic presentation of WISE not only foster curiosity but also impacted the way participants viewed the information. 
Children (EV2) saw the installation as something cool, they competed to see which one could interact with it. The ones that were not interacting with the installation were giving suggestions to their colleagues or commenting the results. In summary our study showed that children enjoy tangible interaction when learning about complex concepts such as the correlation of naturally occurring phenomena and renewable energy. Results from our study suggest that WISE could work well as a complementary tool to science and energy education in schools. Not only to reinforce learned concepts (sun and wind as sources of renewable energy for example) but as well to unveil the hidden or unclear ones (biomass and fossil-fuel energy baseline).

Regarding the adults (EV1), WISE was defined as realistic representation of energy (P5, P9 and P10). Movement showed a significant effect on the engagement of participants. As participant P3 explained:

"...I like the colors [of the vortices], I like the fact that it is a whirlwind because it has to do with energy something always in movement, being produced (...) people need to know that it is energy and that energy manifests itself in many ways...it is something in movement or puts in movement."

As a result, the simplistic representation of the energy production quotas thought the size of the colored vortex allowed for an attractive, understandable effect, that was suggested to be faster to consult and more intuitive.

A stronger impact at times was noted. Participants felt somewhat worried when viewing the fossil vortex at its maximum scale how aggressive it looked, "that looks worrying" commented a female viewer when she realized that there was approximately $85 \%$ of thermal (fuel production) energy being produced. Other commented on the difference between fossil and renewables as depressive, something as needed to be dealt with.

\subsubsection{Interacting with WISE}

Considering the EV2, the experiment setup enabled up to nine different weather combinations, each group tried on averaged six different possibilities. This suggests the tangible interactive installation was highly successful in promoting exploration between naturally occurring phenomena and the availability of different forms of energy. This result is in line with the body of work which supports tangible interaction as a pedagogical tool (Antle et al., 2009; Bakker et al., 2011). We argue that these observations are justified by the tangibility and the familiarity of the raw resources used to produce renewable energy, and by the "learning by doing" inherent quality of tangible interaction 
(Bakker et al., 2011; Zhang et al., 2010). Which in this case benefited the learning of variables that were more directly related to the tangible manipulation and detracted those who did not.

Although to a lesser extend adults (EV1) interaction with WISE was also valuable, in fact 9/10 participants felt confident they could estimate the availability of renewables based on time of day, season and weather conditions after the study.

\subsubsection{Impact of production information.}

Both adults (EV1) and children (EV2) were surprised when first exposed to the production information, as it defied pre-existent notions of what kind of energy is available in the local network. As P3 from EV1 commented:

"I though the sun [solar energy] would be stronger [higher quota]...the island has a lot of solar panels...and a lot of sun (...)”.

Similarly, P6 also from EV1 commented for wind power:

"we could have also a bit more eolic [higher quota for wind power] since we have so many [wind turbines] is could be put to better use."

For less "visible" renewable source productions such as hydroelectric consumers were mainly unaware of its impact in the grid. This observation is constant in both groups and we can argue that the visibility and everyday presence of the infrastructure used to produce solar (PV panels on the rooftops) and wind (windmill at the top of the mountains) electricity made participants overestimate its presence. On the other hand, hydroelectric stations are positioned in the base of the mountains away from residential areas, and this might be the cause for participants' misperceptions regarding hydroelectric energy. This argument is also valid to justify the overestimation of renewable energy in the island, since the infrastructure used to produce thermal energy is in an isolated place not accessible to "normal" citizens.

Another misperception both in children and adults (EV1 and EV2) was an overestimation of the presence of renewable energy in the grid. Adults were expecting the natural resources were more used in the island, like P5 from EV1 mentioned:

"Since there is no wind I was expecting the wind power to be higher".

Children, also overestimated the renewable quota by not mentioning the presence of a baseline of thermal (non-renewable) energy in the grid. This observation was clear from the answers of Q4 
and Q5, which indicate that children do not understand that fossil-fuel is always used (at least locally) regardless of the penetration of renewables. More than two thirds of respondents (67\%) failed to identify this phenomenon. This is of course related to the fact that fossil fuel is used to fill up the baseline of energy not met by renewables and not directly related to the naturally occurring phenomena manipulated by WISE. However, this is also how the electricity production process works and children (like probably many adults) totally miss this important consequence of energy production networks.

\subsubsection{Conclusions}

With WISE we explored a possible future for eco-feedback devices through an installation that presented individuals with the origin of their electricity. WISE was developed as a probe which we used to evaluate how adults and children perceive electricity and the electric grid. Both groups found value in different parts of WISE, adults were more pragmatic and suggested ways of including production information into their routines, on the other hand, children were more curious of seeing the visualization in different stages and engaged in the visualization itself.

Another common observation was an overestimation of the renewable presence in the grid, children were completely unaware of the thermal energy baseline which is always present, while adults thought the natural resources in the island were used more. Another consistent observation was a general unfamiliarity of hydroelectricity in the island. We connected both observations with the physical presence of the grid infrastructure in everyone's lives, which might have caused both adults and children to overestimate its presence in the total quota.

WISE's also allowed participants to quickly understand the relationship between the weather and the available energy, and this study is another evidence that energy consumers grasp this concept as the way of connecting to the produced electricity.

Withal our studies have shown collectively that although both children and adults found interest in knowing more about the efforts to produce electricity there is still several misconception regarding the grid operation. Our study exposed consumers to a disruptive scenario to which the electric grid is certainly approaching. Moreover we decided to divide our study into two users studies in order to better cater for two very different target users, adults, who have power in the household and children since research has shown the importance of children in energy savings techniques in household (Barreto et al., 2014; Quintal et al., 2013). 
Regarding the interaction with WISE, we observed that the immediacy of the feedback in the power socket was one the favourite features of WISE, this observation supports literature findings which state that the feedback is the most effective when presented close the time of decisions and point of interaction (Fischer, 2008). Individuals also referred that WISE kinetic representation of energy production helped deliver clear information about the electricity reaching one's home. This observation was true both for adults and children, consequently we believe our approach of displaying the production in an abstract representation achieved its goal allowing us to study individuals' thoughts and reactions regarding the logic behind the electricity consumed in their daily lives.

The findings from these studies indicate promising directions for new forms of eco-feedback, which we can name as Eco Feed Forward. Eco Feed Forward are strategies that control or modify the energy consumption based on anticipated results or effects of usage patterns and availability of renewable energy with the goal of reducing environmental impact. 
Research Studies 


\section{Summary of research studies}

In this chapter we summarize the different deployments and research studies involved in this thesis practical work. For this we use the scale proposed by Froehlich in (Froehlich, 2012) which represents the design-space for eco-feedback systems. It depicts the different dimensions of ecofeedback systems as follows:

- Information Access : how the information is accessed, updated and how attention-demanding it is;

- Interactivity : how much the user can interact with or customize the feedback interface;

- Display Medium : physical aspects of the eco-feedback system;

- Actionability/Utility : how easy it is for users to start with information presented by the system and change their behaviour accordingly;

- Data Representation : how the consumption information is presented to the user;

- Motivation/Persuasive Strategies : persuasions strategies employed with the goal of promoting proenvironmental behaviors;

- Comparison and Social Aspects : how the feedback system uses comparisons to motivate consumers to adopt more pro-environmental behaviours

To better position our different prototypes and contributions we identify our work with respect to Froehlich's eco-feedback design space. Each one of the following tables represent a dimension of eco-feedback design, all of our systems are identified by a circle in the different scales, Figure 44 displays the legend use throughout this classification. Section 3.4.1 presents a detailed description of the abovementioned scale.

\begin{tabular}{|ll|}
\hline SINAIS Power Meter I.0 & WattsBurning \\
SINAIS Power Meter 2.0 & WattsBurning in my mailbox \\
- SinaisFromFanal & WattsBurning \\
\hline
\end{tabular}

Figure 44: Colors used to represent our systems in the classification according Froehlich's design space 


\subsection{Information access}

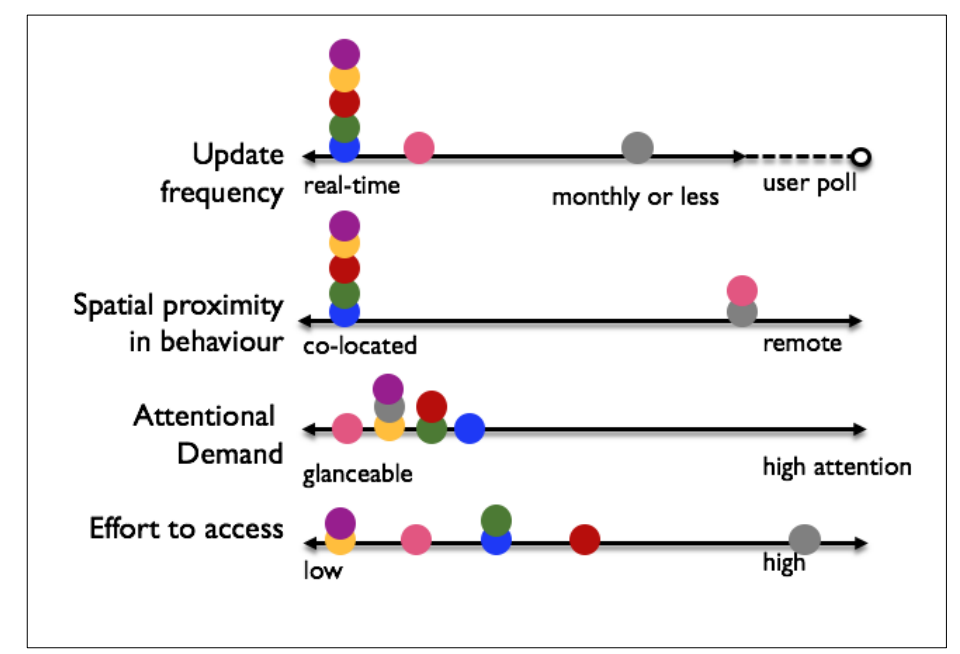

Figure 45: Information access classification of the practical work of this thesis.

As we can see from the Figure 45 in terms of information access most of the eco-feedback systems developed in the context of this thesis work worked in real time in terms of update frequency and where co-located in terms of spatial proximity. Although with different degrees all of the systems provided glanceable attention demand. Our research studies had an access effort from low (WattsBurning and What-a-Watt systems) to high (WattsBurning on my mailbox).

\subsection{Interactivity}

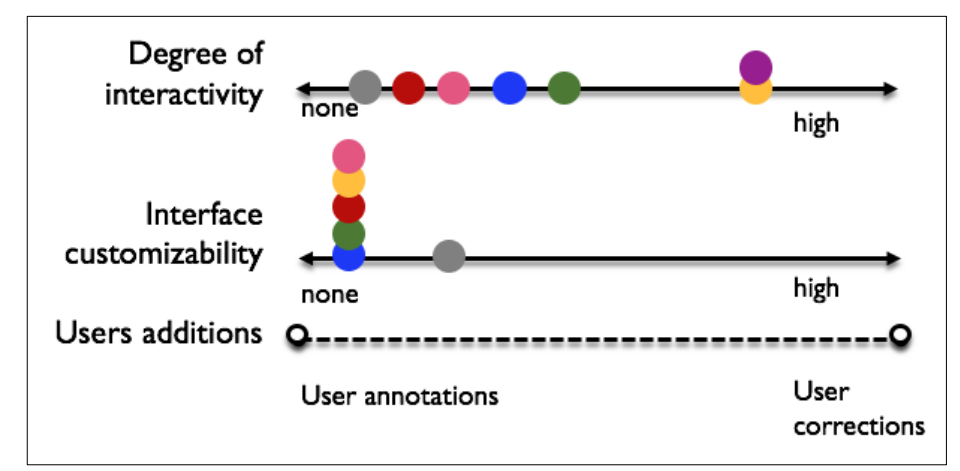

Figure 46: Interactivity classification of the practical work of this thesis.

The scale presented above (Figure 46), shows that our eco-feedback systems offered different degrees of interactivity, ranging from low in the WattsBurning in my mailbox (which was a static set of magnets) to high in the our two last system which ran on a tablet hardware (WattsBurning and 
What-a-Watt). Considering the customization metric, apart from the WattsBurning in my Mailbox study, in which each family had one unique representation of a forest in their mailbox, our systems did not allow customization in the interface.

\subsection{Display Medium}

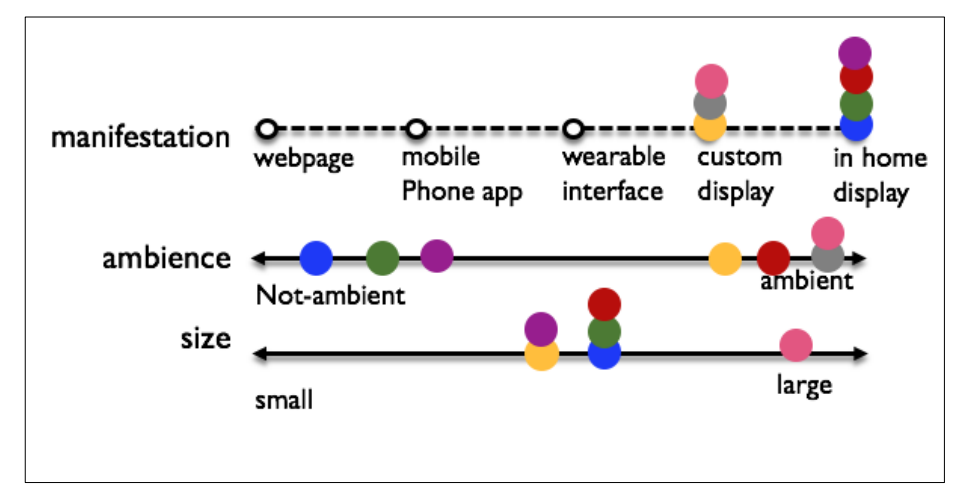

Figure 47: Display medium classification of the practical work of this thesis.

The eco-feedback systems developed in this thesis presented their information throughout different hardware and using different strategies (Figure 47). The WattsBurning in my mailbox and Watt-I-See studies used custom build displays, the rest of the studies where presented in commercial systems either a netBook or a tablet. Our systems are positioned in the opposite sides of the scale regarding ambience features of the interface, in the ambient side there for example the Watt-I-See or the forest illustration in WattsBurning, in the other side of the scale the What-a-Watt and SINAIS Power Meter 1.0/2.0 which presented more concrete data. The physical size of our system was, again, constrained by the physical device holding them (the netBook, tablet and mailbox), this way there were relatively small, on the other hand Watt-I-See installation was significantly bigger 


\subsection{Actionability/Utility}

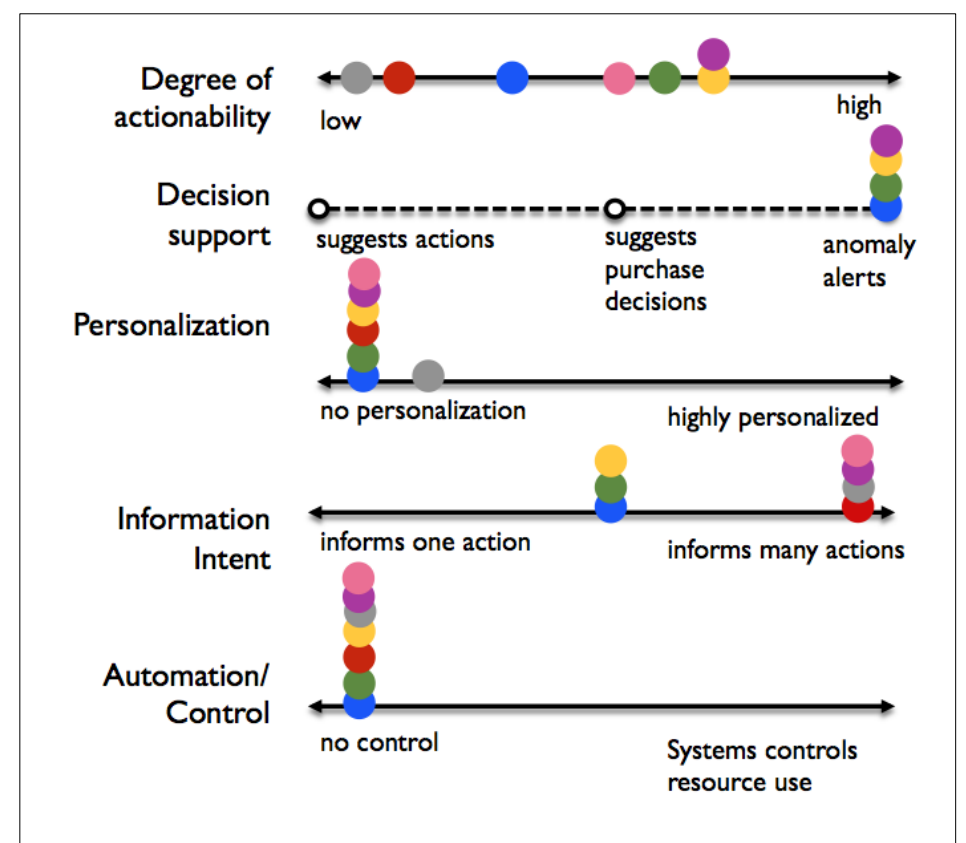

Figure 48: Actionability/Utility classification of the practical work of this thesis.

Figure 48 presents the Actionability/Utility classification of the eco-feedback systems developed in this thesis. The Actionability of the presented information is different across our systems, however the systems that presented more concrete data, like the Watt-I-See gave more opportunity to user to act upon the information than systems that presented more abstract data (for example the SinaisFromFanal system). The WattsBurning, SINAIS Power Meter 1.0, 2.0 and the What-a-Watt system presented real-time information, this way they allowed for users to detect anomalies in their consumption in real time. Even though the SinaisFromFanal system also real time information, the presentation was too much abstract to allow anomaly detection. The WattsBurning in my mailbox and Watt-I-See systems did not alert for anomalies in consumption since the data presented was either not real time (WattsBurning in my mailbox), or an aggregation of production data (Watt-I-See). Apart from the WattsBurning in my mailbox no other system developed in this thesis allowed any type of personalization of the feedback. The Information Intent of the data presented in our systems is positioned in the middle to high part of the scale, the systems that presented electricity production data, power events or social comparison besides the consumption are positioned higher than the systems that only presented real time consumption plus aggregated metrics (e.g. SINAIS Power Meter 1.0). Neither one of the developed systems allowed to automate any task in the household. 


\subsection{Comparison}

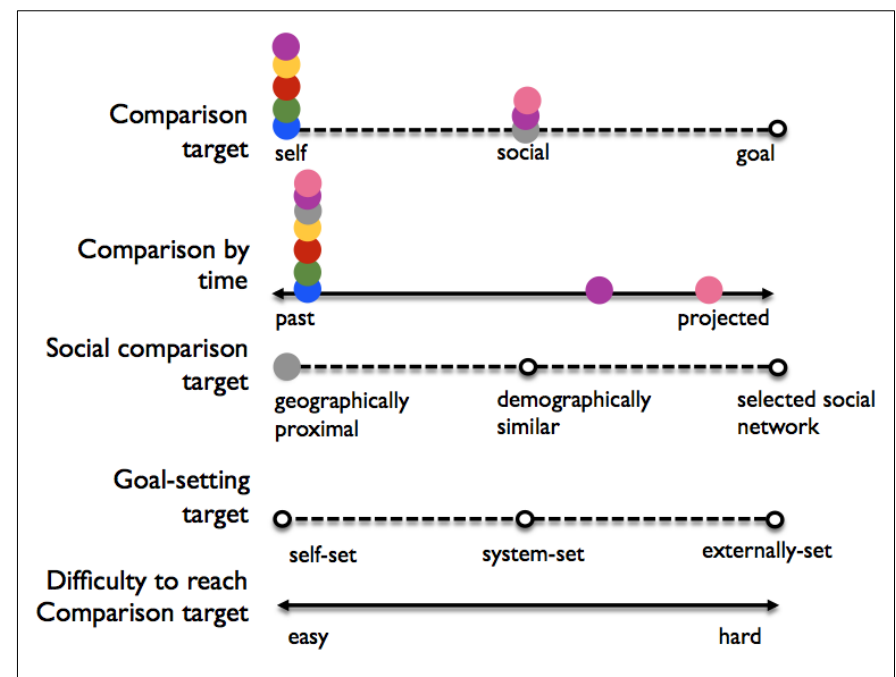

Figure 49: Comparison classification of the practical work of this thesis.

Considering how our system incorporated comparison in the feedback, all allowed comparison with self either being comparing electricity consumed (e.g. SINAIS Power Meter 2.0) or renewable energy consumed (Watt-I-See), furthermore the WattsBurning in my mailbox study allowed comparison between neighbours. Besides the What-a-Watt and Watt-I-See systems, which used the prediction of electricity production as a variable in the comparisons metrics, all the other systems used comparisons against past data. 


\subsection{Data representation}

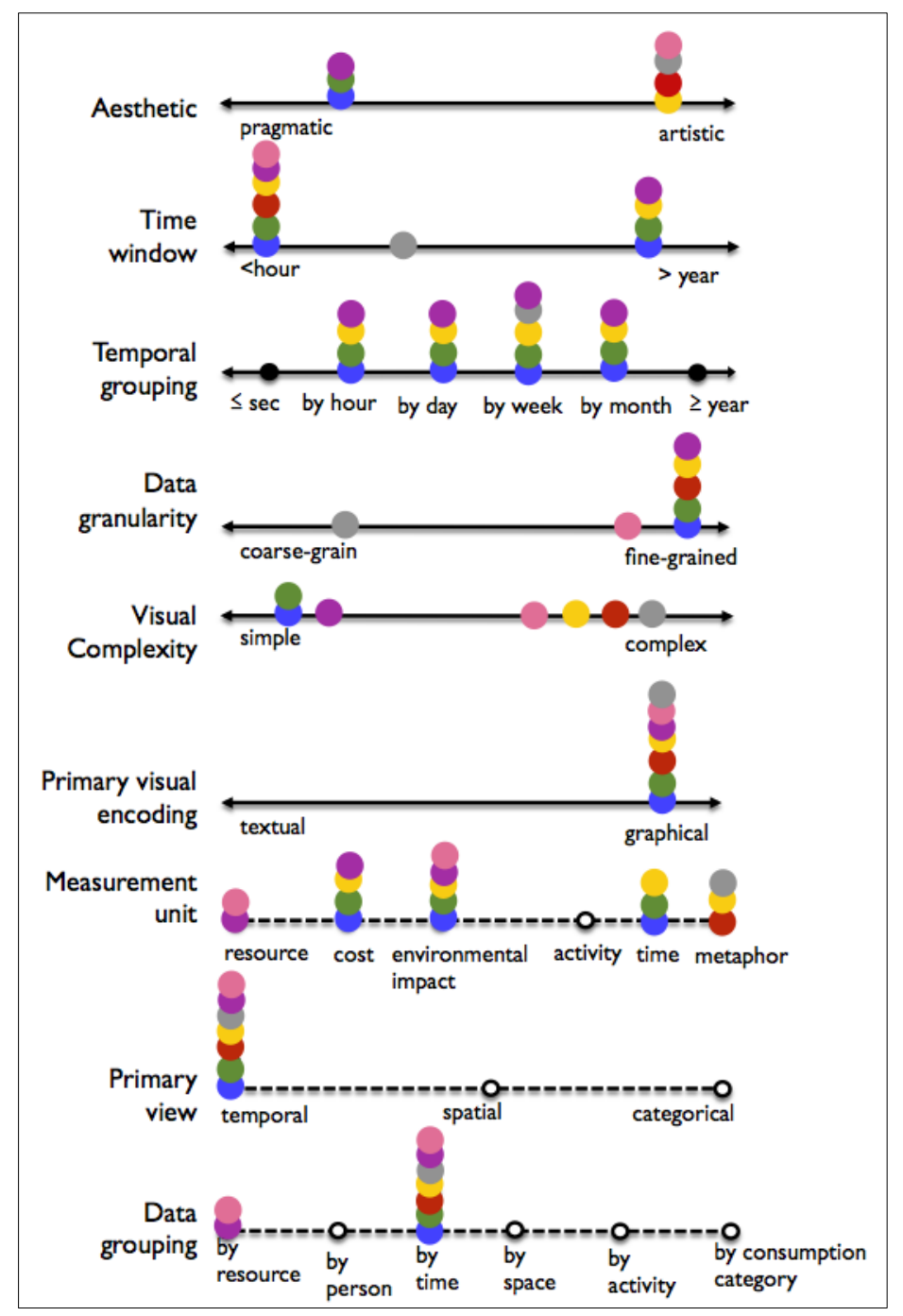

Figure 50: Data representation aspects of the practical work of this thesis.

The data representation is one of the central aspects on eco-feedback devices, this way our efforts are very varied in this scale (Figure 50). Regarding the Aesthetic sub-scale, the SINAIS Power Meter 1.0, 2.0 and Watt-I-See present the information in a more pragmatic manner, the other systems presented either animation or metaphors which made the feedback more artistic. The WattsBurning in my mailbox only presented data aggregated of one the week, however all the other systems allowed to consult data from different hours, days, months or even years, this observation is also true regarding how the data was grouped in the different screens of the interface of our systems. All of the systems that were used in the household presented real time data this way they are positioned in the right end of the data granularity scale, the Watt-I-See installation was only updated every 15 
minutes, therefore is positioned more towards the middle of the scale, lastly the WattsBurning in my mailbox study which grouped data by day of the week is positioned at the left end of the scale. The visual encoding of the information in another aspect in which our efforts can be positioned equally throughout the scale ranging from the concrete data in the SINAIS Power Meter 1.0 to the abstract representation of the WattsBurning in my mailbox. Our systems presented information using different measurements units, besides the cost $(€)$ impact in the environment $\left(\mathrm{CO}_{2}\right.$ emissions $)$ and time which were present our more "traditional" systems (e.g. SINAIS Power Meter 2.0), we also studied using the raw resources (electricity production data of the What-a-Watt and Watt-I-See installation) and metaphors (SinaisFromFanal and WattsBurning systems). All of our systems used a temporal variable (for example hour) to group the data, which was also the main representation for the presented data. However, the Watt-I-See and What-a-Watt studies also grouped information by resource, in those cases resources used to produce electricity

\subsection{Social Aspects}

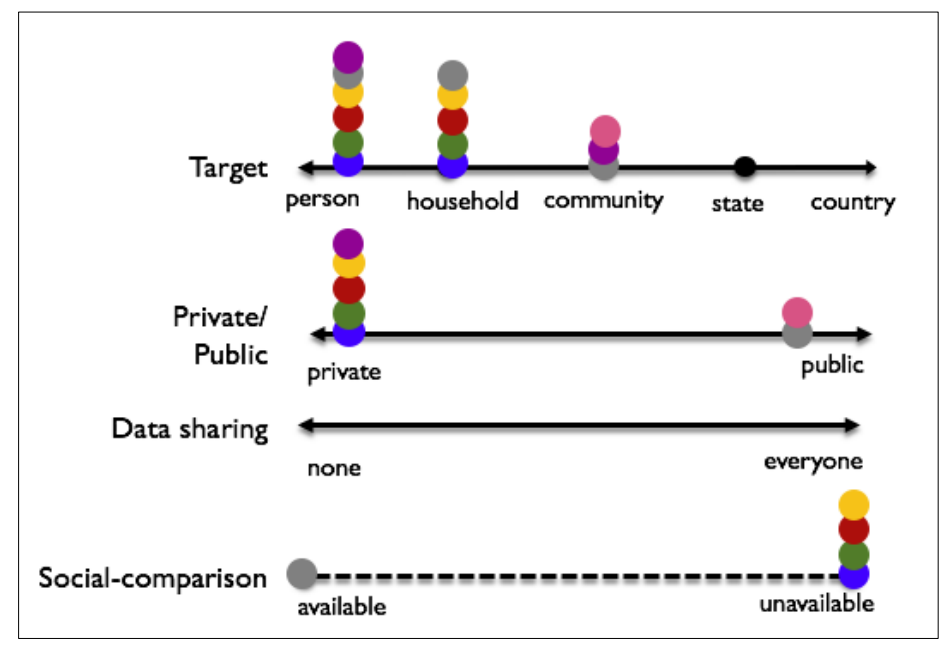

Figure 51: Social Aspects classification of the practical work of this thesis.

Most of our systems were not designed considering social variables, this way most of the systems only allowed self-comparisons, or household-wide comparison (e.g. SINAIS Power Meter 1.0 and 2.0), nonetheless the WattsBurning in my Mailbox system allowed for neighbours to compare their energy (social comparison) and the What-a-Watt and Watt-I-See presented island-wide production data. Two of our system were designed to be public (the Watt-I-See and the WattsBurning in my Mailbox) and the rest of were intended for domestic personal use. 


\subsection{Motivation/Persuasive techniques}

Regarding the Motivation/Persuasive techniques identified in the design space of ecofeedback, the WattsBurning What-a-Watt and Watt-I-See systems employed strategies such as likeability and emotional appeal while the 'WattsBurning on my mailbox' prototype used these two strategies in conjunction with Likeability, Competition and Social proof. The What-a-Watt and WattI-See also used the scarcity principle by presenting the resources used to produce electricity as something that is finite and valuable.

\subsection{Comparison Aspects}

Table 7: Comparison aspects of our prototypes.

\begin{tabular}{|c|c|c|c|}
\hline Prototype & Comparison variables & Statistics & Computation \\
\hline $\begin{array}{l}\text { SINAIS Power Meter } \\
1.0\end{array}$ & $\begin{array}{l}\text { Day, Week, Month, } \\
\text { Watt, kWh, } \mathrm{gCO}_{2} \text {, Euros }\end{array}$ & $\begin{array}{l}\text { Raw value, } \\
\text { Average }\end{array}$ & Yesterday, last week \\
\hline $\begin{array}{l}\text { SINAIS Power Meter } \\
2.0\end{array}$ & $\begin{array}{l}\text { Day, Week, Month, } \\
\text { Watt, kWh, } \mathrm{gCO}_{2} \text {, Euros }\end{array}$ & $\begin{array}{l}\text { Raw value, } \\
\text { Average }\end{array}$ & $\begin{array}{l}\text { Yesterday, last week, } \\
\text { last month }\end{array}$ \\
\hline SinaisFromFanal & Watt & Raw value & Yesterday, last week \\
\hline WattsBurning & $\begin{array}{l}\text { Day, Week, Month, } \\
\text { Watt, kWh, } \mathrm{gCO}_{2} \text {, Euros }\end{array}$ & $\begin{array}{l}\text { Raw value, } \\
\text { Average }\end{array}$ & $\begin{array}{l}\text { Yesterday, last week, } \\
\text { last month, days of the } \\
\text { week }\end{array}$ \\
\hline $\begin{array}{l}\text { WattsBurning on } \\
\text { my mailbox }\end{array}$ & Week & Average & Day of the week \\
\hline What-a-Watt & $\begin{array}{l}\text { Day, Week, Month, } \\
\text { Watt, kWh, } \mathrm{gCO}_{2} \text {, Euros }\end{array}$ & $\begin{array}{l}\text { Raw value, } \\
\text { Average }\end{array}$ & $\begin{array}{l}\text { Yesterday, last week, } \\
\text { last month, days of the } \\
\text { week }\end{array}$ \\
\hline
\end{tabular}




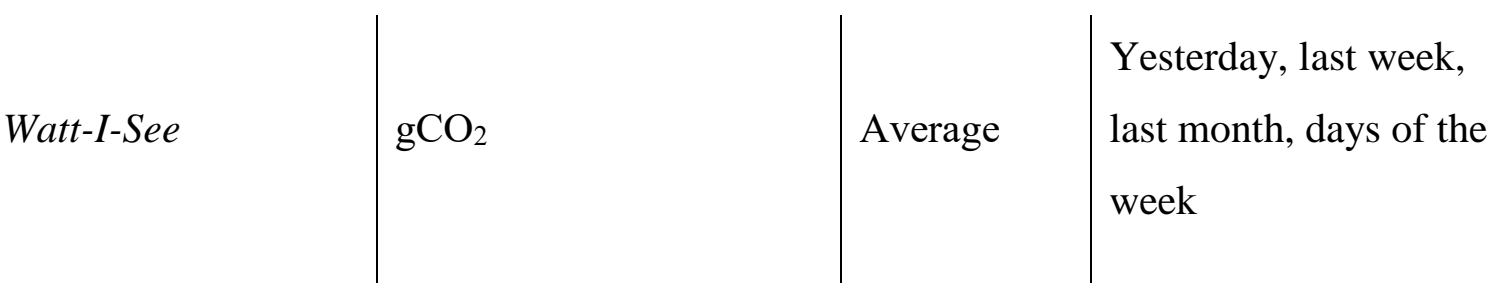

Our eco-feedback devices have use comparison widely in the presentation, the table above (Table 7), presents a summary of the computation aspects used in our systems. In general, we deployed comparisons using raw $\mathrm{kWh}$ or $\mathrm{CO}_{2}$, these variables were also aggregated and used in historical comparisons against passed days, weeks or months. For example, the power socket of the Watt-I-See system used a model which considered past daily, weekly and monthly emissions, the SINAIS Power Meter 2.0 used comparisons against past days, weeks and months 


\section{Conclusions}

In this chapter we present the main conclusions of this thesis. We already presented individual conclusions for each individual research study (sections 4.3.2.4, 4.3.3.6, 4.4.6, 4.5.8, 4.6.7, 4.7.10 and 4.8.10), but in this section we go back to the research questions presented in the introduction of this document and answer them based on our results.

\subsection{RQ1: Can we detect the novelty effect in eco-feedback systems deployed in the wild?}

We can answer to RQ1 plainly, the novelty effect was in fact found in the four long term studies performed during the period of this thesis. Although several participants from each study actually used the system more frequently, these were motivated individuals which do not represent the average population which lose the interest after roughly a month with the eco-feedback system.

\subsubsection{Implications}

The implications of RQ1 was itself verifying that the novelty exists in eco-feedback devices. What does this mean for the next steps of eco-feedback research? We argue that efforts should be focused on understanding why do certain individuals keep using the eco-feedback while others stop using these systems. In general, these systems are used during an exploratory phase when consumers explored their consumption, then they go to a second plan and only used when a change in the bill is noted. Perhaps, eco-feedback systems should be able to detect those changes in consumption and inform users before the abnormal bill gets charged so users can actually act upon it.

\subsection{RQ2: Is the novelty effect related to variables such as location, representation and information?}

We did not found evidence that the novelty effect significantly changed with the modifications we have implemented in the eco-feedback. The latter systems running in a tablet were used slighter longer than the first ones, and users reported liking the portability of the device, which allowed a 
more flexible usage. However, we did not find a meaningful difference. Nonetheless the changes introduced in our eco-feedback did in fact presented important findings that we discuss in latter sections of this chapter (6.4.2).

\subsubsection{Implications}

Although different design strategies can be used to first lure in users to the eco-feedback, the long term effect does not appear to differ. A possible path to contradict this observation is to keep updating the eco-feedback with new personal information so the eco-feedback is never repeated and keeps the novelty feeling.

However, we can argue that the portability of the device is something useful since it allows for consumption information to be consulted anywhere, and it also moves the feedback away from the mains at the house which are possibly not easily accessible and afar from the youngsters in the household.

\subsection{RQ3:Can the novelty effect be related to relapsing behaviour prior to the exposure of the eco-feedback?}

We found average reduction in consumption in our studies, however the savings were in generally small and different between participants. There were several families that were not able to save energy at all. With the constant changes in the households, like new equipment, changes in the equipment condition, new family members' or different seasons is quite hard to make sense of small perceptual changes in the consumption. These facts hinder our capacity to answer RQ3. However, in our studies we found a consistent observation, families which interacted more with the system were able to save energy. This observation is inline with Brynjarsdottir statement that self-tracking only works while the user self tracks (Brynjarsdottir et al., 2012).

\subsubsection{Implications}

Our studies did not find any evidence that the relapse effect would not happen. This is something eco-feedback researchers need to consider. Like we discussed in the se section 6.2.1, there is room for research into the individuals who actually used eco-feedback system every day without barely losing interest, and understand their motivations and goals. 
This way we believe that the biggest implication from RQ3 is that in fact there is the risk of consumers relapsing to previous behaviours of electricity consumption, like it was found in other fields.

\subsection{Other contributions}

\subsubsection{Positioning and feedback hardware}

Relating the feedback hardware with the pleasantness of the system is a straightforward conclusion, since different people have different preferences. However, our contribution here goes beyond this observation. The specificity of monitoring energy often restricts the placement of ecofeedback devices, furthermore the intrusiveness of these systems can also be a barrier for adoption. We observe this in our studies where in version 1.0 and 2.0 of the SINAIS Power Meter (section 4.3.2 and 4.3.3) families were worried about the safety of our installation, since it was connected to the mains of the house. This together with fact that the feedback in those versions of the system was given in a netBook placed up at approximately $1.6 \mathrm{~m}$ from the floor almost completely pushed the younger demographic away from the device. Youngsters were either unable to physical reach the device, or were discouraged to do so by their parents fearing an electric shock on the mains.

\subsubsection{Implications}

The design of eco-feedback devices should take into account not only the information needs of consumers. Consumers should feel confident about having these systems installed in their home. Although, we believe that the physical safety of the installations is improving to a point where there is really little to worry about, the amount of personal information smart meters can collect can surely be used to harm individuals if the data is obtained by a malicious third party. Therefore, our work also argues that these external needs of consumers should be considered when designing and operating eco-feedback devices.

\subsubsection{Using metaphors for displaying energy consumption}

In section 4.4 and 4.5, the SinaisFromFanal and WattsBurning systems displayed the consumption mapped in elements of the local forest. These two devices used metaphors to display the real-time household energy consumption. This approach was considered interesting and visually appealing by users since it used a scenery that users were familiar with. In fact, users were used in 
the decision of what elements to present (landscape, animals) in the eco-feedback. However, users did not reported any kind of emotional attachment to the system of moral obligation when it. We also observed (in SinaisFromFanal section 4.4) that metaphors or abstract representations for consumption should be used carefully in eco-feedback. Researchers often relay on these techniques, trying to avoid negative reinforcement. However, if those metaphors are not clear the results might be frustration and disinterest by users, or in a worst case, user getting wrong information from the system, altogether these outcomes can lead to individuals stop using the devices at all.

\subsubsection{Implications}

Our studies have disclosed that displaying metaphors or animation representing individual (or group) energy consumption can be effective in getting people's attention to the information itself. This way, we argue these techniques are more appropriate to eco-feedback devices where the first impression is the more important, or the often the only impression. For example, public displays or one-time interventions where the information has to be passed quickly and it has to compete with other sources for users' attention. However, the initial appeal of metaphors animation or abstract representation does not ensure a long term interest with the system, and the behavioural patterns found in RQ1 and RQ3

\subsubsection{Displaying the source of energy.}

In our last two studies (sections 4.7 and 4.8) we explored using the source of energy as a variable in the feedback. These two exploratory studies disclosed that individuals are genuinely interested in that information despite what prior knowledge they might have about the source of energy. We also found that in general individuals were not aware of the local infrastructure used to produce electricity, thus being also unfamiliar with the sources of energy. Our work argues, that if in a small island where this infrastructure is still very visible to residents, there are still misconceptions about the electricity grid, this problem is to be bigger in large metropolitan areas where the electricity is sourced from several different places. Nonetheless, we observed that individuals quickly understood the connection between the different variable that influence the energy (electricity) being produced locally. The two studies mentioned above also disclosed that even though individuals are interested, and even concerned about the source of their electricity (after the study), it is hard to introduce changes in energy consumption routines, especially if those changes are connected to unpredictable variable such as the availability of "green" energy. 


\subsubsection{Implications}

Our evaluation disclosed that users do in fact like the production information. This way the first implication of our work is the suggestion that this information should be added to eco-feedback devices as a complement to the individual consumption information. However, our contribution here goes further, as the electricity grid changes micro-generation will have an important role to play. This way we argue that understanding the effort and logic behind electricity production will crucial in the future of eco-feedback devices, either to inform consumers or to work together with automated agents that take decisions in the household. 


\section{References}

Abrahamse, W., Steg, L., Vlek, C., Rothengatter, T., 2007. The effect of tailored information, goal setting, and tailored feedback on household energy use, energy-related behaviors, and behavioral antecedents. J. Environ. Psychol. 27, 265-276. doi:10.1016/j.jenvp.2007.08.002

Alcott, B., 2010. Impact caps: why population, affluence and technology strategies should be abandoned. J. Clean. Prod., Growth, Recession or Degrowth for Sustainability and Equity? 18, 552-560. doi:10.1016/j.jclepro.2009.08.001

Alexakhin, R., Anspaugh, L., Balonov, M., Batandjieva, B., Besnus, F., Biesold, H., Bogdevich, I., Byron, D., Carr, Z., Deville-Cavelin, G., Ferris, I., Fesenko, S., Gentner, N., Golikov, V., Gora, A., Hendry, J., Hinton, T., Howard, B.J., Kashparov, V., Kirchner, G., LaGuardia, T., Linsley, G., Louvat, D., Moberg, L., Napier, B., Prister, B., Proskura, M., Reisenweaver, D., Schmieman, E., Shaw, G., Shestopalov, V., Smith, J., Strand, P., Tsaturov, Y., Vojtsekhovich, O., Woodhead, D., 2006. Environmental consequences of the Chernobyl accident and their remediation: Twenty years of experience. Report of the Chernobyl Forum Expert group "Environment." International Atomic Energy Agency, Vienna.

Antle, A.N., Droumeva, M., Ha, D., 2009. Hands on what?: comparing children's mouse-based and tangible-based interaction, in: Proceedings of the 8th International Conference on Interaction Design and Children. ACM, pp. 80-88.

Attari, S.Z., DeKay, M.L., Davidson, C.I., Bruine de Bruin, W., 2010. Public perceptions of energy consumption and savings. Proc. Natl. Acad. Sci. 107, 16054-16059. doi:10.1073/pnas.1001509107

Backlund, S., Gyllensvärd, M., Gustafsson, A., Ilstedt Hjelm, S., Mazé, R., Redström, J., 2006. Static! : The aesthetics of energy in everyday life. Presented at the WonderGround 2006, CEIADE Centro Editorial do IADE. 
Bakker, S., van den Hoven, E., Antle, A.N., 2011. MoSo Tangibles: Evaluating Embodied Learning, in: Proceedings of the Fifth International Conference on Tangible, Embedded, and Embodied Interaction, TEI '11. ACM, New York, NY, USA, pp. 85-92. doi:10.1145/1935701.1935720

Barreto, M., Karapanos, E., Nunes, N., 2011. Social Translucence as a Theoretical Framework for Sustainable HCI, in: Campos, P., Graham, N., Jorge, J., Nunes, N., Palanque, P., Winckler, M. (Eds.), Human-Computer Interaction - INTERACT 2011, Lecture Notes in Computer Science. Springer Berlin Heidelberg, pp. 195-203.

Barreto, M.L., Szóstek, A., Karapanos, E., Nunes, N.J., Pereira, L., Quintal, F., 2014. Understanding families' motivations for sustainable behaviors. Comput. Hum. Behav. 40, 6-15. doi:10.1016/j.chb.2014.07.042

Bénabou, R., Tirole, J., 2003. Intrinsic and Extrinsic Motivation. Rev. Econ. Stud. 70, 489-520. doi:10.1111/1467-937X.00253

Bird, J., Rogers, Y., 2010. The Pulse of Tidy Street: Measuring and Publicly Displaying Domestic Electricity Consumption, in: Workshop on Energy Awareness and Conservation through Pervasive Applications. Presented at the Pervasive, Helsinki.

Broms, L., Katzeff, C., B \aang, M., Nyblom, \AAsa, Hjelm, S.I., Ehrnberger, K., 2010. Coffee maker patterns and the design of energy feedback artefacts, in: Proceedings of the 8th ACM Conference on Designing Interactive Systems, DIS '10. ACM, New York, NY, USA, pp. 93 102. doi:10.1145/1858171.1858191

Brynjarsdottir, H., H\a akansson, M., Pierce, J., Baumer, E., DiSalvo, C., Sengers, P., 2012. Sustainably Unpersuaded: How Persuasion Narrows Our Vision of Sustainability, in: Proceedings of the SIGCHI Conference on Human Factors in Computing Systems, CHI '12. ACM, New York, NY, USA, pp. 947-956. doi:10.1145/2207676.2208539

Chamberlain, A., Crabtree, A., Rodden, T., Jones, M., Rogers, Y., 2012. Research in the Wild (RITW 2012) as part of ACM DIS 2012.

Chetty, M., Tran, D., Grinter, R.E., 2008. Getting to green: understanding resource consumption in the home, in: Proceedings of the 10th International Conference on Ubiquitous Computing, UbiComp '08. ACM, New York, NY, USA, pp. 242-251. doi:10.1145/1409635.1409668 
Cohn, G., Gupta, S., Froehlich, J., Larson, E., Patel, S.N., 2010. GasSense: appliance-level, singlepoint sensing of gas activity in the home, in: Proceedings of the 8th International Conference on Pervasive Computing, Pervasive'10. Springer-Verlag, Berlin, Heidelberg, pp. 265-282. doi:10.1007/978-3-642-12654-3_16

Colley, J.A., Bedwell, B., Crabtree, A., Rodden, T., 2013. Exploring Reactions to Widespread Energy Monitoring, in: Kotzé, P., Marsden, G., Lindgaard, G., Wesson, J., Winckler, M. (Eds.), Human-Computer Interaction - INTERACT 2013, Lecture Notes in Computer Science. Springer, Heidelberg, pp. 91-108.

Consolvo, S., Everitt, K., Smith, I., Landay, J.A., 2006. Design requirements for technologies that encourage physical activity, in: Proceedings of the SIGCHI Conference on Human Factors in Computing Systems, CHI '06. ACM, New York, NY, USA, pp. 457-466. doi:10.1145/1124772.1124840

Consolvo, S., McDonald, D.W., Landay, J.A., 2009. Theory-driven Design Strategies for Technologies That Support Behavior Change in Everyday Life, in: Proceedings of the SIGCHI Conference on Human Factors in Computing Systems, CHI '09. ACM, New York, NY, USA, pp. 405 414. doi:10.1145/1518701.1518766

Cosley, D., Lewenstein, J., Herman, A., Holloway, J., Baxter, J., Nomura, S., Boehner, K., Gay, G., 2008. ArtLinks: Fostering Social Awareness and Reflection in Museums, in: Proceedings of the SIGCHI Conference on Human Factors in Computing Systems, CHI '08. ACM, New York, NY, USA, pp. 403-412. doi:10.1145/1357054.1357121

Costanzo, M., Archer, D., Aronson, E., Pettigrew, T., 1986. Energy conservation behavior: The difficult path from information to action. Am. Psychol. 41, 521-528. doi:10.1037/0003066X.41.5.521

Darby, S., 2001. Making it Obvious: Designing Feedback into Energy Consumption, in: Bertoldi, D.P., Ricci, D.A., Almeida, P.A. de (Eds.), Energy Efficiency in Household Appliances and Lighting. Springer Berlin Heidelberg, pp. 685-696.

Demanding standards: Hydro One aims to leverage AMI via interoperability [WWW Document], 2007. URL http://www.elp.com/articles/2007/10/demanding-standards-hydro-one-aims-toleverage-ami-via-interoperability.html (accessed 1.9.15). 
Dow, S., MacIntyre, B., 2007. New Media Collaboration through Wizard-of Oz Simulations, in: Computer-Human Interaction.

Dunlap, R.E., Van Liere, K.D., Mertig, A.G., Jones, R.E., 2000. New trends in measuring environmental attitudes: measuring endorsement of the new ecological paradigm: a revised NEP scale. J. Soc. Issues 56, 425-442.

E-Energy, Federal Ministry for the Environment, Nature Conservation, Building and Nuclear Safety [WWW Document], 2007. URL http://www.elp.com/articles/2007/10/demanding-standardshydro-one-aims-to-leverage-ami-via-interoperability.html (accessed 1.9.15).

Erickson, T., Li, M., Kim, Y., Deshpande, A., Sahu, S., Chao, T., Sukaviriya, P., Naphade, M., 2013. The dubuque electricity portal: evaluation of a city-scale residential electricity consumption feedback system, in: Proceedings of the SIGCHI Conference on Human Factors in Computing Systems, CHI '13. ACM, New York, NY, USA, pp. 1203-1212. doi:10.1145/2470654.2466155

Ernevi, A., Jacobs, M., Mazé, R., Müller, C., Redström, J., Worbin, L., 2005. The Energy Curtain: Energy Awareness, in: Redström, J., Redström, M., Mazé, R. (Eds.), IT+Textiles. (2010 reprint) Borås, Sweden: Centre for Textile Research. (2005 first edition) Helsinki: IT Press/Edita, pp. 91-96.

Filipe Quintal, L.P., 2012. A long-term study of energy eco-feedback using non-intrusive load monitoring. Persuas. Technol. 49.

Filonik, D., Medland, R., Foth, M., Rittenbruch, M., 2013. A Customisable Dashboard Display for Environmental Performance Visualisations, in: Berkovsky, S., Freyne, J. (Eds.), Persuasive Technology, Lecture Notes in Computer Science. Springer Berlin Heidelberg, pp. 51-62.

Fischer, C., 2008. Feedback on household electricity consumption: a tool for saving energy? Energy Effic. 1, 79-104. doi:10.1007/s12053-008-9009-7

Fischer, J.E., Ramchurn, S.D., Osborne, M.A., Parson, O., Huynh, T.D., Alam, M., Pantidi, N., Moran, S., Bachour, K., Reece, S., Costanza, E., Rodden, T., Jennings, N.R., 2013. Recommending Energy Tariffs and Load Shifting Based on Smart Household Usage Profiling. Presented at the International Conference on Intelligent User Interfaces, pp. 383-394. 
Fitzpatrick, G., Smith, G., 2009. Technology-Enabled Feedback on Domestic Energy Consumption: Articulating a Set of Design Concerns. IEEE Pervasive Comput. 8, 37-44. doi:10.1109/MPRV.2009.17

Fogg, B., 2009. A Behavior Model for Persuasive Design, in: Proceedings of the 4th International Conference on Persuasive Technology, Persuasive '09. ACM, New York, NY, USA, pp. 40:140:7. doi:10.1145/1541948.1541999

Foster, D., Lawson, S., Blythe, M., Cairns, P., 2010. Wattsup?: motivating reductions in domestic energy consumption using social networks, in: Proceedings of the 6th Nordic Conference on Human-Computer Interaction: Extending Boundaries, NordiCHI '10. ACM, New York, NY, USA, pp. 178-187. doi:10.1145/1868914.1868938

Froehlich, J., Dillahunt, T., Klasnja, P., Mankoff, J., Consolvo, S., Harrison, B., Landay, J.A., 2009a. UbiGreen: investigating a mobile tool for tracking and supporting green transportation habits, in: Proceedings of the SIGCHI Conference on Human Factors in Computing Systems, CHI '09. ACM, New York, NY, USA, pp. 1043-1052. doi:10.1145/1518701.1518861

Froehlich, J., Dillahunt, T., Klasnja, P., Mankoff, J., Consolvo, S., Harrison, B., Landay, J.A., $2009 b$. UbiGreen: investigating a mobile tool for tracking and supporting green transportation habits, in: Proceedings of the SIGCHI Conference on Human Factors in Computing Systems, CHI '09. ACM, New York, NY, USA, pp. 1043-1052. doi:10.1145/1518701.1518861

Froehlich, J.E., 2012. Sensing and Feedback of Everyday Activities to Promote Environmental Behaviors. UNIVERSITY OF WASHINGTON.

Froehlich, J.E., Larson, E., Campbell, T., Haggerty, C., Fogarty, J., Patel, S.N., 2009. HydroSense: infrastructure-mediated single-point sensing of whole-home water activity, in: Proceedings of the 11th International Conference on Ubiquitous Computing, Ubicomp '09. ACM, New York, NY, USA, pp. 235-244. doi:10.1145/1620545.1620581

Froehlich, J., Findlater, L., Landay, J., 2010. The design of eco-feedback technology, in: Proceedings of the SIGCHI Conference on Human Factors in Computing Systems, CHI '10. ACM, New York, NY, USA, pp. 1999-2008. doi:10.1145/1753326.1753629 
Froehlich, J., Findlater, L., Ostergren, M., Ramanathan, S., Peterson, J., Wragg, I., Larson, E., Fu, F., Bai, M., Patel, S., Landay, J.A., 2012. The design and evaluation of prototype eco-feedback displays for fixture-level water usage data, in: Proceedings of the SIGCHI Conference on Human Factors in Computing Systems, CHI '12. ACM, New York, NY, USA, pp. 2367-2376. doi:10.1145/2207676.2208397

Gamberini, L., Spagnolli, A., Corradi, N., Jacucci, G., Tusa, G., Mikkola, T., Zamboni, L., Hoggan, E., 2012a. Tailoring Feedback to Users' Actions in a Persuasive Game for Household Electricity Conservation, in: Bang, M., Ragnemalm, E.L. (Eds.), Persuasive Technology. Design for Health and Safety, Lecture Notes in Computer Science. Springer Berlin Heidelberg, pp. 100-111.

Gamberini, L., Spagnolli, A., Corradi, N., Jacucci, G., Tusa, G., Mikkola, T., Zamboni, L., Hoggan, E.E., 2012b. Tailoring Feedback to Users’'Actions in a Persuasive Game for Household Electricity Conservation. Presented at the PERSUASIVE, pp. 100-111.

Gupta, S., Reynolds, M.S., Patel, S.N., 2010. ElectriSense: single-point sensing using EMI for electrical event detection and classification in the home, in: Proceedings of the 12th ACM International Conference on Ubiquitous Computing, Ubicomp '10. ACM, New York, NY, USA, pp. 139-148. doi:10.1145/1864349.1864375

Gustafsson, A., Gyllenswärd, M., 2005. The power-aware cord: energy awareness through ambient information display, in: CHI '05 Extended Abstracts on Human Factors in Computing Systems, CHI EA '05. ACM, New York, NY, USA, pp. 1423-1426. doi:10.1145/1056808.1056932

Hargreaves, T., Nye, M., Burgess, J., 2010. Making energy visible: A qualitative field study of how householders interact with feedback from smart energy monitors. Energy Policy 38, 61116119.

Hart, G.W., 1992. Nonintrusive appliance load monitoring. Proc. IEEE 80, 1870-1891. doi:10.1109/5.192069

Hay, S., Rice, A., 2009. The case for apportionment, in: Proceedings of the First ACM Workshop on Embedded Sensing Systems for Energy-Efficiency in Buildings, BuildSys '09. ACM, New York, NY, USA, pp. 13-18. doi:10.1145/1810279.1810283 
He, H.A., Greenberg, S., Huang, E.M., 2010. One size does not fit all: applying the transtheoretical model to energy feedback technology design, in: Proceedings of the SIGCHI Conference on Human Factors in Computing Systems, CHI '10. ACM, New York, NY, USA, pp. 927-936. doi:10.1145/1753326.1753464

Hledik, R., 2009. How Green Is the Smart Grid? Electr. J. 22, 29-41. doi:10.1016/j.tej.2009.03.001

Hockenbury, D.H., Hockenbury, S.E., 2012. Psychology. Worth Publishers.

Holmes, T.G., 2007. Eco-visualization: combining art and technology to reduce energy consumption, in: Proceedings of the 6th ACM SIGCHI Conference on Creativity \&amp; Cognition, C\&amp;C ’07. ACM, New York, NY, USA, pp. 153-162. doi:10.1145/1254960.1254982

Horn, M.S., Crouser, R.J., Bers, M.U., 2012. Tangible interaction and learning: the case for a hybrid approach. Pers. Ubiquitous Comput. 16, 379-389.

Inc, E.F. for N.S., 1972. Bulletin of the Atomic Scientists. Educational Foundation for Nuclear Science, Inc.

International Energy Outlook 2013, 2013. . United States Energy Information Administration.

Jakobi, T., Schwartz, T., 2012. Putting the user in charge: End user development for eco-feedback technologies, in: Sustainable Internet and ICT for Sustainability (SustainIT), 2012. Presented at the Sustainable Internet and ICT for Sustainability (SustainIT), 2012, pp. 1-4.

James Pierce, C.F., 2010. Some consideration on the (in)effectiveness of residential energy feedback systems. 244-247. doi:10.1145/1858171.1858215

Johnson, D.L., Ambrose, S.H., Bassett, T.J., Bowen, M.L., Crummey, D.E., Isaacson, J.S., Johnson, D.N., Lamb, P., Saul, M., Winter-Nelson, A.E., 1997. Meanings of Environmental Terms. J. Environ. Qual. 26. doi:10.2134/jeq1997.00472425002600030002x

Jönsson, L., Broms, L., Katzeff, C., 2010. Watt-Lite: energy statistics made tangible, in: Proceedings of the 8th ACM Conference on Designing Interactive Systems, DIS '10. ACM, New York, NY, USA, pp. 240-243. doi:10.1145/1858171.1858214 
Kim, Y., Schmid, T., Charbiwala, Z.M., Srivastava, M.B., 2009. ViridiScope: Design and Implementation of a Fine Grained Power Monitoring System for Homes, in: Proc. Ubicomp09, $245-254$.

Kjeldskov, J., Skov, M.B., Paay, J., Lund, D., Madsen, T., Nielsen, M., 2015. Eco-Forecasting for Domestic Electricity Use, in: Proceedings of the 33rd Annual ACM Conference on Human Factors in Computing Systems, CHI '15. ACM, New York, NY, USA, pp. 1985-1988. doi: $10.1145 / 2702123.2702318$

Kjeldskov, J., Skov, M.B., Paay, J., Pathmanathan, R., 2012a. Using Mobile Phones to Support Sustainability: A Field Study of Residential Electricity Consumption, in: Proceedings of the SIGCHI Conference on Human Factors in Computing Systems, CHI '12. ACM, New York, NY, USA, pp. 2347-2356. doi:10.1145/2207676.2208395

Kjeldskov, J., Skov, M.B., Paay, J., Pathmanathan, R., 2012b. Using Mobile Phones to Support Sustainability: A Field Study of Residential Electricity Consumption, in: Proceedings of the SIGCHI Conference on Human Factors in Computing Systems, CHI '12. ACM, New York, NY, USA, pp. 2347-2356. doi:10.1145/2207676.2208395

Kohlenberg, R., Phillips, T., Proctor, W., 1976. A behavioral analysis of peaking in residential electrical-energy consumers. J. Appl. Behav. Anal. 9, 13-18. doi:10.1901/jaba.1976.9-13

Mankoff, J., Fussell, S.R., Dillahunt, T., Glaves, R., Grevet, C., Johnson, M., Matthews, D., Matthews, H.S., McGuire, R., Thompson, R., Shick, A., Setlock, L., 2010. StepGreen.org: Increasing Energy Saving Behaviors via Social Networks, in: Fourth International AAAI Conference on Weblogs and Social Media. Presented at the Fourth International AAAI Conference on Weblogs and Social Media.

McCalley, L.., Midden, C.J.., 2002. Energy conservation through product-integrated feedback: The roles of goal-setting and social orientation. J. Econ. Psychol. 23, 589-603. doi:10.1016/S01674870(02)00119-8

Miller, W., Buys, L., 2010. Householder experiences with resource monitoring technology in sustainable homes, in: Proceedings of the 22nd Conference of the Computer-Human Interaction Special Interest Group of Australia on Computer-Human Interaction, OZCHI '10. ACM, New York, NY, USA, pp. 228-231. doi:10.1145/1952222.1952271 
Mills, E., 2002. Review and comparison of web-and disk-based tools for residential energy analysis. Lawrence Berkeley Natl. Lab.

Moere, A.V., Tomitsch, M., Hoinkis, M., Trefz, E., Johansen, S., Jones, A., 2011. Comparative feedback in the street: exposing residential energy consumption on house fa\&\#231;ades, in: Proceedings of the 13th IFIP TC 13 International Conference on Human-Computer Interaction - Volume Part I, INTERACT’11. Springer-Verlag, Berlin, Heidelberg, pp. 470-488.

Motegi, N., Piette, M.A., Kinney, S., Herter, K., 2003. Web-Based Energy Information Systems for Energy Management and Demand Response in Commercial Buildings [WWW Document].

Nunes, N.J., Pereira, L., Quintal, F., Berges, M., 2011. Deploying and evaluating the effectiveness of energy eco-feedback through a low-cost NILM solution, in: Proceedings of the 6th International Conference on Persuasive Technology. pp. 2-5.

Odom, W., Pierce, J., Roedl, D., 2008. Social Incentive \& Eco-Visualization Displays: Toward Persuading Greater Change in Dormitory Communities, in: Workshop Proc. Of OZCHI.

O’Neill, S., Nicholson-Cole, S., 2009. “Fear Won't Do It" Promoting Positive Engagement With Climate Change Through Visual and Iconic Representations. Sci. Commun. 30, 355-379. doi:10.1177/1075547008329201

Patel, S.N., Robertson, T., Kientz, J.A., Reynolds, M.S., Abowd, G.D., 2007. At the flick of a switch: detecting and classifying unique electrical events on the residential power line, in: Proceedings of the 9th International Conference on Ubiquitous Computing, UbiComp '07. Springer-Verlag, Berlin, Heidelberg, pp. 271-288.

Pereira, L., Quintal, F., Barreto, M., Nunes, N.J., 2013. Understanding the Limitations of Ecofeedback: A One-Year Long-Term Study, in: Holzinger, A., Pasi, G. (Eds.), Human-Computer Interaction and Knowledge Discovery in Complex, Unstructured, Big Data, Lecture Notes in Computer Science. Springer Berlin Heidelberg, pp. 237-255.

Pereira, L., Quintal, F., Nunes, N., Bergés, M., 2012. The Design of a Hardware-software Platform for Long-term Energy Eco-feedback Research, in: Proceedings of the 4th ACM SIGCHI Symposium on Engineering Interactive Computing Systems, EICS '12. ACM, New York, NY, USA, pp. 221-230. doi:10.1145/2305484.2305521 
Peschiera, G., Taylor, J.E., Siegel, J.A., 2010. Response-relapse patterns of building occupant electricity consumption following exposure to personal, contextualized and occupant peer network utilization data. Energy Build. 42, 1329-1336. doi:10.1016/j.enbuild.2010.03.001

Petersen, J.E., Shunturov, V., Janda, K., Platt, G., Weinberger, K., 2007. Dormitory residents reduce electricity consumption when exposed to real-time visual feedback and incentives. Int. J. Sustain. High. Educ. 8, 16-33. doi:10.1108/14676370710717562

Petersen, M.G., Iversen, O.S., Krogh, P.G., Ludvigsen, M., 2004. Aesthetic Interaction: A Pragmatist's Aesthetics of Interactive Systems, in: Proceedings of the 5th Conference on Designing Interactive Systems: Processes, Practices, Methods, and Techniques, DIS '04. ACM, New York, NY, USA, pp. 269-276. doi:10.1145/1013115.1013153

Petkov, P., Goswami, S., Köbler, F., Krcmar, H., 2012. Personalised eco-feedback as a design technique for motivating energy saving behaviour at home, in: Proceedings of the 7 th Nordic Conference on Human-Computer Interaction: Making Sense Through Design, NordiCHI '12. ACM, New York, NY, USA, pp. 587-596. doi:10.1145/2399016.2399106

Petkov, P., Köbler, F., Foth, M., Krcmar, H., 2011. Motivating domestic energy conservation through comparative, community-based feedback in mobile and social media, in: Proceedings of the 5th International Conference on Communities and Technologies, C\&\#38;T '11. ACM, New York, NY, USA, pp. 21-30. doi:10.1145/2103354.2103358

Petty, R.E., Cacioppo, J.T., Strathman, A.J., Priester, J.R., 2005. To think or not to think. Persuas. Psychol. Insights Perspect. 81-116.

Pierce, J., Odom, W., Blevis, E., 2008. Energy aware dwelling: a critical survey of interaction design for eco-visualizations, in: Proceedings of the 20th Australasian Conference on ComputerHuman Interaction: Designing for Habitus and Habitat, OZCHI '08. ACM, New York, NY, USA, pp. 1-8. doi:10.1145/1517744.1517746

Pierce, J., Paulos, E., 2012a. Beyond energy monitors: interaction, energy, and emerging energy systems, in: Proceedings of the SIGCHI Conference on Human Factors in Computing Systems, CHI '12. ACM, New York, NY, USA, pp. 665-674. doi:10.1145/2207676.2207771 
Pierce, J., Paulos, E., 2012b. The local energy indicator: designing for wind and solar energy systems in the home, in: Proceedings of the Designing Interactive Systems Conference, DIS '12. ACM, New York, NY, USA, pp. 631-634. doi:10.1145/2317956.2318050

Pierce, J., Paulos, E., 2010. Materializing energy, in: Proceedings of the 8th ACM Conference on Designing Interactive Systems, DIS '10. ACM, New York, NY, USA, pp. 113-122. doi:10.1145/1858171.1858193

Quintal, F., Pereira, L., Nunes, N., Nisi, V., Barreto, M., 2013. WATTSBurning: Design and Evaluation of an Innovative Eco-Feedback System, in: Kotzé, P., Marsden, G., Lindgaard, G., Wesson, J., Winckler, M. (Eds.), Human-Computer Interaction - INTERACT 2013, Lecture Notes in Computer Science. Springer Berlin Heidelberg, pp. 453-470.

Reading the clouds, 2008. . Nuage Vert.

Read, J., Fine, K., 2005. Using survey methods for design and evaluation in child computer interaction, in: Workshop on Child Computer Interaction: Methodological Research at Interact.

Riche, Y., Dodge, J., Metoyer, R.A., 2010. Studying always-on electricity feedback in the home, in: Proceedings of the SIGCHI Conference on Human Factors in Computing Systems, CHI '10. ACM, New York, NY, USA, pp. 1995-1998. doi:10.1145/1753326.1753628

Rodden, T.A., Fischer, J.E., Pantidi, N., Bachour, K., Moran, S., 2013. At home with agents: exploring attitudes towards future smart energy infrastructures, in: Proceedings of the SIGCHI Conference on Human Factors in Computing Systems, CHI '13. ACM, New York, NY, USA, pp. 1173-1182. doi:10.1145/2470654.2466152

Rodgers, J., Bartram, L., 2011. Exploring Ambient and Artistic Visualization for Residential Energy Use Feedback. IEEE Trans. Vis. Comput. Graph. 17, 2489-2497. doi:10.1109/TVCG.2011.196

Schipper, L., Bartlett, S., Hawk, D., Vine, E., 1989. Linking Life-Styles and Energy Use: A Matter of Time? Annu. Rev. Energy 14, 273-320. doi:10.1146/annurev.eg.14.110189.001421

Scott, M., Barreto, M., Quintal, F., Oakley, I., 2011. Understanding Goal Setting Behavior in the Context of Energy Consumption Reduction, in: Proceedings of the 13th IFIP TC 13 
International Conference on Human-Computer Interaction - Volume Part I, INTERACT'11. Springer-Verlag, Berlin, Heidelberg, pp. 129-143.

Simm, W., Ferrario, M.A., Friday, A., Newman, P., Forshaw, S., Hazas, M., Dix, A., 2015. Tiree Energy Pulse: Exploring Renewable Energy Forecasts on the Edge of the Grid, in: Proceedings of the 33rd Annual ACM Conference on Human Factors in Computing Systems, CHI '15. ACM, New York, NY, USA, pp. 1965-1974. doi:10.1145/2702123.2702285

Spagnolli, A., Corradi, N., Gamberini, L., Hoggan, E., Jacucci, G., Katzeff, C., Broms, L., Jonsson, L., 2011. Eco-Feedback on the Go: Motivating Energy Awareness. Computer 44, 38-45. doi:10.1109/MC.2011.125

Spence, A., Pidgeon, N., 2010. Framing and communicating climate change: The effects of distance and outcome frame manipulations. Glob. Environ. Change, 20th Anniversary Special Issue 20, 656-667. doi:10.1016/j.gloenvcha.2010.07.002

Spence, A., Poortinga, W., Pidgeon, N., 2012. The Psychological Distance of Climate Change. Risk Anal. 32, 957-972. doi:10.1111/j.1539-6924.2011.01695.x

Strengers, Y.A.A., 2011. Designing eco-feedback systems for everyday life, in: Proceedings of the SIGCHI Conference on Human Factors in Computing Systems, CHI '11. ACM, New York, NY, USA, pp. 2135-2144. doi:10.1145/1978942.1979252

Tilman, D., Lehman, C., 2001. Human-caused environmental change: Impacts on plant diversity and evolution. Proc. Natl. Acad. Sci. 98, 5433-5440. doi:10.1073/pnas.091093198

Ueno, T., Inada, R., Saeki, O., Tsuji, K., 2005. Effectiveness of displaying energy consumption data in residential houses. Analysis on how the residents respond. Proc. Eur. Counc. Energy-Effic. Econ. Pap. 6, 19.

Wallenborn, G., Orsini, M., Vanhaverbeke, J., 2011. Household appropriation of electricity monitors. Int. J. Consum. Stud. 35, 146-152. doi:10.1111/j.1470-6431.2010.00985.x

Weiss, M., Mattern, F., Graml, T., Staake, T., Fleisch, E., 2009. Handy feedback: connecting smart meters with mobile phones, in: Proceedings of the 8th International Conference on Mobile and Ubiquitous Multimedia, MUM '09. ACM, New York, NY, USA, pp. 15:1-15:4. doi: $10.1145 / 1658550.1658565$ 
Woodruff, A., Hasbrouck, J., Augustin, S., 2008. A bright green perspective on sustainable choices, in: Proceedings of the SIGCHI Conference on Human Factors in Computing Systems, CHI '08. ACM, New York, NY, USA, pp. 313-322. doi:10.1145/1357054.1357109

Wright, P.M., Kacmar, K.M., 1994. Goal Specificity as a Determinant of Goal Commitment and Goal Change. Organ. Behav. Hum. Decis. Process. 59, 242-260. doi:10.1006/obhd.1994.1059

Yang, R., Newman, M.W., Forlizzi, J., 2014. Making Sustainability Sustainable: Challenges in the Design of Eco-interaction Technologies, in: Proceedings of the SIGCHI Conference on Human Factors in Computing Systems, CHI '14. ACM, New York, NY, USA, pp. 823-832. doi:10.1145/2556288.2557380

Yun, T.-J., 2009. Investigating the impact of a minimalist in-home energy consumption display, in: CHI '09 Extended Abstracts on Human Factors in Computing Systems, CHI EA '09. ACM, New York, NY, USA, pp. 4417-4422. doi:10.1145/1520340.1520676

Zeifman, M., Roth, K., 2011. Nonintrusive appliance load monitoring: Review and outlook. IEEE Trans. Consum. Electron. 57, 76-84. doi:10.1109/TCE.2011.5735484

Zhang, Z., Shrubsole, P., Janse, M., 2010. Learning Environmental Factors Through Playful Interaction, in: Proceedings of the 9th International Conference on Interaction Design and Children, IDC '10. ACM, New York, NY, USA, pp. 166-173. doi:10.1145/1810543.1810562 
References 


\section{Appendices}

\subsection{Selection survey Sinais Power Meter 1.0}

Questionários_Energy Meter Study Phase I

Data № Apartamento

1. Esta é a sua primeira casa?

Sim Não

2. A casa é alugada?

Sim Não

3. A quanto tempo é que vive nesta casa?

4. Quantas pessoas vivem na casa?

adultos crianças

5. Idade dos adultos e crianças

Ex: Mãe/ 29 anos

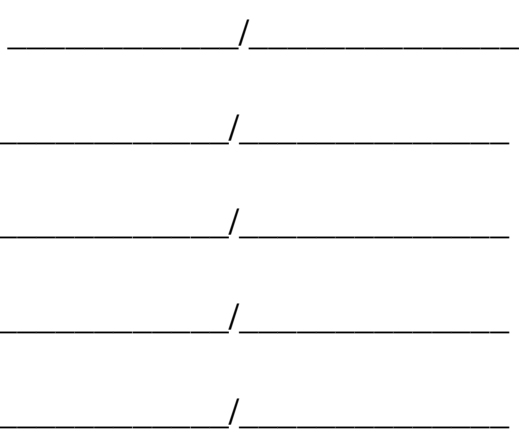


6. Profissões dos pais/adultos que vivem na casa:

Ex: Mãe/ Professora Primária

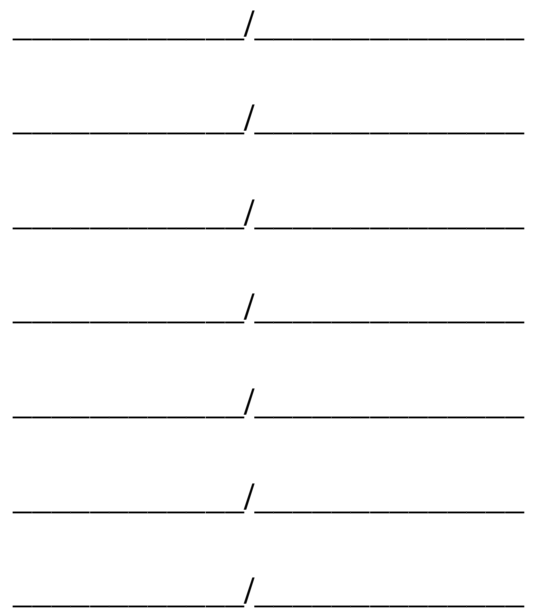

7. Nível de escolaridades das pessoas que vivem na casa:

Ex: Mãe/ Ensino Superior

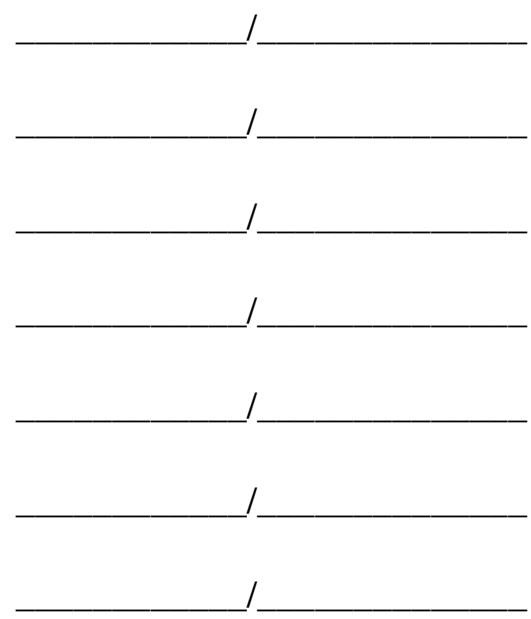

8. Quantos computadores têm em casa?

Fixos Portáteis

9. Tem internet em casa?

Sim Não

10. Tem alguma consola de jogos (playstation, wii, Xbox etc.)?

Sim Não

11. Tem pessoas na casa a usar websites/redes sociais?

Sim Quantas pessoas? Que redes sociais?

Não 
12. Sabe quanto pagou de electricidade e água no mês passado?

Electricidade euros Água euros

\section{Como se considera em termos de:}

A. Poupança/uso de energia/electricidade (escala de 1 a 5)

(Gasto muito pouco) $1 \ldots \ldots .2 \ldots \ldots 3 \ldots \ldots 4 \ldots \ldots 5$ (Gasto para além do necessário)

B. Separação do lixo/reciclagem

(Não faço a separação do lixo) $1 \ldots \ldots . .2 \ldots \ldots 3 \ldots \ldots . \ldots \ldots 5$ (Separo sempre o lixo)

C. Uso de transportes públicos

$$
\text { (Não uso ) } 1 \ldots \ldots . . \ldots \ldots 3 \ldots \ldots 4 \ldots \ldots 5 \text { (Uso todos os dias) }
$$

D. Poupança/uso de água

(Gasto muito pouco) $\quad 1 \ldots \ldots . \ldots \ldots 3 \ldots \ldots 4 \ldots \ldots 5$ (Gasto para além do necessário)

14. Estaria disponível para participar num estudo científico e anónimo que implica a instalação de um medidor de energia na sua casa para recolher informação durante vários meses?

$\operatorname{Sim}$

Contacto:
Não

Talvez

Outras informações/Notas 


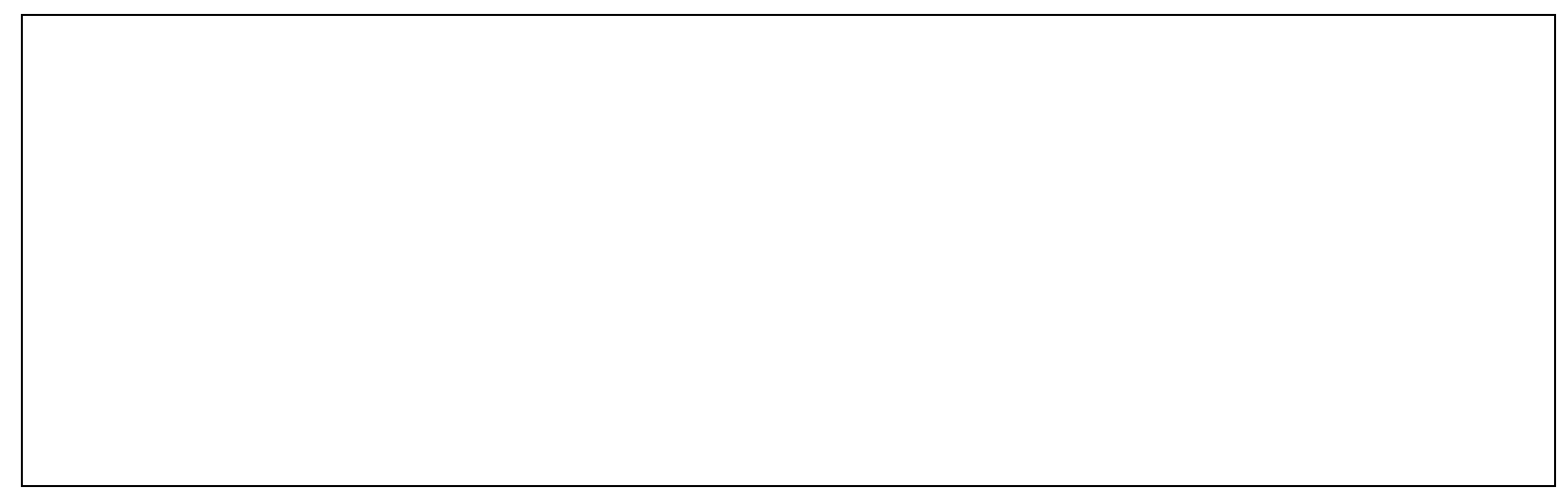




\subsection{Participants Survey Sinais Power Meter 1.0}

A. Por favor preencha os seguintes dados demográficos:

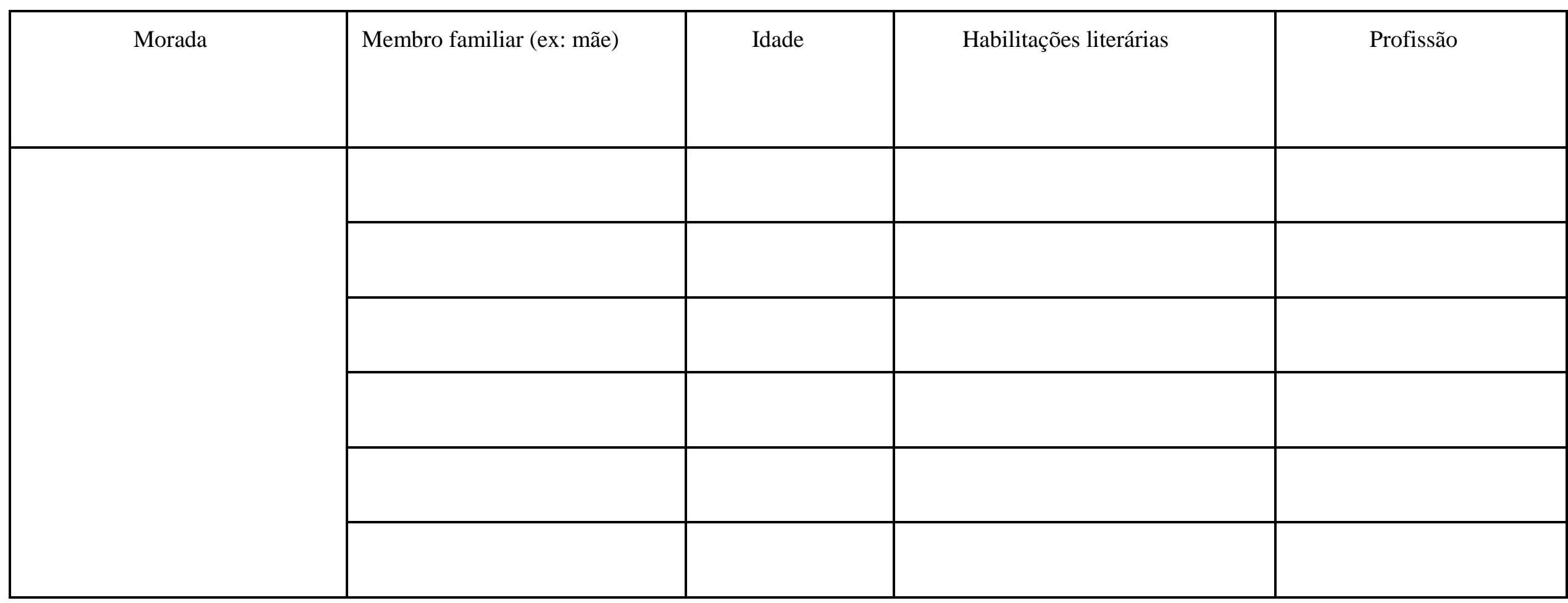

B. Poderia indicar o valor da sua conta de eletricidade dos últimos três meses?

Fevereiro $=$ Março $=$ Abril $=$

C. Por favor responda à seguinte questão sobre aquecimento global: 
1. Na sua opinião, em que medida acha que o aquecimento global é grave?

$\begin{array}{lllllll}\text { Nada grave } & 1 & 2 & 3 & 4 & 5 & \text { Muito grave }\end{array}$

2. Eu acredito que reduzir o consumo de energia terá um impacto positivo no ambiente
Discordo totalmente
1
2
3
4
5
Concordo totalmente

3. Eu acho que o meu consumo de energia é algo que...
Não preciso de preocupar-me
12

3

4
5 Preciso de me preocupar

4. A ligação entre aquecimento global e o consumo de energia é fraca ou inexistente
Discordo totalmente
23
45
Concordo totalmente

5. Eu preocupo-me com os possíveis efeitos do aquecimento global
Não estou preocupado(a)
12
3
4
5 Extremamente preocupado(a)

C. Quais as razões que o levariam a assumir um comportamento sustentável? 


\begin{tabular}{|c|c|c|c|c|c|}
\hline & De forma alguma & & & & Verdadeiramente \\
\hline Porque sinto que tenho que o fazer & 1 & 2 & 3 & 4 & 5 \\
\hline Porque é interessante e algo agradável de fazer & 1 & 2 & 3 & 4 & 5 \\
\hline Porque sinto que devo faze-lo ou deveria fazê-lo & 1 & 2 & 3 & 4 & 5 \\
\hline Porque eu valorize pessoalmente este tipo de comportamento e é & 1 & 2 & 3 & 4 & 5 \\
\hline
\end{tabular}

\section{Por favor responda às seguintes questões:}

Quando recebe uma factura de electricidade não consegue dizer quanto gastou ou quanto custa a electricidade

Acha que a sua ultima factura reflecte precisamente o seu consumo de
Discordo

\section{Nem concordo}

nem discordo

Discordo

Nem concordo

Concordo 
E. Com que frequência efectua os seguintes comportamentos de poupança de energia quando está em casa? Por favor seleccione Não aplicável (N/A) se não possuir algum item da lista ou não se aplicar à si próprio.

\begin{tabular}{|c|c|c|c|c|c|c|}
\hline & N/A & Raramente & Ocasionalm & Algumas & Frequentem & Quase \\
\hline & & ou nunca & ente & vezes & ente & sempre ou \\
\hline & & & & & & sempre \\
\hline Usar o modo de suspender ou hibernar no computador & 0 & 1 & 2 & 3 & 4 & 5 \\
\hline Desligar as luzes quando saio de um quarto & 0 & 1 & 2 & 3 & 4 & 5 \\
\hline Comprar lâmpadas económicas/eficiência energética & 0 & 1 & 2 & 3 & 4 & 5 \\
\hline Comprar produtos orgânicos ou de produtores locais & 0 & 1 & 2 & 3 & 4 & 5 \\
\hline Usar sacos recicláveis no supermercado & 0 & 1 & 2 & 3 & 4 & 5 \\
\hline $\begin{array}{l}\text { Seleccionar transporte terrestre quando faço compras } \\
\text { online }\end{array}$ & 0 & 1 & 2 & 3 & 4 & 5 \\
\hline
\end{tabular}




\begin{tabular}{|c|c|c|c|c|c|c|}
\hline Lavar grandes quantidades de roupa (tambor cheio) & 0 & 1 & 2 & 3 & 4 & 5 \\
\hline Secar a roupa utilizando o secador & 0 & 1 & 2 & 3 & 4 & 5 \\
\hline Tomar duches curtos ( 5 minutos ou menos) & 0 & 1 & 2 & 3 & 4 & 5 \\
\hline Reduzir o consumo de carne & 0 & 1 & 2 & 3 & 4 & 5 \\
\hline Subir as escadas em vez de utilizar o elevador & 0 & 1 & 2 & 3 & 4 & 5 \\
\hline
\end{tabular}

F. As pessoas reduzem o seu consumo de energia por diversas razões. Por favor indique o grau de importância que as seguintes razões têm para si na redução do consumo de energia.

\begin{tabular}{|c|c|c|c|c|}
\hline & De alguma & & & \\
\hline Não é & forma & & Muito & Extremamente \\
\hline importante & importante & Importante & importante & importante \\
\hline
\end{tabular}

Preocupação pelo estado actual do ambiente

Redução de custos para um empregador ou para o local

onde vive

1

2

3

5

Hábito 
Preocupação pelo bem-estar das gerações futuras

Poupança pessoal/Redução de custos pessoais

Estatuto pessoal junto de amigos, colegas ou familiares
1

1

1
2

2

2
3

\section{Aparelhos que consomem energia eléctrica...}

Conhecer a família e os aparelhos que utiliza fornece informação sobre hábitos de consumo energético efectuado pela mesma. Para tal pedimos que preencha o seguinte quadro com informação sobre os aparelhos que tem em casa. Se possui algum aparelho que consome energia eléctrica e que não esteja na lista por favor acrescente-o à mesma

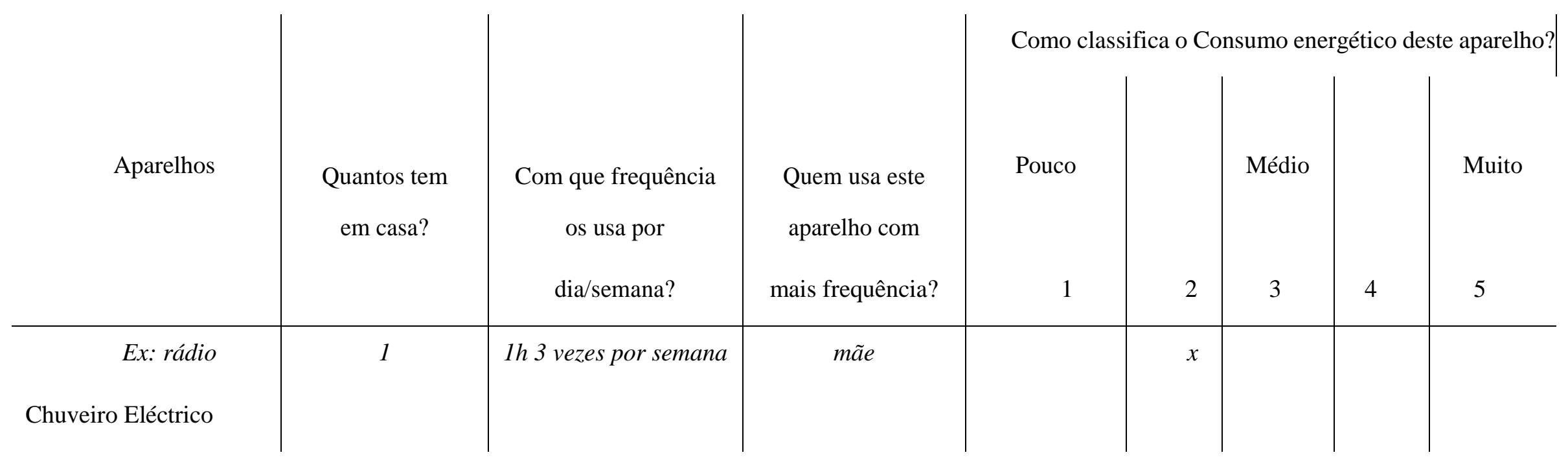


Computador(portátil/fixo

)

Máquina de lavar

Máquina de lavar loiça

Máquina de secar

Fogão Eléctrico

Forno eléctrico

Arca congeladora

Frigorífico

Tostadeira/torradeira

Chaleira eléctrica

Aparelhagem de som

Televisão 


\subsection{WattsBurning interview protocol}

\section{Concerns about energy consumption}

1. Who pays the electricity/water bills? How do you do this (services used)?

2. How much do you spend every month?

3. Do you keep a record of how much you spend? (Days, months, years)

4. Do you have any concerns about your energy consumption?

5. Do you talk about these concerns with your children/spouse/partner?

6. What kind of strategies do you take to address these concerns?

7. Ask families to describe a week/regular day of their week. And do the same for the weekend.

\section{Contextual inquiry with the power meter}

1. Can you show me how you use the system?

a) How would you check your energy consumption?

b) Who would use it more often? How often did that happened?

c) When would you check the system? Can you give a particular example?

d) Who would use it less? Why do you think that happened? Can you think of a particular reason of why that happened?

e) Was it easy for you to interpret this information?

f) What kind of information was more relevant for you? Why was that?

g) Was this information helpful for you/your daily routine?

Mobile vs fixed

h) How did the tablet change the way you see your energy?

i)Can you compare with the other system? (To those that experience the other system)

\section{Emotional engagement}

j) What do you like in this visualization? 
k) Can you identify positive aspects of the system? How about negative aspects of the system?

1) What emotions or emotional connection feelings does the visualization arise?

i) How did you feel when you were using the system?

m) What did you learn with the system?

2. Could you find a relationship between your energy consumption and the landscape shown?

3. Did you notice any changes in the landscape between the times you would check the system?

\section{Problems (strategies to solve them):}

1. Can you identify problems you had with the system?

2. How often did that happen? What did you do? How did that particular problem made you feel?

\section{Impact in the family:}

1. Did you notice any changes in your household routines?

2. Did you have to make any changes after having it at home?

3. Did you notice changes in your energy consumption?

a. Was that a concern for you?

i. Why was that?

4. Are you adopting some strategies to try to reduce your consumption?

5. Would you add anything to the system as it is right now?

a. What would you suggest that could be added to the energy meter?

b. What do you think it could be improved?

i. (How would you design this energy meter?) 
Appendices

\subsection{WattsBurning in my mailbox interview protocol}

\section{Concerns about energy consumption}

Did you change anything in your routine this week? (to perform better)

\section{The magnets}

Did you understood how the different magnets represented the consumption?

Who would place the magnets in their place? When did this happened?

Was it easy to perform the task (getting the message and placing the magnet)?

Did you had to change something in your routine to place the magnets?

Did you double check the consumption in the application.

\section{Public display}

How did you feel about having the information displayed outside?

Did you checked your neighbors consumption?

Would you agree in sharing your consumption information, in another way? (numerical) ( here we can check if having the consumption displayed in a abstract way, make them less concerned about sharing)

Did you tried to perform better than your neighbors?

Did you talked with your neighbors about this?

\section{The application}

Did you used the application more often this week?

\section{Problems?}




\subsection{What-a-Watt pre interview questionnaire}

Membro da famíla / Nome / Apartamento:

Por favor indique até que ponto sabia qual era o seu consumo de energia ao longo do dia

Não sabia

Sabia exactamente

$$
\begin{array}{lllllll}
1 & 2 & 3 & 4 & 5 & 6 & 7
\end{array}
$$

O medidor de electricidade (tablet) fez com que reduzisse o consumo?

Sim reduzi muito

$$
\text { Não reduzi nada }
$$

$$
\begin{array}{lllllll}
1 & 2 & 3 & 4 & 5 & 6 & 7
\end{array}
$$

Tomou algumas medidas ou acções para modificar os seus níveis de consumo de energia?

Não fiz nada Efectuei uma série de acções

$$
\begin{array}{lllllll}
1 & 2 & 3 & 4 & 5 & 6 & 7
\end{array}
$$

Se sim, poderia indicar que tipo de acções efectuou

Das seguintes fontes de energia, quais achas que são usada na produção de electricidade na Madeira:

\begin{tabular}{|c|l|l|}
\hline Vento & & \\
\hline Biomassa & & \\
\hline Hidro-eléctrica & & \\
\hline Carvão & & \\
\hline Sol & & \\
\hline Térmica & & \\
\hline
\end{tabular}




\begin{tabular}{|c|l|l|}
\hline Residuos Sólidos & & \\
\hline Ondas & & \\
\hline Geotérmica & & \\
\hline $\begin{array}{l}\text { Energia térmica } \\
\text { dos oceanos }\end{array}$ & & \\
\hline Gás Natural & & \\
\hline Nuclear & & \\
\hline
\end{tabular}

Ordene as técnicas que seleccionou acima em termos de:

Quota do total de energia produzida na Madeira. (escreva as 3 primeiras da mais alta para a mais baixa)

1.

2.

3.

Custo de produção na Madeira. (escreva as 3 primeiras da mais alta para a mais baixa)

4.

5.

6.

Emissões de $\mathrm{CO} 2$ durante a produção. (escreva as 3 primeiras da mais alta para a mais baixa)

1.

2.

3.

Pode identificar quais as 3 fontes de energia mais consumidas na sua casa até agora?

1.

2. 
3.

Preocupo-me com a origem da energia consumida na minha casa

Concordo totalmente Não concordo

$$
\begin{array}{lllllll}
1 & 2 & 3 & 4 & 5 & 6 & 7
\end{array}
$$

Altero/Alterei a minha rotina de acordo com a origem(s) de electricidade disponíveis no momento Sempre Nunca

$$
\begin{array}{lllllll}
1 & 2 & 3 & 4 & 5 & 6 & 7
\end{array}
$$

A hora do dia influencia que tipo de energia é produzida na ilha

Concordo totalmente Não concordo

$$
\begin{array}{lllllll}
1 & 2 & 3 & 4 & 5 & 6 & 7
\end{array}
$$

As condições metrológicas influenciam a produção de energia na ilha

Concordo totalmente Não concordo

$$
\begin{array}{lllllll}
1 & 2 & 3 & 4 & 5 & 6 & 7
\end{array}
$$




\subsection{What-a-Watt interview protocol}

\section{Concerns about energy consumption}

1. Who pays the electricity/water bills? How do you do this (services used)?

2. How much do you spend every month?

3. Do you keep a record of how much you spend? (Days, months, years)

4. Do you have any concerns about your energy consumption?

5. Do you talk about these concerns with your children/spouse/partner?

6. What kind of strategies do you take to address these concerns?

7. Ask families to describe a week/regular day of their week. And do the same for the weekend.

\section{Contextual inquiry with the power meter}

1. Can you show me how you use the system?

a) How would you check your energy consumption?

b) Who would use it more often? How often did that happened?

c) When would you check the system? Can you give a particular example?

d) Who would use it less? Why do you think that happened? Can you think of a particular reason of why that happened?

e) Was it easy for you to interpret this information?

f)What kind of information was more relevant for you? Why was that?

g) Was this information helpful for you/your daily routine?

Mobile vs fixed

h) How did the tablet change the way you see your energy?

i)Can you compare with the other system? (To those that experience the other system)

\section{Energy production}

1. What do you think about the different sources of the electricity in the island?

2. Why do you worry/don't worry/average about the source of your energy consumption?

a) Is it because of cost? Pollution?

b) Depending on the answer of the questionnaire 
c) What do you think about the cost of the energy coming from different sources?

d) Have you noticed in any pattern related to the energy production?

i.) Where you able to predict it?

e) Did you change something in your routine to maximize the usage of any source of energy. Can you specify?

\section{Problems (strategies to solve them):}

1. Can you identify problems you had with the system?

2. How often did that happen? What did you do? How did that particular problem made you feel?

\section{Impact in the family:}

1. Did you notice any changes in your household routines?

2. Did you have to make any changes after having it at home?

3. Did you notice changes in your energy consumption?

a.Was that a concern for you?

i. Why was that?

4. Are you adopting some strategies to try to reduce your consumption?

5. Would you add anything to the system as it is right now?

a.What would you suggest that could be added to the energy meter?

b. What do you think it could be improved?

i. (How would you design this energy meter?) 


\subsection{What-I-See pre study questionnaire}

Questionário sobre consumo de recursos.

Em baixo serão apresentadas afirmações sobre a relação entre os humanos e o meio ambiente. Para cada uma, por favor, indique se Concorda totalmente, Concorda Moderadamente, Não tem a certeza, ou Discorda Moderadamente ou Discorda totalmente

2. Estamos próximo do número limite de pessoas que a terra consegue suportar. * Mark only one oval per row.
Discordo
Discordo
Não tenho
Concordo
Concordo
Totalmente
Moderadamente
a certeza
Moderadamente
Totalmente

3. Os humanos têm o direito de mudar o meio ambiente para melhor satisfazer as suas necessidades.
Discordo
Discordo
Não tenho
Concordo
Concordo
Totalmente
Moderadamente
a certeza
Moderadamente
Totalmente

4. Os humanos estão a abusar do meio ambiente de uma forma severa.
Discordo
Discordo
Não tenho
Concordo
Concordo
Totalmente
Moderadamente
a certeza
Moderadamente
Totalmente

5. A intrevenção humana na natureza tem, por vezes, consequências desastrosas.
Discordo
Discordo
Não tenho
Concordo
Concordo
Totalmente
Moderadamente
a certeza
Moderadamente
Totalmente

6. O planeta Terra tem muitos recursos se aprendermos como os desenvolver.
Discordo
Discordo
Não tenho
Concordo
Concordo
Totalmente
Moderadamente
a certeza
Moderadamente
Totalmente

7. As plantas e os animais têm o mesmo direito de existir do que os humanos.
Discordo
Discordo
Não tenho
Concordo
Concordo
Totalmente
Moderadamente
a certeza
Moderadamente
Totalmente

8. O equilibrio da natureza é forte o suficiente para lidar com os impactos das nações mais modernas e industrializadas. *
Discordo
Discordo
Não tenho
Concordo
Concordo
Totalmente
Moderadamente
a certeza
Moderadamente
Totalmente

9. Apesar das nossas capacidades especiais, os humanos ainda estão sujeitos às leis da natureza.
Discordo
Discordo
Não tenho
Concordo
Concordo
Totalmente
Moderadamente
a certeza
Moderadamente
Totalmente 
10. A chamada crise ecológica em que a humanidade se encontra é muito exagerada.
Discordo
Discordo
Não tenho
Concordo
Concordo
Totalmente
Moderadamente
a certeza
Moderadamente
Totalmente

11. O planeta Terra é como uma nave espacial com espaço e recursos limitados.
Discordo
Discordo
Não tenho
Concordo
Concordo
Totalmente
Moderadamente
a certeza
Moderadamente
Totalmente

12. Os humanos foram feitos para governar sobre o resto da natureza. *
Discordo
Discordo
Não tenho
Concordo
Concordo
Totalmente
Moderadamente
a certeza
Moderadamente
Totalmente

13. O equilibrio da natureza é muito delicado e fácil de perturbar.

$\begin{array}{lllll}\text { Discordo } & \text { Discordo } & \text { Não tenho } & \text { Concordo } & \text { Concordo } \\ \text { Totalmente } & \text { Moderadamente } & \text { a certeza } & \text { Moderadamente } & \text { Totalmente }\end{array}$

14. Eventualmente os humanos irão aprender o suficiente sobre a natureza para a poder controlar.
Discordo
Discordo
Não tenho
Concordo
Concordo
Totalmente
Moderadamente
a certeza
Moderadamente
Totalmente

15. Se as coisas continuarem no rumo atual, iremos brevemente sofrer uma grande catástrofe ambiental.

$\begin{array}{lllll}\text { Discordo } & \text { Discordo } & \text { Não tenho } & \text { Concordo } & \text { Concordo } \\ \text { Totalmente } & \text { Moderadamente } & \text { a certeza } & \text { Moderadamente } & \text { Totalmente }\end{array}$

Um técnica de produção de electricidade é um método utilizado por um fornecedor de electricidade para produzir a energia que será disponibilizada para os consumidores

Em baixo serão apresentadas afirmações sobre diferentes técnicas de produção de energia e o seu impacto ambiental.

Para cada, por favor, indique se Concorda totalmente, Concorda Moderadamente,

Não tem a certeza, ou Discorda Moderadamente ou Discorda totalmente

16. Existem diferentes técnicas de produção de energia. *
Discordo
Discordo
Não tenho
Concordo
Concordo
Totalmente
Moderadamente
a certeza
Moderadamente
Totalmente

17. Diferentes técnicas de produção de energia, têm impactos ambientais diferentes.
Discordo
Discordo
Não tenho
Concordo
Concordo
Totalmente
Moderadamente
a certeza
Moderadamente
Totalmente 


\section{Appendices}

18. Preocupome com o custo ambiental da energia que consumo.

$\begin{array}{lllll}\text { Discordo } & \text { Discordo } & \text { Não tenho } & \text { Concordo } & \text { Concordo } \\ \text { Totalmente } & \text { Moderadamente } & \text { a certeza } & \text { Moderadamente } & \text { Totalmente }\end{array}$

19. Estou consciente de onde vem a energia que consumo.

$\begin{array}{lllll}\text { Discordo } & \text { Discordo } & \text { Não tenho } & \text { Concordo } & \text { Concordo } \\ \text { Totalmente } & \text { Moderadamente } & \text { a certeza } & \text { Moderadamente } & \text { Totalmente }\end{array}$

20. Sinto-me responsável pela poluição causada pela energia que consumo.

$\begin{array}{lllll}\text { Discordo } & \text { Discordo } & \text { Não tenho } & \text { Concordo } & \text { Concordo } \\ \text { Totalmente } & \text { Moderadamente } & \text { a certeza } & \text { Moderadamente } & \text { Totalmente }\end{array}$

21. Preocupome por termos de comprar energia a países estrangeiros.

$\begin{array}{lllll}\text { Discordo } & \text { Discordo } & \text { Não tenho } & \text { Concordo } & \text { Concordo } \\ \text { Totalmente } & \text { Moderadamente } & \text { a certeza } & \text { Moderadamente } & \text { Totalmente }\end{array}$

Para as seguintes afirmações por favor seleccione as 2 conclusões que expliquem de melhor forma a sua relação com o consumo de energia

22. Eu iria conter o meu consumo de electricidade *

se a electricidade fosse mais cara

se a electricidade tivesse origem num grande poluidor (grandes emissões de $\mathrm{CO} 2$ )

se conseguisse ver os maus efeitos do aquecimento global

se fosse pressionado pelos meus amigos

para mostrar aos outros que me preocupo com o meio ambiente

para me sentir melhor comigo mesmo(a)

23. Eu NÃO conteria os meus consumos de energia, *

se a electricidade fosse mais barata

se a origem da electricidade fosse renovável (baixas emissões de $\mathrm{CO} 2$ )

se fosse rico.

se fosse pressionao pelos meus amigos

para mostrar aos outros que posso pagar um conta de electricidade alta se tivesse de reduzir o conforto no meu dia a dia

Produção de energia na Madeira *

24. Por favor seleccione quais das técnicas de produção de energia acha que são utilizadas na Madeira

Vento

Biomassa

Hidroeléctrica

Carvão

Sol

Petróleo

Residuos Sólidos 
Ondas

Nuclear

Geotérmica

Energia térmica dos oceanos

Gás Natural

25. Ordene as técnicas que selecionou acima em termos de:

Quota do total de energia produzida na Madeira. (escreva as 4 primeiras da mais alta para a mais baixa)

26. Custo de produção na Madeira. (escreva as 4 primeiras da mais alta para a mais baixa)

27. Emissões de $\mathrm{CO} 2$ durante a produção. (escreva as 4 primeiras da mais alta para a mais baixa)

28. Sua idade:

29. Escolaridade

30. sexo:

Masculino

Feminino 
Appendices

\title{
8.9 What-I-See interview protocol
}

\author{
Nome: \\ Idade: \\ Produção energétrica
}

[à chegada e após uma curta apresentação, pedir para preencher o questionário NEP de forma a categorizar a demografía de utilizadores]

[depois do questionário NEP]

Prestou atenção ás condições meteorológiacas [tempo] de hoje?

Pode, por favor, as descrever [em detalhe]

[levar o utilizador até à instalação (antes da interacção)]

Sumarizar o projecto, introduzindo a base da produção energética para a RAM, i.e., quais a fontes de energia, como são gerados, o que as influência. Explicar também a representação (em percentagem) dos remoinhos e como os seus tamanhos representam quotas diferentes. Explicar também o objectivo da tomada electrica e como a sua cor representa a disponibilidade de energia renovável.

[iniciar o passo da microgeração e respondendo ao curto questionário na interface da bicicleta]

[após o passo da microgeração e da visualização da produção energética em tempo real para a RAM]

Experava/imaginou/adivinhou estes valores (quotas) para a produção energética em temporeal para a região?

Quais são as fontes de energia que se relacionam mais de perto com o que tinha pensado? Porquê?

(Qual foi)/(Quais foram) as fontes energéticas que lhe surpreendeu mais? Porquê?

[deixar o utilizador explorar e interagir com a instalação, utilizando os cartões para construir dias baseadas em valores históricos controlando variaveis meteriológicos ]

[após interagir com Watt-I-See] 
Houve alguma condição [das condições que construíu com os cartões] que fosse mais memorável/interessante? Porquê?

Consegue se relembrar das quotas (valores)? Por favor descreva-as.

Acha que consegue estimar a disponibilidade de energia renovável? Baseando-se em quê?

Ao saber das diferentes fontes, quotas de produção e as influências do tempo, muda alguma coisa na sua rotina de hoje? Porquê?

[comparar ao questionário da microgeração]

Pedalava para carregar o seu telemóvel? Porquê?

Quão dificil é?

Quanto tempo demorava?

Conseguiria pedalar para ligar a chaleira? Porquê?

Interacção com Watt-I-See (based on Kuniavsky, M)

Se o Watt-I-See estivesse disponivel para você utilizar agora, utilizaria? Porquê?

[se sim] como o utilizava/integrava nas suas rotinas diárias?

Há outra forma de que gostaria utilizar uma funcionalidade destas?

e por exemplo telemóveis?

como um ecrã em casa?

onde o locaria?

se possivel, alteraria alguma coisa [fisicamente] para esse espaço? Tais como?

Se pudesse ter somente uma funcionalidade [de Watt-I-See] o que seria? Porquê?

Se há alguma, qual foi a funcionalidade de que gostou/ajudou/preferiu menos? Porquê?

Se há alguma, qual foi a funcionalidade de que gostou/ajudou/preferiu mais? Porquê?

Se há alguma, qual foi a funcionalidade chave que faltou? Porquê? 
Appendices

Como compara Watt-I-See, com uma representação gráfica com a mesma informação e dados em, por exemplo, num computador?

Quais são as vantagens?

Quais são as desvantagens?

O Watt-I-See faz alguma coisa que é particularmente util para si? se sim, o que é?

O que o faz util?

Se houver alguma coisa, o que gosta do Watt-I-See? 


\subsection{What-I-See post study questionnaire}

Nome:

Idade:

Produção energética

[à chegada e após uma curta apresentação, pedir para preencher o questionário NEP de forma a categorizar a demografia de utilizadores]

\section{[depois do questionário NEP]}

Prestou atenção ás condições meteorológiacas [tempo] de hoje?

Pode, por favor, as descrever [em detalhe]

[levar o utilizador até à instalação (antes da interacção)]

Sumarizar o projecto, introduzindo a base da produção energética para a RAM, i.e., quais a fontes de energia, como são gerados, o que as influência. Explicar também a representação (em percentagem) dos remoinhos e como os seus tamanhos representam quotas diferentes. Explicar também o objectivo da tomada electrica e como a sua cor representa a disponibilidade de energia renovável.

[iniciar o passo da microgeração e respondendo ao curto questionário na interface da bicicleta]

[após o passo da microgeração e da visualização da produção energética em tempo real para a RAM]

Experava/imaginou/adivinhou estes valores (quotas) para a produção energética em temporeal para a região?

Quais são as fontes de energia que se relacionam mais de perto com o que tinha pensado? Porquê?

(Qual foi)/(Quais foram) as fontes energéticas que lhe surpreendeu mais? Porquê?

[deixar o utilizador explorar e interagir com a instalação, utilizando os cartões para construir dias baseadas em valores históricos controlando variaveis meteriológicos ]

[após interagir com Watt-I-See] 


\section{Appendices}

Houve alguma condição [das condições que construíu com os cartões] que fosse mais memorável/interessante? Porquê?

Consegue se relembrar das quotas (valores)? Por favor descreva-as.

Acha que consegue estimar a disponibilidade de energia renovável? Baseando-se em quê?

Ao saber das diferentes fontes, quotas de produção e as influências do tempo, muda alguma coisa na sua rotina de hoje? Porquê?

[comparar ao questionário da microgeração]

Pedalava para carregar o seu telemóvel? Porquê?

Quão dificil é?

Quanto tempo demorava?

Conseguiria pedalar para ligar a chaleira? Porquê?

Interacção com Watt-I-See (based on Kuniavsky, M)

Se o Watt-I-See estivesse disponivel para você utilizar agora, utilizaria? Porquê?

[se sim] como o utilizava/integrava nas suas rotinas diárias?

Há outra forma de que gostaria utilizar uma funcionalidade destas?

e por exemplo telemóveis?

como um ecrã em casa?

onde o locaria?

se possivel, alteraria alguma coisa [fisicamente] para esse espaço? Tais como?

Se pudesse ter somente uma funcionalidade [de Watt-I-See] o que seria? Porquê?

Se há alguma, qual foi a funcionalidade de que gostou/ajudou/preferiu menos? Porquê?

Se há alguma, qual foi a funcionalidade de que gostou/ajudou/preferiu mais? Porquê?

Se há alguma, qual foi a funcionalidade chave que faltou? Porquê? 
Como compara Watt-I-See, com uma representação gráfica com a mesma informação e dados em, por exemplo, num computador?

Quais são as vantagens?

Quais são as desvantagens?

O Watt-I-See faz alguma coisa que é particularmente util para si? se sim, o que é?

O que o faz util?

Se houver alguma coisa, o que gosta do Watt-I-See? 


\subsection{1-I-See EV2 questionnaire}

\section{VAMOS FALAR DE ENERGIA!}

1. Faz uma cruz nas energias que tu achas que são produzidas na ilha da Madeira.

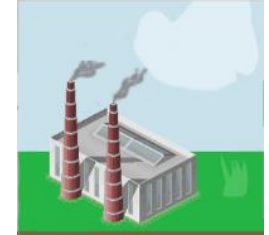

ENERGIA TÉRMICA

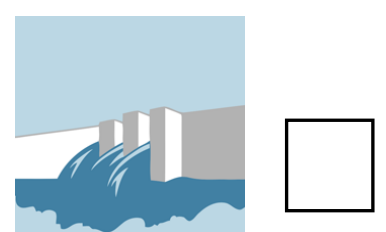

ENERGIA HIDRÁULICA

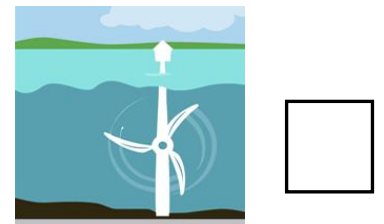

ENERGIA DAS ONDAS

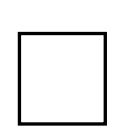

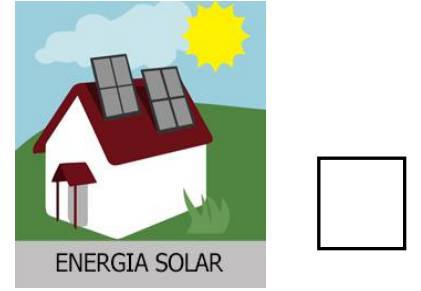
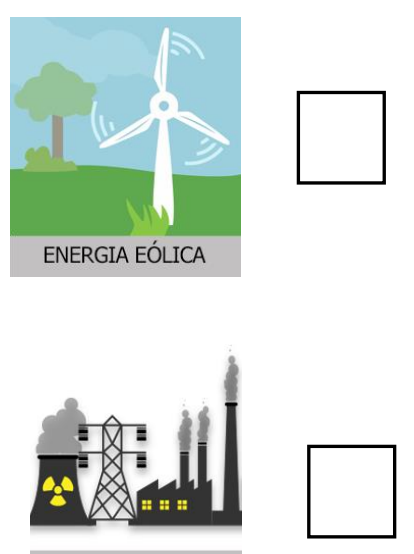

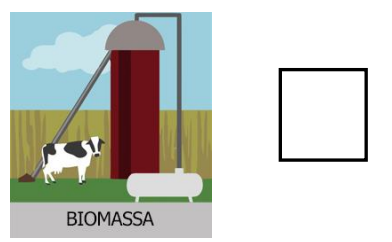

BIOMASSA

IDADE: ANOS

2. Faz uma cruz nas fontes de energia renováveis.
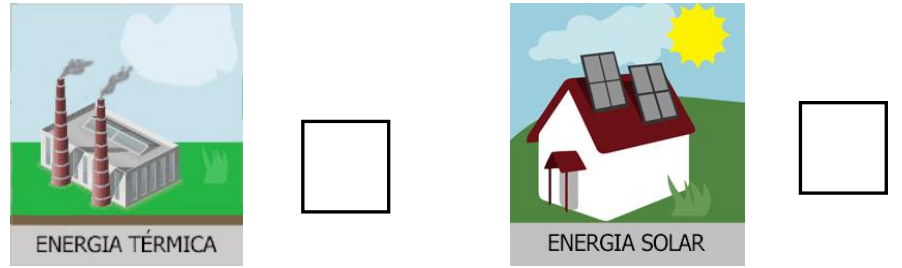

ENERGIA TÉRMICA
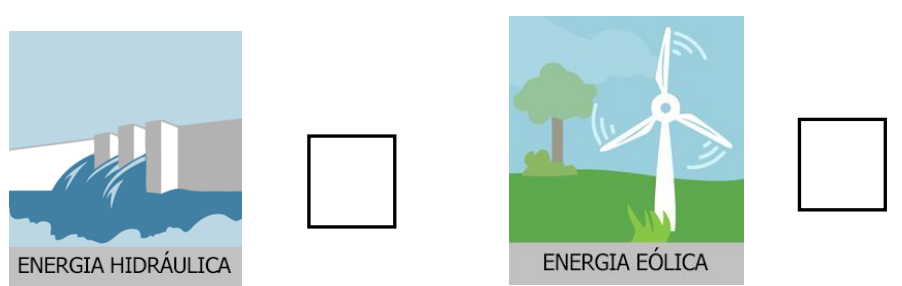

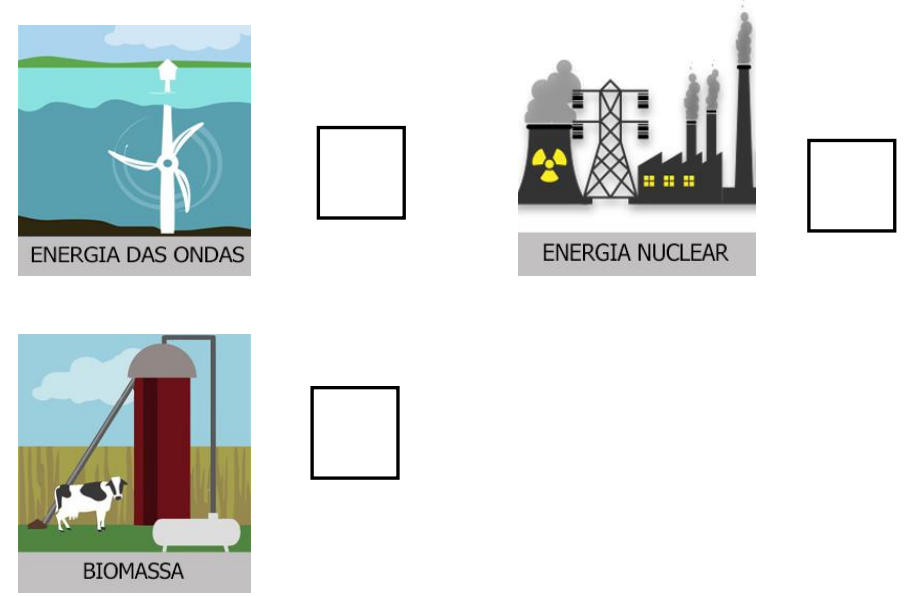

ENERGIA NUCLEAR 
Appendices

3. Faz corresponder as fontes de energia que são amigas do planeta, e as que não são.
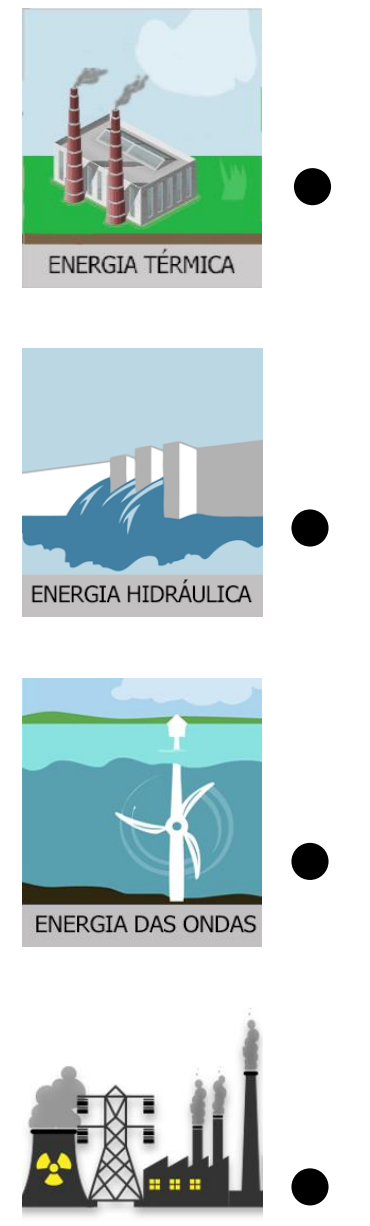

ENERGIA NUCLEAR
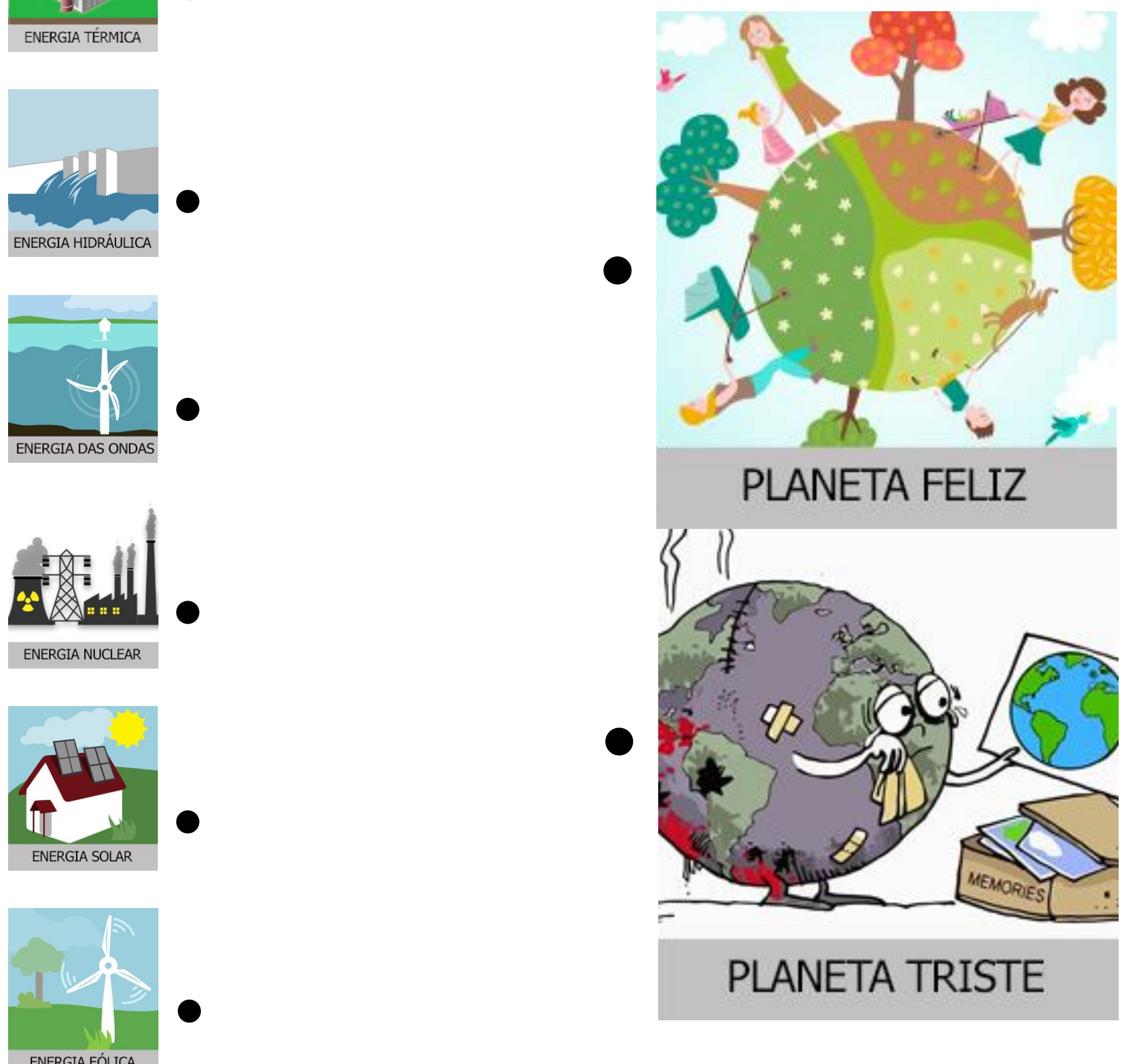

PLANETA TRISTE

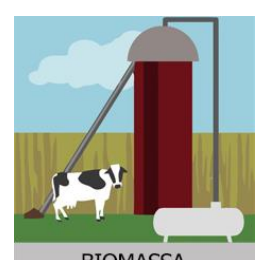

BIOMASSA 
4. Faz corresponder quais as energias mais produzidas quando está...
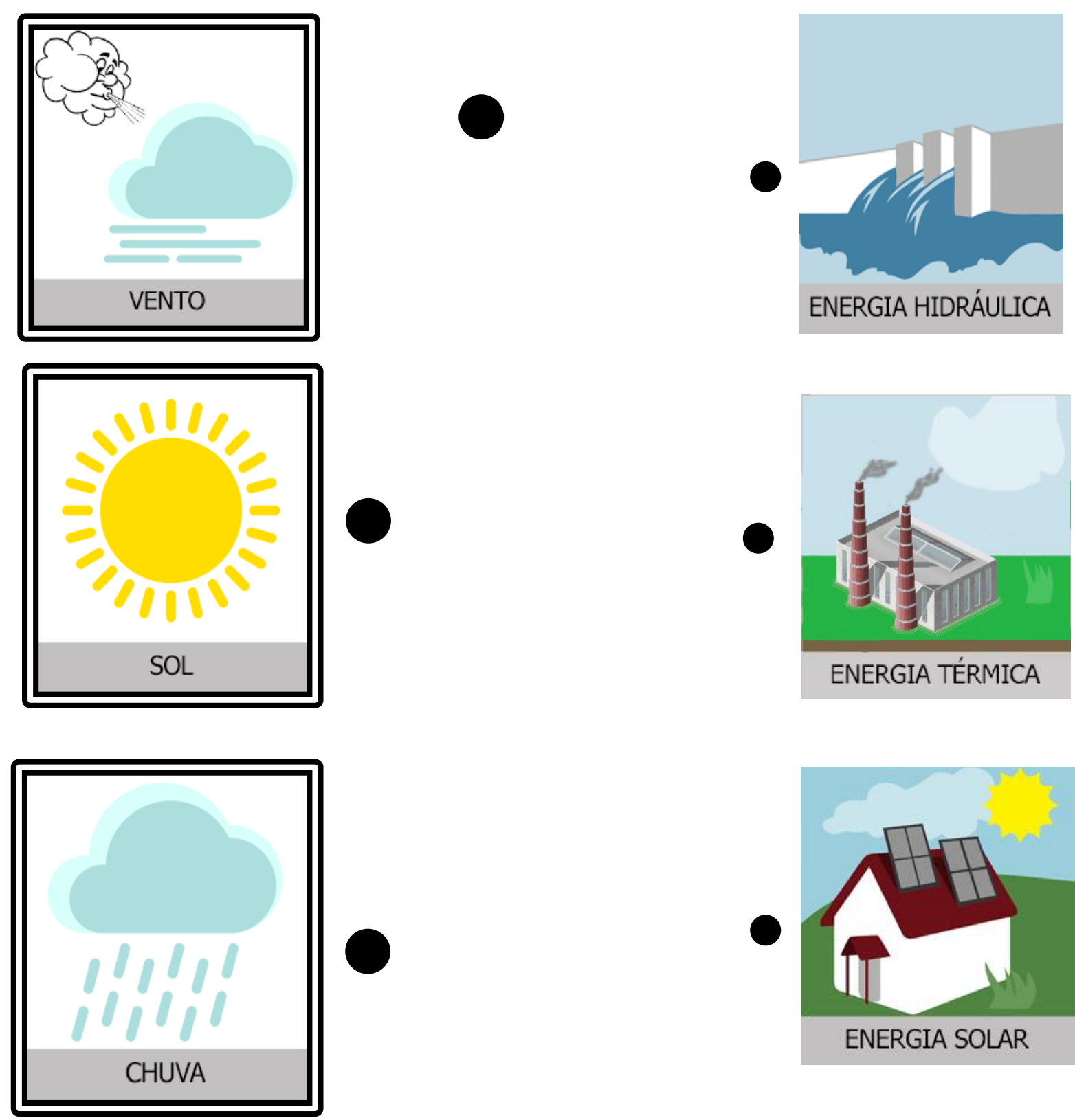
Appendices

5. Faz corresponder as energias que estão a ser produzidas nas

diferentes partes do dia.
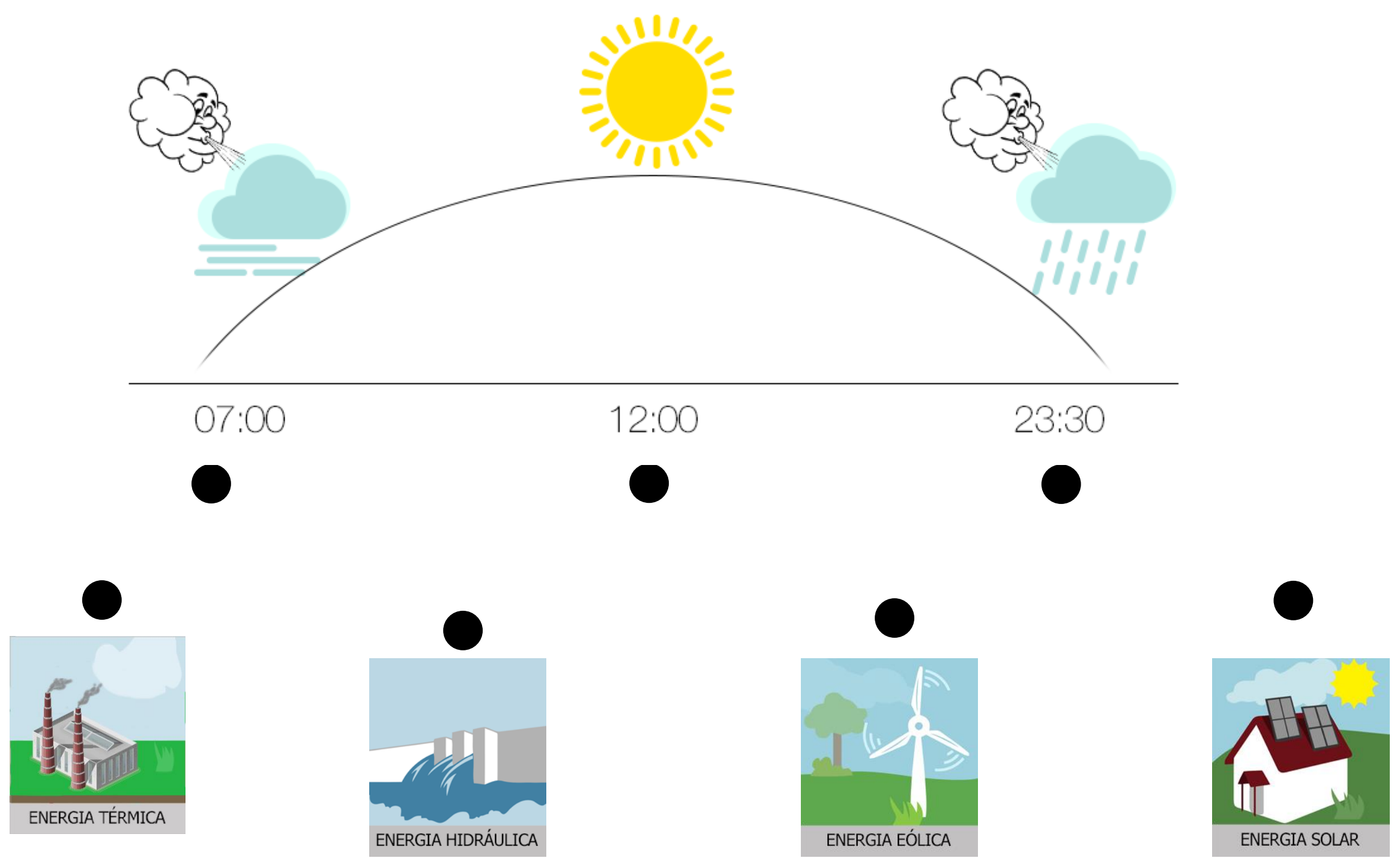
Appendices 